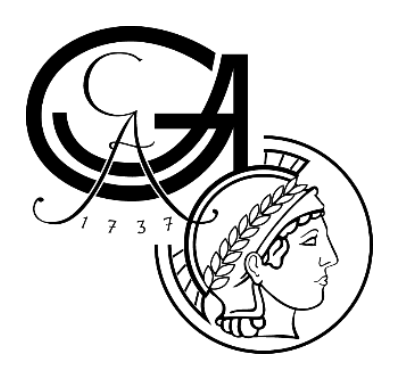

\title{
Optogenetic stimulation of the cochlea
}

\author{
Dissertation
}

\author{
for the award of the degree \\ "Doctor rerum naturalium" \\ of the Georg-August-Universität Göttingen
}

within the doctoral program "Molecular Biology"

of the Georg-August University School of Science (GAUSS)

submitted by

David López de la Morena

born in

Cuenca, Spain

Göttingen, 2018 



\section{Members of the Thesis Advisory Committee:}

\section{Prof. Dr. Tobias Moser}

Institute for Auditory Neuroscience \& InnerEarLab, University Medical Center Göttingen

\section{Prof. Dr. Silvio O. Rizzoli}

Institute for Neuro- and Sensory Physiology, University Medical Center Göttingen

Prof. Dr. Tim Gollisch

Sensory Processing in the Retina Group, University Medical Center Göttingen

Further members of the Examination Board:

\section{Dr. Nicola Strenzke}

Auditory Systems Physiology Group, University Medical Center Göttingen

\section{Dr. Manuela Schmidt}

Somatosensory Signaling and Systems Biology Group, Max-Planck-Institute of Experimental

Medicine, Göttingen

\section{Dr. Camin Dean}

Trans-synaptic Signaling Group, European Neuroscience Institute Göttingen

Date of oral examination: 18.12.2018 



\section{Declaration}

I hereby declare that this thesis has been written independently and with no other sources and aids than quoted.

David López de la Morena

Göttingen, 30. October, 2018 



\section{Contents}

\section{List of abbreviations}

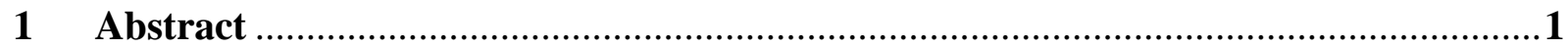

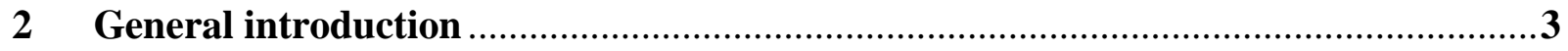

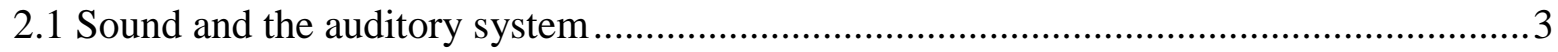

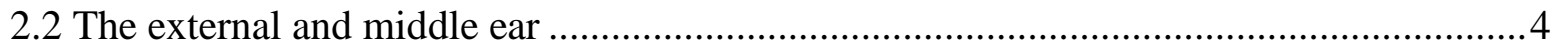

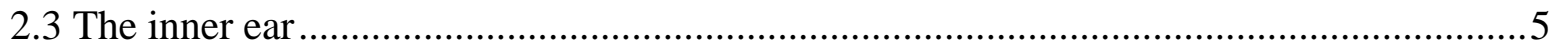

2.4 The organ of Corti and ribbon synapses ..............................................................

2.5 Innervation patterns in the organ of Corti and spike generation in SGNs .........................

2.6 Studying cochlear function through SGN responses.................................................. 11

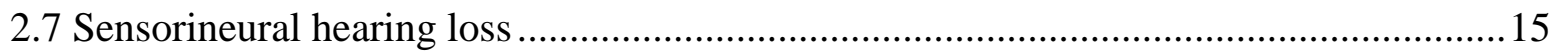

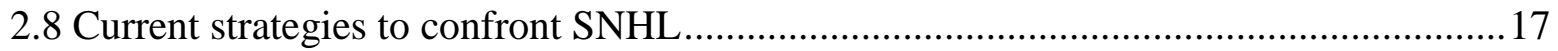

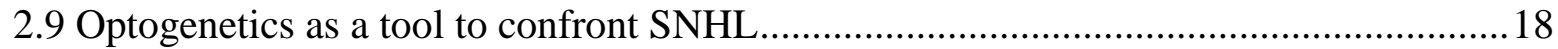

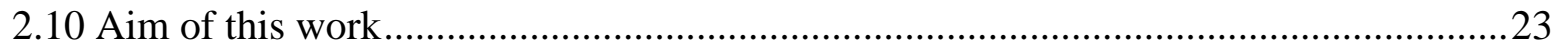

3 Ultrafast optogenetic stimulation of the auditory pathway by targeting-optimized

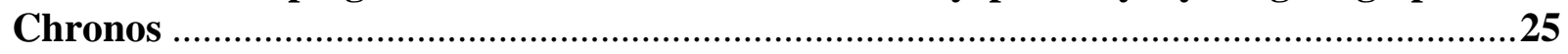

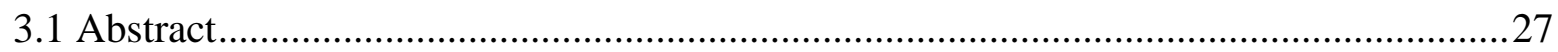

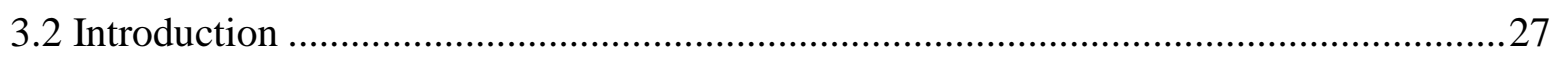

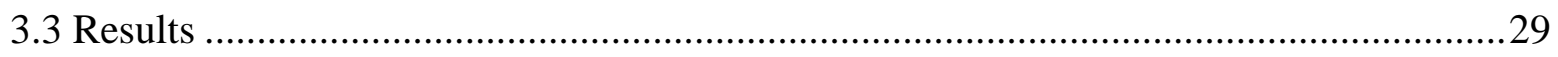

3.3.1 Chronos undergoes sub-millisecond on/off transitions at $36^{\circ} \mathrm{C}$.............................29

3.3.2 Improving the plasma membrane expression of Chronos .......................................2.

3.3.3 AAV-mediated expression of Chronos and Chronos-ES/TS in mouse SGNs ..........31

3.3.4 Chronos-mediated optogenetic neural stimulation of the auditory pathway:

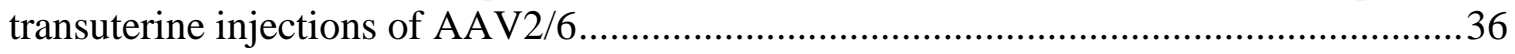

3.3.5 Chronos-ES/TS enables ultrafast optogenetic stimulation of the auditory pathway: oABRs

3.3.6 Chronos-ES/TS enables ultrafast optogenetic stimulation of the auditory pathway: recordings from single putative SGNs..........................................................................

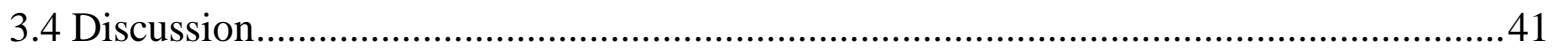

3.4.1 Biophysical characterization of Chronos and improving plasma membrane

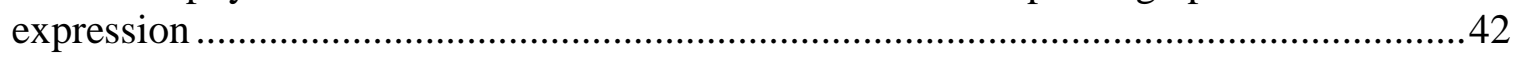

3.4.2 Ultrafast Chronos-mediated stimulation of the auditory pathway............................43

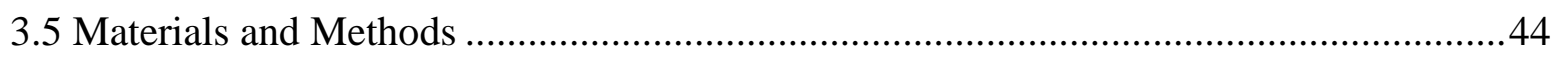

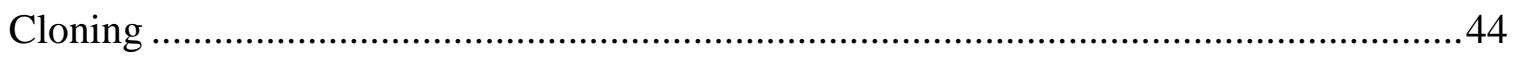




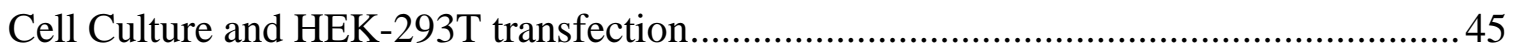

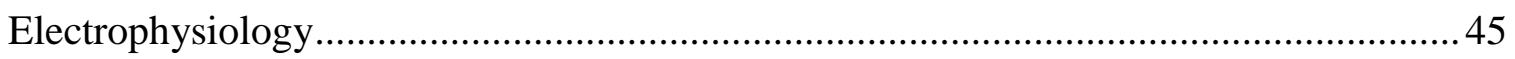

Dissociation and culture of hippocampal neurons, AAV infection and

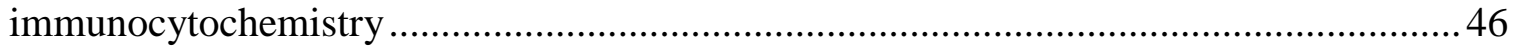

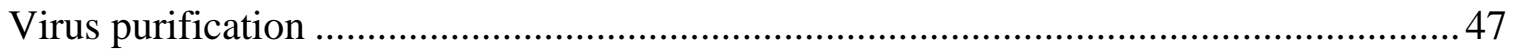

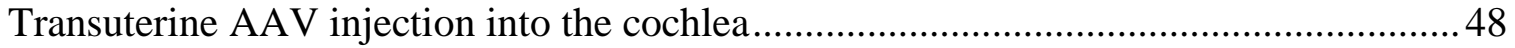

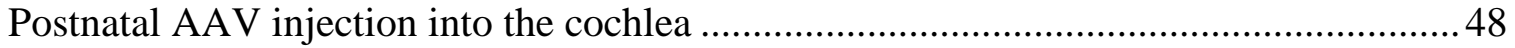

Immunostaining and imaging of cochlear cryosections .......................................... 49

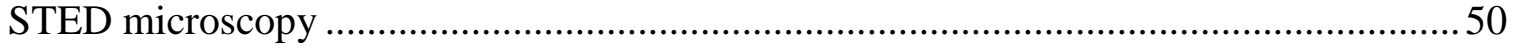

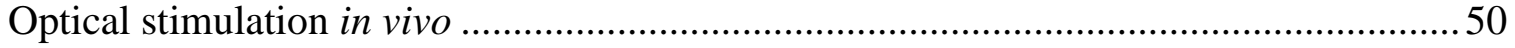

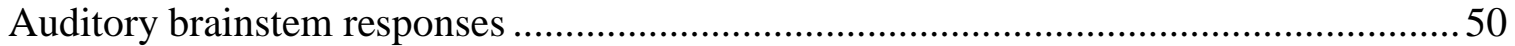

Juxtacellular recordings from single putative SGNs ..............................................51

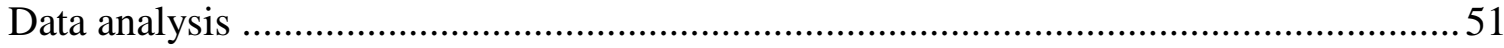

4 High frequency neural spiking and auditory signaling by ultrafast red-shifted

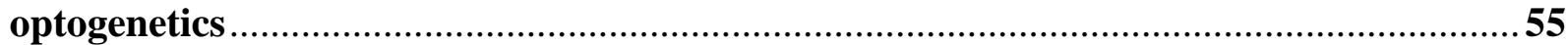

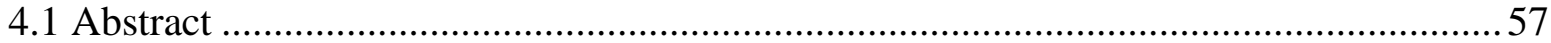

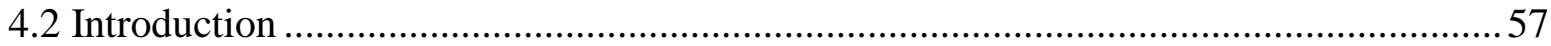

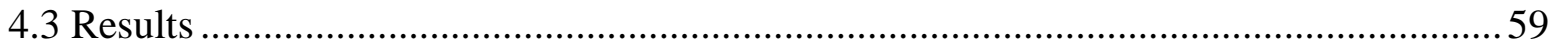

4.3.1 Fast helix F mutants and their calcium permeabilities ......................................59

4.3.2 Chrimson mutants with accelerated closing kinetics ........................................ 61

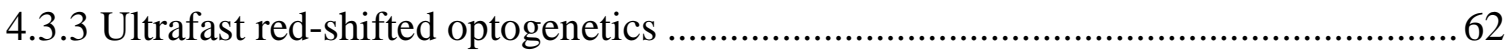

4.3.4 $\mathrm{f}$-Chrimson is a promising candidate for hearing restoration.................................65

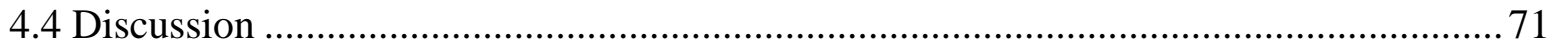

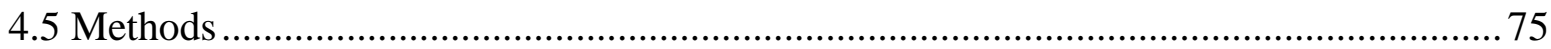

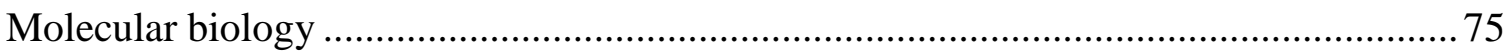

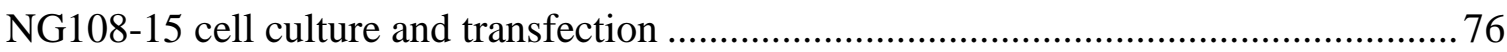

Expression of Chrimson variants in Xenopus laevis oocytes ....................................... 76

Electrophysiological recordings on Xenopus laevis oocytes.......................................76

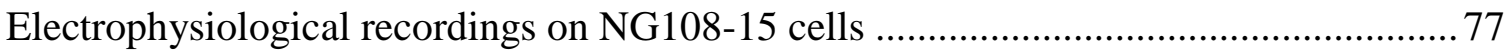

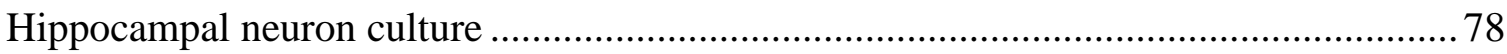

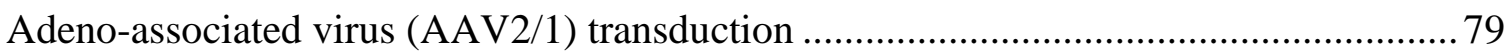

Electrophysiological recordings on hippocampal neurons........................................ 79

Animals for recordings on parvalbumin-positive interneurons .................................... 80

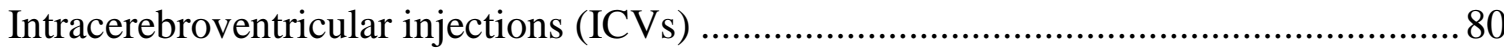

Patch-clamp recordings on parvalbumin-positive interneurons ................................... 80

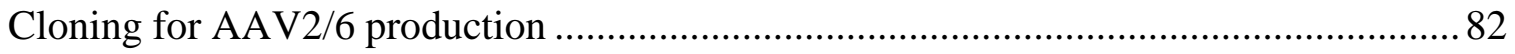




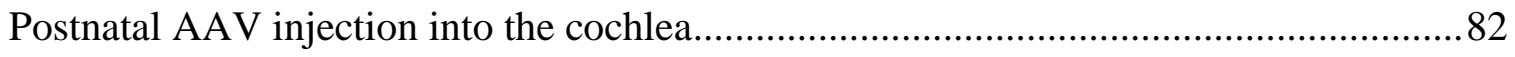

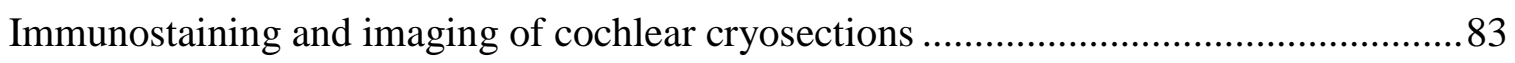

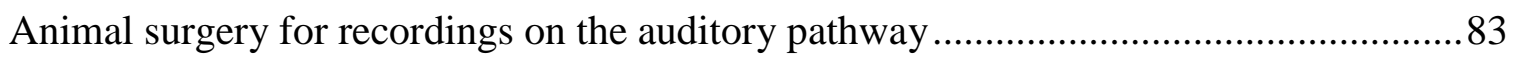

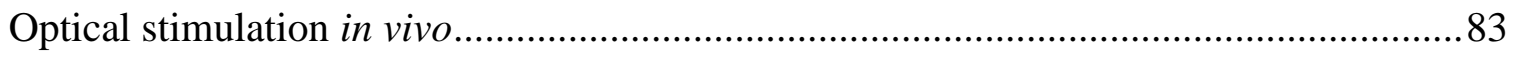

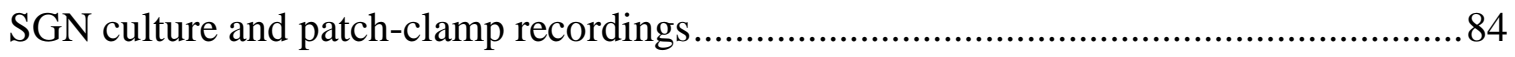

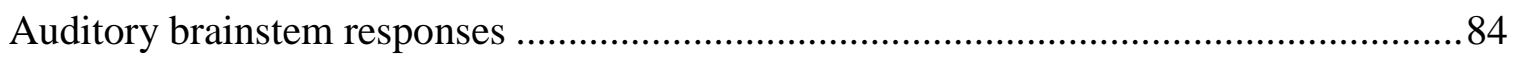

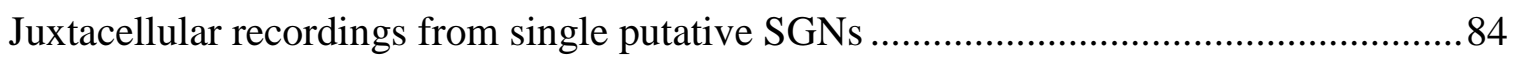

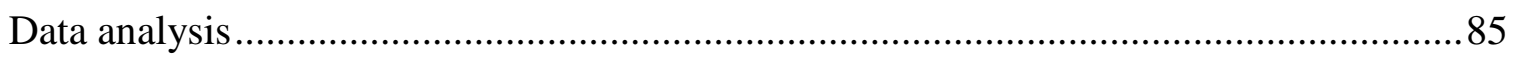

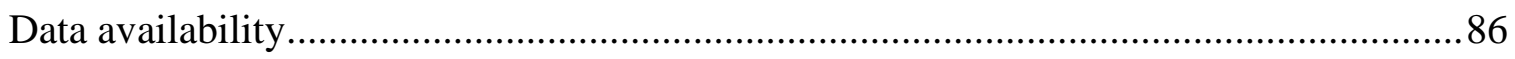

5 Characterization of optogenetic responses to varying light intensity in f-Chrimson-

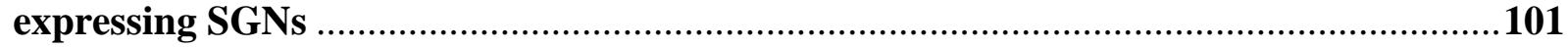

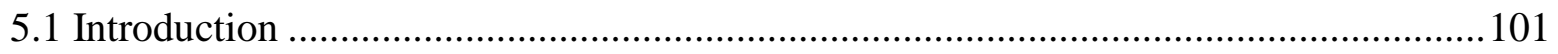

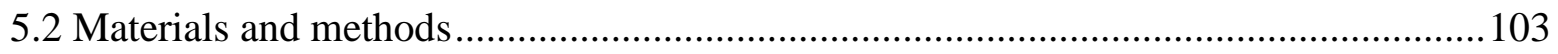

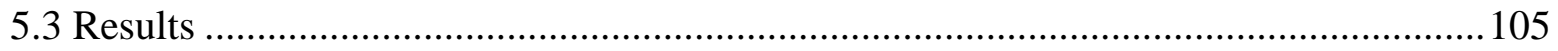

5.3.1 Analysis of f-Chrimson-EYFP expression and opsin functionality ...................... 105

5.3.2 Optogenetic spike fidelity decays with stimulation rate.................................... 106

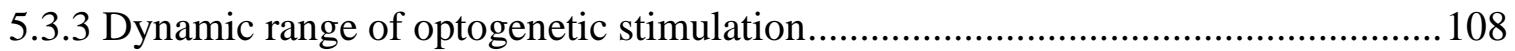

5.3.4 Temporal aspects of spiking in response to varying stimulus intensities...............110

5.3.5 Optogenetic responses to low light intensities are more reliable at the beginning of

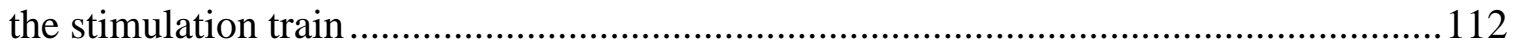

5.3.6 SGN responses to optogenetic stimulation of varying pulse rate and intensity ..... 113

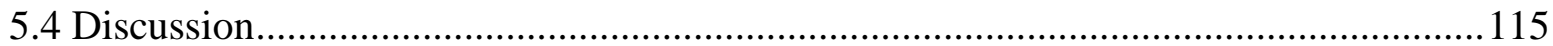

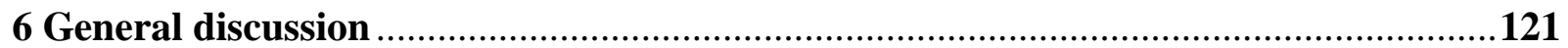

6.1 Chronos and f-Chrimson as potential candidates for clinical cochlear optogenetics .... 121

6.2 Comparison to other strategies aimed at confronting SNHL ................................... 126

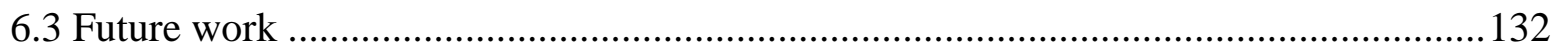

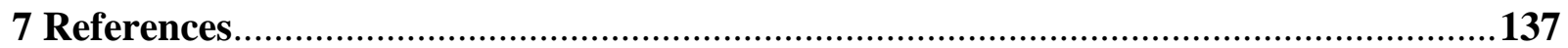

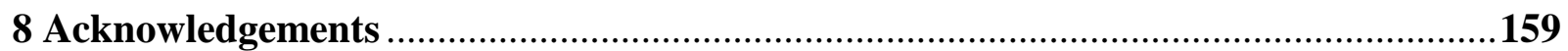

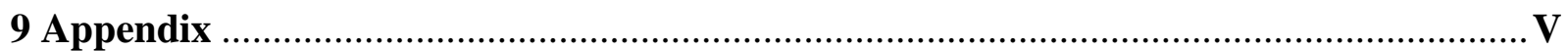




\section{List of abbreviations}

$\begin{array}{ll}\text { HLED } & \text { Micro light-emitting diode } \\ \text { AAV } & \text { Adeno-associated virus } \\ \text { ABR } & \text { Auditory brainstem response } \\ \text { aABR } & \text { Acoustic auditory brainstem response } \\ \text { AC } & \text { Alternating current } \\ \text { ACE } & \text { Advanced combinational encoder } \\ \text { AMPA } & \alpha \text {-amino-3-hydroxy-5-methyl-4-isoxazolepropionic acid } \\ \text { AP } & \text { Action potential } \\ \text { AZ } & \text { Active zone } \\ \text { CAP } & \text { Compound action potential } \\ \text { CF } & \text { Characteristic frequency } \\ \text { ChR } & \text { Channelrhodopsin } \\ \text { CI } & \text { Cochlear implant } \\ \text { CIS } & \text { Continuous interleaved sampling } \\ \text { DC } & \text { Direct current } \\ \text { DPOAE } & \text { Distortion product otoacoustic emissions } \\ \text { DR } & \text { Dynamic range } \\ \text { EAS } & \text { Electro-acoustic stimulation } \\ \text { eCI } & \text { Electrical cochlear implant } \\ \text { EYFP } & \text { Enhanced yellow fluorescent protein } \\ \text { ES } & \text { Endoplasmic reticulum-export signal } \\ \text { FSP } & \text { Fine structure processing } \\ \text { FP } & \text { Fluorescent protein } \\ \text { GFP } & \text { Green fluorescent protein } \\ \text { HC } & \text { Hair cell } \\ \text { hSyn } & \text { Human synapsin } \\ \text { IHC } & \text { Inner hair cell } \\ \text { INS } & \text { Infrared neural stimulation } \\ \text { oABR } & \text { Optical auditory brainstem response } \\ \text { oCI } & \text { Optical cochlear implant } \\ \text { OHC } & \text { Outer hair cell } \\ \text { P } & \text { Postnatal day } \\ \text { SPL (pe) } & \text { Peak equivalent sound pressure level } \\ \text { RLF } & \text { Rate-level function } \\ \text { S.d. } & \text { Standard deviation } \\ \text { S.e.m. } & \text { Standard error of the mean } \\ \text { SGN } & \text { Spiral ganglion neuron } \\ \text { SNHL } & \text { Sensorineural hearing loss } \\ \text { SPEAK } & \text { Spectral peak } \\ \text { SPL } & \text { Sound pressure level } \\ \text { SR } & \text { Spontaneous rate } \\ \text { TS } & \text { Plasma membrane-trafficking signal } \\ \text { VCSEL } & \text { Vertical-cavity surface-emitting lase } \\ \text { YFP } & \text { Yellow fluorescent protein } \\ \end{array}$




\section{Abstract}

Optogenetic stimulation of the auditory nerve is a promising alternative to restore hearing. Since light can be focused on the target tissue, the optical cochlear implant (oCI) might support a major improvement in frequency resolution compared to current electrical CIs (eCIs). However, the slow kinetics of the first generation of optogenetic actuators hindered controlling the fast spiking spiral ganglion neurons (SGNs) with high temporal fidelity.

In this work, I evaluated the ability of two fast-gating opsins (Chronos-ES/TS and f-Chrimson) to improve temporal fidelity of optogenetic stimulation of murine SGNs in vivo. Following postnatal injections of adeno-associated virus (AAV) via the round window, channelrhodopsin expression under the human synapsin promoter rendered SGNs light-sensitive without detrimental effects in neuronal survival.

Improved plasmalemmal expression of Chronos-ES/TS in SGNs ensued from the usage of the potent AAV.PHP.B capsid and the addition of endoplasmic reticulum export signals (ES) and plasma membrane trafficking signals (TS) to the Chronos transgene. High and homogeneous expression levels of Chronos-ES/TS in SGNs throughout the cochlea ( 70\%) allowed high spike probability and temporal precision of spiking up to $1 \mathrm{kHz}$ with moderate energy thresholds assessed by optically-evoked auditory brainstem responses (oABR) ( 7 $\mu \mathrm{J}$ per pulse).

On the other hand, the fast-gating opsin f-Chrimson opened up the new avenue of fast redshifted cochlear optogenetics. AAV2/6-mediated expression of f-Chrimson was high and homogeneous in the injected ear $(\sim 80 \%)$, allowing optical activation of the auditory pathway with low oABR energy thresholds (down to a minimum of $0.5 \mu \mathrm{J}$ per pulse) and restoring auditory activity at pulse rates up to $250 \mathrm{~Hz}$ in hearing-impaired mice 9 months post-injection. Further characterization at the single-neuron level revealed sub-millisecond temporal jitter of spiking up to pulse rates of $400 \mathrm{~Hz}$ and improved average dynamic range of optogenetic coding compared to electrical stimulation $(\sim 4 \mathrm{~dB}$ vs $\sim 1 \mathrm{~dB})$ that rose up to $\sim 8 \mathrm{~dB}$ at the population level.

Therefore, these preclinical results pave the way for an increased frequency and intensity resolution in cochlear prosthetics and advance cochlear optogenetics by overcoming major obstacles that had so far hampered clinical translation of the oCI: enabling physiological stimulation rates with low light intensities in postnatally transduced animals. 


\section{General introduction}

\subsection{Sound and the auditory system}

Sensation arises from the capacity of living organisms to perceive information conveyed by stimuli of different nature. Throughout evolution, mammals have developed highly specialized structures and systems that allow them to deal with the distinct sensory modalities. Despite being very diverse, sensory pathways share a few common roots: sensory processing begins when a receptor helps to transduce the energy associated with a stimulus into neural signals, and higher neural centers then interpret them. In humans, the basic senses comprise somatic sensation, balance, gustation, olfaction, audition and vision. Although the nature of humans is highly visual, the auditory system plays a major role in communication, detection of threats and enjoyment e.g. through the appreciation of music.

Sound can be physically described as a pressure wave that propagates spherically from the source and produces a periodic compression and rarefaction of an elastic medium, e.g. air. Sound waves feature four major characteristics: amplitude, frequency, phase and waveform. In terms of perception, the amplitude and frequency of a sound wave at the ear correlate to loudness and pitch, respectively. The vast majority of natural sounds comprise complex waveforms that can be represented as the sum of diverse pure sinusoidal waves of varied amplitudes, frequencies and phases through a mathematical process dubbed the Fourier transformation. Notably, an equivalent process occurs at the level of the inner ear, where sound is decomposed into its constituting frequencies.

Each mammalian species is able to perceive a different range of frequencies and intensities of sound stimuli. Regarding sound intensity, the human auditory system can encode sounds over a remarkably broad dynamic range from 0 to $120 \mathrm{~dB}$ SPL, i.e. 6 orders of magnitude (Kandel et al., 2012). Factors like functional head size show an inverse relation to the ability to perceive high frequencies (Heffner and Heffner, 2008). While the human hearing range roughly spans frequencies from $20 \mathrm{~Hz}$ to $20 \mathrm{kHz}$ (Purves et al., 2004), mice are sensitive to sounds ranging from 1 to about $100 \mathrm{kHz}$ (Ashmore, 2008). Despite this difference, the mouse has become the most commonly used mammal in auditory neuroscience, largely due to the rise of inbred strains and their advantages concerning genetic manipulation (Ohlemiller et al., 2016). 


\subsection{The external and middle ear}

The external ear of mammals consists of the pinna and the ear canal (Figure 2.1). The pinna acts as a collector, focusing airborne sound into the ear canal, where it is channeled onto the tympanic membrane. The pinna also changes the frequency spectrum, thereby providing a cue for sound source localization along the vertical axis and also prevents front-back confusions (Heffner and Heffner, 2008).
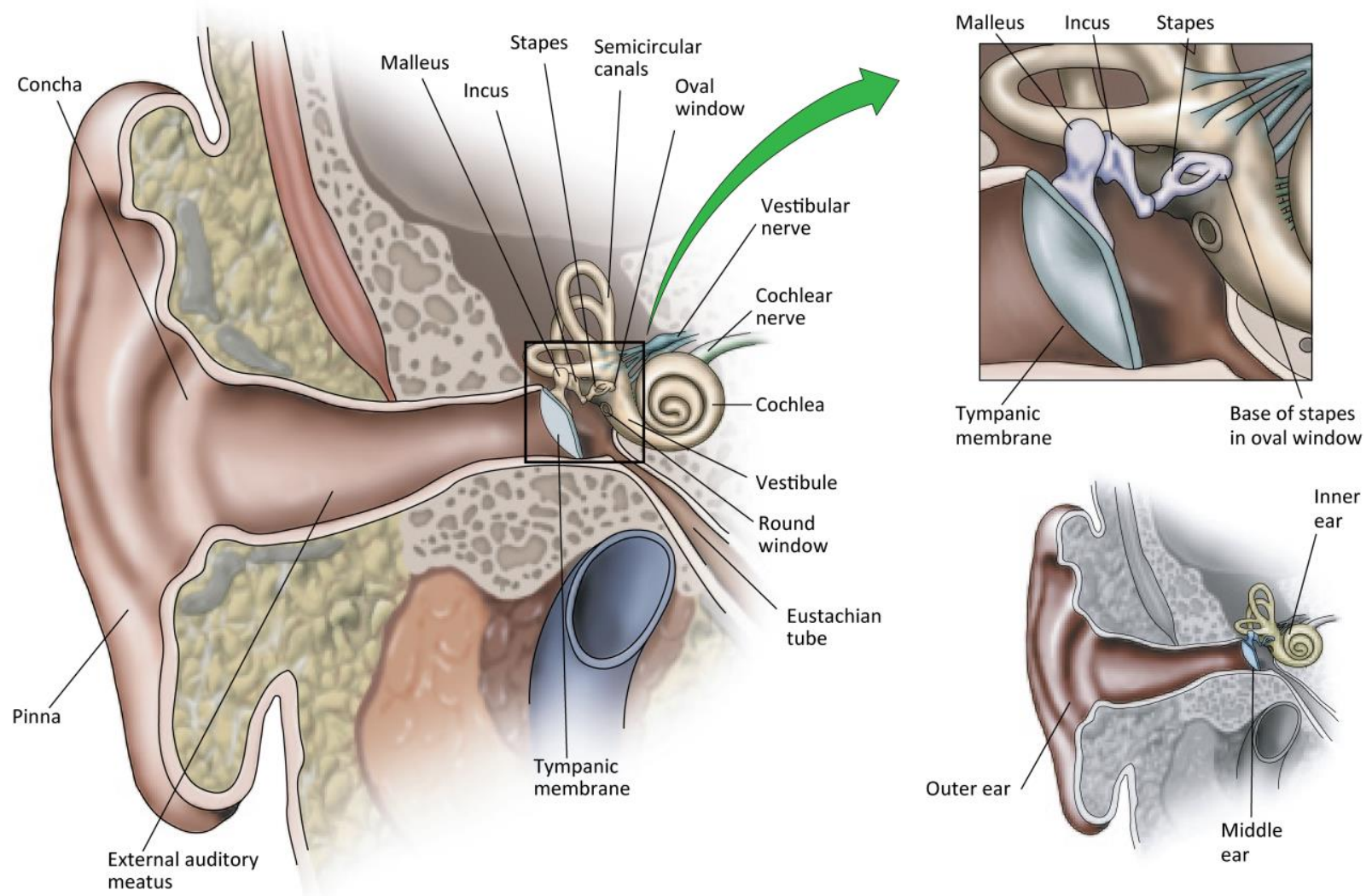

Figure 2.1: Schematic of the human ear

The human ear is divided into three main compartments: the external, middle and inner ear (lower right). The tympanic membrane is connected to the oval window via the ossicle chain of the middle ear, positioned between them. (Modified from (Purves et al., 2004)).

The tympanic membrane is the anatomical barrier that separates the external and middle ear (Figure 2.1, upper right). The middle ear further comprises the middle ear cavity and a chain of interconnected ossicles: the malleus, incus and stapes. These ossicles are attached to the tympanic membrane via the malleus and act as conveyers of air-borne sound vibrations to the fluid-filled cochlea. The main function of this structure is to match the relatively low-impedance of the air outside the ear to the much higher impedance of cochlear fluids. This process is 
essential to prevent the reflection of energy by cochlear perilymph and is achieved through the lever action of the ossicle chain and mainly through the difference in surface area between the tympanic membrane and the much smaller oval window, to which the flattened base of the stapes is connected. The result is an increase in pressure by almost 200 -fold when vibrations reach the inner ear (Purves et al., 2004).

\subsection{The inner ear}

The inner ear houses the sensory organs for balance and audition in a continuous bony shell termed the labyrinth, which is divided into three compartments. The vestibule and semicircular canals shelter the vestibular epithelia, which detect linear acceleration and rotation, respectively, contributing to the sense of balance and spatial orientation. The snail-shaped cochlea constitutes the third division of the labyrinth and harbors the auditory sensory epithelium - the organ of Corti. The cochlea is a tube-like structure wound around a conical bony core termed the modiolus. There are two basal openings in it: the oval window-covered by a membrane and the base of the stapes - and the round window - sealed solely by an elastic membrane. Inside, the cochlea is partitioned throughout by the flexible basilar membrane and the Reissner's membrane into three fluid-filled compartments: the scala vestibuli, the scala media and the scala tympani (Figure 2.2B); except for a small region at the cochlear apex - the helicotrema-where scala tympani and scala vestibuli meet. This opening allows the $\mathrm{Na}^{+} / \mathrm{Cl}^{-}$-rich perilymph that fill both scalae to mix, whereas the scala media contains the $\mathrm{K}^{+}$-rich medium endolymph (Fettiplace, 2017).

Hence, the scala vestibuli is delimited by the Reissner's membrane and extends from the oval window to the apical helicotrema, while the scala tympani runs from this point to the round window, being bordered by the basilar membrane. The scala media lies between the two and contains the organ of Corti (Figure 2.2B,C).

Thus, sound waves reach the base of the stapes and force the perilymph in scala vestibuli to be displaced. This produces a travelling wave alongside that set the basilar membrane in vertical motion, and eventually causes the round window membrane to deflect outwards (Figure 2.3A). 


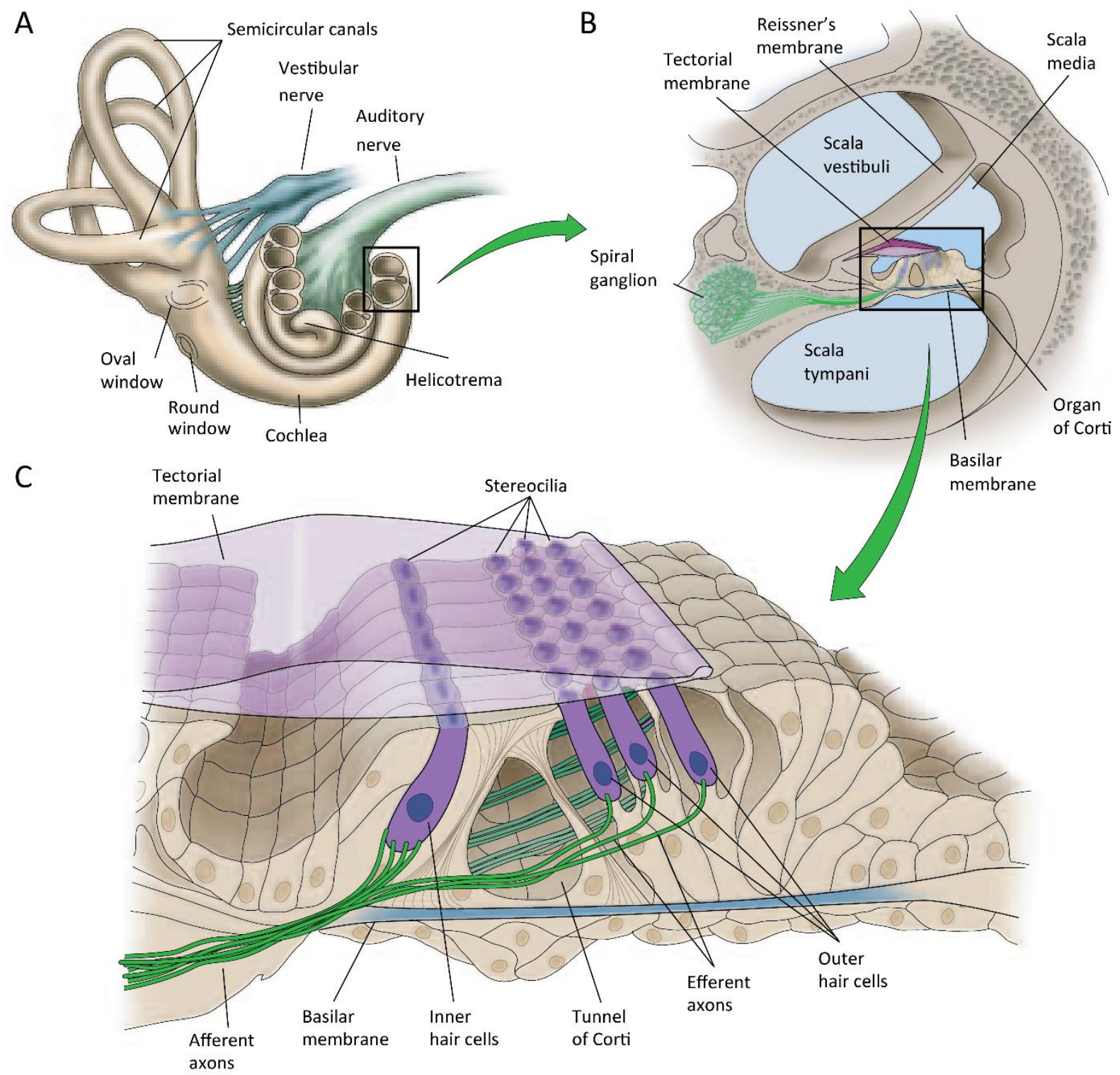

Figure 2.2: Anatomy of the cochlea and the organ of Corti

A: Schematic of the bony labyrinth depicting the cochlea with its oval and round window, the vestibular organ and both the vestibular and auditory nerve. B: Cross section of the cochlea highlighting the area marked in A, showing its three compartments and the position of the organ of Corti between scala media and scala tympani. C: Detailed anatomical organization of the organ of Corti, showing its characteristic single row of inner hair cells (IHCs) and three parallel rows of outer hair cells (OHCs), the tectorial (purple) and basilar membrane (blue) as well as afferent and efferent innervation (modified from (Purves et al., 2004)).

Due to the gradually varying mechanical properties of the basilar membrane along the cochlear axis - narrow and stiff at basal positions to wide and soft at the apex-sounds of different frequencies produce maximal motions at specific positions along the basilar membrane (Figure 2.3B). Therefore, the travelling wave originates in the stiffer end and propagates toward the 
apex with an increasing amplitude and reduction in velocity, until it reaches a spot of maximal vibration that is dependent on the sound frequency. Consequently, the basal, firmer end responds best to higher frequencies whereas the apical, more flexible region is responsive to lower frequencies. This gives rise to a topographical frequency gradient termed tonotopy, which is maintained at successive stations of the auditory pathway determining the organism's frequency hearing range (reviewed in (Fettiplace, 2017)). This feature allows the basilar membrane to passively decompose complex sound signals into their fundamental spectral components, thereby acting as a Fourier analyzer (Dallos, 1996; Mann and Kelley, 2011). However, as suggested by the non-linear properties of the basilar membrane motion (Rhode, 1971), other factors contribute to maximizing this effect. Among them, we could highlight the different properties and structural organization of the tectorial membrane depending on the tonotopic position (Gueta et al., 2006; Richter et al., 2007; Richardson et al., 2008) and the amplification provided by outer hair cells (OHC) (reviewed in (Ashmore, 2008; Dallos, 2008)). In any case, tonotopy along the cochlear spiral is a critical feature, which allows the radially arranged afferent fibers of spiral ganglion neurons (SGNs) to establish a position-based neural frequency code.
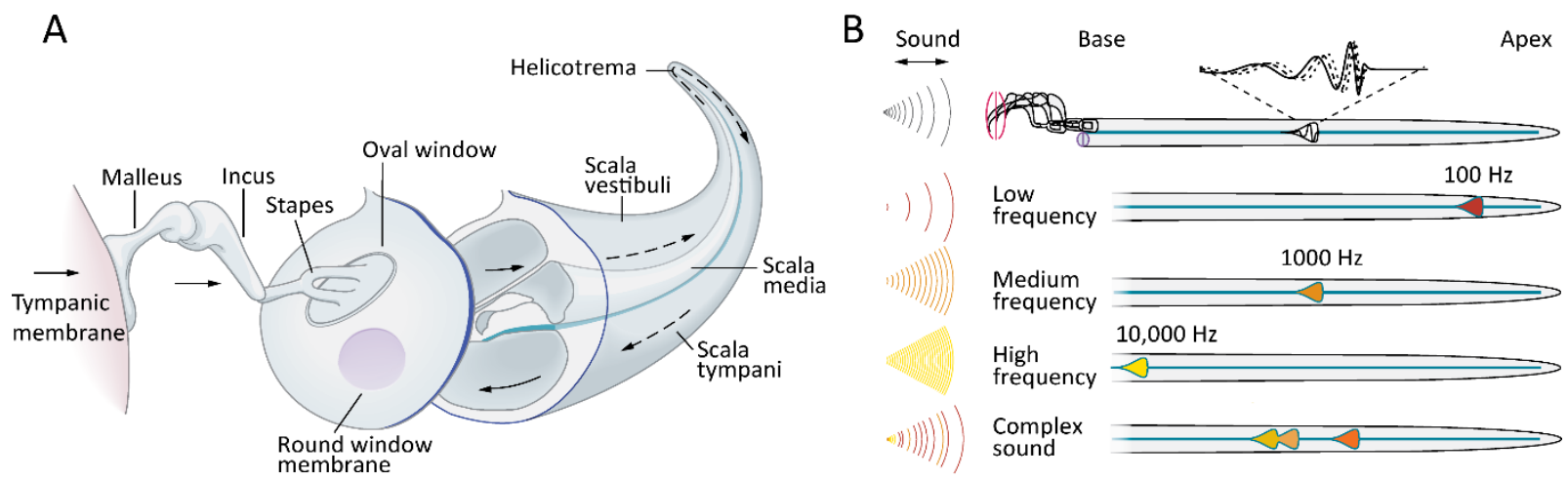

Figure 2.3: Flow of energy in the cochlea and tonotopy

A: Simplified schematic of an uncoiled cochlea showing the chain of events upon sound stimulation (arrows) B: Sound vibrations propagate along the basilar membrane as a travelling wave from the basal to the apical region (upper panel). The gradually changing mechanical properties of the basilar membrane give rise to its tonotopic distribution, meaning that maximal motion is elicited at specific positions of the basilar membrane in a frequency-dependent manner (lower panels) (modified from (Kandel et al., 2012)).

\subsection{The organ of Corti and ribbon synapses}

The organ of Corti harbors hearing receptor hair cells (HCs) and is responsible of mechanoelectrical transduction of sound. The HCs are organized in three rows of OHCs and a 
single row of inner hair cells (IHCs), which are disposed in parallel to the basilar membrane along its length. Both OHCs and IHCs develop an apical bundle of stereocilia arranged in several rows of increasing height. OHCs express the "motor" protein prestin, which confers them electromotility (Zheng et al., 2000) for their function as amplifiers of the incoming sound signal. OHCs receive only $~ 5 \%$ of afferent innervation, while the bulk of the afferent fibers reach IHCs ( 95\%, type I fibers) and identify them as the genuine hearing receptors (Fettiplace, 2017). At each tonotopic position, the vibration of the basilar membrane deflects stereocilia against the tectorial membrane, resulting in the gating of mechanoelectrical transducer channels on top of IHCs hair bundles. Thus, cation influx (mainly $\mathrm{K}^{+}$) causes graded depolarization of IHCs, which in turn opens voltage-gated $\mathrm{Ca}^{2+}$ channels at their basolateral membrane (Fettiplace and Hackney, 2006). At the active zones (AZ) of the basolateral IHC membrane, the increase in $\mathrm{Ca}^{2+}$ triggers the release of glutamate-filled synaptic vesicles into the synaptic cleft (Brandt et al., 2005). AMPA receptors at the afferent SGN postsynaptic bouton bind the released glutamate (Fuchs et al., 2003) triggering the depolarization of the SGN, which eventually fires action potentials (APs) that propagate via the VIII cranial nerve (vestibulocochlear nerve) to the cochlear nucleus (Figure 2.2A).

The first auditory synapse is characterized by its high release rates and temporal precision (Fuchs, 2005; Köppl, 1997). SGN spike rates can well be above $1 \mathrm{kHz}$ at sound onset and maintain an adapted discharge in the range of hundreds of $\mathrm{Hz}$ (reviewed in (Lagnado and Schmitz, 2015; Moser and Vogl, 2016; Wichmann and Moser, 2015)). Such exquisite ability seems to be mediated by an electron-dense structure at AZs - mainly composed of the scaffold protein RIBEYE (Magupalli et al., 2008; Schmitz, 2009) — termed the ribbon, which can also be found in photoreceptors, vestibular hair cells and bipolar cells in the retina among others (reviewed in (Matthews and Fuchs, 2010)). The molecular architecture of ribbon synapses seems to be very different than that of "conventional" neuronal synapses, apparently lacking many of the commonly found synaptic proteins (Moser and Vogl, 2016; Wichmann and Moser, 2015) and having their own specific complement instead (Pangrsic et al., 2012). Functionally, ribbon synapses deviate from conventional synapses in their ability to respond to graded potentials, i.e. neurotransmitter release is regulated by stimulus intensity. Hence, IHCs allow to encode timing information and respond over large intensity ranges, since the rate of transmitter release translates into higher spike rates (Fuchs, 2005). 
The ribbon tethers a pool of vesicles around it and thus, it is hypothesized to have a major role in replenishing vesicle release sites (Frank et al., 2010; von Gersdorff et al., 1996; Jean et al., 2018; Maxeiner et al., 2016). Other plausible ribbon functions include: coordinating vesicle release sites with $\mathrm{Ca}^{2+}$ channels at the AZ (Frank et al., 2010; Khimich et al., 2005), regulating $\mathrm{Ca}^{2+}$ channels function (Jean et al., 2018), maintaining a population of vesicles close to release sites (Becker et al., 2018), limiting $\mathrm{Ca}^{2+}$ spread at areas close to the AZ (Graydon et al., 2011) or even limiting the speed of vesicle release (Jackman et al., 2009).

\subsection{Innervation patterns in the organ of Corti and spike generation in SGNs}

The innervation pattern in the organ of Corti is very structured and comprises different types of neural fibers. SGNs are the primary sensory neurons in the auditory pathway. Morphologically, they are bipolar neurons that pack their somata inside the bony modiolus, forming the spiral ganglion in Rosenthal's canal (Figure 2.2B). Their peripheral neurites contact hair cells while their central processes run to the cochlear nucleus through the VIII cranial nerve. From there, successive synaptic relays ascend to the contralateral superior olive, the inferior colliculus, the thalamic medial geniculate body, and finally the auditory cortex. Structurally, SGNs are divided into two categories (Figure 2.4). Type I SGNs are wide, with myelinated and unbranched fibers. Their somata can be myelinated (as in the cat) or not (as in humans) (Rattay et al., 2013). Type I SGNs account for $\sim 95 \%$ of all and contact IHCs. Each AZ is contacted by a single postsynaptic SGN bouton, so that information carried by each afferent fiber arises from individual AZs (Liberman, 1980). On the other hand, 10-30 afferent fibers make synaptic contact with each IHC, and thus, several neurons encode information from each tonotopic location (Frank et al., 2009; Liberman, 1982b) with a number of contacts that correlates with auditory sensitivity (Liberman et al., 1990). The remaining 5\% of the SGNs is constituted by type II afferents. They are unmyelinated along their length, have a narrower caliber and present a ramified pattern innervating 30-60 OHCs. The density of type II fibers is not evenly distributed in the cochlea, showing a gradient that decreases from the apical to the basal region (Liberman et al., 1990). Deciphering the function of type II SGNs has remained elusive, but recent studies suggest their role in suppressing cochlear amplification (Froud et al., 2015) and in auditory nociception (Flores et al., 2015; Liu et al., 2015). 


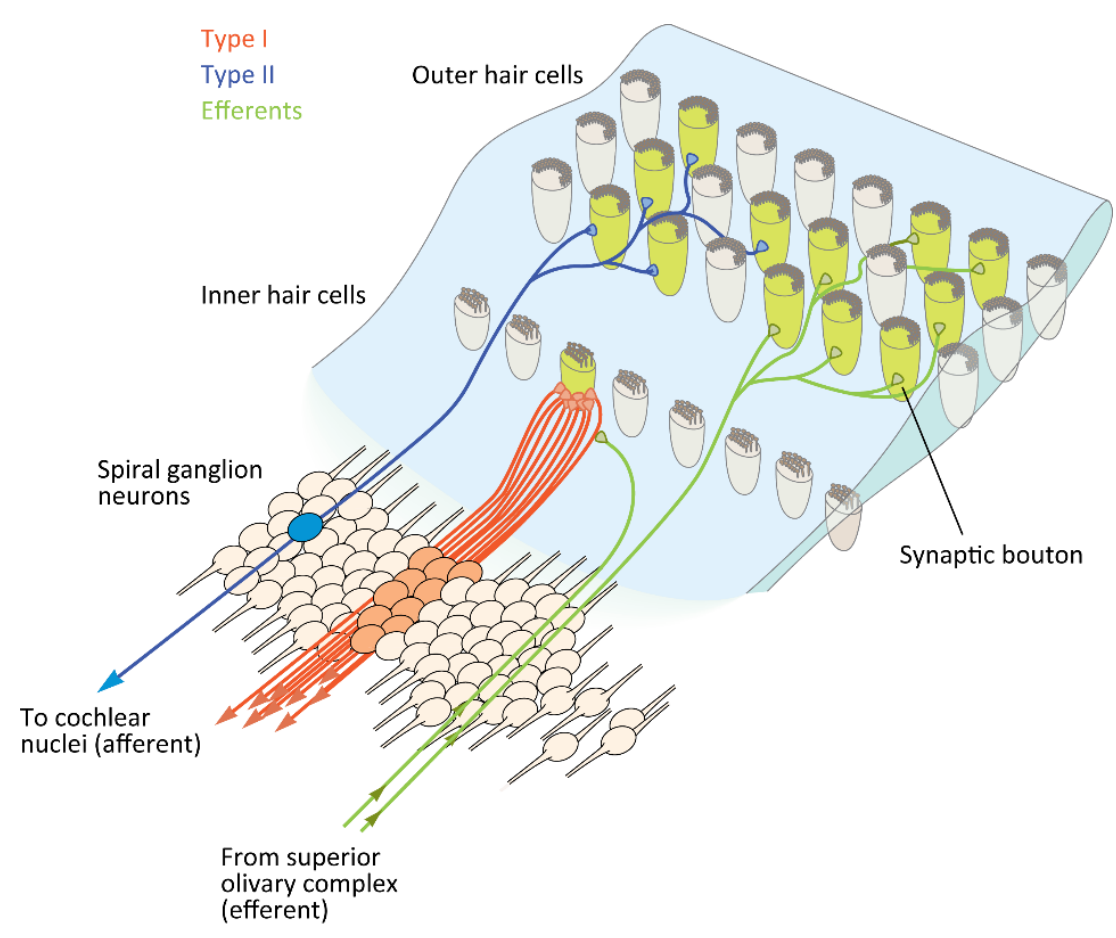

Figure 2.4: Innervation in the organ of Corti

Type I fibers (orange) transmit sensory information from individual IHCs and account for $~ 95 \%$ of the total afferent innervation in the cochlea. Type II fibers (blue) constitute the remaining $\sim 5 \%$ and show a branched pattern that innervate multiple OHCs. Most efferent axons from the superior olivary nuclei (green) directly innervate OHCs, but a number of them also regulates type I fiber activity (modified from (Kandel et al., 2012).

The organ of Corti, also receives efferent innervation from the superior olivary nuclei that provides inhibitory input to OHCs and to the postsynaptic afferent boutons of SGNs, perhaps functioning as a feed-back mechanism (Brown, 1987). Efferent myelinated fibers innervating OHCs descend from the medial superior olive, whereas unmyelinated efferents originate in the lateral superior olive (Brown, 1987; Liberman et al., 1990).

Among the diverse neuronal fibers innervating the organ of Corti, type I SGNs transmit the biggest share of the sound-coding information at high rates and with high temporal precision. Particularly, type I SGNs are unmyelinated at their postsynaptic segments just beneath the IHCs, and only after they pass through the slits of the habenulae perforata myelination starts. The first heminode-located at this point-is thought to be the first spike generator in type I fibers (Rutherford et al., 2012), likely due to its high densities of $\mathrm{Na}_{\mathrm{v}} 1.6$ channels (Hossain et al., 2005; Lysakowski et al., 2011), a common feature as well in the pre-terminal segment and successive nodes of Ranvier. High $\mathrm{Na}_{\mathrm{v}} 1.6$ densities could lower the AP threshold and reduce spike latency at recepto-neural segments, and also support transmission at nodes of Ranvier (Hossain et al., 
2005). Due to the low synaptic threshold, almost every release event in the presynapse triggers a single AP in the postsynapse, if it is not refractory from previous firing (Rutherford et al., 2012; Siegel, 1992). Further features supporting the high temporal precision of SGN spiking include their phasic excitability and the higher mean evoked EPSC amplitude ( $\sim 300$ pA) relative to their low rheobase ( $50 \mathrm{pA})$ (Rutherford et al., 2012).

\subsection{Studying cochlear function through SGN responses}

Methods such as distortion-product optoacoustic emissions (DPOAE), auditory brainstem response (ABR) and compound action potential (CAPs) measurements provide a valuable gross assessment about the general status of cochlear function. As non-invasive procedures, they enable to maintain the auditory system in a native state while the experimenter collects insightful data rather quickly. However, those experiments lack the resolution required to provide information about the basic sound encoding apparatus: the single AZ-SGN synapses.

Despite being more invasive, juxtacellular recordings from individual SGNs can pinpoint physiological aspects of hearing at the single neuron level. This way, collecting data from multiple SGNs compose a much more detailed description of sound encoding at the primary sensory neurons.

Over the years, researchers have studied SGN activity in different mammal species. Pioneering work in guinea pig (Tasaki, 1954) and in the cat (Kiang et al., 1965; Liberman, 1978) have found continuity with other animal models as the gerbil (Ohlemiller and Echteler, 1990), the mouse (Taberner and Liberman, 2005) or the chinchilla (Temchin et al., 2008). While most of the field have focused on SGN responses to sound stimuli, some groups characterized auditory nerve responses to electrical stimulation (Dynes and Delgutte, 1992; Miller et al., 1999, 2001, 2006, 2008).

The study of SGN activity show heterogeneous patterns of SGN response properties, which however, depict common features across different mammal species.

\section{Frequency tuning}

SGNs are frequency-tuned. This means that the intensity threshold level of SGN firing is lowest for sounds of a given frequency, the so-called characteristic frequency $(\mathrm{CF})$. Together, fibers of different $\mathrm{CF}$ cover the entire hearing range of the animal and define its frequency-selective sensitivity (Heil and Peterson, 2015; Meyer and Moser, 2010). This feature arises from the 
tonotopic mapping of the cochlea described above, which is maintained in the auditory nerve (Greenwood, 1996). The relationship between threshold and sound frequency describes the tuning curve for each fiber, which generally shows a prominent minimum at $\mathrm{CF}$ and higher thresholds for the rest of the audible spectrum (Figure 2.5A) (Kiang et al., 1965; Liberman, 1978; Taberner and Liberman, 2005). Low thresholds and narrow frequency tuning reflect the amplifier function of OHCs (Kiang et al., 1976).

\section{Spontaneous firing rate}

SGNs show a spontaneous, basal rate of AP firing termed the spontaneous rate (SR) that ranges from 0 to 120 spikes per second in the mouse (Taberner and Liberman, 2005). Species as the cat show a clear bimodal SR distribution across SGNs, where SR is negatively correlated with threshold (Liberman, 1978). This distribution is not as clear in the mouse, but rather continuous. However, an inverse correlation of SR and threshold is still present (Taberner and Liberman, 2005). In the cat, despite the bimodal distribution of fibers either around low or high SR, Liberman established three SGN groups based on a correlation of SR with threshold (Liberman, 1978). Thus, SR usually serve as a classifier of SGNs and it can be used to infer other SGN properties. Recently, it has been reported that high-SR fibers in gerbils use spike timing to encode sound in background noise, whereas low-SR fibers rely on rate for performing this task (Huet et al., 2018). Furthermore, in gerbils, the proportion of fibers with higher or lower SR seems dependent on CF (Bourien et al., 2014; Ohlemiller and Echteler, 1990). Differences in SR also correlate with the SGN contact location at the IHC. Fibers reaching the modiolar (neural) side of IHCs tend to have a lower SR than those projecting onto the pillar (abneural) side (Liberman, 1982a). This suggests that pre- and postsynaptic factors play a role in establishing the heterogeneity observed for SR (Heil and Peterson, 2015; Meyer and Moser, 2010). Hence, ribbons, $\mathrm{Ca}^{2+}$ channel clusters and $\mathrm{Ca}^{2+}$ current amplitude are larger at the modiolar side (Frank et al., 2009; Ohn et al., 2016) while AMPA receptor patches have been suggested to follow an opposing gradient (Liberman et al., 2011), features that are likely influenced by olivocochlear efferents (Yin et al., 2014). In turn, different SR groups among SGNs arise from the differential protein expression patterns of broadly varied functions, ranging from $\mathrm{Ca}^{2+}$-channel regulators to transcription factors. Additionally, IHC-driven activity is crucial for the development of SGN identities (Shrestha et al., 2018). Moreover, a recent study reported a more depolarized operating range of presynaptic $\mathrm{Ca}^{2+}$ channels at the modiolar side 
when compared to the pillar side (Ohn et al., 2016). This could contribute to the observed reduction in SR for fibers reaching the modiolar side, but also predict a lower sound evoked vesicle release for the same stimulus intensity (Ohn et al., 2016). Further comparison of these SGNs subtypes show that low-SR and high-SR fibers have, respectively, small and large fiber diameter and mitochondrial content (Gleich and Wilson, 1993; Liberman, 1982a; Liberman and Oliver, 1984), which could help to explain the correlation of SR with threshold (Geisler et al., 1985) but specific studies addressing this question remain to be performed (Meyer and Moser, 2010).

\section{Rate-level functions}

A third important feature in SGN responses is the correlation of spiking rate with stimulus intensity, giving rise to the rate-level function (RLF). As described above, there is a direct correlation between the SR of an SGN and its sensitivity to stimulation. Low-SR fibers require a higher stimulus intensity to increase their firing rate, but their firing rate monotonically increases for wide intensity ranges. In contrast, spiking rate in high-SR fibers reaches saturation within milder increments of stimulus strength (Ohlemiller et al., 1991; Sachs et al., 1989; Taberner and Liberman, 2005; Winter et al., 1990). This is quantified by the dynamic range (DR) of SGNs, i.e. the range of stimulus intensity for which the firing rate increases from 10 to $90 \%$ of the difference between spontaneous and maximal rates (Figure 2.5B). Thus, DR correlates directly with SGN threshold and inversely with SR (Yates et al., 1990). Therefore, each IHC steers spiking of a repertoire of SGNs with diverse response properties. Together, these correlations suggest that the intensity range discernible by an organism results from the cooperation of fibers with varied characteristics, arising from each tonotopic region (Meyer and Moser, 2010). In the mouse, most RLFs have DRs $<20 \mathrm{~dB}$ SPL in response to pure tones at CF, show hard saturation and can be approximated using sigmoid functions (Taberner and Liberman, 2005). There, narrower DRs and steeper RLFs might be related to the specialization of this animal for high frequencies. Most of the mouse hearing range falls above sound frequencies for which spike synchronization to the stimulus waveform carries significant information (see below); therefore, the rise of spiking rate with sound intensity is more pronounced than in other animals (Ohlemiller et al., 1991; Taberner and Liberman, 2005). 

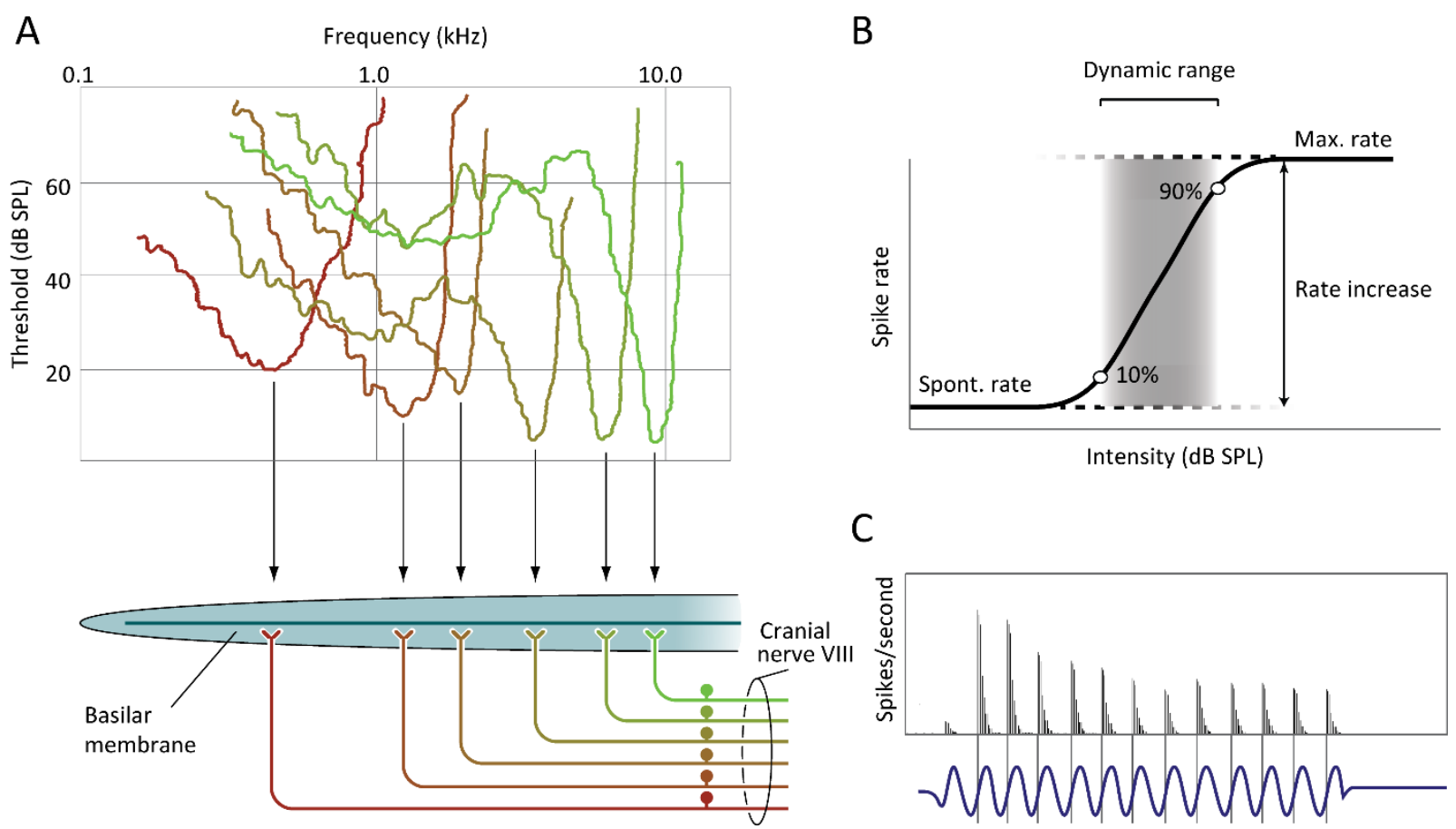

Figure 2.5: Response properties of SGNs

A: Exemplary tuning curves of several SGNs from the cat show minimum intensity thresholds for preferred sound frequencies (CF) (top panel). This feature arises from the tonotopic distribution of the basilar membrane. Approximate points of innervation along the basilar membrane are shown in the bottom panel (modified from (Purves et al., 2004), as C). B: Ratelevel functions represent SGN response rates as stimulus intensity increases. The calculation of the dynamic range of the fiber is defined by the sound intensities required to elicit $10 \%$ and $90 \%$ of the difference between spontaneous and maximal rates. C: Response histograms of a low frequency SGN to a 50 -ms pure tone $(260 \mathrm{~Hz}, 56 \mathrm{~dB}$ SPL) show prominent, phase-locked responses to a specific phase of the stimulus waveform (bottom).

\section{Phase-locking}

When SGNs are stimulated with periodic stimuli, they tend to fire APs at the same phases of the stimulus period, a phenomenon termed phase-locking (Figure 2.5C). While the notion of entrainment (the ability of a fiber to fire APs in each stimulus period (Avissar et al., 2013)) quantifies spike fidelity, phase-locking assesses the temporal precision of firing relative to the stimulus period (Heil and Peterson, 2015). Phase-locked spikes are important for sound localization (reviewed in (Grothe et al., 2010)) and for pitch recognition (Cariani and Delgutte, 1996). The measure used to evaluate the extent of phase-locking is the vector strength (or synchronization index) (Goldberg and Brown, 1969) a scalar that ranges from 0 (indicating a random relationship of APs with the stimulus period) to 1 (indicating perfect phase-locking). Usually, the Rayleigh test of circular data is used to estimate the significance of the obtained 
vector strength (Hillery and Narins, 1987). The quality of phase-locking depends on stimulus intensity and frequency (Heil and Peterson, 2015), and is inversely correlated with SR (Johnson, 1980). As sound intensity increases, vector strength grows over 20-30 dB until reaching saturation (Johnson, 1980). In terms of sound frequency, phase-locking falls off dramatically when pure tones approach $4 \mathrm{kHz}$, but variance among species exists (Heil and Peterson, 2015) (see (Temchin and Ruggero, 2010) for the chinchilla, (Versteegh et al., 2011) for the gerbil, (Taberner and Liberman, 2005) for the mouse and (Köppl, 1997) for the barn owl). The cause of this decay relates to the IHC membrane capacitance acting as a low-pass filter that attenuates the AC component of the receptor potential as the frequency increases. At sufficiently high frequencies, only the DC component remains (Palmer and Russell, 1986). For the cat, the maximum sound frequency that elicits significant phase-locking is higher than the average at $\sim 6$ $\mathrm{kHz}$, (Johnson, 1980); whereas for the barn owl this maximum increases up to $\sim 10 \mathrm{kHz}$ tones (Köppl, 1997). The mouse is specialized for high frequencies, i.e. there is a low proportion of SGNs with CFs at $<4 \mathrm{kHz}$. For these few low frequency fibers, the synchrony of the response is similar than in other animals. In contrast, for the rest of the fibers, phase-locking seems to be absent. Therefore, synchronization of SGN responses to pure tones does not seem to be particularly relevant in this animal (Taberner and Liberman, 2005). However, phase-locked spikes also occur in response to the envelope of higher frequency sounds (Dreyer and Delgutte, 2006), to amplitude-modulated tones (reviewed in (Joris et al., 2004)) and to clicks (Kiang et al., 1965; Lin and Guinan Jr, 2000; Pfeiffer and Kim, 1972).

\subsection{Sensorineural hearing loss}

Over 430 million adults and 34 million children worldwide ( $>5 \%$ of the world's population) suffer from disabling hearing loss, the most common human sensory impairment. Far from being a minor concern at present, projections estimate that these figures will double by 2050 . Hence, disabling hearing loss is a major public health issue that directly impacts communication and often results in feelings of isolation, depression and lessening of job prospects (WHO, 2018). In sensorineural hearing loss (SNHL) dysfunction originates in the inner ear and it represents the most common type of hearing impairment (Smith et al., 2005).

SNHL involves deficient transduction or transmission of signals to the brain and usually proceeds with loss of HCs or their synapses (Moser and Starr, 2016; Youm and Li, 2018; Zhang 
et al., 2018). Among the multiple underlying causes, genetic factors account for around $50 \%$ of the cases of congenital hearing loss (Smith et al., 2005; Zhang et al., 2018) while one-third of acquired SNHL cases is attributable to noise-induced damage (Duan et al., 2004). In addition, age-related hearing loss (presbycusis) is the most typical form of hearing impairment in the elderly, affecting one in three adults over age 60 (Fioretti et al., 2014).

\section{Genetic forms of SNHL}

Genetic disorders leading to SNHL target proteins of very varied function, with more than 100 loci associated with human monogenic hearing loss (Richardson et al., 2011; Wichmann, 2015). Defects downstream mechanoelectrical transduction affect, for instance, OHCs. There, mutations in the Slc26a5 gene coding for prestin lead to aberrant splicing and hamper cochlear amplification (Toth et al., 2007). In IHCs, auditory synaptopathies cause ribbon synapse dysfunction and affect critical proteins for hearing function such as otoferlin or VGluT3. Around 90 pathogenic otoferlin mutations have been described (Santarelli et al., 2015), leading to defective IHC ribbon synapse exocytosis and underlying prelingual hearing impairment (DFNB9) (Yasunaga et al., 1999) or temperature-dependent deafness (Varga et al., 2006). On the other hand, postlingual hearing loss (DFNA25) arises from abnormal glutamate release due to mutations in VGluT3 (Ruel et al., 2008; Seal et al., 2008). Additionally, disorders of the $\mathrm{Ca}^{2+}$ channel complex (channelopathies) can alter synaptic function. Mutations in the pore-forming $\mathrm{Ca}_{\mathrm{v}} 1.3 \alpha 1$ subunit (Baig et al., 2011) lead to impaired $\mathrm{Ca}^{2+}$ current (Platzer et al., 2000) and stimulus-coupled exocytosis (Brandt et al., 2003) causing syndromic hearing loss (Baig et al., 2011). Alternatively, alterations in the $\mathrm{Ca}^{2+}$-binding protein 2 (CABP2) underlie non-syndromic hearing deficits (DFNB93) (Schrauwen et al., 2012) by disturbing the inhibition of $\mathrm{Ca}_{\mathrm{v}} 1.3$ channel inactivation (Picher et al., 2017). Finally, SNHL can also arise from SGNs dysfunction. For instance, missense mutations in the OPAl gene, important for mitochondrial shaping, result in failed SGN activation leading to syndromic autosomal dominant optic atrophy (Yu-Wai-Man et al., 2010) likely due to peripheral neurite degeneration (Moser and Starr, 2016).

\section{Acquired forms of SNHL}

The most common forms of acquired SNHL are caused by exposure to loud noise and ageing. In both cases, dysfunction or loss of OHCs and IHCs is typical, being common that OHCs loss 
occurs first through ageing, according to data obtained in mice and rats (Yang et al., 2015). In contrast, noise trauma seems to primarily cause fibrocytes loss in the spiral ligament (Hirose and Liberman, 2003) and would affect HCs in a frequency-dependent manner only after prolonged exposure or higher intensities (Wang et al., 2002). In addition, loss of IHC ribbon synapses contributes to noise-induced hearing loss (Henry and Mulroy, 1995; Kujawa and Liberman, 2009) and to presbycusis (Sergeyenko et al., 2013; Stamataki et al., 2006) possibly due to glutamate excitotoxicity (Kujawa and Liberman, 2015; Puel et al., 1991). Interestingly, this seems to affect high threshold fibers (critical for hearing in noisy environments) to a greater extent (Furman et al., 2013). Experiments employing noise exposures leading to only temporary ABR or DPOAE threshold shifts, but to a permanent attenuation of CAP and ABR wave I amplitude, showed that a vast loss of functional synapses underlies these changes (Kujawa and Liberman, 2009). It is possible that in humans, excitotoxic synaptic loss would hinder hearing when background noise is present; but evidence is only emerging (Moser and Starr, 2016). Finally, degeneration of SGNs can also occur after noise insult and in ageing, occurring either after HC loss (Sha et al., 2008) or independently of this (Kujawa and Liberman, 2006). After noise trauma, SGN death seems to proceed slowly, over months or years, and is preceded by the loss of their afferent neurites. Due to the selective loss of high threshold fibers, this might not affect performance at threshold detection tests (Liberman, 2017) and thus, it is termed hidden hearing loss (Schaette and McAlpine, 2011).

\subsection{Current strategies to confront SNHL}

When hearing fails, auditory prostheses can be used to partially overcome the dysfunction. Current strategies are fundamentally based on three types of auditory prostheses.

Hearing aids amplify the sound signal and are primarily indicated in mild to moderate forms of SNHL, as well as in conductive hearing loss (Johnson et al., 2018). In more severe cases, but when spike generation and conduction in the auditory nerve remain, the cochlear implant (CI) is the option of choice. CIs typically enable open speech understanding (Wilson and Dorman, 2008; Zeng et al., 2008) and thus, they are considered the most successful neural prostheses in terms of function restoration and in number of recipients, with around 500,000 users (Wrobel et al., 2018). CIs are electrical devices that bypass the dysfunctional sensory epithelia by directly stimulating SGNs. Sounds are first captured by an external microphone and digitally processed, 
then, a transmitter sends acoustic information to internal additional electronics that control the activation of an electrode array placed in scala tympani. Several independent channels in the electrode array allow to stimulate SGNs along the tonotopic map (Lenarz, 2018). However, CIs cannot cover the whole frequency range used in normal hearing due to several reasons. The decreasing volume available in scala tympani and its coiled shape limits the depth of insertion, thus reducing the extent of stimulation (Wilson and Dorman, 2008). Moreover, due to the high conductivity of perilymph and the relative long distance between the electrodes and the spiral ganglion (Wilson and Dorman, 2008) the large current spread around each electrode contact stimulates neighboring off-target neurons ( $\mathrm{Fu}$ and Nogaki, 2005; Kral et al., 1998), impedes more than 10 non-overlapping channels (Friesen et al., 2001) and limits the output dynamic range ( $<10 \mathrm{~dB})$ (Zeng et al., 2008). This results in poor understanding of speech in background noise and hampers music appreciation (Moser, 2015a). However, despite these shortcomings, the strong electrical stimulation provided by the CI is even able to activate the auditory pathway in some cases that involve SGN disorders (Giraudet and Avan, 2012).

In cases where the auditory nerve is lost (mostly after acoustic neuroma surgery in patients with neurofibromatosis type 2), the auditory brainstem implant (ABI) restores some hearing percepts by providing electrical stimulation to the cochlear nucleus (Colletti et al., 2012). However, the fact that only a small fraction of the $<2,000$ implanted patients worldwide have reported significant speech understanding and the increased risks of craniotomy have limited its use (Schwartz and Wilkinson, 2017).

\subsection{Optogenetics as a tool to confront SNHL}

The discovery of channelrhodopsins (ChRs), first identified in the green alga Chamydomonas reinhardtii (ChR1, (Nagel et al., 2002); ChR2, (Nagel et al., 2003)), marked a milestone in the history of neuroscience. These light-gated cation channels naturally function as photoreceptors to control phototaxis in green algae (Sineshchekov et al., 2002), but were essential to fulfill a long-sought achievement: the ability to precisely control the activity of well-defined populations of excitable cells. The functional possibilities of ChR2 were demonstrated first in vitro in cultured neurons (Boyden et al., 2005) and then in vivo via lentiviral ChR2 delivery to the vibrissal motor cortex in rats to control whisker movement (Aravanis et al., 2007) and to modulate behavior in freely moving mice (Adamantidis et al., 2007). These studies paved the 
way for the expansion of optogenetics (first coined in (Deisseroth et al., 2006)). Broadly, optogenetics results from combined optical and genetic methods that allow reliable control of defined biological events, leading to gain/loss of function of those in living entities, ranging from cells to entire organisms (Deisseroth, 2010).

\section{Channelrhodopsins: structure, pore-gating kinetics, selectivity and spectral properties}

ChRs belong to the type I opsins family (also termed microbial opsins). Unlike type II opsins (animal opsins), they operate both as light sensors and ion channels. According to Yizhar et al. (2011), two more opsin classes belong to the same family: bacteriorhodopsins, light-gated outwardly-directed $\mathrm{H}^{+}$pumps (Oesterhelt and Stoeckenius, 1971) and halorhodopsins, lightdriven chloride pumps (Matsuno-Yagi and Mukohata, 1977). However, structural and functional analyses propose xenorhodopsins, light-gated inwardly-directed $\mathrm{H}^{+}$pumps, as yet another class (Shevchenko et al., 2017; Ugalde et al., 2011).

Structurally, ChRs contain seven-transmembrane domains (helices A-G) forming a pore that opens upon photoisomerization of the chromophore retinal (Yizhar et al., 2011). Retinal is covalently bound to helix $\mathrm{G}$ via a conserved lysine, forming a protonated Schiff base (Nagel et al., 2002, 2003). In the ChR1/ChR2 chimaera C1C2, the pore is formed from helices $\mathrm{A}, \mathrm{B}, \mathrm{C}$ and $\mathrm{G}$ and harbors two gates that are key for $\mathrm{H}^{+} /$cation selectivity. The internal gate is formed by glutamate residues E82 and E83 interacting with H134, while the central gate is flanked by N258, S63 and E90 (Kato et al., 2012).

The central gate opens within nanoseconds upon retinal photoisomerization, followed by a series of rearrangements that open the internal gate and render the channel conductive in the submillisecond scale $\left(\tau_{\mathrm{on}}\right)$ (Kuhne et al., 2015; Lórenz-Fonfría et al., 2015). Spectroscopy studies suggest the coexistence of light- and dark-adapted photocycles based on the syn-/antiisomerization of the chromophore, respectively. The light-adapted cycle open state (O2) is less permeable to $\mathrm{H}^{+}$and cations than its dark-adapted counterpart (O1) (Bruun et al., 2015). As O2 is gradually occupied under prolonged illumination, photocurrent adapts from the initial $\left(\mathrm{I}_{0}\right)$ to a stationary amplitude $\left(\mathrm{I}_{\mathrm{s}}\right)$. (Schneider et al., 2013). The O1/O2 ratio (as the opening and closing kinetics) is pH- and voltage-dependent and also very heterogeneous among ChRs (Mattis et al., 2011). After light-off, photocurrents outlast illumination, due to ChRs still completing their photocycle, for a time interval that varies greatly among ChRs $\left(\tau_{\text {off }}\right)$. In ChR2, $\tau_{\text {off }} \sim 10 \mathrm{~ms}$ (Gunaydin et al., 2010), what hampers temporal fidelity at high stimulation rates in fast-spiking 
neurons, as SGNs. Shorter $\tau_{\text {off }}$ values are achievable by mutations in critical residues that stabilize the positive charge of the Schiff base (Schneider et al., 2015) as E123T, reducing $\tau_{\text {off }}$ to $\sim 4 \mathrm{~ms}$ (ChETA variant) and allowing spiking up to $200 \mathrm{~Hz}$ (Gunaydin et al., 2010). However, speed in ChETA comes at the cost of reduced light sensitivity, due to the effect of the shorter channel open time on the photocurrent (Yizhar et al., 2011). Mutations H132R (Nagel et al., 2005a), T159C (Berndt et al., 2011) and the ChEF ChR1/ChR2 chimera offer lower inactivation and therefore enhanced light sensitivity than ChR2, but also slower kinetics (Lin et al., 2009).

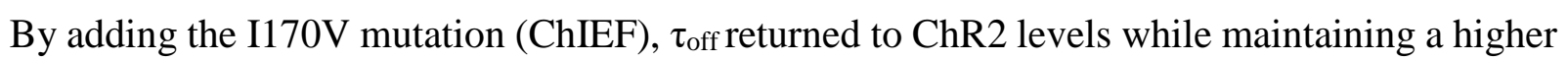
sensitivity (Lin et al., 2009). Combinations of mutations are possible and usually yield additive effects, e.g. the T159C/E123T double mutant offers larger photocurrents and faster off-kinetics (Berndt et al., 2011). In contrast, mutations at C128 and D156 slow off-kinetics up to $30 \mathrm{~min}$ and provide bistability by using blue light for activation and red-shifted light for deactivation (step-function opsins) (Berndt et al., 2008). This enhances light sensitivity by 300-fold and is useful for applications as two-photon optogenetics (Prakash et al., 2012).

The single-channel conductance of ChR2 was estimated to 40 fS (Feldbauer et al., 2009), which is orders of magnitude lower than native channels in neurons. Therefore, ChR expression levels need to be high in order to elicit physiologically-relevant currents. Chronos, isolated from Stigeoclonium helveticum, is the fastest ChR to date and was said not to trade speed off for light sensitivity, probably due to higher expression levels than other ChRs (Klapoetke et al., 2014). In terms of ion selectivity, ChRs are most permeable to $\mathrm{H}^{+}\left(\mathrm{P}_{\mathrm{H}+} / \mathrm{P}_{\mathrm{Na}+} \sim 10^{6}\right.$ (Berndt et al., 2011; Nagel et al., 2003)) while their relative conductance varies with $\mathrm{pH}$ and voltage and among cation species: $\mathrm{Na}^{+}>\mathrm{K}^{+}>>\mathrm{Ca}^{2+}>\mathrm{Mg}^{2+}$ (Nagel et al., 2003; Schneider et al., 2013). This means that under physiological conditions $\mathrm{Na}^{+}$is the main contributor to the photocurrent (in perilymph: $\left[\mathrm{Na}^{+}\right] \sim 140 \mathrm{mM}$ at $\mathrm{pH} 7.3$ (Wangemann and Schacht, 1996)). The pore of ChRs seems to lack high-affinity ion-binding sites (Nagel et al., 2003). Its selectivity mechanism rather relies on the electronegative surface of the pore, as suggested by mutations resulting in pore surface electropositivity that yielded $\mathrm{C} \mathrm{C} 2 \mathrm{Cl}^{-}$selective (Berndt et al., 2014). This mechanism also occurs in natural $\mathrm{Cl}^{-}$-selective ChRs as GtARC2 (Berndt and Deisseroth, 2015; Govorunova et al., 2015).

Moreover, the L132C mutation of ChR2 gave rise to CatCh, with enhanced light sensitivity and $\mathrm{Ca}^{2+}$ permeability. Interestingly, despite its long $\tau_{\text {off }}(\sim 16 \mathrm{~ms})$, its higher $\mathrm{Ca}^{2+}$ conductance 
speeds up hyperpolarization by opening $\mathrm{Ca}^{2+}$-dependent $\mathrm{BK}$ channels, thus allowing higher stimulation rates (Kleinlogel et al., 2011; Mattis et al., 2011).

Finally, the spectral properties of ChRs also differ. Red-shifted sensitivity seems to result from the G181S replacement in natural and artificial ChRs, leading to a biased polarity in the retinal binding pocket around the $\beta$-ionone ring of retinal (Deisseroth and Hegemann, 2017; Zhang et al., 2008). Other factors influencing red-shifted spectral properties of ChRs are retinal planarity, structural rigidity of the retinal binding pocket and protonation state of counterion residues, leading to a weaker stabilization of retinal charge (Deisseroth and Hegemann, 2017; Oda et al., 2018). Volvox ChR (VChR1) was the first natural red-shifted ChR found with a spectral peak at $\sim 550 \mathrm{~nm}$ (Zhang et al., 2008). To improve its low expression levels, membrane-trafficking/ER export signals, which were successfully used before for Natronomonas pharaonis halorhodopsin, were added (Gradinaru et al., 2010). Further chimaerization with ChR1 and ChETA mutations improved its photocurrents, resulting in C1V1, which allowed for the first red light-driven spikes (Yizhar et al., 2011). ReaChR (Lin et al., 2013), MChR1 (Govorunova et al., 2011) and bReaCHES (Rajasethupathy et al., 2015) together with Chrimson (Klapoetke et al., 2014) expanded the optogenetic toolbox. All red-shifted opsins described to date retain responsivity in blue light. Chrimson, from Chlamydomonas noctigama shows the most redshifted spectral peak at $590 \mathrm{~nm}, \sim 45 \mathrm{~nm}$ longer than other ChRs (Klapoetke et al., 2014). The same study presented the faster variant ChrimsonR (K176R), with $\tau_{\text {off }} \sim 16 \mathrm{~ms}$. The crystal structure of Chrimson revealed that its retinal binding pocket resembles light-driven ionic pumps as bacteriorhodopsin, while its ion-conduction pathway is more similar to other ChRs (Oda et al., 2018). Due to its red-shifted activation spectrum, Chrimson enables to trigger photocurrents even with high intensities of far-red light up to a maximum of $720 \mathrm{~nm}$, far beyond the limits of other opsins. Hence, a number of researchers have used Chrimson in combination with other blue light-gated ChRs to perform dual color optogenetics in model organisms as $C$. elegans (Schild and Glauser, 2015) and mouse (Kampasi et al., 2018).

\section{Cochlear optogenetics}

As described above, current CI strategies have major shortcomings that result in a poor quality of sound percepts in CI users. In contrast, cochlear optogenetics bears the potential to overcome these weaknesses. Light can be directed and focused, allowing for a better spatial confinement onto targeted SGN populations, thereby reducing the spread of excitation as compared to 
electrical stimulation (Hernandez et al., 2014a; Jeschke and Moser, 2015; Weiss et al., 2016). In the future optical CI (oCI), this would reduce channel cross-talk and promises a much higher number of independent stimulation channels to effectively increase frequency resolution (Hernandez et al., 2014a). Similarly, the oCI is also expected to expand the dynamic range of coding due to i) the smaller spread of excitation, ii) the heterogeneous opsin expression levels and iii) the differences in position relative to the emitter among SGNs, which could mimic the different thresholds observed for acoustical stimulation (Jeschke and Moser, 2015; Schoenenberger et al., 2008). However, cochlear optogenetics needs to make use of ChRs that are fast enough to maintain a high temporal precision of auditory coding (Weiss et al., 2016). To render the auditory pathway sensitive to light, ChRs need to be heterologously expressed in SGNs - cochlear optogenetics - or alternatively in other neurons from the ascending auditory pathway as in cochlear nucleus neurons (Hight et al., 2015; Shimano et al., 2013). The nonintegrating, low immunogenic adeno-associated virus (AAV) seems to be the best suited option for ChR transgene delivery and for translation of cochlear optogenetics to the clinical level, as they were in the case of the retina (Dalkara et al., 2016). Indeed, there are two ongoing clinical trials on AAV-mediated optogenetics for vision restoration in patients with retinitis pigmentosa (Simunovic et al., 2018). One of them employs AAV2-ChR2 for advanced retinitis pigmentosa and is currently in phase I/II (ClinicalTrials.gov, 2015; identifier: NCT02556736). A second one was registered in 2017 and has not yet undertaken. This study aims to use AAV2.7m8CAG-ChrimsonR-tdTomato and biomimetic Visual Interface Stimulating Glasses for nonsyndromic retinitis pigmentosa (ClinicalTrials.gov, 2017; identifier: NCT03326336).

In order to be applied in auditory system prosthetics, ChRs ideally require (1) fast kinetics to mimic the fast physiological firing of SGNs, (2) high light sensitivity to trigger large photocurrents and (3) a red-shifted action spectrum to minimize the risks of phototoxicity and reduce light scattering (Moser, 2015a).

The proof-of-concept study, carried out in our laboratory (Hernandez et al., 2014a), employed transgenic mice expressing ChR2 under the Thy1.2 promoter and trans-uterine otocyst injections of AAV2/6 for expression of CatCh under the human synapsin (hSyn) promoter. Both strategies provided enough expression levels to drive auditory activity with low irradiances in the range of few $\mathrm{mW} / \mathrm{mm}^{2}$. Optogenetic activation of the auditory pathway was demonstrated using single-channel micro light-emitting diodes ( $\mu$ LEDs) and blue laser-coupled optical fibers. 
By recording optically-driven ABRs (oABR), the population response was measured, remaining after deafening and growing with stimulus intensity, while maximal stimulation rates were modest $(<80 \mathrm{~Hz})$. Activity in the inferior colliculus reflected a lower spread of excitation than that of monopolar electrical stimulation and recordings of single auditory nerve fibers showed a lower spike jitter than that of acoustical clicks, but larger than in response to electrical stimulation. This work further showed that optogenetic stimulation partially restored auditory activity in a mouse model lacking functional otoferlin. Next steps after this study included developing a postnatal injection protocol for cochlear optogenetics (Akil et al., 2012) and using faster ChRs.

The first report on cochlear optogenetics in adult animals was published recently and used AAV2/6-CatCh-YFP (Wrobel et al., 2018). This work further provided the first behavioral evidence of hearing restoration using cochlear optogenetics. Challenges prior to clinical translation are, however, still manifold. Energy requirements need to be reduced, biosafety assessments in the long term need to be performed, as well as scaling up to larger animal models and non-human primates. On the technical side, suitable and miniaturized light sources need to be developed, and finally and most importantly, a higher performance of the oCI compared to eCIs needs to be proven (Weiss et al., 2016). Nevertheless, results are promising and the road for cochlear optogenetics has only begun. Further insight will be discussed in the next chapters.

\subsection{Aim of this work}

This work primarily aims at characterizing optogenetic stimulation of the auditory nerve using the fast-gating opsins Chronos-ES/TS and f-Chrimson expressed in SGNs. Given the low temporal bandwidth of optogenetic coding using ChR2 in the auditory system, with responses vanishing below $100 \mathrm{~Hz}$, I evaluated the ability of these two ChRs to improve spike fidelity and temporal precision of spiking in SGNs, thereby bringing them closer to physiological levels. Hence, I used electrophysiological approaches in vivo to estimate the performance of the auditory system in response to optogenetic stimulation. I used oABR recordings with varying stimulus parameters (laser power, pulse duration and stimulation rate) to characterize the population response and define the limits of the system's performance. Moreover, opticallyevoked single-unit recordings provided a higher definition and enabled me to estimate the extent of spike probability and temporal precision at increasing pulse rates. Additionally, recordings at the single neuron level allowed me to investigate SGN responses at varying light intensities and 
thus infer illumination thresholds of excitation as well as the dynamic range of optogenetic coding. 


\section{Ultrafast optogenetic stimulation of the auditory pathway by targeting-optimized Chronos}

Keppeler, D.*, Martins Merino, R.*, Lopez de la Morena, D.*, Bali, B*., Huet, A.T.\#, Gehrt, A, Wrobel, C, Subramanian, S, Dombrowski, T., Wolf, F., Rankovic, V., Neef, A. and Moser, T.

*, ${ }^{\#}$ These authors contributed equally to the work

EMBO J, Accepted manuscript

doi: 10.15252/embj.201899649

Detailed author contribution of D. Lopez de la Morena:

- Experimental work and analysis:

○ Initial cloning of pAAV-hSyn-Chronos-ES/TS-EYFP (together with S.S.) (Fig. 3.2A)

O oABR recordings from mice injected with AAV-PHP.B-Chronos-ES/TS-EYFP (together with D.K.) (Fig. 3.5)

○ In vivo recordings from single SGNs (Fig. 3.6 and Fig. EV2)

- Preparation of the manuscript (together with the other authors)

Copyright notice:

This material is reprinted by permission from EMBO Press in accordance to their copyright and licensing regulations. Reprinted by permission from Wiley-VCH Verlag GmbH \& Co. KGaA: ( $)$ owned by the authors, 2018. 


\section{Ultrafast optogenetic stimulation of the auditory pathway by targeting-optimized Chronos}

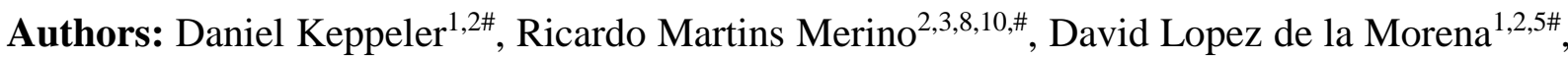
Burak Bali ${ }^{1,2,6,0}$, Antoine Tarquin Huet ${ }^{1,3,5, \circ}$, Anna Gehrt ${ }^{1,7}$, Christian Wrobel $^{1,7}$, Swati Subramanian $^{1,2}$, Tobias Dombrowski ${ }^{1, \S}$, Fred Wolf ${ }^{4,7,8,10,11}$, Vladan Rankovic ${ }^{1,6^{*}}$, Andreas Neef ${ }^{3,8,11^{*}}$, Tobias Moser $1,2,3,5,7,9,10^{*}$

\footnotetext{
Affiliations:

${ }^{1}$ Institute for Auditory Neuroscience and InnerEarLab, University Medical Center Göttingen, Germany.

${ }^{2}$ Göttingen Graduate School for Neurosciences and Molecular Biosciences, University of Göttingen, Germany.

${ }^{3}$ Biophysics of Neural Computation Group, Bernstein Center for Computational Neuroscience Göttingen, Germany.

${ }^{4}$ Bernstein Center for Computational Neuroscience Göttingen, Germany.

${ }^{5}$ Auditory Neuroscience and Optogenetics Laboratory, German Primate Center, Göttingen, Germany.

${ }^{6}$ Restorative Cochlear Genomics group, Auditory Neuroscience and Optogenetics laboratory, German Primate Center, Göttingen, Germany.

${ }^{7}$ Collaborative Research Center 889, University of Göttingen, Göttingen, Germany.

${ }^{8}$ Neurophysics Group, Max Planck Institute for Dynamics and Self-Organization, Göttingen, Germany.

${ }^{9}$ Center for Nanoscale Microscopy and Molecular Physiology of the Brain, Göttingen, Germany.

${ }^{10}$ Max Planck Institut for Experimental Medicine, Göttingen, Germany.

${ }^{11}$ Campus Institute for Dynamics of Biological Networks, Göttingen, Germany.

\#, ${ }^{\circ}$ equal contribution

${ }^{\S}$ Department of Otorhinolaryngology, Head and Neck Surgery, Ruhr University Bochum, St. Elisabeth Hospital, Bochum, Germany.

*To whom correspondence should be addressed:

Dr. Vladan Rankovic; Dr. Andreas Neef; Prof. Dr. Tobias Moser.
} 


\subsection{Abstract}

Optogenetic tools, providing non-invasive control over selected cells, have the potential to revolutionize sensory prostheses for humans. Optogenetic stimulation of spiral ganglion neurons (SGNs) in the ear provides a future alternative to electrical stimulation used in cochlear implants. However, most channelrhodopsins do not support the high temporal fidelity pertinent to auditory coding because they require milliseconds to close after light-off. Here, we biophysically characterized the fast channelrhodopsin Chronos and revealed a deactivation time constant of less than a millisecond at body temperature. In order to enhance neural expression, we improved its trafficking to the plasma membrane (Chronos-ES/TS). Following efficient transduction of SGNs using early postnatal injection of the adeno-associated virus AAV-PHP.B into the mouse cochlea, fiber-based optical stimulation elicited optical auditory brainstem responses (oABR) with minimal latencies of $1 \mathrm{~ms}$, thresholds of $5 \mu \mathrm{J}$ and $100 \mu \mathrm{s}$ per pulse, and sizable amplitudes even at $1000 \mathrm{~Hz}$ of stimulation. Recordings from single SGNs demonstrated good temporal precision of light-evoked spiking. In conclusion, efficient virus-mediated expression of targeting-optimized Chronos-ES/TS achieves ultrafast optogenetic control of neurons.

\subsection{Introduction}

Since the discovery of channelrhodopsins (ChRs, (Nagel et al., 2002, 2003) and the application of these light-gated ion channels for controlling excitable cells (Boyden et al., 2005), the concept of optogenetics has revolutionized the life sciences (Adamantidis et al., 2015; Kim et al., 2017). Application of optogenetics to restore sensory function in the immune-privileged eye and the ear are thought to have a fair chance of clinical translation (Jeschke and Moser, 2015; Sahel and Roska, 2013). Indeed, AAV-mediated optogenetics for vision restoration has recently entered a first clinical trial in a dose-finding effort (ClinicalTrials.gov, 2015; Identifier: NCT02556736) while neural coding of visual information can likely be achieved with ChRs that deactivate within several milliseconds (Busskamp et al., 2012), faster ChRs are required for sound coding in spiral ganglion neurons of the ear (SGNs) that spike at hundreds of $\mathrm{Hz}$ with sub-millisecond precision (Jeschke and Moser, 2015).

If such ultrafast optogenetic control of neural activity was available, it would serve auditory research and could fuel the development of future optical cochlear implants (oCIs). Clinically, 
this is highly relevant as approximately 466 million people-over 5\% of the world's population — suffer from a disabling hearing impairment (WHO, 2018) and we are still lacking causal therapies for the most common form; sensorineural hearing impairment. Consequences are impaired communication, often social isolation, depression, and reduction in professional capabilities. As of today, partial restoration of auditory function by hearing aids and electrical CIs (eCI) represent the options of choice for rehabilitation in sensorineural hearing impairment, which results from cochlear dysfunction or degeneration. The eCI bypasses dysfunctional or lost cochlear hair cells via direct electric stimulation of SGNs and, with most of the approximately 500.000 users achieving open speech comprehension, is considered the most successful neuroprosthesis (Lenarz, 2018; Zeng, 2017). Nonetheless, there is an urgent need for further improvement of the CI. The biggest bottleneck of the eCI is the poor spectral resolution of coding that arises from the wide spread of current around each electrode contact (Kral et al., 1998). Using light for stimulation in oCI is one of the present developments to improve spectral coding by CIs, as light can be better spatially confined than electric current (e.g. Richter et al, 2011; Hernandez et al, 2014). One of the implementations used optogenetic stimulation of SGNs for a first proof-of-principle study on activation of the auditory pathway up to the midbrain (inferior colliculus, IC), demonstrating a lower spread of cochlear excitation for fiber-based oCI than for monopolar eCI (Hernandez et al., 2014a). However, the temporal fidelity of ChR2mediated optogenetic control of SGN firing seemed limited; auditory brainstem responses broke down even below $100 \mathrm{~Hz}$ of stimulation. Higher temporal fidelity of optogenetic SGN stimulation might be achieved when using faster ChRs such as Chronos (Klapoetke et al., 2014), the newly engineered Chronos mutant ChroME (Mardinly et al., 2018) or fast Chrimson mutants (Mager et al., 2018).

Therefore, characterizing and optimizing fast $\mathrm{ChRs}$ is of great importance for fast spiking neurons in the auditory system, but also in the somatosensory system, cerebellum, and a wide range of inhibitory circuits. Here, we targeted Chronos, the fastest ChR reported so far, and first dissected its gating by patch-clamp recordings of photocurrents. Towards its application for optogenetic stimulation of mouse SGNs we optimized Chronos, the viral vector and virus injection approach for achieving high plasma membrane expression. As described for another opsin (Gradinaru et al., 2010) we appended sequences for improved exit from the endoplasmic reticulum (ES) (Ma et al., 2001; Stockklausner et al., 2001) and trafficking to the plasma membrane (TS) (Hofherr, 2005) to Chronos (Chronos-ES/TS) and performed postnatal 
injections of AAV-PHP.B serotype (Deverman et al., 2016), which drove highly efficient Chronos-ES/TS expression. We demonstrate by recordings of oABR and single SGN firing that Chronos-ES/TS enables ultrafast stimulation of the auditory pathway.

\subsection{Results}

\subsubsection{Chronos undergoes sub-millisecond on/off transitions at $36^{\circ} \mathrm{C}$}

The kinetic properties of ChRs are best studied in cells with little background conductance to characterize the light-induced conductance in isolation. To this end, we used human embryonic kidney cells 293T (HEK-293T cells) expressing Chronos or ChR2 (Methods). We clamped the membrane voltage to $-60 \mathrm{mV}$ where any light-induced change in the conductance is then linearly reflected in a change of the pipette current. First, we compared gating kinetics of ChR2 and Chronos at a low light intensity of $0.27 \mathrm{~mW} \mathrm{~mm}^{-2}$, far below the half maximal activation. At $22^{\circ} \mathrm{C}$, we found activation and de-activation time constants (mean \pm s.e.m.) of $\tau_{\mathrm{act}}=4.9 \pm 0.3 \mathrm{~ms}, \quad \tau_{\text {deact }}=9.4 \pm 1.0 \mathrm{~ms} \quad(\mathrm{n}=6) \quad$ for $\mathrm{ChR} 2$ and $\tau_{\mathrm{act}}=1.5 \pm 0.1 \mathrm{~ms}$, $\tau_{\text {deact }}=3.0 \pm 0.2 \mathrm{~ms}(\mathrm{n}=21)$ for Chronos (Figure 3.1A). When increasing the temperature to $36^{\circ} \mathrm{C}$, activation and deactivation accelerated, with $\tau_{\text {act }}=0.58 \pm 0.02 \mathrm{~ms}$ and $\tau_{\text {deact }}=0.76 \pm 0.05 \mathrm{~ms}(\mathrm{n}=6)$, Chronos reached the sub-millisecond range, while ChR2 gating kinetics, $\tau_{\text {act }}=2.3 \pm 0.1 \mathrm{~ms}, \tau_{\text {deact }}=3.0 \pm 0.3 \mathrm{~ms}$ ( $\mathrm{n}=6$ and 5 respectively), at $36^{\circ} \mathrm{C}$ were comparable to the values achieved by Chronos at $22^{\circ} \mathrm{C}$. Hence, already at such low light intensity, Chronos, but not ChR2, activation and deactivation should permit signal transmission with a bandwidth of several hundred Hertz. Probing the frequency bandwidth directly by applying light chirps, Chronos confers a much higher bandwidth compared to ChR2 (Figure 3.1B). The gain of chirp responses could be very well characterized by a single cut-off frequency of $24 \mathrm{~Hz}$ and $86 \mathrm{~Hz}$ for $\mathrm{ChR} 2$ and Chronos, respectively, at $22^{\circ} \mathrm{C}$ and $63 \mathrm{~Hz}$ and $150 \mathrm{~Hz}$ at $36^{\circ} \mathrm{C}$ (Figure 3.1C).

\subsubsection{Improving the plasma membrane expression of Chronos}

The above biophysical characterization had indicated Chronos as a strong candidate for optogenetic stimulation of SGNs with the required high temporal fidelity, provided sufficient plasma membrane expression can be achieved. Recent studies have shown that adding ER export and trafficking signals, isolated from a vertebrate inward rectifier potassium channel, to 

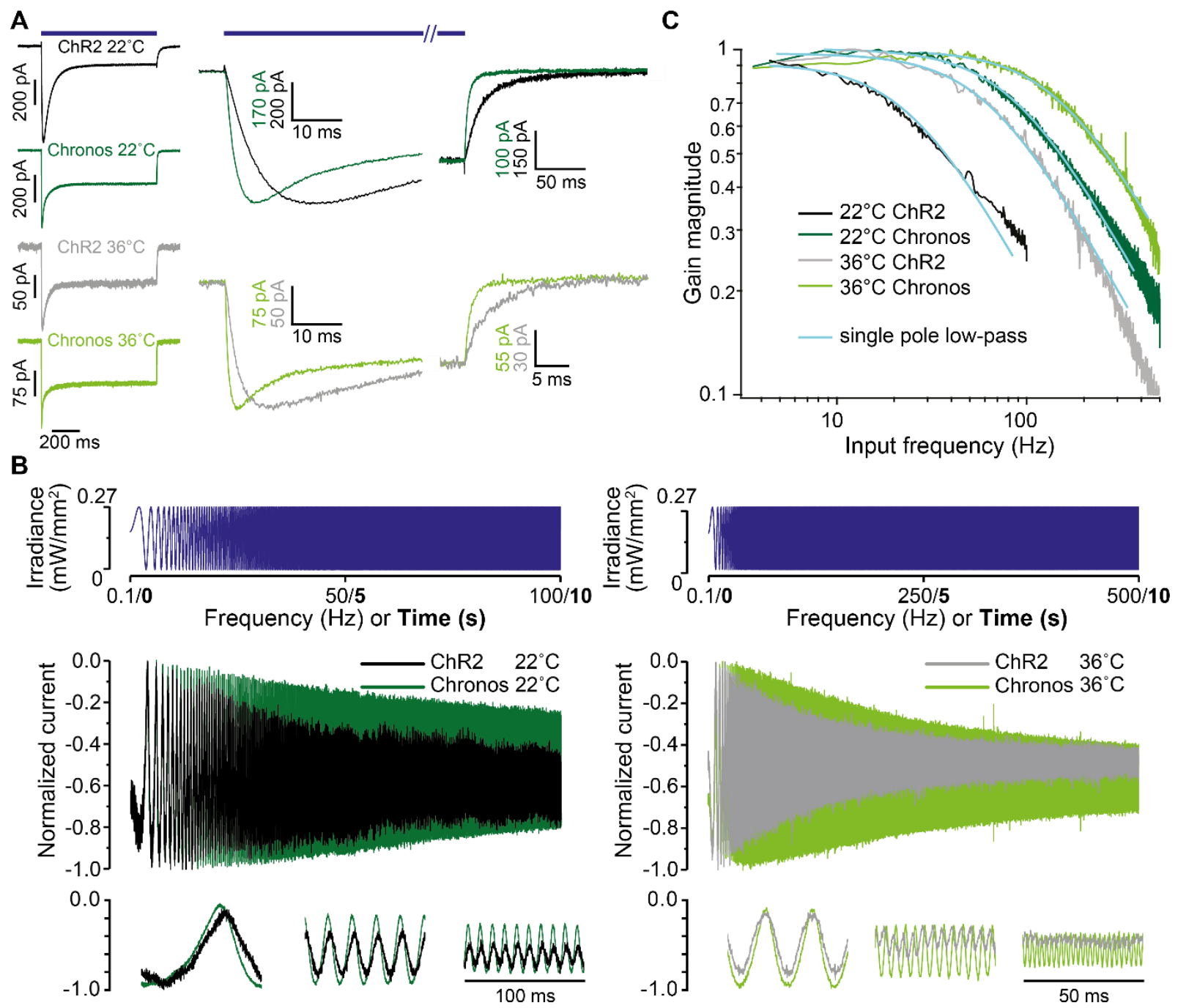

\section{Figure 3.1: Chronos mediates fast light-driven currents}

A: One second long light pulses (LED centered $480 \mathrm{~nm}, 0.27 \mathrm{~mW} \mathrm{~mm}^{-2}$ ) elicit current responses in HEK-293T cells expressing ChR2 and Chronos, tested at $22^{\circ} \mathrm{C}$ and $36^{\circ} \mathrm{C}$. Right panels show activation and deactivation at higher time resolution. B: A quasi-periodic chirp stimulus is used to directly probe the bandwidth of Chronos- and ChR2-mediated photocurrents in HEK-293T cells. Top: stimulus, middle: full response, bottom: sections from the beginning, middle and end of the response. Note the substantially larger frequency range over which Chronos currents follow the light stimulus. At $36^{\circ} \mathrm{C}$ this range is extended even further. C: Analysis of the chirp responses of HEK-293T cells as in Figure 3.1B. The current amplitude modulation is plotted against the stimulus frequency. The smooth lines represent fits to the magnitude of the transfer function of a single pole filter $a b s\left(\left(1+i f / f_{c u t}\right)^{-1}\right)$.

the cytoplasmic C-terminus of opsins promote their plasma membrane expression (Gradinaru et al., 2010). Hence, we added these sequences, here nick-named ES (Export Signal) and TS (Trafficking Signal), sandwiching EYFP, to Chronos (Chronos-ES/TS, Figure 3.2A). We first compared expression of Chronos-ES/TS and Chronos in HEK-293T cells transfected with the 
respective pAAV plasmid by immunocytochemistry. Using confocal and stimulated emission depletion (STED) microscopy we found a preferentially peripheral, likely plasmalemmal, localization of Chronos-ES/TS with some intracellular signal most likely arising from the Golgi, while the original Chronos construct was more diffusely distributed throughout the extra-nuclear intracellular space (Figure 3.2B-D). We quantified the cellular distribution of the opsin by line profile analysis (Figure 3.2C) and found a significantly greater peripheral than central immunofluorescence for Chronos-ES/TS when compared to the original Chronos construct (Figure 3.2C, D). The larger variance of the ratio of membrane and intracellular fluorescence for Chronos-ES/TS (Figure 3.2D) is likely explained by dividing by the relatively low, yet varying intracellular abundance of the opsin. In summary, the data suggests improved trafficking to the plasma membrane of Chronos-ES/TS.

Similar findings were also obtained in hippocampal neurons in culture transduced by AAV2/6 or AAV-PHP.B (Figure 3.3). This indicates that neurons, too, struggle to traffic Chronos to the plasma membrane and that this can be alleviated when adding the ES- and TS-signals, at least when tested in culture.

\subsubsection{AAV-mediated expression of Chronos and Chronos-ES/TS in mouse SGNs}

Next, we turned to expression of Chronos in mouse SGNs in vivo and, once more, compared the original Chronos and Chronos-ES/TS. We aimed to establish efficient AAV-mediated transduction of SGNs and employed the human synapsin promoter (hSyn, Figure 3.2A) that had turned out to drive efficient and specific SGN expression (Hernandez et al., 2014a). We first followed our previous protocol using transuterine injections of AAV2/6 into the otocyst of mouse embryos at embryonic day 11.5 (Figure 3.4A, upper). In most of the cases the expression of Chronos-EGFP was absent or sparse (Figure 3.4B, left and middle). Exceptionally we saw high expression levels (Figure 3.4B right). As before (Hernandez et al., 2014a), the expression, if any, was largely limited to the SGNs of the basal cochlear turn and was never seen in inner hair cells (Figure 3.4B right, inset).

Next, we moved on to early postnatal injections (Figure 3.4A, middle and lower, postnatal day (P) 5-7) into the cochlea via the round window, which had proven highly successful for transduction of hair cells (e.g. (Akil et al., 2012; Jung et al., 2015). We employed AAV-PHP.B, a novel AAV-serotype (Deverman et al., 2016) with improved efficiency of neural transduction, for expression of Chronos-ES/TS and Chronos (hSyn promoter, comparable titers, 

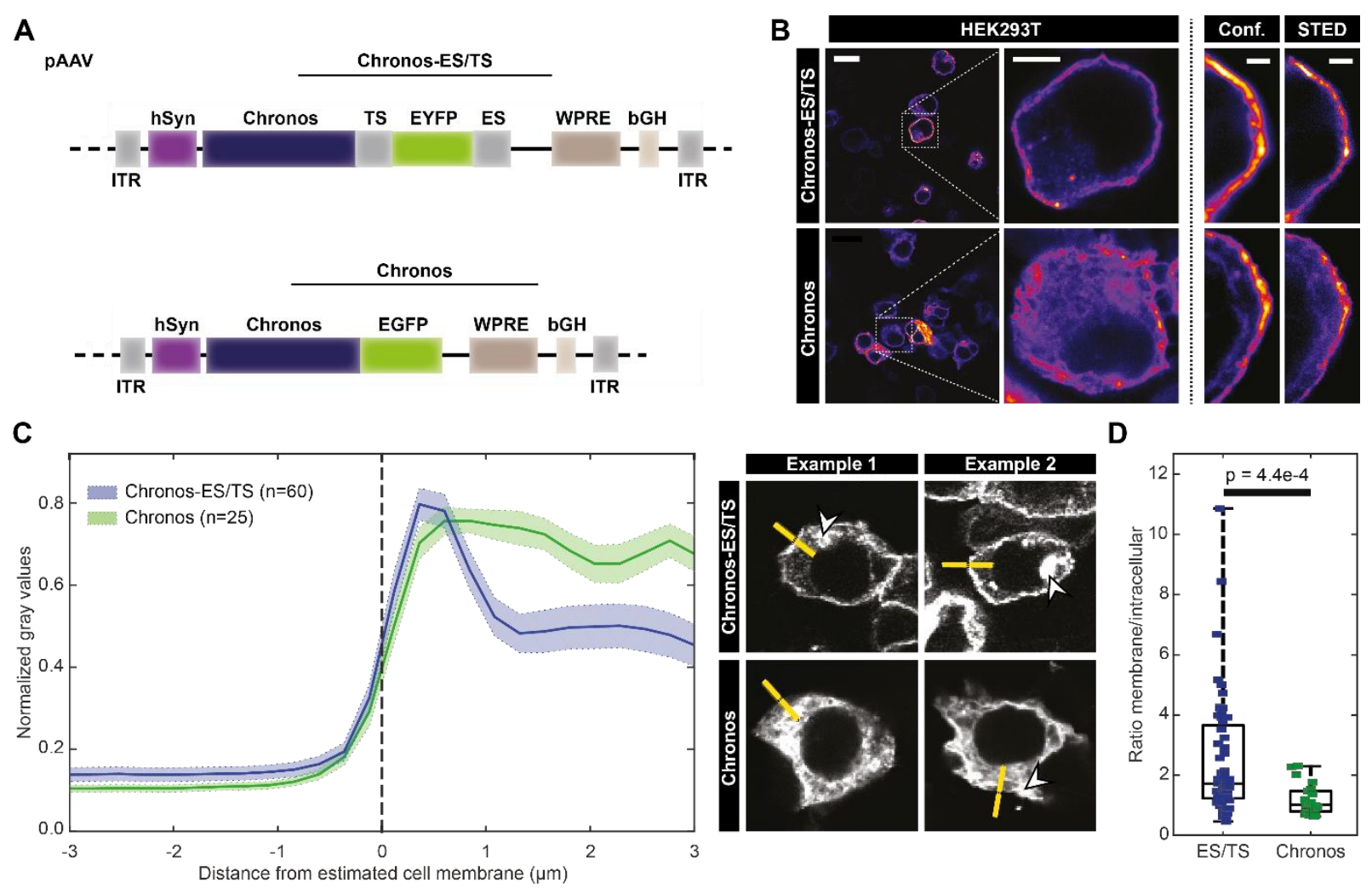

Figure 3.2: Optimizing membrane expression of Chronos by adding ER-exit and trafficking signals: HEK-293T cells

A: pAAV vector used in the study Chronos with a trafficking signal (TS), EYFP and ER export signal (ES) Chronos-ES/TS (upper) or containing the original Chronos-EGFP (Klapoetke et al., 2014), lower). In each, expression was driven by the human synapsin promoter (hSyn) and enhanced by the Woodchuck hepatitis virus posttranslational regulatory element (WPRE) and bovine Growth Hormone (bGH) polyadenylation signal (bGH poly A) sequences. ITR: inverted terminal repeats. B: Confocal and STED section of representative HEK-293T cells transfected with ChronosES/TS (upper) and Chronos (lower) and immunolabeled for FP: membranous labeling is more obvious for Chronos-ES/TS. Scale bars: 10,5 and $2 \mu \mathrm{m}$ for left, middle and right panels. C: Peak-normalized line profiles $(7.5 \mu \mathrm{m})$ centered on the estimated membrane of HEK-293T cells expressing Chronos-ES/TS (blue) or Chronos (green) as in B: mean \pm s.e.m. Chronos-ES/TS-expressing cells showed a clear peripheral, likely membrane peak, which is missing in Chronos-expressing cells. Inset shows box and whisker plot of the maximum peak at membrane. Right panels show exemplary line profile placements. One line per cell was placed perpendicular and centered to cell edge, aiming at sufficient intracellular coverage and avoiding fluorescent aggregates (arrowheads). $N$ corresponds to analyzed cells (1 sample/cell). D: Box and whisker plot of the ratio of membrane and cytoplasmic fluorescence of immunolabeled HEK-293T cells expressing Chronos-ES/TS or Chronos: Mann-Whitney U test showed significantly higher ratio in Chronos-ES/TS cells demonstrating an improved membrane expression of Chronos-ES/TS $(p=4.4 \mathrm{e}-4)$. The horizontal line within the box indicates the median, boundaries of the box indicate the 0.25 - and 0.75 -percentile, and the whiskers indicate the highest and lowest values of the results. Squares: individual data points. For details on membranous and cytoplasmic area, see Materials and Methods section. 
A

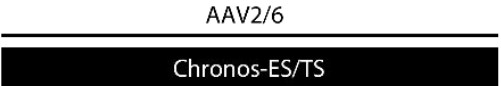

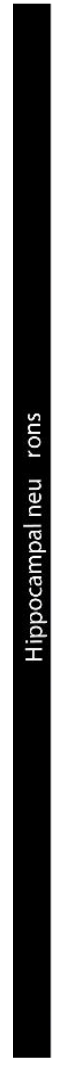
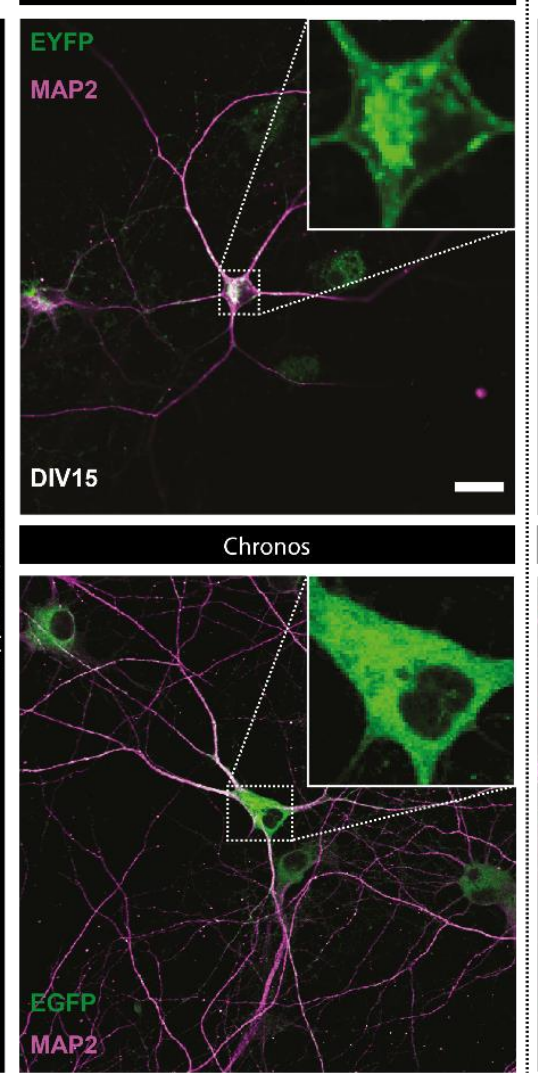

B

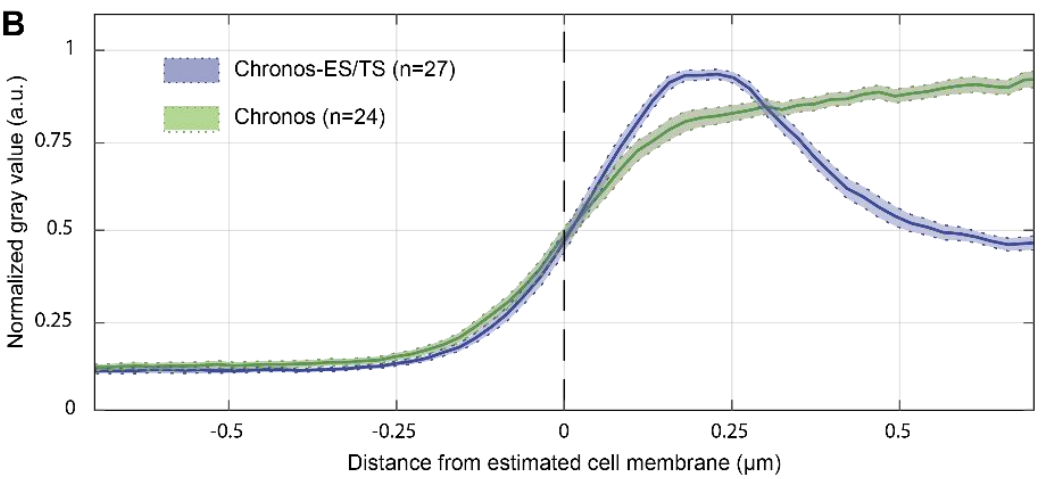

PHP.B

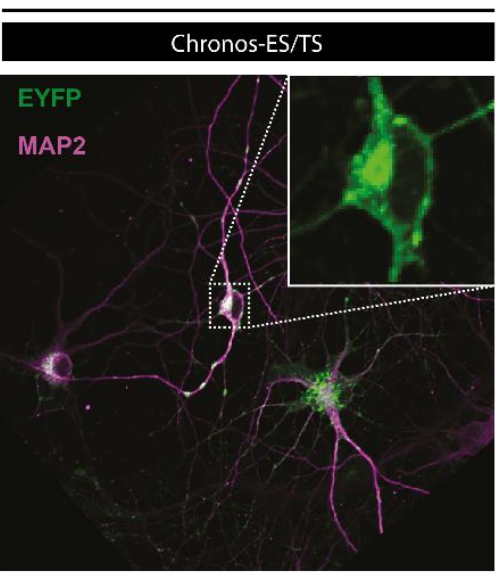

Chronos
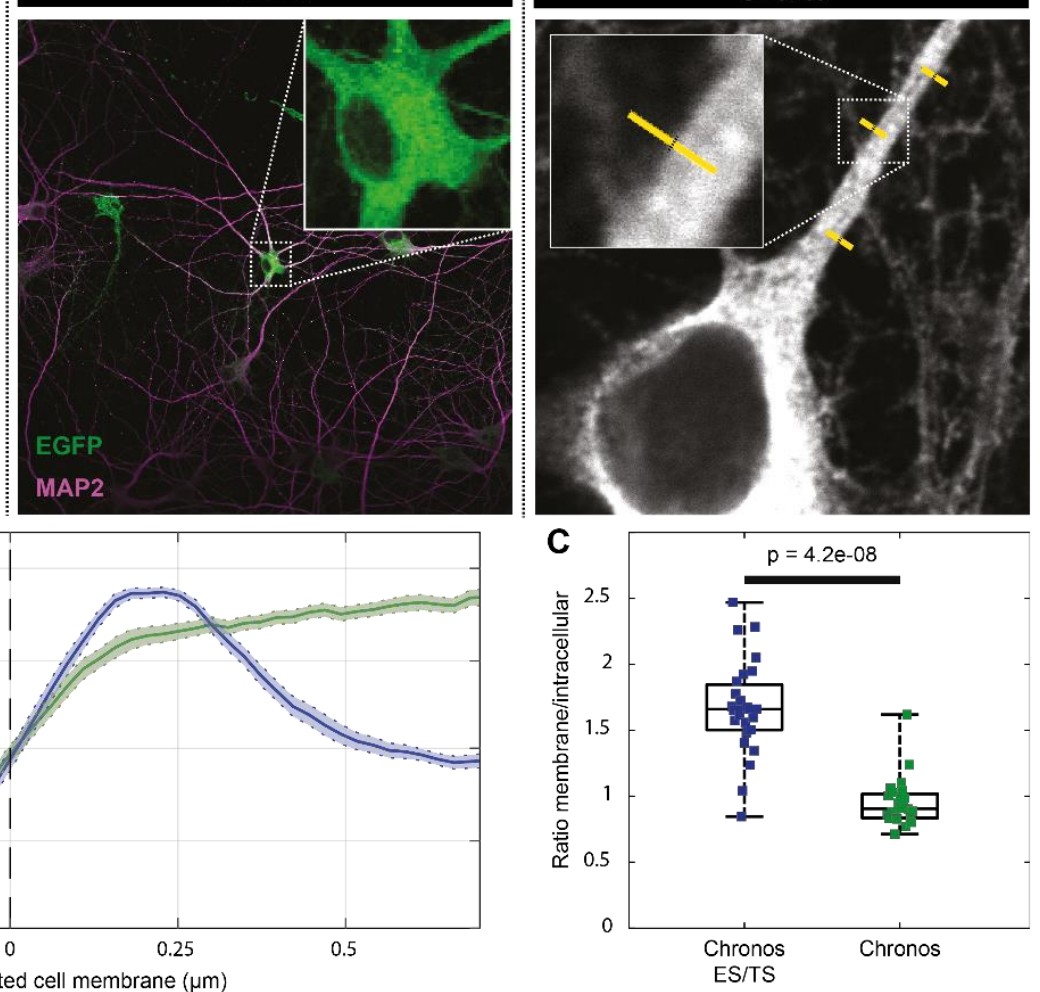

Figure 3.3: Improved membrane expression of optimized Chronos-ES/TS in hippocampal neurons

A: Hippocampal neurons infected at DIV 10 with two different versions of Chronos show very distinct expression patterns. Neurons infected with Chronos-ES/TS (upper panels) either using AAV2/6 (left) or PHP.B virus (middle) show very specific plasma membrane expression in somatic regions and proximal dendrites. Infection of neurons with Chronos (lower panels) either using AAV2/6 (left) or AAV-PHP.B (middle) showed more intracellular opsin abundance. Right panels show exemplary line profile placement (yellow). Scale bar: $50 \mu \mathrm{m}$ applies to all panels. B: Peak-normalized line profiles $(1.5 \mu \mathrm{m})$ centered on the outer cell edge of AAVPHP.B transduced hippocampal proximal dendrites expressing Chronos-ES/TS (blue) or Chronos (green) as in A: mean \pm s.e.m. Chronos-ES/TS-expressing cells showed a clear peripheral, likely plasmalemmal peak, which is missing in Chronos-expressing cells. C: Box and whisker plot of the ratio of membrane and intracellular fluorescence of immunolabeled $\rightarrow$ 
$\longleftarrow$ hippocampal neurons expressing Chronos-ES/TS or Chronos: Mann-Whitney U-test showed significantly higher ratio in Chronos-ES/TS cells demonstrating an improved membrane expression of Chronos-ES/TS $(p=4.2 \mathrm{e}-8)$. The horizontal line within the box indicates the median, boundaries of the box indicate the 0.25 - and 0.75 -percentile, and the whiskers indicate the highest and lowest values of the results. Squares: individual data points. For details on membranous and cytoplasmic area, see Materials and Methods section.

$10^{12} \mathrm{GC} \mathrm{ml}^{-1}$ ) in SGNs. Twelve out 12 AAV-PHP.B-injected mice showed substantial SGN transduction in immunohistochemistry for Chronos-ES/TS and 9 out 9 for Chronos. Postnatal injection of AAV-PHP.B drove strong expression of Chronos-ES/TS across all cochlear turns (Figure 3.4C). Similar to HEK-293T cells and hippocampal neurons in vitro, Chronos-ES/TS localized preferentially peripheral, likely plasmalemmal in SGNs in vivo, while the original Chronos construct was more diffusely distributed throughout the cell (Figure 3.4D). Once again, we quantified the cellular distribution of the opsin by line profile analysis (Figure 3.4E) and found a significantly greater peripheral than central immunofluorescence for Chronos-ES/TS when compared to the original Chronos construct (Figure 3.4E, $p=4.1 \mathrm{e}-5$ ). The difference can be appreciated by the $0.56 \mu \mathrm{m}$ more intracellular $50 \%$ FP-immunofluorescence for Chronos. We note that the cytosolic parvalbumin immunofluorescence allowed a better estimation of the cell border which was independent of the opsin expression, both advantageous when compared to HEK-293T cell analysis (Figure 3.2). Despite some differences in absolute numbers between both analyses, which are not unexpected given the different cell types, means of transfection and analysis method, both support the main observation: improved relative plasma membrane abundance of Chronos-ES/TS.

When analyzing the transduction rates in the injected (left) and non-injected (right) cochleae, we confirmed robust SGN transduction across all turns of the injected cochlea for both ChronosES/TS and Chronos (Figure 3.4F). We note that the counterstain for parvalbumin- $\alpha$, used for AAV-PHP.B injected ears, is a more general marker of SGNs than calretinin, used for AAV2/6 injected ears, which is present only in a subset of SGNs. Therefore, if anything, we would have been prone to overestimate the transduction rate for AAV2/6 injected ears, which, however, was very low. Interestingly, for both cases of AAV-PHP.B injection we also found substantial expression in the contralateral, non-injected cochleae, indicating spread of virus in the specific conditions of pressure injection into the scala tympani of the early postnatal cochlea. This spread likely occurred via the cochlear aqueduct and/or the endolymphatic ducts and the cerebrospinal 
fluid space (Lalwani et al., 1996). The density of SGNs in the injected as well as in non-injected ears were comparable (Figure 3.4G). The injected mice behaved normal as concluded from routine animal observation.

A
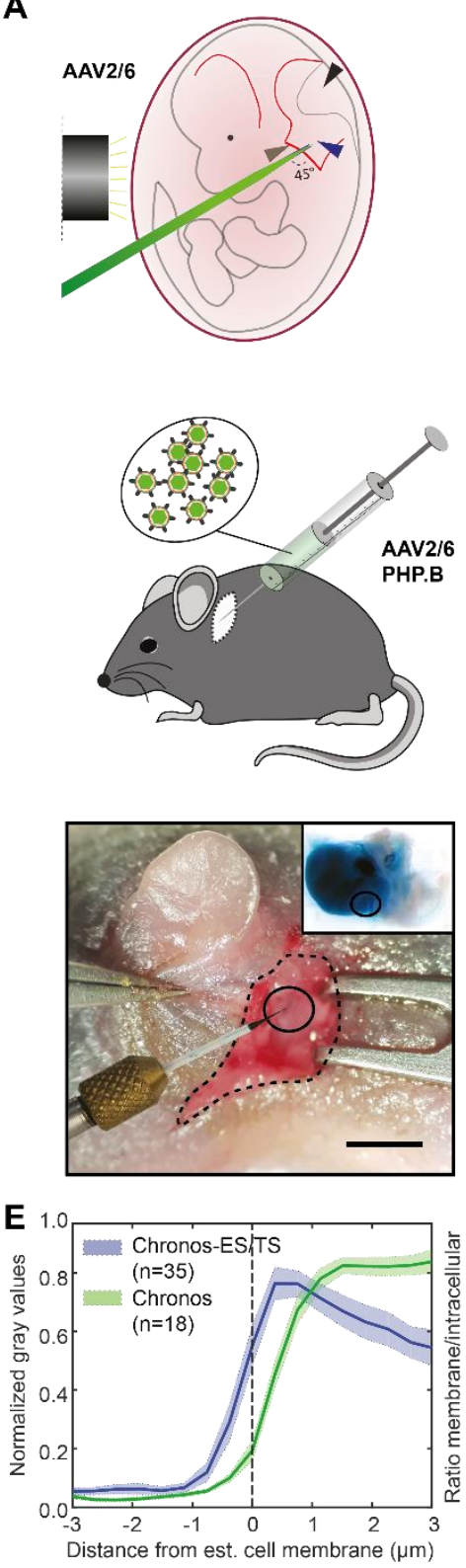

B

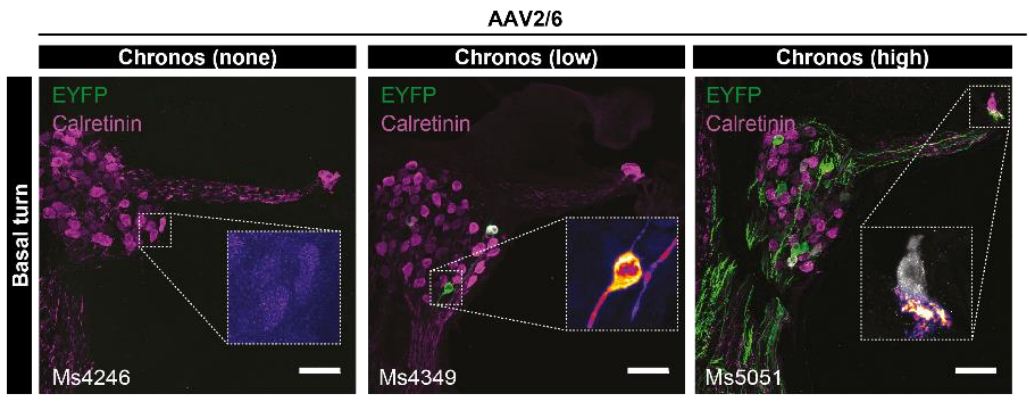

C

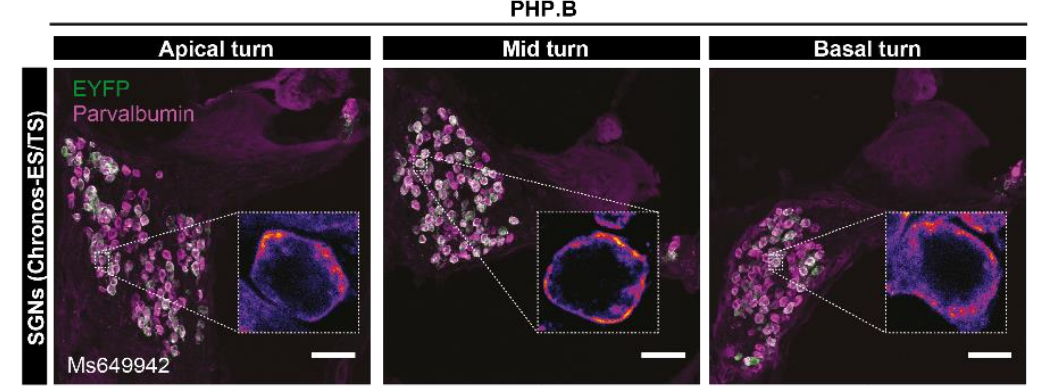

D

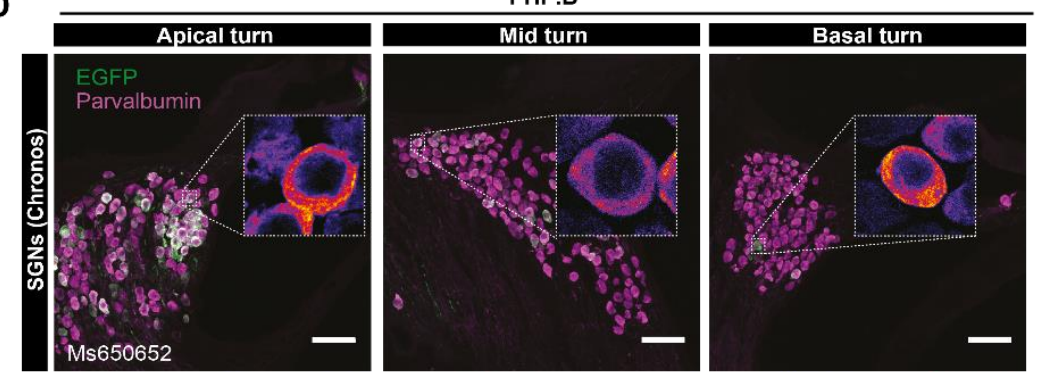

Figure 3.4: Establishing efficient expression of Chronos in SGNs: use of Chronos-ES/TS, potent AAV-PHP.B, and postnatal mode of AAV-injection

A: Upper panel: schematic representation of the viral injection into the embryonic otocyst (left: black cylinder marks the light guide used to trans-illuminate the embryo in the uterus after mobilization from the abdominal cavity, green: micropipette filled with fast green-colored AAV suspension). Middle panel: schematic representation of AAV-injection into the postnatal cochlea via the round window $(\mathrm{RW})$. Lower panel: surgical situs of a P7 mouse with $\rightarrow$ 
$\leftarrow$ retroauricular incision, graphical aid encircles the injection site). Inset shows ex-vivo cochlea just after AAV injection via RW. Scale bar: $2 \mathrm{~mm}$. B: Maximum projection of confocal images of immunolabeled mid-modiolar cochlear cryosections (exemplary sections of basal turn) of embryonically AAV2/6-Chronos-injected mice collected at 4-weeks of age. EYFP (green) marks transduced SGNs, calretinin (magenta) was used as generic marker of SGNs, scale bar: $50 \mu \mathrm{m}$. In the inset, color code for EYFP channel was changed to fire (EYFP). Left panel: most common, non-expressing example, inset shows zoom of negative SGNs. Middle panel: occasional, sparsely expressing example, inset: one out of two positive SGNs. Right panel: rare, highly expressing example, inset: negative inner hair cell (calretinin in gray), exclusive localization of EYFP in the SGN boutons and fibers. C: Postnatally AAV-PHP.B-ChronosES/TS-injected mouse (see B except where stated differently). EYFP (green) marks transduced SGNs, parvalbumin (magenta) was used as generic marker of SGNs, scale bar: $50 \mu \mathrm{m}$. High transduction rate, good membrane expression. In inset, color code for the green channel was changed to fire for better visualization. Similar to B. D: Postnatally AAV-PHP.B-Chronosinjected mouse (see C except where stated differently). Substantial SGN transduction, poor membrane expression. E: Line profile analysis of FP-immunofluorescence across the membrane of SGN somata. Traces were centered at the transition from high to low parvalbumin immunofluorescence as a proxy of plasma membrane location. Left panel: clear membrane abundance in Chronos-ES/TS (blue, mean \pm s.e.m.), but mostly intracellular localization in Chronos (green, mean \pm s.e.m.). Inset shows box and whisker plot of the maximum fluorescence at the membrane. Right panel: Box and whisker plot of the ratio of membrane and intracellular FP fluorescence for Chronos-ES/TS (left) and Chronos (right): stronger relative membrane expression in Chronos-ES/TS (Mann-Whitney U test, $p=4.1 \mathrm{e}-5$ ). Squares: individual data points. For details on membranous and cytoplasmic area, see Materials and Methods section. F: Box and whisker plot of the fraction of FP-positive SGNs (transduced out of all parvalbuminpositive SGNs) for the apical, middle and basal cochlear turn of the injected ear (colored) and contralateral, non-injected (grey) ear of Chronos-ES/TS-AAV-PHP.B and Chronos-AAVPHP.B mice. Points: individual animals plotted on top; $n$ refers to number of cochleae studied. The horizontal line within the box indicates the median, boundaries of the box indicate the 0.25 and 0.75-percentile, and the whiskers indicate the highest and lowest values of the results. G: Box and whisker of the SGN density for the apical, middle and basal cochlear turn of the injected (colored) ear and the contralateral, non-injected (grey) ear (as in F). No significant differences between Chronos and Chronos-ES/TS nor between injected and non-injected ear. Points: individual cells plotted on top, $n$ as stated in F.

\subsubsection{Chronos-mediated optogenetic neural stimulation of the auditory pathway:}

\section{transuterine injections of AAV2/6}

We performed acute recordings of oABRs using fiber-coupled laser stimulation 4-12 weeks after AAV-injection. Following posterior tympanotomy, we inserted a $50 \mu \mathrm{m}$ optical fiber through the round window (RW) to project the light of a blue laser $(\lambda=473 \mathrm{~nm})$ onto the SGNs. When oABRs were present (Figure EV1), we typically found three to five oABR peaks, which

likely reflected the synchronous activation of Chronos-expressing SGNs (first peak) and 
downstream auditory pathway (subsequent peaks). Transuterine injections of AAV2/6-Chronos did not generally support oABRs; only 3 out of dozens of injected mice (including 2 different AAV2/6 produces) showed oABRs. In these positive mice, oABR grew in amplitude and showed shorter latencies when increasing radiant flux (see inset of Figure EV1).

\subsubsection{Chronos-ES/TS enables ultrafast optogenetic stimulation of the auditory pathway: oABRs}

Postnatal injection of AAV-PHP.B reliably achieved high transduction rates (Figure 3.4), which enabled oABRs in 19 out of 20 AAV-PHP.B-Chronos-ES/TS-injected mice and 8 out of 17 AAV-PHP.B-Chronos-injected mice. Next, we performed a detailed characterization of Chronos-ES/TS-mediated optogenetic activation using oABRs and compared the results to those obtained with Chronos. oABR amplitude grew with increasing stimulus intensity (Figure 3.5A,B, $1 \mathrm{~ms}$ light pulses delivered at $10 \mathrm{~Hz}$ - averaged across 1000 repetitions), while oABR latency (i.e. the time interval between the stimulus onset and the oABR $\mathrm{P}_{1}$ wave) got shorter for Chronos-ES/TS (sign test between the threshold and highest radiant flux tested, $p=0.009$ ), which did not reach statistical significance for Chronos (Figure 3.5A,C; $p=0.11$ ). oABR thresholds and amplitudes differed between animals (shown for the first peak, $\mathrm{P}_{1}-\mathrm{N}_{1}$, Figure 3.5B). Stimuli as weak as $1.08 \mathrm{~mW}$ (duration: $1 \mathrm{~ms}$, rate: $10 \mathrm{~Hz}$ - averaged across 1000 repetitions) were sufficient to drive oABRs in one Chronos ES/TS injected mouse and $4.56 \mathrm{~mW}$ in a Chronos injected mouse. For $1 \mathrm{~ms}$ light pulses delivered at $10 \mathrm{~Hz}$, the average oABR threshold amounted to $6.58 \pm 1.08 \mathrm{~mW}$ and $13.95 \pm 3.52 \mathrm{~mW}$ for Chronos-ES/TS and Chronos, respectively ( $p=0.0367$, Mann-Whitney $\mathrm{U}$ test; $n=13$ and $n=8$ ). In most animals oABR amplitudes grew with radiant flux increasing over more than one order of magnitude (Figure 3.5B). The minimal latency of the first oABR peak $\left(\mathrm{P}_{1}\right.$, Figure 3.5C) amounted to $0.95 \pm 0.07 \mathrm{~ms}(\mathrm{n}=13)$ for Chronos-ES/TS and $1.26 \pm 0.07 \mathrm{~ms}$ for Chronos $(\mathrm{n}=8, p=0.014$, Mann-Whitney U test).

oABRs could be elicited by light pulses as short as $20 \mu \mathrm{s}$ with Chronos-ES/TS and $400 \mu \mathrm{s}$ with Chronos (irradiance: $38 \mathrm{~mW}$, rate: $10 \mathrm{~Hz}$ - averaged across 1000 repetitions, Figure 3.5D,E). oABR amplitudes grew with pulse duration up to approximately $0.6 \mathrm{~ms}$ and tended to become smaller for longer pulses, possibly due to accumulating channel inactivation and/or increasing depolarization-block of SGNs upon prolonged photo-depolarization (Figure 3.5D,E). 


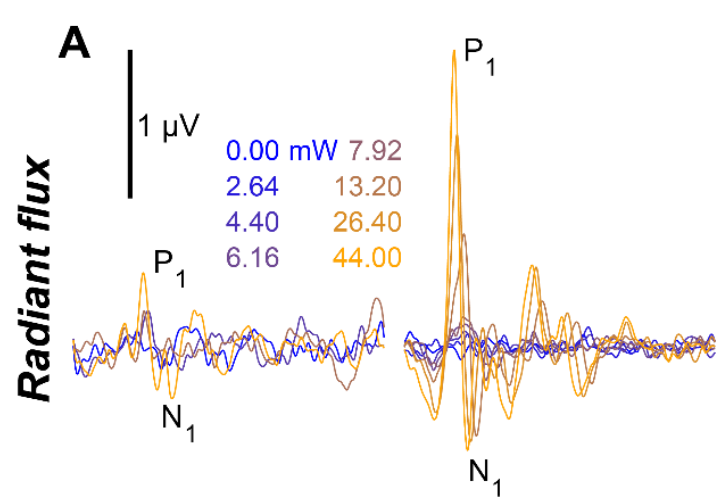

Chronos Chronos-ES/TS
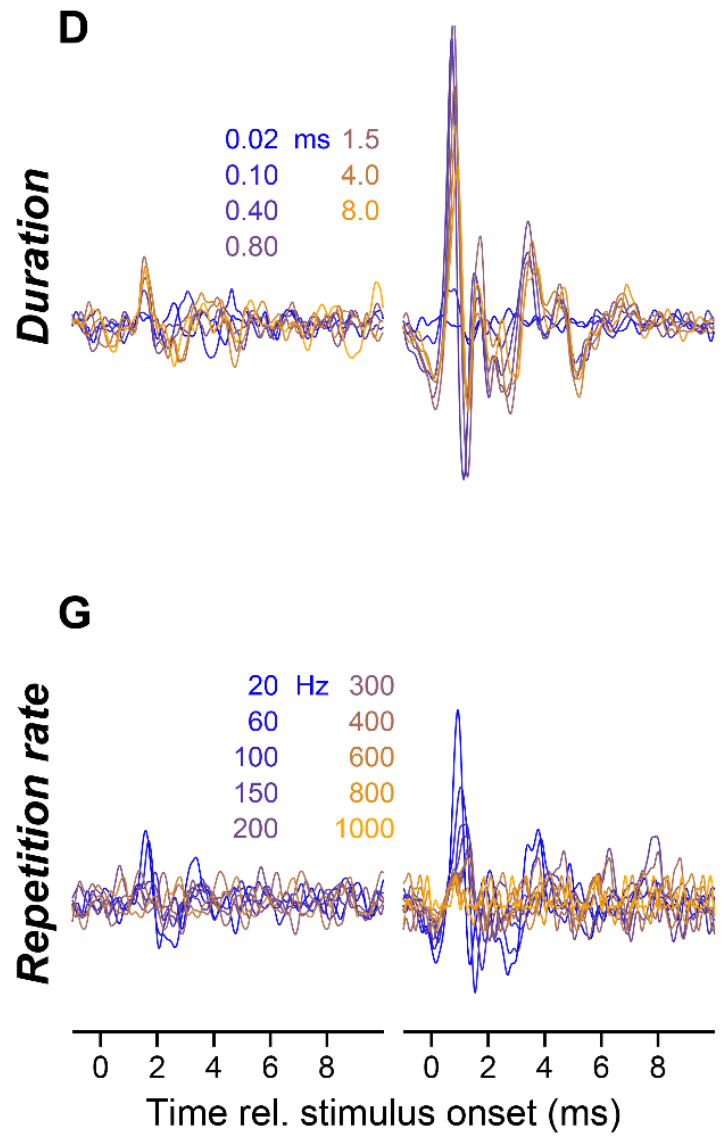
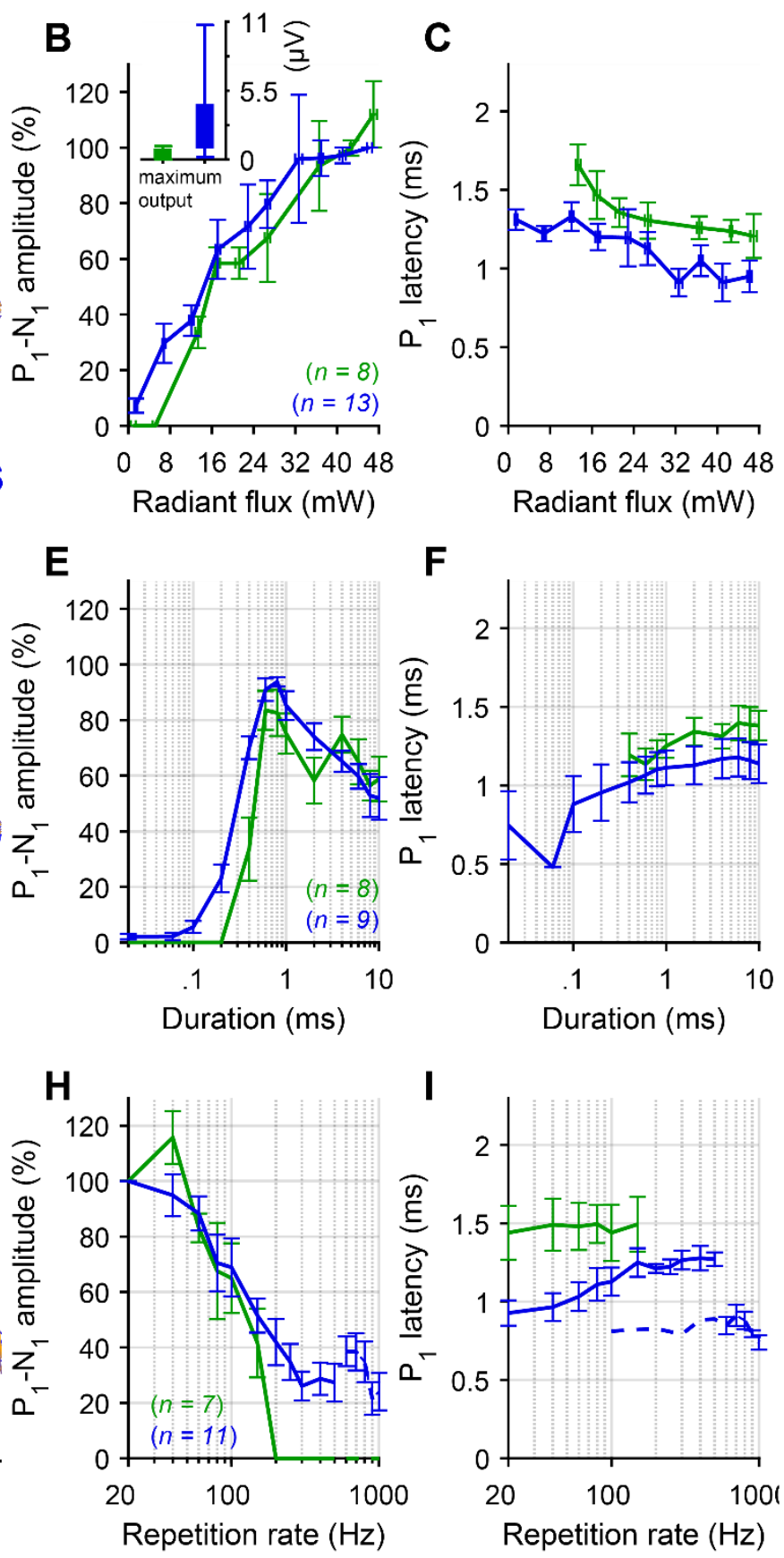

Figure 3.5: Characterizing optogenetic stimulation by recordings of oABRs

A: oABRs driven with varying radiant flux ( $1 \mathrm{~ms}$ at $10 \mathrm{~Hz}$, colors code the radiant flux in $\mathrm{mW}$ ) for exemplary mice injected with AAV-PHP.B-Chronos (left) or AAV-PHP.B-Chronos-ES/TS (right). B: Normalized $\mathrm{P}_{1}-\mathrm{N}_{1}$ amplitude of oABR as a function of light intensity for Chronos as in A (green, throughout figure) and Chronos-ES/TS (blue). Radiant flux was binned per 5 $\mathrm{mW}$ steps (horizontal error: \pm s.e.m.). Inset: maximal absolute $\mathrm{P}_{1}-\mathrm{N} 1$ amplitude of oABR (mean \pm s.e.m.) was greater for Chronos-ES/TS than for Chronos $(p-\leq 0.001$; Mann-Whitney$\mathrm{U}$ test). C: Latency of oABR $\mathrm{P}_{1}$ as a function of radiant flux as in $\mathrm{B}$. Radiant flux was binned per $5 \mathrm{~mW}$ steps (horizontal error: \pm s.e.m.). D: oABRs driven with varying stimulus duration $(10 \mathrm{~Hz}, 38 \mathrm{~mW}$, colors code the duration) for exemplary mice injected with AAV-PHP.BChronos (left) or AAV-PHP.B-Chronos-ES/TS (right). E: $\mathrm{P}_{1}-\mathrm{N}_{1}$ amplitude as a function of $\rightarrow$ 
$\longleftarrow \quad$ stimulus duration as in $\mathrm{D}$ (normalized against $\mathrm{P}_{1}-\mathrm{N} 1$ oABR amplitude. $\mathrm{F}$ : Latency of oABR $\mathrm{P}_{1}$ as a function of stimulus duration as in E. G: oABRs driven with varying stimulus rate (1 ms and $0.5 \mathrm{~ms}$ for $10-500 \mathrm{~Hz}$ and $500-1000 \mathrm{~Hz}$, respectively, $38 \mathrm{~mW}$, colors code the stimulus rate) for exemplary mice injected with AAV-PHP.B-Chronos (left) or AAV-PHP.B-Chronos-ES/TS (right). H: $\mathrm{P}_{1}-\mathrm{N}_{1}$ amplitude as a function of stimulus rate as in $\mathrm{G}$ (normalized against $\mathrm{P}_{1}-\mathrm{N}_{1}$ oABR amplitude at $20 \mathrm{~Hz}$ ). I: Latency of oABR $\mathrm{P}_{1}$ as a function of stimulus rate as in $\mathrm{H}$ : Chronos-ES/TS enables responses up to at least $1000 \mathrm{~Hz}$. Dashed line indicates latency data points obtained with a pulse duration of $0.5 \mathrm{~ms}$.

Data information: The $\mathrm{P}_{1}-\mathrm{N}_{1}$ amplitude and $\mathrm{P}_{1}$ latency were measured on the same animals. Animal count $(n)$ is stated on bottom right of panel B, E, and H. Data are expressed as mean \pm s.e.m.

Next, we tested the dependence of oABRs on the stimulus rate [duration: $1 \mathrm{~ms}$ up $500 \mathrm{~Hz}$ and $0.5 \mathrm{~ms}$ from $500 \mathrm{~Hz}$, intensity: maximum $(38-43 \mathrm{~mW})]$. When increasing stimulus rate, oABR amplitudes declined (Figure 3.5G) and latencies prolonged (Figure 3.5G,I). However, in contrast to our previous reports on: i) ChR2: where potentials were found only up to $70 \mathrm{~Hz}$ (Hernandez et al., 2014a); ii) CatCh: up to $200 \mathrm{~Hz}$, and f-Chrimson: up to $250 \mathrm{~Hz}$ (respectively (Wrobel et al., 2018; Mager et al., 2018), we could detect sizable $\mathrm{P}_{1}-\mathrm{N}_{1}$ up to stimulus rates of $500 \mathrm{~Hz}$ for Chronos (Figure 3.5G,H) and $1000 \mathrm{~Hz}$ for Chronos-ES/TS (the highest tested in our experiments, Figure $3.5 \mathrm{G}-\mathrm{H})$. $\mathrm{P}_{1}$-latency increased with higher stimulus rates in both cases.

\subsubsection{Chronos-ES/TS enables ultrafast optogenetic stimulation of the auditory pathway: recordings from single putative SGNs}

To further validate the Chronos-ES/TS-mediated SGN stimulation and evaluate the temporal fidelity of stimulation, we performed juxtacellular recordings from auditory nerve fibers (central axon of SGN) as described in (Hernandez et al., 2014a; Mager et al., 2018). In brief, we targeted glass micropipettes to where the auditory nerve enters the anteroventral cochlear nucleus $(\mathrm{AVCN})$ and searched for responses while stimulating the SGNs through the round window via an optical fiber coupled to a blue laser. We favored recordings of SGNs by deep positioning ( $>1000 \mu \mathrm{m}$ relative to the surface of the cochlear nucleus) of the pipette tip, but, given that responses to acoustic stimuli were lost upon the ear surgery, we could not safely discriminate SGNs from AVCN neurons, hence, we termed the light-responsive neurons "putative SGN". We found that the putative SGNs fired upon optogenetic stimulation with high temporal precision for stimulus rates of up to hundreds of $\mathrm{Hz}$ (Figure 3.6A, B); some neurons followed 
stimulation to some extent even up to $1000 \mathrm{~Hz}$ (Figure 3.6B). Temporal precision of firing, evaluated based on vector strength (Goldberg and Brown, 1969), see Materials and Methods, Figure 3.6C) and spike jitter (i.e., standard deviation of spike latency across trials, Figure 3.6D), was generally high, but varied across the recorded neurons.

Temporal precision and spike probability (Figure 3.6E) diminished with increasing stimulation rates, indicating that single SGNs code optogenetic information in a less reliable manner at very high stimulation rates. Spike jitter, calculated for spikes occurring in a time window comprised between two pulse onsets, increased with rate but it was typically below a millisecond for stimulus rates lower than $300 \mathrm{~Hz}$ (Figure 3.6D). At higher stimulus rates, spike jitter increased beyond the values obtained for simulated Poisson spike trains (see Materials and Methods, redshaded area, Figure 3.6D), indicating that spike synchronization with the light pulses became less reliable. The lower spike precision and limited spike probability at stimulus rates beyond $100 \mathrm{~Hz}$ observed are likely compensated by the population response, as several SGNs jointly encode information from each place of the tonotopic map (Liberman, 1978).

Light-evoked firing rate patterns differed among the putative SGNs as stimulation rates increased (Figure 3.6F). There was, in general, a linear rise of discharge rates up to $100 \mathrm{~Hz}$, followed by a constant decrease as a response to mid and high stimulation rates. Nevertheless, some putative SGNs were able to maintain moderate (and to some extent synchronized) spike rates even at pulse rates of $1000 \mathrm{~Hz}$ (fibers showing computable spike probability — see Materials and Methods - at $500 \mathrm{~Hz}: 7 / 21,33.3 \%$; at $600 \mathrm{~Hz}: 3 / 18,16.7 \%$; at $700 \mathrm{~Hz}: 3 / 18$, 16.7\%; at $800 \mathrm{~Hz}: 1 / 19,5.26 \%$; at $900 \mathrm{~Hz}: 2 / 21,9.52 \%$; and at $1000 \mathrm{~Hz} 2 / 19,10.53 \%$ ). Interestingly, the response patterns of the putative SGNs that we recorded varied across units at increasing stimulation rates (Figure EV2). We found two main types of responses; putative SGNs that rapidly adapted their spiking as stimulation rates rose (Figure EV2A), and neurons that continued spiking even at very high pulse rates (albeit with a lower frequency as compared to lower stimulation rates, Figure EV2B). 
A

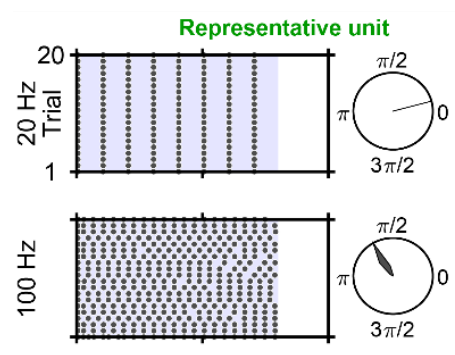

B
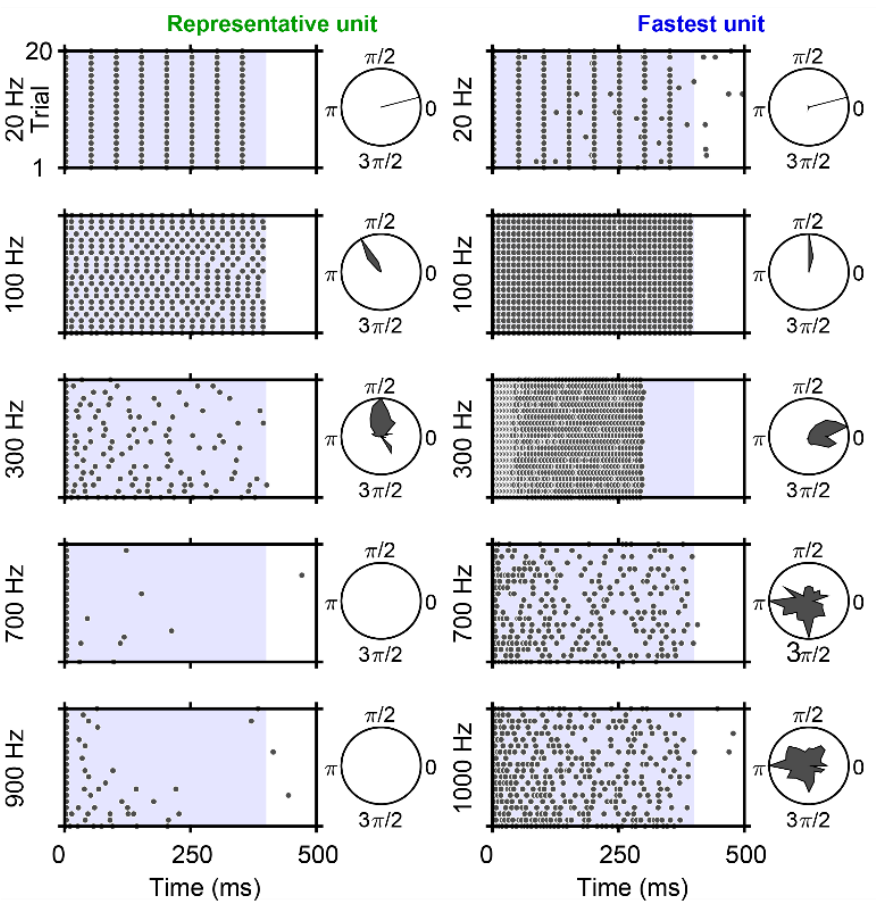

C
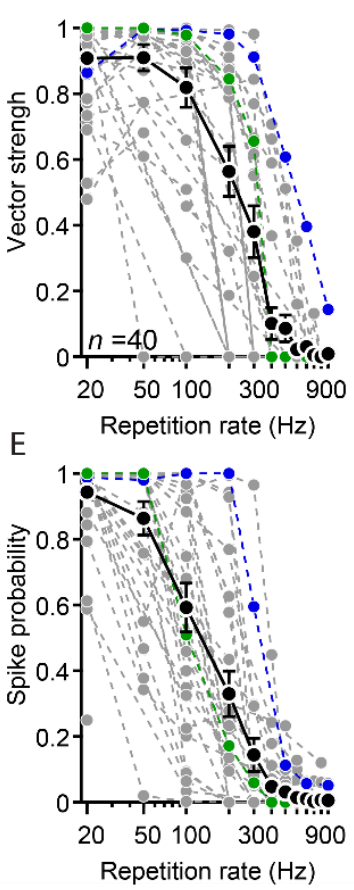

D

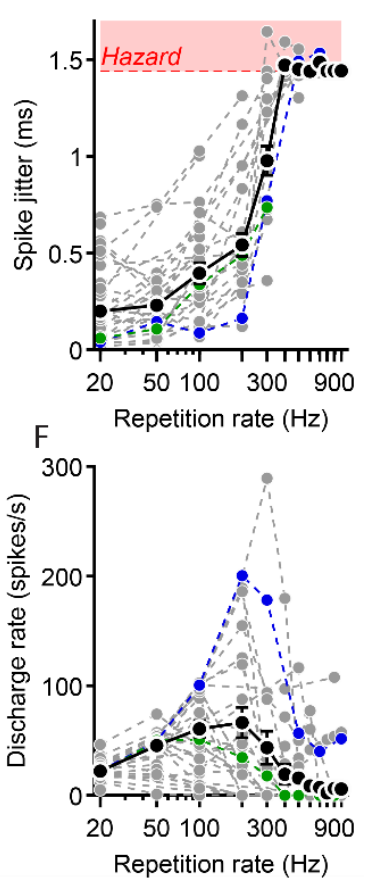

Figure 3.6: Characterizing optogenetic stimulation by juxtacellular recordings from single putative SGNs

A: Raster plots showing spiking activity from a representative putative SGN ("unit") in response to $400 \mathrm{~ms}$-long trains of laser pulses (at $30 \mathrm{~mW}, 1 \mathrm{~ms}$ for $20-600 \mathrm{~Hz}, 500 \mu \mathrm{s}$ for $\geq 700 \mathrm{~Hz}$ ) recorded at five different stimulation rates over 20 repetitions. Polar plots to the right side of raster plots show synchronicity of firing relative to the cycle between two pulse onsets. B: Same protocol and analysis of the "fastest unit" recorded, showing lower adaptation and firing even at very high stimulation rates. C-F: Quantification of the vector strength $(\mathbf{C})$, spike jitter (D), spike probability $(\mathbf{E})$ and discharge rate $(\mathbf{F})$ as a function of repetition rate of 40 putative SGNs (obtained from 6 mice). In panel D, the red-shaded area represents the hazard function obtained in response to simulated Poisson spike trains.

Data information: Black data points and lines show mean \pm s.e.m.; individual units are shown in gray. The units shown on A-B are shown in green and blue, respectively. Number of units tested for each repetition rate: $20 \mathrm{~Hz}: 40,50 \mathrm{~Hz}: 25,100 \mathrm{~Hz}: 24,200 \mathrm{~Hz}: 26,300 \mathrm{~Hz}: 21,400$ Hz: 20, 500 Hz: 21, 600 Hz: 18, 700 Hz: 18, 800 Hz: 19, 900 Hz: 21, 1000 Hz: 19.

\subsection{Discussion}

Here, we characterized and optimized Chronos for its utility in fast optogenetic control of excitable cells. We demonstrate sub-millisecond off-kinetics at physiological temperature of Chronos. Using the auditory system as a fast spiking neural model circuitry, we show that Chronos supports ultrafast control of neuronal spiking. In doing so, we solved a major shortcoming that plagued optogenetics as a stimulus modality for the cochlea; the low temporal 
fidelity of light-driven SGN-firing, caused by the slow deactivation kinetics of the ChR2 employed so far. We found that adding sequences promoting ER-exit and plasma membrane trafficking to Chronos (Chronos-ES/TS) and using postnatal injection of the powerful AAVPHP.B vector critically improved the in vivo utility. In conclusion, Chronos-ES/TS in combination with potent viral vectors such as AAV-PHP.B is a promising tool for auditory neuroscience and a candidate $\mathrm{ChR}$ for use in future optical CIs.

\subsubsection{Biophysical characterization of Chronos and improving plasma membrane expression}

Here, we compared activation and deactivation for ChR2 and Chronos and also studied the temperature dependence of gating for Chronos. In our hands Chronos deactivates about 3 times faster than ChR2 and has sub-milliseconds off-kinetics at physiological temperature with a $\mathrm{Q}_{10}$ of 2.7. This reflects short-lived open states for Chronos resulting in lower open probability, which together with the relatively poor membrane expression likely explains the challenges we faced with using the original Chronos construct for driving SGN spiking, when using the transuterine injection in the AAV2/6-hSyn vector that we previously used successfully for the ChR2 mutant CatCh (Hernandez et al., 2014a). No problems were reported in two other studies of Chronos in the auditory system using different viruses and stages of the pathway; cochlear nucleus (Hight et al., 2015) and inferior colliculus (Guo et al., 2015). A recent study using the in silico predicted ancient AAV Anc80 also achieve functional expression in the cochlea, but did not differentiate between expression in SGNs and hair cells (Duarte et al., 2018). Inspecting the Chronos-GFP expression in the cochlear nucleus (Figure 2 of (Hight et al., 2015)) and SGNs (Duarte et al., 2018) suggest a similar diffuse intracellular distribution as we observed in HEK293T cells (Figure 3.2) and hippocampal neurons (Figure 3.3) in culture as well as in SGNs in our study (Figure 3.4). Using these 3 cell types and vastly different conditions, we show a robust improvement of the plasma membrane expression of Chronos upon adding ER-export (Ma et al., 2001; Stockklausner et al., 2001) and trafficking signals (Hofherr et al., 2005) of inward rectifying $\mathrm{K}^{+}$channels. Introduction of the powerful AAV-variant AAV-PHP.B (Deverman et al., 2016) was an additional factor changing the game: even the unmodified Chronos (Klapoetke et al., 2014) enabled oABRs on a regular basis. However, when comparing the functionality of Chronos-ES/TS with enhanced membrane trafficking to Chronos, transduced by the same AAVPHP.B vector, titer, injection method and incubation time, we found increased oABR amplitudes 
and improved temporal fidelity of the Chronos-ES/TS-mediated responses, when increasing the rate of stimulation. Disentangling the contributions of the improved membrane expression and the slightly higher fraction of Chronos-positive SGNs with Chronos-ES/TS is challenging. Regardless of the precise contributions, these experiments suggest that Chronos-ES/TS will be a valuable tool for optogenetic applications requiring good temporal fidelity. Moreover, avoiding the largely intracellular localization of Chronos lowers the proteostatic stress to the cell.

\subsubsection{Ultrafast Chronos-mediated stimulation of the auditory pathway}

Stimulation of the auditory pathway is a prime example for an application of optogenetics requiring both high speed and light sensitivity. Upon sound stimulation, SGNs fire at hundreds of $\mathrm{Hz}$ and show sub-millisecond temporal precision of spiking relative to the stimulus. When considering optogenetics for improved cochlear prosthetics, each stimulus should not exceed the energy requirements of electrical cochlear implants which are in the range of $0.2 \mu \mathrm{J}$ per pulse (Zierhofer et al., 1995). However, so far, the threshold for optogenetic activation of SGNs reported for $\mathrm{ChR} 2$ and $\mathrm{ChR} 2$ variant $\mathrm{CatCh}$ amounted to approximately $2 \mu \mathrm{J}$, and the responses broke down for stimulation rates exceeding $20 \mathrm{~Hz}$ (Hernandez et al., 2014a).

Chronos, featuring a sub-millisecond deactivation time constant at physiological temperature (Figure 3.1) is an obvious candidate for cochlear optogenetics. So far, we had employed transuterine AAV2/6-injections into the embryonic otocyst and observed a strong basoapical gradient of expression in the cochlea (Hernandez et al., 2014a). Using AAV-PHP.B, a novel AAV vector (Deverman et al., 2016) at high titers $\left(1-2.510^{12} \mathrm{GC} / \mathrm{ml}\right)$ for injections into the cochlea of P5-P7 mice, we found highly efficient transduction of SGNs across all cochlear turns (Figure 3.5). The light thresholds estimated for oABRs mediated by postnatal AAV-PHP.B injections of Chronos-ES/TS in SGNs amounted to $7 \mu \mathrm{J}$ (14 $\mu \mathrm{J}$ for Chronos), which is substantially higher than the energy per pulse for suprathreshold stumulation in eCIs. Therefore, further improvements of membrane expression of Chronos or the design of fast channels with larger pore remain an important objective. At present, we can only speculate how much light will be required for an auditory percept mediated by Chronos-based cochlear optogenetics. Future studies using behavioral experiments will be required to address this point. Nonetheless, we argue that future optogenetic CIs might not need the high stimulation rates employed in eCIs 
(800 Hz or higher) which might help balancing a greater energy requirement per pulse of an oCI. In most animals, oABR increased in amplitude when increasing light intensity over more than one order of magnitude (Figure 3.6). Hence, the output dynamic range of optical stimulation assessed as $\mathrm{P}_{1}-\mathrm{N}_{1}$ amplitude, on average, was $>10 \mathrm{~dB}(\mathrm{~mW})$ compared to typically $<10 \mathrm{~dB}$ for coding with eCI (Zeng et al., 2008). This likely reflects the lower spread of excitation with optical stimulation as well as differences in the levels of Chronos-ES/TS expression among SGNs at the same tonotopic place of stimulation.

The temporal fidelity of Chronos-ES/TS-mediated optogenetic stimulation was estimated at the single SGN and the SGN population levels. The minimal oABR latency was considerably shorter $(1.01 \pm 0.09 \mathrm{~ms})$ than that of the first light-evoked potential, typically a trough, that we had previously reported for transgenic ChR2 mice (3.14 $\pm 0.26 \mathrm{~ms}$ (Hernandez et al., 2014a)). Moreover, the minimal duration of the light pulse required for eliciting an oABR at high light intensity was shorter $(<100 \mu \mathrm{s})$ than that found before with ChR2 or CatCh $(\geq 200 \mu \mathrm{s}$; (Hernandez et al., 2014a)). Most importantly, population responses mediated by Chronos-ES/TS followed pulse rates up to at least $1000 \mathrm{~Hz}$ unlike for $\mathrm{ChR} 2$, where oABRs were lost below $100 \mathrm{~Hz}$. Obviously, the analysis had to be restricted to a very short measurement time window in order to track the response to such high rates and hence signal propagation along the pathway cannot be demonstrated. This ABR analysis suggests that the Chronos-ES/TS mediated optogenetic stimulation achieves a temporal fidelity similar to that of acoustic coding. Using juxtacellular recordings we could demonstrate firing of single SGNs in response to trains of light pulses at hundreds of $\mathrm{Hz}$ with sub-millisecond temporal precision. Such temporal fidelity of optogenetic control of SGN firing marks a major breakthrough on the way towards using optogenetic stimulation for auditory research and for the future development of the clinical optical cochlear implant.

\subsection{Materials and Methods}

\section{Cloning}

For the cloning of pAAV_hSyn_Chronos-ES/TS as a starting material we have used pAAVEfla-DIO-eNpHR 3.0-EYFP (Addgene, plasmid nr. \#26966). In order to obtain flanked EYFP with ES/TS sequences we performed a classical PCR reaction. The primers that we used for this cloning were 5'-GAGAACCGGTCAAGAGCAGGATCAC-3' and 5'GTGGGGTACCCCTTACACCTCGTTCTC-3'. In the second step, the obtained PCR 
fragment was digested with AgeI/Acc65I (Thermo Scientific, MA, USA) (Acc65I produces compatible cohesive ends as BsrGI) gel extracted (GeneJET Gel Extraction Kit, Thermo Scientific, MA, USA) and further used for ligation. At the same time, the plasmid pAAV_hSyn_Chronos-GFP (Addgene, plasmid no. 59170) was also digested using restriction enzymes AgeI/BsrGI and used as a backbone plasmid. All obtained ligation products were further tested by the means of colony PCR and finally sequenced by an external company.

\section{Cell Culture and HEK-293T transfection}

HEK-293T cells (ATCC, USA) were cultured at $37^{\circ} \mathrm{C}$ and $5 \% \mathrm{CO}_{2}$ in DMEM (Gibco, USA) supplemented with $10 \%$ fetal calf serum (Gibco, Germany), and $1 \%$ penicillin/streptomycin (Sigma, Germany). One day prior to transient transfections the HEK293T cells were seeded on 24-well plates. The day after seeding, cells were transiently transfected with pAAV_hSyn_Chronos-ES/TS or pAAV_hSyn_Chronos using PEI 25.000MW (Polysciences Inc. USA). Two days after transfection cells were briefly washed and then fixed with $4 \%$ PFA for $10 \mathrm{~min}$. After fixation cells were mounted with Mowiol (Sigma, Germany) and processed to confocal/STED imaging. Cells were regularly tested for mycoplasma contamination. No method of cell line authentication was used.

For characterization of gating kinetics, HEK-293T cells were transfected with 3-5 $\mu \mathrm{g}$ of plasmid DNA encoding a ChR2-YFP or a Chronos-GFP fusion protein via nucleofection using a Lonza nucleofector device and the Amaxa Cell Line Nucleofection Kit V (Lonza, Switzerland; program Q-01), following the manufacturer's instructions. The pcDNA 3.1-ChR2-YFP construct was kindly provided by Ernst Bamberg (MPI for Biophysics, Frankfurt, Germany), and Chronos-GFP was kindly provided by Edward Boyden (MIT, Cambridge, MA). After transfection, the cells were plated on poly-L-lysine coated $10 \mathrm{~mm}$ glass cover slips. Electrophysiological characterization was performed 20 to $30 \mathrm{~h}$ after transfection.

\section{Electrophysiology}

Transfection success was accessed via fluorescence in an inverted Axiovert 135 TV fluorescence microscope (ZEISS, Germany), equipped with a 40X/0.65 N.A. Achroplan objective. Only cells isolated from others and exhibiting clear membrane fluorescence signal 
were recorded. Whole-cell patch-clamp recordings were made in voltage-clamp mode (-60 $\mathrm{mV}$ holding potential) using an EPC 10 USB amplifier (HEKA Elektronik, Germany). Current signals were low-pass filtered at $3 \mathrm{kHz}$ and digitized at $20 \mathrm{kHz}$. Patch-pipettes were prepared from PG10165-4 glass capillaries (World Precision Instruments, USA) in a PIP 6 vertical puller (HEKA Elektronik, Germany), and had resistances between 3-5 $\mathrm{M} \Omega$ when filled with the following pipette solution (in $\mathrm{mM}$ ): $110 \mathrm{NaCl}, 10 \mathrm{Na}_{4}$-EGTA, $4 \mathrm{MgCl}_{2}, 10 \mathrm{HEPES}$, and 10 Glucose (pH 7.4 and osmolarity between 285-290 mOsm). The bath solution contained (in $\mathrm{mM}$ ): $145 \mathrm{NaCl}, 3 \mathrm{KCl}, 1 \mathrm{MgCl}_{2}, 2 \mathrm{CaCl}_{2}, 10 \mathrm{HEPES}$, and 15 Glucose (osmolarity between 310-315 mOsm and $\mathrm{pH} 7.35$ ). Series resistance (always < $20 \mathrm{M} \Omega$ before compensation) was electronically compensated 60 to $90 \%$. For recordings at physiological temperature, warm solution was perfused by gravitation in the recording chamber via an HPT-2 in-line heater (ALA Scientific Instruments, USA) controlled by a TC-10 temperature controller (NPI Electronic, Germany). Temperature at the recording chamber was monitored via a thermistor placed in the chamber and it was maintained at $36 \pm 1{ }^{\circ} \mathrm{C}$. Light-stimulation was achieved using a $480 \mathrm{~nm}$ diode (5 W Luxeon rebel color with Lambertian dome; Philips Lumileds) controlled by a custom-built controller. Stimulation protocols consisted of 1 s-long light steps at different intensities $\left(0.08,0.14,0.19,0.23,0.27 \mathrm{~mW} \mathrm{~mm}^{-2}\right)$, with a $11 \mathrm{~s}$-long dark period in between, or a 10 s-long light chirp linearly-increasing from 0.1 to 100 (or 500) $\mathrm{Hz}$ and maximum amplitude of $0.27 \mathrm{~mW} \mathrm{~mm}^{-2}$ (following a $1 \mathrm{~s}$-long pre-pulse at half maximum intensity). The LED output faithfully followed the voltage command up to at least $5 \mathrm{kHz}$. Light-elicited currents were analyzed offline in Matlab 2011b/2014b (Mathworks, USA), Igor Pro 6/7 (Wavemetrics, USA) and OriginPro 7 (OriginLab, USA), and this included the correction of baseline drifts during the 10 s-long chirp recordings. For recordings at $36^{\circ} \mathrm{C}$ occasionally spike-like artifacts occurred due to the in-line heater. Those spikes were removed for display purposes in Figure 3.1.

\section{Dissociation and culture of hippocampal neurons, AAV infection and immunocytochemistry}

E18 pregnant Wistar rats were sacrificed using $\mathrm{CO}_{2}$, embryos were removed and heads of embryos were placed in a $10 \mathrm{~cm}$ petri dish containing ice-cold dissection media (HBSS (Gibco) + $10 \mathrm{mM}$ Hepes (Gibco)). Brains were removed and collected in fresh dissection medium. Hippocampi were separated from the brain and meninges were removed. Hippocampi were digested with $2 \mathrm{ml}$ pre-warmed $37^{\circ} \mathrm{C} 0.05 \%$ trypsin-EDTA (Gibco, Germany) for $20 \mathrm{~min}$ at 
$37^{\circ} \mathrm{C}$. Trypsin was removed and the tissue was washed three times with $4^{\circ} \mathrm{C}$ dissection medium. Dissection medium was replaced with $1 \mathrm{ml}$ pre-warmed NB+ (Neurobasal with 1X B-27 supplement, 1X Glutamax and penicillin (5000 $\left.\mathrm{U} \mathrm{ml}^{-1}\right) /$ streptomycin $\left(5000 \mu \mathrm{g} \mathrm{ml}^{-1}\right)$; all from Gibco) and tissue was triturated by gentle pipetting. The tissue suspension was filtered through a $100 \mathrm{~mm}$ cell strainer (BD Biosciences). Cells were counted using the trypan blue exclusion method and cultured on $12 \mathrm{~mm}$ glass coverslips (Thermo Scientific) coated with poly-D-lysine (PDL, Sigma) dissolved in 0.1 M borate buffer, in 24-well plates (CytoOne) at a density of 80000 hippocampal neurons per $\mathrm{cm}^{2}$ in $\mathrm{NB}^{+}$medium in a HeraCell 240i cell culture incubator (Thermo Scientific) at $37^{\circ} \mathrm{C}$ and $5 \% \mathrm{CO}_{2}$. On DIV 10, neurons were infected with $1 \mu \mathrm{AAV} 2 / 6$ Chronos, AAV2/6 Chronos-ES/TS or the same constructs packed in PHP.B virus capsid. $72 \mathrm{~h}$ after infection, neurons were briefly washed with pre-warmed PBS and then fixed with $4 \%$ PFA at RT for $10 \mathrm{~min}$. After fixation and several steps of washing, neurons were subjected to immunocytochemistry (rabbit anti-Map2 primary antibody, 1:1000, Abcam and goat anti-rabbit Alexa 555 secondary antibody, 1:1000, Invitrogen) and later on to confocal microscopy (Leica SP5).

\section{Virus purification}

AAVs were generated in HEK-293T cells (ATCC) using polyethylenimine transfection (25.000 MW, Polysciences, USA) (Gray et al., 2011; Deverman et al., 2016). In brief, triple transfection of HEK-293T cells was performed using pHelper plasmid (TaKaRa/Clontech), trans-plasmid providing viral capsid PHP.B (generous gift from Ben Deverman and Viviana Gradinaru, Caltech, USA) and cis-plasmid providing Chronos or Chronos-ES/TS (Figure 3.2A). The cell line was regularly tested for mycoplasma. We harvested viral particles $72 \mathrm{~h}$ after transfection from the medium and $120 \mathrm{~h}$ after transfection from cells and the medium. Viral particles from the medium were precipitated with $40 \%$ polyethylene glycol 8000 (Acros Organics, Germany) in $500 \mathrm{mM} \mathrm{NaCl}$ for $2 \mathrm{~h}$ at $4^{\circ} \mathrm{C}$ and then after centrifugation at $4000 \mathrm{~g}$ for 30 min combined with cell pellets for processing. The cell pellets were suspended in $500 \mathrm{mM}$ $\mathrm{NaCl}, 40 \mathrm{mM}$ Tris, $2.5 \mathrm{mM} \mathrm{MgCl} 2, \mathrm{pH} 8$, and $100 \mathrm{U} \mathrm{mL}^{-1}$ of salt-activated nuclease (Arcticzymes, USA) at $37^{\circ} \mathrm{C}$ for $30 \mathrm{~min}$. Afterwards, the cell lysates were clarified by centrifugation at $2000 \mathrm{~g}$ for $10 \mathrm{~min}$ and then purified over iodixanol (Optiprep, Axis Shield, Norway) step gradients (15\%, $25 \%, 40 \%$ and $60 \%$ ) (Zolotukhin et al., 1999; Grieger et al., 2006) at $320,006 \mathrm{~g}$ for $2.25 \mathrm{~h}$. Viruses were concentrated using Amicon filters (EMD, 
UFC910024) and formulated in sterile phosphate-buffered saline (PBS) supplemented with $0.001 \%$ Pluronic F-68 (Gibco, Germany). Virus titers were measured using AAV titration kit (TaKaRa/Clontech) according to manufacturer's instructions by determining the number of DNase I resistant vg using qPCR (StepOne, Applied Biosystems). Purity of produced viruses was routinely checked by silver staining (Pierce, Germany) after gel electrophoresis (Novex ${ }^{\mathrm{TM}}$ 4-12 \% Tris-Glycine, Thermo Fisher Scientific) according to manufacturer's instructions. The presence of viral capsid proteins was positively confirmed in all virus preparations. Viral stocks were kept at $-80{ }^{\circ} \mathrm{C}$ until the experimental day.

\section{Transuterine AAV injection into the cochlea}

For in vivo transduction, anesthesia was induced with a mixture of ketamine and xylazine $\left(0.125 / 5 \mathrm{mg} \mathrm{kg}^{-1}\right)$ and maintained with isoflurane $(1 \%-2 \%)$. Viral inoculum $(\sim 250 \mathrm{nl}, 4 \mathrm{e}+8$ particles $\mu^{-1}$ ) was microinjected through the uterus into the mouse otocyst from E11.5 to E12.5 as previously described (Brigande et al., 2009; Reisinger et al., 2011). Only the left otocyst of each embryo was injected. The non-injected contralateral ear served as an internal control. AAV2/6_hSyn_Chronos-GFP and AAV2/6_hSyn_Chronos-ES/TS were purchased from the University of North Carolina Vector Core, Chapel Hill, USA.

\section{Postnatal AAV injection into the cochlea}

Postnatal AAV-injection into scala tympani of the left ear via the round window was performed in P5-P7 wild-type C57BL/6 mice essentially as described (Akil et al., 2012) using AAV-PHP.B viral capsids and hSyn promoter to drive transgenic expression of opsins in SGNs. In brief, under general isoflurane anaesthesia and local analgesia achieved by means of xylocaine, the left ear was approached via a dorsal incision and the round window membrane was identified and gently punctured using a borosilicate capillary pipette that was kept in place to inject approximately $1-1.5 \mu \mathrm{l}$ of AAV2/6_hSyn-Chronos $\left(2.33 \mathrm{E}+12 \mathrm{GC} \mathrm{ml}^{-1}\right)$, AAV2/6_hSynChronos-ES/TS $\left(2.2 \mathrm{E}+12 \mathrm{GC} \mathrm{ml}^{-1}\right)$, PHP.B_hSyn-Chronos-GFP $\left(1.07 \mathrm{E}+12 \mathrm{GC} \mathrm{ml}^{-1}\right)$ or PHP.B_hSyn-Chronos-ES/TS-EYFP (2.31E+12 $\left.\mathrm{GC} \mathrm{ml}^{-1}\right)$. After virus application, the tissue above the injection site was repositioned and the wound was sutured and buprenorphine $\left(0.1 \mathrm{mg} \mathrm{kg}^{-1}\right)$ was applied as pain reliever. Recovery of the animals was then daily tracked. In 
all experiments, mice were randomly selected for injection. No blinding was possible since injections had to be performed in the left ear leaving the right ear as an internal control. Hence, surgery prior to stimulation needed to be done in the injected ear. Animals were then kept in a $12 \mathrm{~h}$ light/dark cycle, with access to food and water ad libitum. All experiments were done in compliance with the national animal care guidelines and were approved by the board for animal welfare of the University Medical Center Goettingen and the animal welfare office of the state of Lower Saxony. The calculation of animal number was performed prior to starting experiments. We planned to use the Wilcoxon Rank Sum Test and an error probability alpha smaller than 0.05 , a power (1-beta) of 0.95 and effect size depending on the precise experimental protocol.

\section{Immunostaining and imaging of cochlear cryosections}

Cochleae were fixed with $4 \%$ paraformaldehyde in phosphate buffered saline $(1 \mathrm{~h})$. Sections of the cochlea were cryosectioned following 0.12 M EDTA decalcification. After incubation of sections for $1 \mathrm{~h}$ in goat serum dilution buffer (16\% normal goat serum, $450 \mathrm{mM} \mathrm{NaCl}, 0.6 \%$ Triton X-100, $20 \mathrm{mM}$ phosphate buffer, $\mathrm{pH}$ 7.4) primary antibodies were applied over night at $4^{\circ} \mathrm{C}$. The following antibodies were used: chicken anti-GFP (catalog no.: ab13970, Abcam, 1:500), guinea pig anti-parvalbumin (catalog no.: 195004, Synaptic Systems, 1:300). Secondary AlexaFluor-labeled antibodies (goat anti-chicken 488 IgG (H+L), catalog no.: A-11039, Thermo-Fisher Scientific, 1:200; goat-anti guinea pig 568 IgG (H+L), catalog no.: A1107, Thermo-Fisher Scientific, 1:200) were applied for $1 \mathrm{~h}$ at room temperature. Confocal images were collected using a SP5 microscope (Leica) and processed in ImageJ. Expression was considered positive when FP fluorescence in a given cell (marked by parvalbumin) was found to be higher than $3 \mathrm{SD}$ above the background fluorescence of the tissue.

For FP localization analysis line profiles (length: $7.5 \mu \mathrm{m}, 7.5 \mu \mathrm{m}$ and $1.5 \mu \mathrm{m}$ for SGNs, HEK cells and hippocampal neurons, respectively, width: 3 pixels) were centered to the outer edge of the estimated cell membrane. The line profiles were oriented perpendicular to the cell edge. Sample size was 1, 1 and 3 per cell for SGNs, HEK cells and hippocampal neurons, respectively. For membrane/intracellular expression ratio, a maximum peak detection was performed for membranous area (defined positionally on the line profiles as -1 to $1 \mu \mathrm{m},-1$ to $1 \mu \mathrm{m}$ and -0.5 to $0.3 \mu \mathrm{m}$ for SGNs, HEK cells, and hippocampal neurons, respectively) and for intracellular area 
(defined positionally on the line profiles as 1.1 to $2 \mu \mathrm{m}, 1.1$ to $2 \mu \mathrm{m}$, and 0.4 to $0.5 \mu \mathrm{m}$ for SGNs, HEK cells, and hippocampal neurons, respectively).

\section{STED microscopy}

Images were acquired using an Abberior Instruments Expert Line STED microscope, with excitation lasers at $488 \mathrm{~nm}$ and STED lasers at $595 \mathrm{~nm}, 1 \mathrm{~W}$ using a $1.4 \mathrm{NA}$ 100x oil immersion objective, either in confocal or in 2D-STED mode.

\section{Optical stimulation in vivo}

The left bulla was reached using a retroauricular approach and opened to expose the cochlea. A 50/200 $\mu \mathrm{m}$ optical fiber coupled to a $594 \mathrm{~nm}$ laser (OBIS LS OPSL, $100 \mathrm{~mW}$, Coherent Inc.) was inserted into the cochlea via the round window. Irradiance was calibrated with a laser power meter (LaserCheck; Coherent Inc.).

\section{Auditory brainstem responses}

For stimulus generation and presentation, data acquisition, and off-line analysis, we used a NI System and custom-written Matlab software (The MathWorks, Inc.). Optically-evoked ABRs (oABRs) and acoustically-evoked ABRs (aABRs) were recorded by needle electrodes underneath the pinna, on the vertex, and on the back near the legs. The difference potential between vertex and mastoid subdermal needles was amplified using a custom-designed amplifier, sampled at a rate of $50 \mathrm{kHz}$ for $20 \mathrm{~ms}$, filtered $(300-3000 \mathrm{~Hz})$ and averaged across 1000 and 500 presentations (for oABRs and aABRs, respectively). The first ABR wave was detected semi-automatically with a custom-written Matlab script in which the wave was detected for each trace in a temporal window defined by the user and for which the amplitude was bigger than the average +2 standard deviations. Thresholds were determined by visual inspection as the minimum sound or light intensity that elicited a reproducible response waveform in the recorded traces. 


\section{Juxtacellular recordings from single putative SGNs}

For auditory nerve recordings, glass microelectrodes $(\sim 50 \mathrm{M} \Omega)$ were advanced through the posterior end of the anteroventral cochlear nucleus using an Inchworm micro-positioner (EXFO Burleigh, NY, USA) and aimed towards the internal auditory canal. Action potentials were amplified using an ELC-03XS amplifier (NPI Electronic, Tamm, Germany), filtered (300$3000 \mathrm{~Hz}$ ), digitized (National Instruments card PCIe-6323), analyzed and prepared for display using using custom-written Matlab (The MathWorks, Inc.) software.

When light-responsive fibers were found, $400 \mathrm{~ms}$-long pulse trains at repetition rates 20-1000 $\mathrm{Hz}$ were presented, leaving $100 \mathrm{~ms}$ inter-train recovery over 20 iterations for each tested rate. Different rates were tested following no particular order, being $20 \mathrm{~Hz}$ the first repetition rate presented across all units. For repetition rates higher or equal to $200 \mathrm{~Hz}$, parameters were computed if the spike probability was equal or superior to $5 \%$. If not, values were set to 0 . Phase-locking was quantified using the vector strength (Goldberg \& Brown, 1969), considering a cycle starting at the onset of a light pulse and ending at the onset of the subsequent pulse, and conforming to the equation: vector strength $=\frac{\sqrt{\left[\sum_{\mathrm{i}=1}^{\mathrm{n}} \cos \theta_{\mathrm{i}}\right]^{2}+\left[\sum_{\mathrm{i}=1}^{\mathrm{n}} \sin \theta_{\mathrm{i}}\right]^{2}}}{\mathrm{n}}$, being $\Theta_{1}, \Theta_{2}, \ldots, \Theta_{n}$ cycle phases in which spikes occurred. The Rayleigh test was used to evaluate the significance of vector strength: if $\mathrm{L}>13.8$, the null hypothesis is rejected at the 0.001 significance level (Hillery and Narins, 1987) and insignificant VS were set to 0 . The spike probability was calculated as the ratio between the number of spikes and the number of light-pulses. The temporal jitter is the standard deviation of spike latency across trials. The hazard function (for the temporal jitter analysis) was calculated for each stimulation rate by simulating spiking as a Poisson process at given rates (from 10 to 1000 spikes s ${ }^{-1}$ ).

\section{Data analysis}

The data were analysed using Matlab (Mathworks), Excel (Microsoft), Igor Pro (Wavemetrics), FIJI (ImageJ2), Origin (Microcal Software), and GraphPad Prism (GraphPad Software). Averages were expressed as mean \pm s.e.m. or mean \pm s.d., as specified. References to data in the main text were expressed as mean \pm s.e.m. For statistical comparison between two groups, data sets were tested for normal distribution (the D'Agostino \& Pearson omnibus normality test or the Shapiro-Wilk test) and equality of variances (F-test) followed by two-tailed unpaired 
Student's t-test, or the unpaired two-tailed Mann-Whitney U test when data were not normally distributed and/or variance was unequal between samples.

For evaluation of multiple groups, statistical significance was calculated by using one-way ANOVA test followed by Tukey's test for normally distributed data (equality of variances tested with the Brown-Forsythe test) or one-way Kruskal-Wallis test followed by Dunn's test for nonnormally distributed data.

Acknowledgments: We would like to thank Dr. Edward Boyden for providing the ChronosEGFP construct and Rachel Care for contributing in the initial phase of the project. We thank Andrea Koch, Daniela Gerke, Sandra Gerke and Christiane Senger-Freitag for expert technical assistance and Gerhard Hoch for devising hard- and software for system physiology and Jakob Neef for the help with STED microscopy. We thank Camin Dean for providing hippocampal neuronal culture. We thank Blanche Schwappach for feedback on the manuscript.

Funding: This work was funded by the European Research Council through the Advanced Grant 'OptoHear" to T.M. under the European Union's Horizon 2020 Research and Innovation program (grant agreement No. 670759) and was further supported by the German Ministry of Research and Education through the Bernstein Center for Computational Neuroscience to A.N., F.W. and T.M. (under grant number 01GQ1005A) and the grant Optical CI (under grant number 13N13729) to T.M.) and the German Research Foundation (through the DFG-Research Center (FZT103) and Cluster of Excellence (EXC171) Center for Nanoscale Microscopy and Molecular Physiology of the Brain to T.M., as well as the Leibniz Program to T.M.).

Author contributions: D.K., V.R., F.W., A.N. and T.M. designed the study. D.K. performed o/aABR recordings, immunohistochemistry and FP expression analysis. A.G., T.D. and C.W. performed initial o/aABR recordings. R.M. performed patch-clamp recordings, B.B. performed immunohistochemistry. S.S. and D.L.M. performed initial cloning. V.R. performed AAV injections, HEK293T and hippocampal neuron experiments, confocal and STED microscopy, cloning and production of the viruses. D.L.M. performed recordings from single SGNs and oABRs. A.T.H. developed analysis toolboxes for ABRs and recordings from single SGNs and performed analysis of electrophysiological data. All authors analyzed data and contributed to the writing of the manuscript. T.M., D.K., V.R. and A.N. took a lead on preparing the manuscript.

Competing interests: The authors declare no competing interests. 


\section{Expanded View Figures}

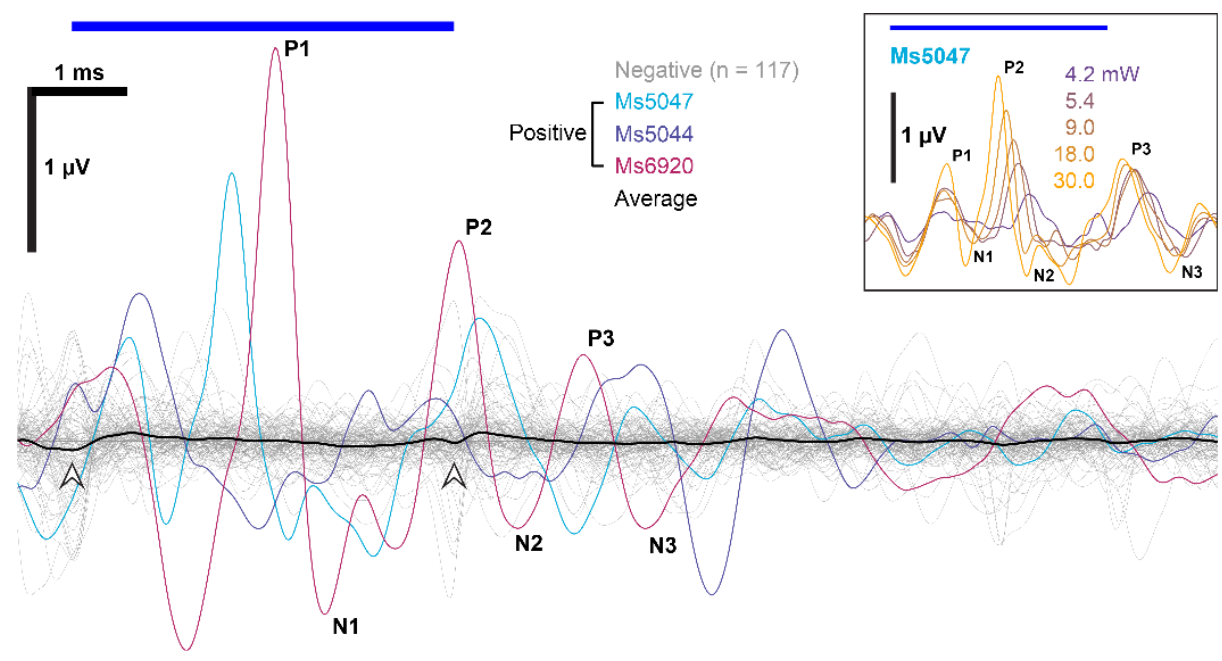

Figure EV1: Demonstrating functional expression of Chronos in SGNs following transuterine AAV2/6 injection reported by recordings of oABRs

In 3 out of dozens of animals we could record oABRs using fiber-coupled laser stimulation 412 weeks after embryonic transuterine injection of AAV2/6 carrying Chronos using 4 ms pulse trains at $10 \mathrm{~Hz}$ with $30 \mathrm{~mW}$ radiant flux. Responses were verified as oABRs by the increasing amplitude with stronger light pulses (inset: shows oABRs of an exemplary oABR-positive mouse for varying radiant flux, colors code the radiant flux in $\mathrm{mW}$ ). A small onset and offset artifact (arrowheads) is visible for the average (black) of the negative animals (gray) which we occasionally observed in the early phase of the project. 
A
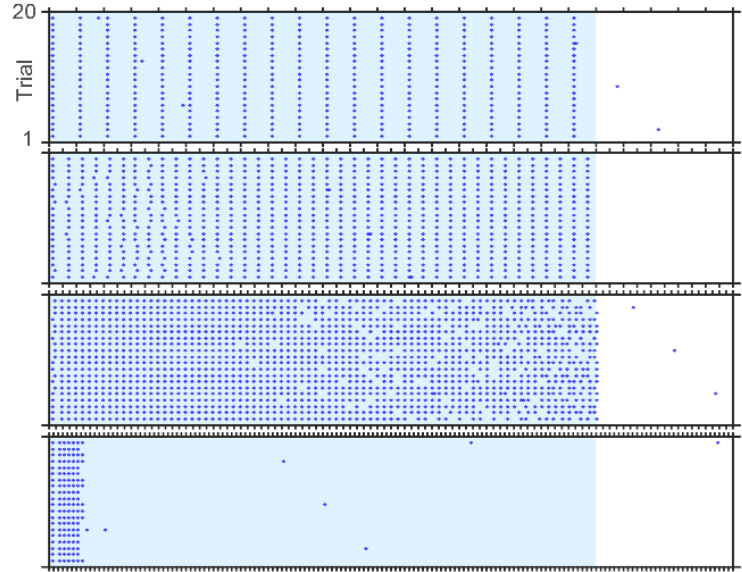

$=$
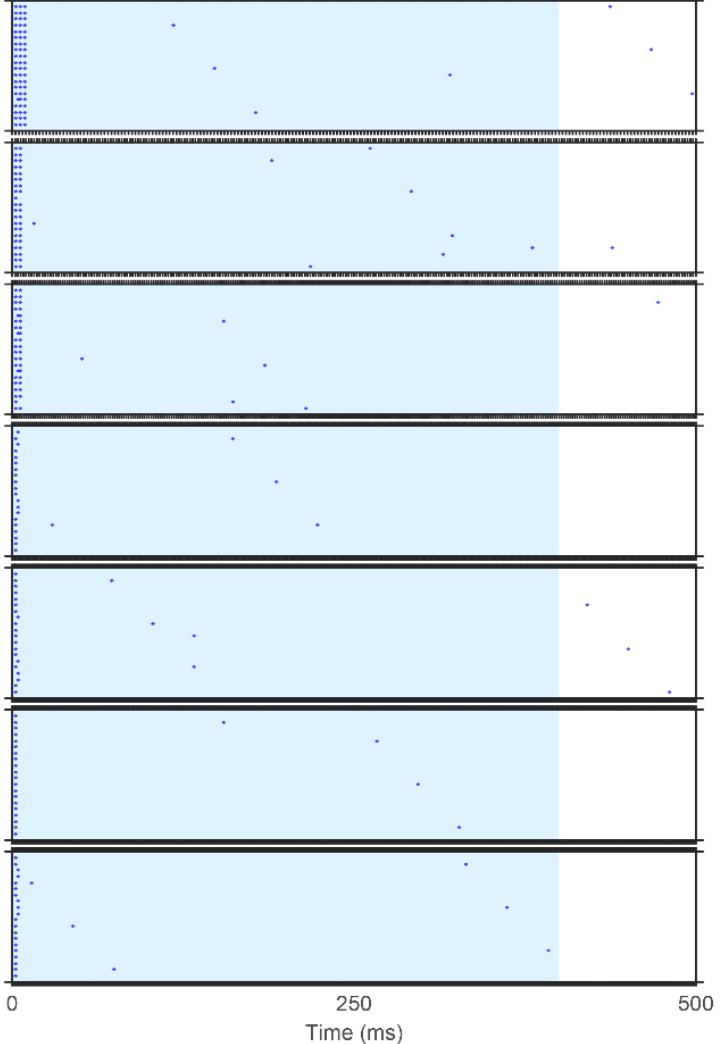

B

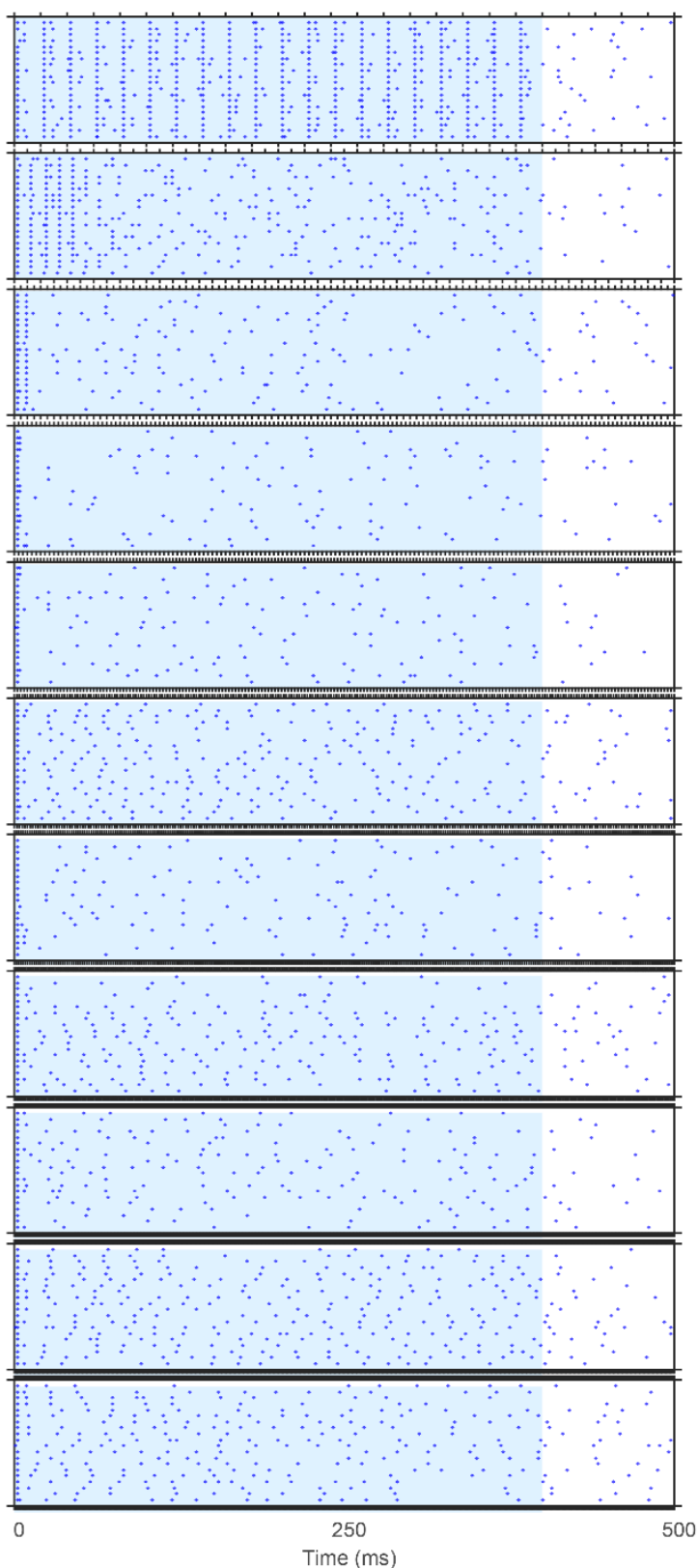

Figure EV2: Activity of two exemplary putative SGNs upon light stimulation at increasing repetition rates

A: Raster plot showing spike timing (blue symbols) of a rapidly adapting putative SGN in response to $400 \mathrm{~ms}$ pulse trains at stimulation rates $50-1000 \mathrm{~Hz}(30 \mathrm{~mW}, 1 \mathrm{~ms}$ for $50-600 \mathrm{~Hz}$; $30 \mathrm{~mW}, 0.5 \mathrm{~ms}$ for $700-1000 \mathrm{~Hz}$ ). B: Raster plot showing activity of a slowly adapting putative $\mathrm{SGN}$ in response to the stimulation paradigm described in $\mathrm{A}$. 


\section{High frequency neural spiking and auditory signaling by ultrafast red-shifted optogenetics}

Mager, T.*, Lopez de la Morena, D.*, Senn, V., Schlotte, J., D’Errico, A., Feldbauer, K., Wrobel, C., Jung, S., Bodensiek, K., Rankovic, V., Browne, L., Huet, A., Jüttner, J., Wood, P.G., Letzkus, J.J., Moser, T., Bamberg, E.

*These authors contributed equally to the work

Nat Commun. 2018; 9(1):1750

\section{https://www.nature.com/articles/s41467-018-04146-3}

doi: 10.1038/s41467-018-04146-3

Detailed author contribution of D. Lopez de la Morena:

- Experimental work and analysis:

O Initial cloning of pAAV-hSyn-f-Chrimson-EYFP (Fig.4B)

○ Immunohistochemistry, confocal imaging on cochlear cryosections, fChrimson-EYFP expression and SGN density analysis (Fig. 4D-F and Supplementary Fig. 10A-D).

$\circ$ a/oABR recordings from young and aged mice injected with AAV2/6-fChrimson-EYFP (Fig. 5 and Supplementary Fig. 10E-J)

○ In vivo recordings from single SGNs (Fig. 6 and Supplementary Fig. 11)

- Preparation of the manuscript (together with T.Ma., T.Mo., and E.B. with contributions from V.S. and J.J.L.)

Copyright notice:

This material is reprinted by permission from Springer Nature Ltd. open access journals in accordance to their copyright and licensing regulations (CC BY license 4.0 https://creativecommons.org/licenses/by/4.0/legalcode). (C) owned by the authors, 2018. 


\section{High frequency neural spiking and auditory signaling by ultrafast red-shifted optogenetics}

Authors: Thomas Mager ${ }^{1, \S}$, David Lopez de la Morena ${ }^{2, \S}$, Verena Senn ${ }^{3,4}$, Johannes Schlotte $^{1, \dagger}$, Anna D’Errico ${ }^{1}$, Katrin Feldbauer ${ }^{1, \#}$, Christian Wrobel $^{2}$, Sangyong Jung ${ }^{2, \# \#, \text { Kai }}$ Bodensiek $^{2}$, Vladan Rankovic ${ }^{2,5}$, Lorcan Browne ${ }^{2,5,7}$, Antoine Huet ${ }^{2,5}$,Josephine Jüttner ${ }^{6}$, Phillip G. Wood ${ }^{1}$, Johannes J. Letzkus ${ }^{3}$, Tobias Moser ${ }^{2,5,8} *$, Ernst Bamberg ${ }^{1}$ *

${ }^{1}$ Department of Biophysical Chemistry, Max Planck Institute of Biophysics, D-60438 Frankfurt, Germany.

${ }^{2}$ Institute for Auditory Neuroscience and InnerEarLab, University Medical Center Göttingen, D-37075 Göttingen, Germany.

${ }^{3}$ Neocortical Circuits Lab, Max Planck Institute for Brain Research, D-60438 Frankfurt, Germany.

${ }^{4}$ Ernst-Strüngmann-Institute for Neuroscience, D-60528 Frankfurt, Germany.

${ }^{5}$ Auditory Neuroscience and Optogenetics Group, German Primate Center, D-37075 Göttingen, Germany.

${ }^{6}$ Friedrich Miescher Institute for Biomedical Research, CH-4058 Basel, Switzerland.

${ }^{7}$ UCL Ear Institute, University College London, London WC1X 8EE, United Kingdom.

${ }^{8}$ Göttingen Graduate School for Neuroscience and Molecular Biosciences, University of Göttingen, D-37075 Göttingen, Germany.

Current addresses: † Biozentrum, University of Basel, CH-4056 Basel, Switzerland. \# MaxPlanck-Institut für Herz- und Lungenforschung, D-61231 Bad Nauheim, Germany. \#\# Neuro Modulation and Neuro Circuitry Group, Singapore Bioimaging Consortium (SBIC), Biomedical Sciences Institutes, A*STAR, 138667, Singapore.

$\S$ these authors contributed equally.

*Correspondence to: ernst.bamberg@biophys.mpg.de and tmoser@gwdg.de 


\subsection{Abstract}

Optogenetics revolutionizes basic research in neuroscience and cell biology and bears potential for medical applications. We develop mutants leading to a unifying concept for the construction of various channelrhodopsins with fast closing kinetics. Due to different absorption maxima these channelrhodopsins allow fast neural photoactivation over the whole range of the visible spectrum. We focus our functional analysis on the fast-switching, red-light activated Chrimson variants, because red light has lower light scattering and marginal phototoxicity in tissues. We show paradigmatically for neurons of the cerebral cortex and the auditory nerve that the fast Chrimson mutants enable neural stimulation with firing frequencies of several hundred $\mathrm{Hz}$. They drive spiking at high rates and temporal fidelity with low thresholds for stimulus intensity and duration. Optical cochlear implants restore auditory nerve activity in deaf mice. This demonstrates that the mutants facilitate neuroscience research and future medical applications such as hearing restoration.

\subsection{Introduction}

Microbial-type rhodopsins, light gated cation channels (Channelrhodopsins, ChRs) and light driven ion pumps are useful tools for multimodal optogenetic control of electrically excitable cells in culture, tissue and living animals ((Boyden et al., 2005; Nagel et al., 2005b; Zhang et al., 2007a). Since the first description of the channelrhodopsins in 2002 and 2003 a set of different channelrhodopsins including red-shifted variants like VChR1, ReaChR and Chrimson have been described (Klapoetke et al., 2014; Lin et al., 2013; Nagel et al., 2002, 2003; Zhang et al., 2008). For different purposes, ChRs were modified with respect to the kinetics, ion selectivity as well as light absorption (Gunaydin et al., 2010; Kleinlogel et al., 2011; Lin et al., 2009). ChR kinetics is a major issue, because the light sensitivity is regulated via the open lifetime of the channel (Klapper et al., 2016). Channels with a short open lifetime need correspondingly stronger light than channels with a long open lifetime for maximal photostimulation. This is due to the essential invariance of other channel parameters like single channel conductance and quantum efficiency. The mutual dependence between channel kinetics and light sensitivity accounts for the optimization of ChR expression and light delivery for successful experiments in the high frequency range. Although fast channels need stronger light 
for the activation, high speed is indispensable for many optogenetic applications in neurobiology because many types of neurons operate at high firing rates in the intact animal.

Prominent examples include spiral ganglion neurons (SGNs) of the early auditory pathway and fast spiking interneurons in cortical areas, which fire action potentials at up to several hundred $\mathrm{Hz}$ (Hu et al., 2014; Liberman, 1978). However, light stimulation of ChR2-expressing SGNs indicated a strong limitation of the temporal response fidelity (Hernandez et al., 2014b). Therefore, fast ChRs are needed and their benefit for use in auditory research has already been indicated using Chronos, a 'fast' blue light absorbing ChR, for stimulation of the cochlear nucleus (Hight et al., 2015).

Electrical cochlea implants (eCI), to date, enable speech understanding in most of approximately 500.000 otherwise deaf users. However, the bottleneck of eCI is the poor frequency resolution of coding that results from wide current spread from each electrode contact and limits speech understanding in background noise (Zeng et al., 2008). Optical cochlear implants (oCI), stimulating optogenetically modified SGNs, promise a fundamental advance of prosthetic sound coding by increasing frequency resolution, because light can be better confined than the electric field of electrodes (Hernandez et al., 2014b). For the oCI eventually to be translated into the clinic, opsins need to be delivered into the SGNs by postnatal virus-application to the ear and should endow SGNs with high light-sensitivity and temporal fidelity of spike generation, while light scattering and blue light induced phototoxicity should be minimized. Due to the aforementioned adverse effects of optogenetic stimulation using blue light; the already available, fast blue light-activated ChR variants like ChETA $\left(\tau_{\text {off }}=4.4 \mathrm{~ms}\right.$ (Gunaydin et al., 2010)) and Chronos ( $\tau_{\text {off }}=3.6 \mathrm{~ms}$ (Klapoetke et al., 2014)) have a limited applicability in animals and future clinical translation.

Here, we report that fast gating can be generally conferred to ChRs by helix 6 (helix F) mutation and demonstrate the utility of fast red-shifted ChRs for driving spiking of fast cerebral interneurons to the limit of their encoding range. Moreover, we established efficient virusmediated delivery and expression of a fast Chrimson mutant in SGNs of mice, show that singlechannel oCIs enable near-physiological spike rates and spike timing in SGNs and restore auditory activity in deaf mice. We demonstrate on several cell types in vitro and in vivo that the unfavorable low light sensitivity for activation is compensated by high expression levels of the fast Chrimson mutants. 


\subsection{Results}

\subsubsection{Fast helix F mutants and their calcium permeabilities}

Closed to open state transition is associated with movement of helix $\mathrm{F}$ in several microbial type rhodopsins (Luecke, 2001; Nakanishi et al., 2013; Subramaniam et al., 1993). Thereby helix F movement controls protonation reactions during vectorial proton transport and consequently the cycle time (Haupts et al., 1999; Vonck, 2000). Closed to open state transitions of helix F have recently been verified for ChR2 (Müller et al., 2015; Sattig et al., 2013). Motivated by these findings we performed a systematic study about the effects of helix F mutations on the closing kinetics of channelrhodopsin (Figure 4.1). We heterologously expressed ChRs helix F mutants in neuroblastoma-glioma cells (NG cells) and performed whole-cell patch-clamp experiments. The novel helix F mutant F219Y significantly accelerated the closing kinetics of ChR2 (Figure 4.1C and Table 4.1). Mutations at the homologous positions of VChR1 (F214Y), ReaChR (F259Y) and Chrimson (Y261F) also accelerated the closing kinetics (Figure 4.1B), albeit to a different extent (Figure 4.1D-F and Table 4.1). The strongest effect on the lifetime of the channels was observed in ReaChR and VChR1, where the closing kinetics is accelerated by one order of magnitude (Table 4.1).

Interestingly, the relative calcium permeability of $\mathrm{ChR} 2 \mathrm{~F} 219 \mathrm{Y} \mathrm{P}_{\mathrm{Ca}} / \mathrm{P}_{\mathrm{Na}}=0.30 \pm 0.02(\mathrm{n}=4)$ was increased compared to the relative calcium permeability of $\mathrm{ChR} 2$ wt $\mathrm{P}_{\mathrm{Ca}} / \mathrm{P}_{\mathrm{Na}}=0.13 \pm 0.01(\mathrm{n}=4)$. Permeability ratios were calculated according to the Goldman-Hodgkin-Katz equation (Hille, 2001) with the measured values of the reversal potentials after replacing external sodium by calcium. The critical role of a tyrosine at the homologous position on the calcium permeability is verified in ReaChR and Chrimson (Supplementary Table 1). Of note F219 (ChR2 numbering) points to $\mathrm{L} 132$ (ChR2 numbering) on helix $\mathrm{C}$ in the $\mathrm{C} 1 \mathrm{C} 2$ chimera crystal structure (Figure 4.1A). ChR2 L132C has an increased calcium permeability (CatCh, calcium translocating channelrhodopsin (Kleinlogel et al., 2011)). In contrast to the FY mutations on helix F, which accelerate the closing kinetics the L132C mutation (helix C) as well as the corresponding mutations at the homologous positions of VChR1, ReaChR and Chrimson significantly slowed the closing kinetics (Table 4.1). Structural information, the effect on the kinetics and the effect on the calcium permeability indicate a probable interaction of helix $\mathrm{C}$ and helix $\mathrm{F}$ at those critical residues. 

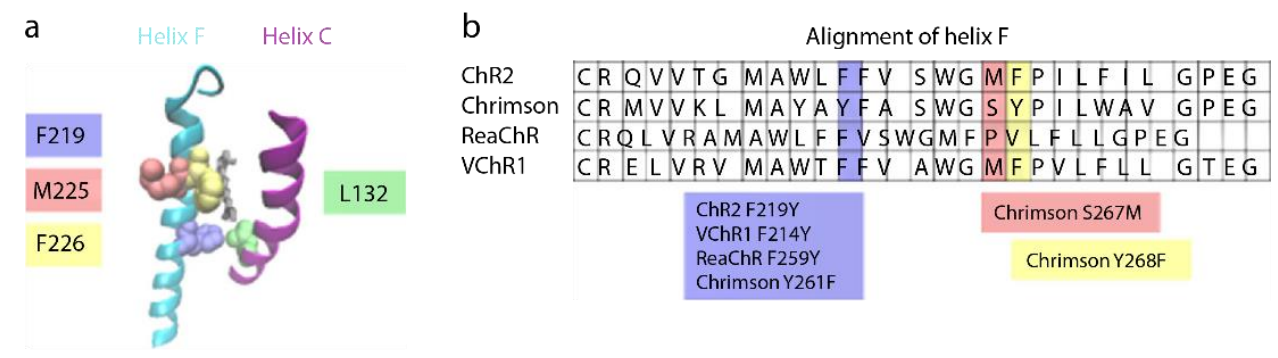

C
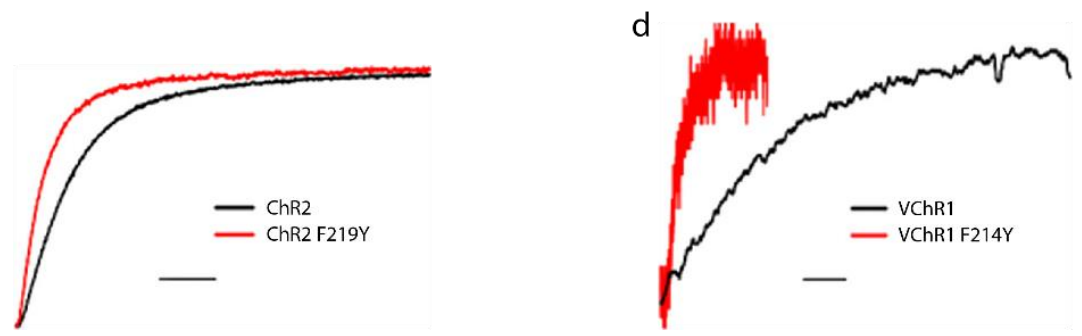

e
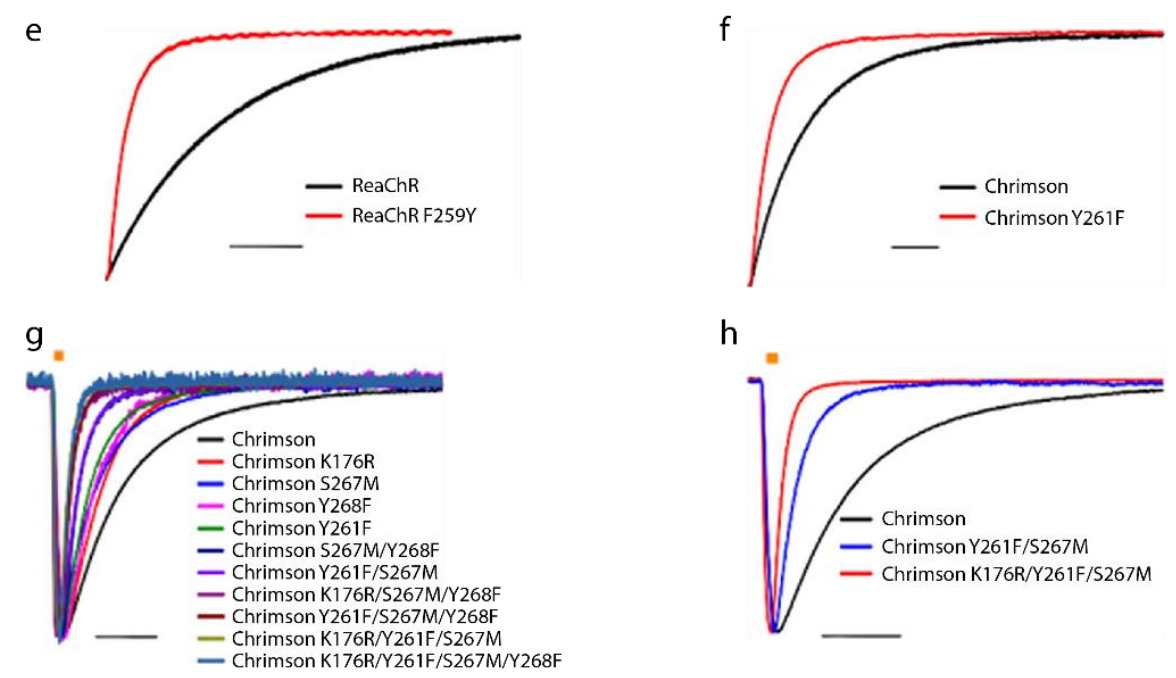

Figure 4.1: Channelrhodopsin mutants with accelerated closing kinetics

A: Helix F and helix C of channelrhodopsin (Kato et al., 2012) Residues changing the offkinetics are highlighted (ChR2 numbering). B: ClustalW alignment of the helix F of ChR2, Chrimson, ReaChR and VChR1. Colored boxes show the channelrhodopsin mutants. C-H: NG cells heterologously expressing channelrhodopsin variants were investigated by whole-cell patch-clamp experiments at a membrane potential of $-60 \mathrm{mV}$. C: Typical photocurrents of ChR2-EYFP (black trace), ChR2-EYFP F219Y (red trace); D: VChR1-EYFP (black trace), VChR1-EYFP F214Y (red trace); E: ReaChR-Citrine (black trace), ReaChR-Citrine F259Y (red trace); F: Chrimson-EYFP (black trace) and Chrimson-EYFP Y261F (red trace) immediately after cessation of $0.5 \mathrm{~s}$ illumination at a saturating light intensity of $23 \mathrm{~mW} / \mathrm{mm}^{2}$ and a wavelength of C: $\lambda=473 \mathrm{~nm}, \mathrm{D}: \lambda=532 \mathrm{~nm}, \mathrm{E}: \lambda=532 \mathrm{~nm}$ and F: $\lambda=594 \mathrm{~nm}$. G: Typical photocurrents of Chrimson-EYFP mutants, which were measured in response to $3 \mathrm{~ms}$ lightpulses $\left(23 \mathrm{~mW} / \mathrm{mm}^{2}, \lambda=594 \mathrm{~nm}\right)$. H: For clear illustration solely the photocurrents of Chrimson-EYFP, Chrimson-EYFP Y261F/S267M (f-Chrimson-EYFP) and Chrimson-EYFP K176R/Y261F/S267M (vf-Chrimson-EYFP) are shown. Photocurrents were normalized for comparison. Scale bars: C: $10 \mathrm{~ms}$, D, F: $30 \mathrm{~ms}$, E: $100 \mathrm{~ms}$, G, H $20 \mathrm{~ms}$. 
Table 4.1: Off-kinetics ( $\tau_{\text {off }}$ ) of channelrhodopsin variants

Shown are the average $\tau_{\text {off }}$ values and the corresponding s.d. NG cells transiently expressing channelrhodopsin variants were investigated by whole-cell patch-clamp experiments at $-60 \mathrm{mV}$. The $\tau_{\text {off }}$ values were determined as described in the Methods section. ChR2 (Chlamydomonas reinhardtii Channelrhodopsin 2, $\mathrm{n}=3$ ) (Nagel et al., 2003), ChR2 L132C (CatCh) (Kleinlogel et al., 2011), ReaChR (Red-activatable Channelrhodopsin, $n=3$ ) (Lin et al., 2013), VChR1 (Volvox channelrhodopsin 1, $\mathrm{n}=3$ ) (Zhang et al., 2008), Chrimson (Chlamydomonas noctigama Channelrhodopsin, $\mathrm{n}=3$ ), Chrimson K176R $($ ChrimsonR, $\mathrm{n}=5$ ) (Klapoetke et al., 2014), homologous mutants to CatCh: ReaChR L172C ( $n=3)$, VChR1 L127C $(\mathrm{n}=3)$, Chrimson L174C $(\mathrm{n}=11)$, novel mutants: Chrimson Y261F/S267M (f-Chrimson, $\mathrm{n}=4$ ), Chrimson K176R/Y261F/S267M (vf-Chrimson, $\mathrm{n}=7$ ), ChR2 F219Y ( $=4$ ), ReaChR F259Y $(n=3)$, VChR1 F214Y $(n=3)$, Chrimson Y261F $(n=7)$, Chrimson S267M $(n=5)$, Chrimson Y268F ( $n=4)$, Chrimson S267M/Y268F ( $=3$ ), Chrimson K176R/S267M/Y268F $(\mathrm{n}=3)$, Chrimson Y261F/S267M/Y268F $(\mathrm{n}=4)$, Chrimson K176R/Y261F/S267M/Y268F $(\mathrm{n}=5)^{\mathrm{a}}$ value from ChR2 L132C is taken from (Kleinlogel et al., 2011).

\begin{tabular}{|ll|}
\hline Channelrhodopsin variant & $\tau_{\text {off }}(\mathrm{ms})$ \\
\hline ChR2 & $9.5 \pm 2.8$ \\
ChR2 F219Y & $5.2 \pm 1.3$ \\
ChR2 L132C & $16 \pm 3$ a \\
ReaChR & $361.0 \pm 75.8$ \\
ReaChR F259Y & $28.8 \pm 3.8$ \\
ReaChR L172C & $3103.7 \pm 1445.2$ \\
VChR1 & $119.7 \pm 9.7$ \\
VChR1 F214Y & $12.6 \pm 1.6$ \\
VChR1 L127C & $656.4 \pm 129.8$ \\
Chrimson & $24.6 \pm 0.9$ \\
Chrimson Y261F & $9.7 \pm 1.5$ \\
Chrimson L174C & $52.8 \pm 6.0$ \\
Chrimson K176R & $12.2 \pm 0.8$ \\
Chrimson S267M & $12.1 \pm 1.5$ \\
Chrimson Y268F & $11.3 \pm 1.0$ \\
Chrimson S267M/Y268F & $6.3 \pm 1.0$ \\
Chrimson Y261F/S267M & $5.7 \pm 0.5$ \\
Chrimson K176R/S267M/Y268F & $4.9 \pm 0.5$ \\
Chrimson Y261F/S267M/Y268F & $3.5 \pm 0.5$ \\
Chrimson K176R/Y261F/S267M & $2.7 \pm 0.3$ \\
Chrimson K176R/Y261F/S267M/Y268F & $2.8 \pm 0.3$ \\
\hline
\end{tabular}

\subsubsection{Chrimson mutants with accelerated closing kinetics}

As shown above, the Y261F mutation speeds up channel closing in Chrimson (Figure 4.1F, Table 4.1). We identified two additional helix F mutations, which accelerated Chrimson's closing kinetics, namely S267M and Y268F (Figure 4.1B,G and Table 4.1). The combination of the helix F mutations had a cumulative effect, further accelerating channel closing by up to one order of magnitude (Figure $4.1 \mathrm{G}$ and Table 4.1). Chrimson mutants carrying the Y268F mutation showed a reduced expression in NG cells and a hypsochromic shift of their action 
spectra by $11 \mathrm{~nm}$ (Supplementary Figure 1 and Supplementary Table 2). The hypsochromic shift might result from an interaction of F268 with the polyene chain of the retinal, as this was shown for F265 (F226, ChR2-numbering), located at the homologous position in the C1C2 structure (Figure 4.1A).

Of special interest for optogenetic applications are the fast mutant Chrimson Y261F/S267M (fChrimson) and the very fast mutant Chrimson K176R/Y261F/S267M (vf-Chrimson), which carries the additional K176R mutation (Figure 4.1H). As described earlier (Klapoetke et al., 2014), the closing kinetics of Chrimson K176R (ChrimsonR), is accelerated by a factor of $\sim 2$ compared to wildtype (Table 4.1). The closing kinetics of f-Chrimson were strongly accelerated from $\tau_{\text {off }}=24.6 \pm 0.9 \mathrm{~ms}$ (wt-Chrimson, $\mathrm{n}=5$ ) to $\tau_{\text {off }}=5.7 \pm 0.5 \mathrm{~ms}$ (f-Chrimson, $\mathrm{n}=5$ ). At the same time f-Chrimson was highly expressed in NG cells (Supplementary Table 2). vf-Chrimson had ultrafast closing kinetics of $\tau_{\mathrm{off}}=2.7 \pm 0.3 \mathrm{~ms}(\mathrm{n}=7)$, which is at least as fast as the closing kinetics of Chronos ( $\tau_{\text {off }}=3.6 \pm 0.2 \mathrm{~ms}$ ) (Klapoetke et al., 2014), the fastest Channelrhodopsin known to date. Of note, the action spectra of f-Chrimson and vf-Chrimson were not blue-shifted (Supplementary Figure 1), thereby preserving the benefits of longer wavelength activation. Compared to Chrimson wt, the functional properties of the mutants were almost unaltered with respect to the linear voltage dependence of the photocurrents (Supplementary Figure 2), cation permeabilities (Supplementary Table 3), the moderate slowing of the closing kinetics at positive voltages (Supplementary Figure 3) and peak current inactivation (Supplementary Figure 4). Of note, the measured cation permeabilities contradict a recent publication (Vierock et al., 2017), but are in accordance with a previous study (Klapoetke et al., 2014). At a temperature of $34^{\circ} \mathrm{C}$ we measured $\tau_{\text {off }}$ values of $3.2 \pm 0.2 \mathrm{~ms}(\mathrm{n}=3)$ for f-Chrimson and $1.6 \pm 0.1 \mathrm{~ms}(\mathrm{n}=3)$ for vfChrimson (Supplementary Figure 5). Hence the ultrafast kinetics of the Chrimson mutants in principle enables neural photostimulation in an exceptionally high frequency range of up to $\sim 600 \mathrm{~Hz}$.

\subsubsection{Ultrafast red-shifted optogenetics}

We heterologously expressed f-Chrimson and vf-Chrimson in primary cultures of rat hippocampal neurons by means of adeno-associated virus mediated gene transfer (AAV2/1). Patch-clamp experiments proved a robust neuronal expression and confirmed the substantially faster kinetics of the mutants (Supplementary Table 4). The application of light pulses $\left(\lambda_{1}=594\right.$ 
$\mathrm{nm}, \lambda_{2}=640 \mathrm{~nm}$ ) triggered spiking with high reliability (Figure 4.2 and Supplementary Figure 7). The investigation of the dependence of spike probability on light pulse intensity showed that neural photostimulation via f-Chrimson $\left(0.37-1.27 \mathrm{~mW} / \mathrm{mm}^{2}\right)$ and vf-Chrimson $(0.09-3.18$ $\mathrm{mW} / \mathrm{mm}^{2}$ ) required stronger light compared to the neural photostimulation via Chrimson wt $\left(0.09-0.7 \mathrm{~mW} / \mathrm{mm}^{2}\right)$ (Figure 4.2E-G and Supplementary Table 4). That finding could be explained by the investigation of the light dependence of the Chrimson variants, which revealed significantly higher EC50 values for the fast Chrimson mutants (Supplementary Figure 6). Higher EC50 values for the fast Chrimson mutants are expected, because, as described above, light sensitivity is regulated via the open time of the channel (Klapper et al., 2016). However, due to the high expression of the fast Chrimson mutants in neurons, which is demonstrated by the large current density ( $30 \mathrm{pA} / \mathrm{pF}$ ) of their photocurrents (Supplementary Table 4) spiking with a probability of $100 \%$ could be triggered with low intensity light pulses of $0.65 \pm 0.31$ $\mathrm{mW} / \mathrm{mm}^{2}(\mathrm{n}=15)$ for $\mathrm{f}-$ Chrimson and $1.25 \pm 1.02 \mathrm{~mW} / \mathrm{mm}^{2}(\mathrm{n}=15)$ for vf-Chrimson (Supplementary Table 4). Note that potential space clamp issues might have lowered the determined current density values. The large variability of the dependence of the spike probability on the light intensity (Fig. 4.2E-G and Supplementary Table 4) is likely due to expression differences as well as the variability of membrane resistance, capacitance and spiking threshold of the investigated neurons.

The primary culture of rat hippocampal neurons comprises a multitude of different neuronal subtypes, most of which have a maximal firing frequency of 40-60 Hz (Gunaydin et al., 2010). Therefore, in most cases spike failures occurred at a frequency of $60 \mathrm{~Hz}$ (Figure 4.2C). In single cases a frequency of $100 \mathrm{~Hz}$ was achieved (Figure 4.2D). The investigation of neural photostimulation in the high frequency range is impeded by the heterogeneity of the primary neuronal culture. Therefore, we conducted patch-clamp experiments on parvalbumin-positive interneurons heterologously expressing vf-Chrimson. Parvalbumin-positive interneurons display a fast spiking phenotype, and predominantly supply inhibition to the perisomatic domain of other neurons (Hu et al., 2014). Heterologous expression of vf-Chrimson was achieved by intracerebroventricular injection of AAVs in transgenic mice that expressed tdTomato under the control of the parvalbumin promoter. Therefore, parvalbumin-positive interneurons could be identified in neocortical brain slices by their red fluorescence (Supplementary Figure 8A). 

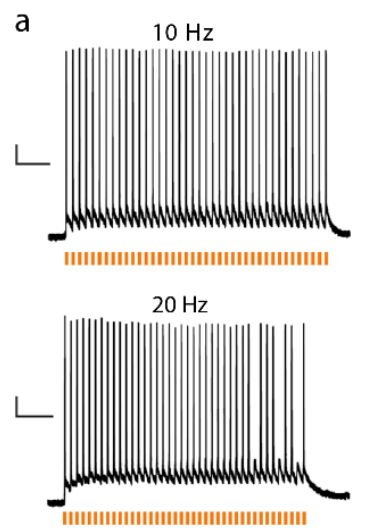

$40 \mathrm{~Hz}$

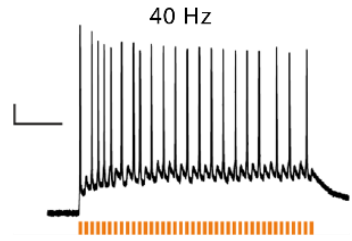

C

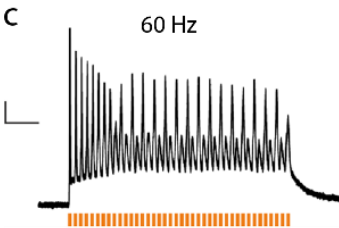

d

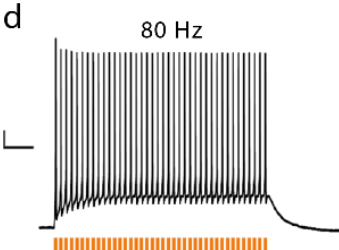

b

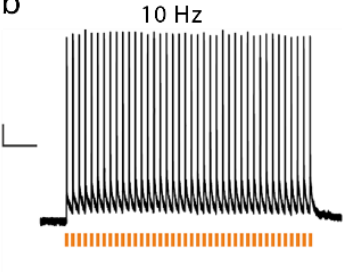

$20 \mathrm{~Hz}$

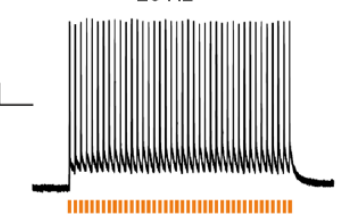

$40 \mathrm{~Hz}$

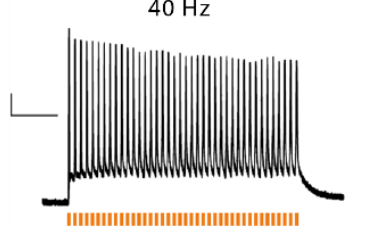

$60 \mathrm{~Hz}$
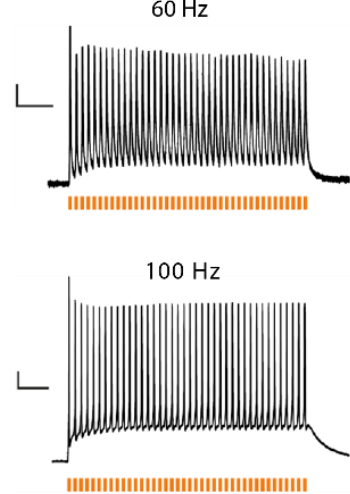
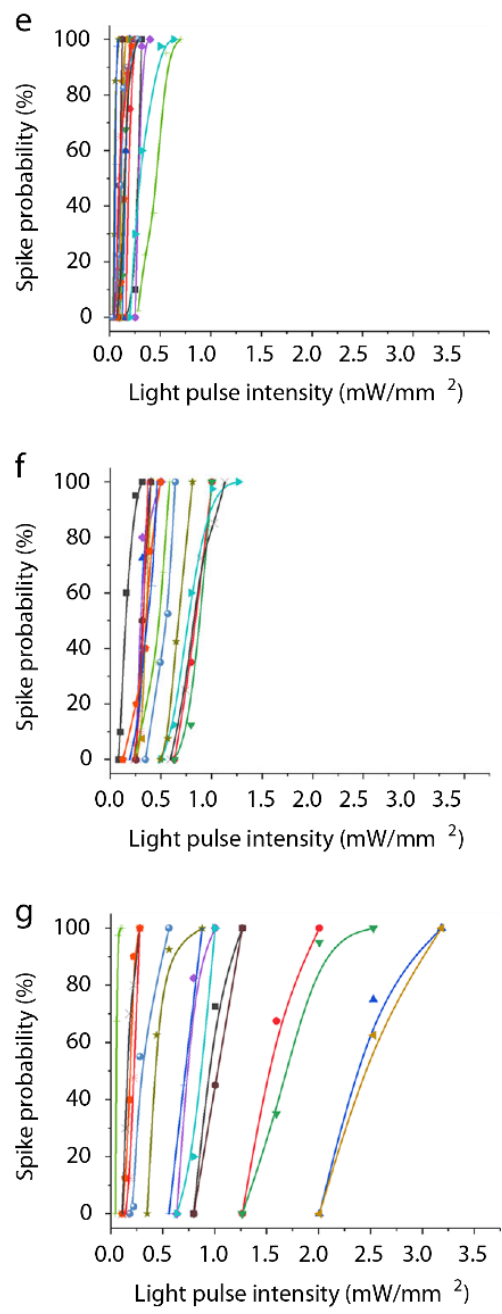

Figure 4.2: Light-induced spiking in rat hippocampal neurons

A-D: Spiking traces at different light-pulse frequencies. Rat hippocampal neurons heterologously expressing A: Chrimson-EYFP, B: Chrimson-EYFP K176R/Y261F/S267M (vfChrimson-EYFP) and C-D: Chrimson-EYFP Y261F/S267M (f-Chrimson-EYFP) were investigated by whole cell patch-clamp experiments under current-clamp conditions $\left(\lambda=594 \mathrm{~nm}\right.$, pulse width $=3 \mathrm{~ms}$, saturating intensity of $\left.11-30 \mathrm{~mW} / \mathrm{mm}^{2}\right)$. C: Traces from two different cells at a stimulation frequency of $60 \mathrm{~Hz}$. D: Traces from one cell at stimulation frequencies of $80 \mathrm{~Hz}$ and $100 \mathrm{~Hz}$. E-G: The dependence of spike probability on light pulse intensity for E: Chrimson-EYFP (15 different cells), F: Chrimson-EYFP Y261F/S267M (fChrimson-EYFP) (15 different cells), and G: Chrimson-EYFP K176R/Y261F/S267M (vfChrimson-EYFP) (15 different cells). The action potentials were triggered by 40 pulses $(\lambda=594 \mathrm{~nm}$, pulse width $=3 \mathrm{~ms}, v=10 \mathrm{~Hz})$ of indicated light intensities. In order to determine the spike probability, the number of light-triggered spikes was divided by the total number of light pulses. Scale bars: $y$-axis: $10 \mathrm{mV}$, time-axis: (A-B, $10 \mathrm{~Hz}) 500 \mathrm{~ms}(\mathrm{~A}-\mathrm{B}, 20 \mathrm{~Hz}) 300 \mathrm{~ms}$ $(\mathrm{A}-\mathrm{B}, 40 \mathrm{~Hz}) 200 \mathrm{~ms}(\mathrm{C}, 60 \mathrm{~Hz}) 100 \mathrm{~ms}(\mathrm{D}, 80 \mathrm{~Hz}) 70 \mathrm{~ms}(\mathrm{D}, 100 \mathrm{~Hz}) 50 \mathrm{~ms}$.

Using current injections we determined a maximal intrinsic firing frequency of $301 \pm 29 \mathrm{~Hz}$ $(\mathrm{n}=8)$ for the parvalbumin-positive interneurons, as expected for the fast spiking phenotype 
(Figure 4.3A,B) (Hu et al., 2014). Of note vf-Chrimson enabled neural photostimulation up to the intrinsic limit of the cells with high temporal precision (Figure 4.3C-E and Supplementary Figure 8C). As demonstrated, some cells followed photostimulation up to $400 \mathrm{~Hz}$ (Figure 4.3C,D). In 2/7 cells the occurrence of extra spikes in response to the light pulse was observed (Supplementary Figure 8B) (Gunaydin et al., 2010), which compromised the fidelity of neural photostimulation in those cases. We note that similar to previous work using AAV transduction and single photon stimulation (Klapoetke et al., 2014), it was necessary to adjust irradiation intensity individually for each neuron to achieve optimal stimulation fidelity (Supplementary Figure 9). To our knowledge these results represent the fastest light triggered spiking measured to date, and indicate that vf-Chrimson opens new possibilities for the investigation of high frequency network events such as sharp wave-ripples (Buzsáki, 2015).

\subsection{4 $\mathrm{f}$-Chrimson is a promising candidate for hearing restoration}

Optogenetics bears great potential for improving the restoration of vision and hearing (Moser, 2015a; Sahel and Roska, 2013). Future oCIs shall use tens to hundreds of microscale light sources to focally stimulate tonotopically-ordered SGNs in Rosenthal's canal (Figure 4.4A; (Moser, 2015a)). For deaf people, the lower spread of excitation from the light source in oCIs (Hernandez et al., 2014b), promises improved frequency and intensity resolution when compared to the eCI (Moser, 2015a). However, much remained to be done prior to a potential clinical translation of the oCI. For example, so far cochlear optogenetics was established using blue ChRs expressed in transgenic rodents or in mice following prenatal viral-gene transfer (Hernandez et al., 2014b).

Here, we tested the potential of f-Chrimson for optogenetic stimulation of SGNs. We established postnatal viral gene transfer by injecting AAV2/6-hSyn-f-Chrimson-EYFP into the scala tympani via the round window in 3-6 day-old mice (Figure 4.4B). We readily observed photocurrents in patch-clamp recordings from isolated SGNs (Smith et al., 2015) in the second postnatal week (Figure 4.4C), proving the basic functionality of f-Chrimson in the target cells. We then in depth analyzed expression and function 4-14 weeks after injection. Using confocal imaging of EYFP and parvalbumin immunofluorescence in cochlear cryosections we found a 

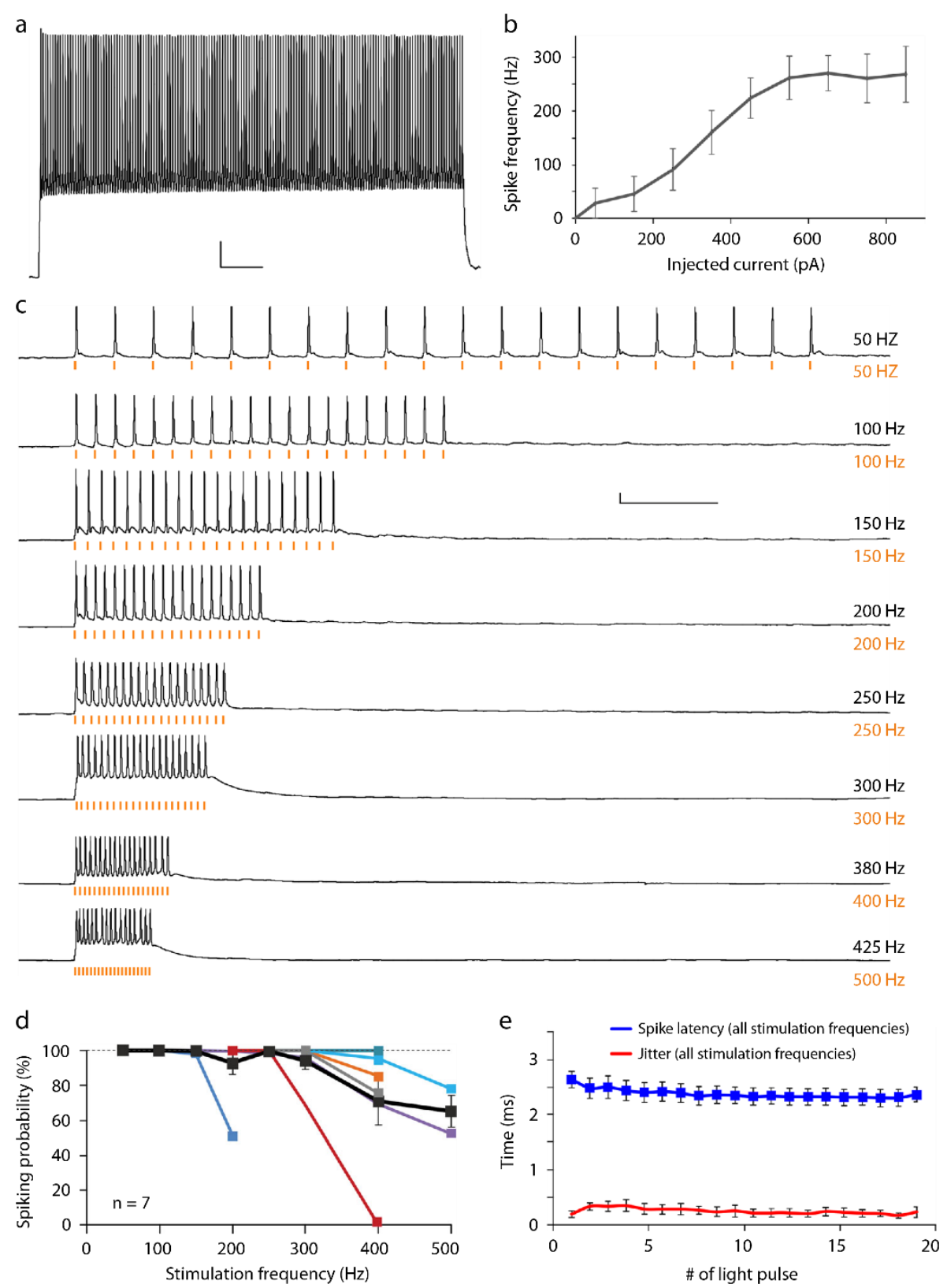

Figure 4.3: vf-Chrimson drives fast interneurons to the frequency limit

A: Example recording of a neocortical parvalbumin-positive interneuron in an acute brain slice. Current injection (500 ms, $550 \mathrm{pA})$ elicits high frequency firing $(322 \mathrm{~Hz})$, consistent with the fast spiking phenotype of these interneurons. B: When tested with constant current injection, the input-output curve of PV-interneurons plateaus at a maximum firing rate of $270 \pm 33 \mathrm{~Hz}$ $(\mathrm{n}=8)$. C: Example traces of the vf Chrimson-expressing PV-interneuron from (A) activated by light pulses $(565 \mathrm{~nm}, 0.5 \mathrm{~ms})$ at frequencies ranging from $50-500 \mathrm{~Hz}$. Note that this interneuron reliably followed frequencies of up to $400 \mathrm{~Hz}$. D: Spiking probabilities of PV-interneurons at different optical stimulation frequencies. On average (black), PV-interneurons followed stimulation up to $300 \mathrm{~Hz}$ reliably ( $94 \pm 5 \%$ spiking probability), and could still encode input frequencies of up to $400 \mathrm{~Hz}$ with a reliability of $68 \pm 16 \%(n=7$; three whole-cell, four cellattached recordings). E: Action potential latency (assessed at peak) and action potential jitter (s.d. of latencies) after light pulse onset for all stimulation frequencies with reliable spiking (>85\%). Error bars are s.e.m. Scale bars: A: $50 \mathrm{~ms}, 10 \mathrm{mV}$; C: $50 \mathrm{~ms}, 10 \mathrm{mV}$. 
high transduction rate (near $80 \%$ ) in the injected ear, which was not significantly different among the cochlear turns (Kruskal-Wallis ANOVA followed by Dunn's test, $p>0.05, \mathrm{n}=5$; Figure 4.4D,F). SGN showed clear plasma membrane expression of f-Chrimson (insets in Figure 4.4F) and survived the optogenetic manipulation as evident from the unaltered SGN density when compared to the non-injected ear (Mann-Whitney $\mathrm{U}$ test, $p>0.05, \mathrm{n}=5$; Figure 4.4E,F). The non-injected ear showed f-Chrimson expression in less than 5\% of the SGNs (Figure 4.4D,F), indicating minimal spread of AAV from the injected ear likely via the cochlear aqueduct.

We then established single-channel oCI stimulation by performing a posterior tympanotomy and inserting an optical fiber $(50 \mu \mathrm{m}$ diameter) through the round window to project the light of a $594 \mathrm{~nm}$ laser onto the SGNs of the basal cochlear turn of young mice (2-3 months, Figure 4.5A). We could readily elicit optical auditory brainstem responses (oABR, Figure 4.5B,C) that differed between animals in waveform and amplitude. For comparison we recorded acoustic auditory brainstem responses (aABRs, Figure 4.5B,C lower panels) that were similar in amplitude and waveform to oABR and also varied between animals (Figure 4.5B). We note that the similarity to aABRs and the shorter latency $(0.93 \pm 0.13 \mathrm{~ms}$ vs. approximately $3 \mathrm{~ms}$ (Hernandez et al., 2014a)) and smaller maximal amplitude (10.7 $\pm 3.80 \mu \mathrm{V}$ vs. approximately $1000 \mu \mathrm{V}$ (Hernandez et al., 2014a)) of oABRs when compared to our previous report on transgenic mice indicates more specific activation of the auditory pathway in the case of postnatal AAV-injection used in present study. We then characterized the oABRs in response to different light intensities, light pulse durations and light pulse rate $(n=5$ mice). oABR amplitude grew and oABR latency got shorter with increasing light intensity (Figure 4.5C,D,G). Stimuli as weak as $0.5 \mathrm{~mW}$ (Figure 4.5C,D; duration: $1 \mathrm{~ms}$, rate: $20 \mathrm{~Hz}$ ) and as short as $80 \mu \mathrm{s}$ (Figure 4.5E,H; rate: $20 \mathrm{~Hz}$, intensity: $11 \mathrm{~mW}$ ) were sufficient to drive oABRs. Amplitudes typically varied for changes in light intensity of more than one order (Figure 4.5C,D, output dynamic range > $10 \mathrm{~dB}(\mathrm{~mW})$ for oABR). oABR amplitudes declined when raising stimulus rates (Figure 4.5F,I). However, f-Chrimson-mediated oABRs remained sizable up to stimulus rates of $200 \mathrm{~Hz}$, suggesting high temporal fidelity of light-driven SGN firing. Next, we used aged C57BL6/J mice (9 months-old, $\mathrm{n}=5$ mice) to explore the potential of oCI to restore activity in the auditory pathway of a mouse model of age-related hearing loss (Shnerson and Pujol, 1981), which is a major form of hearing impairment in humans. Auditory thresholds, estimated 

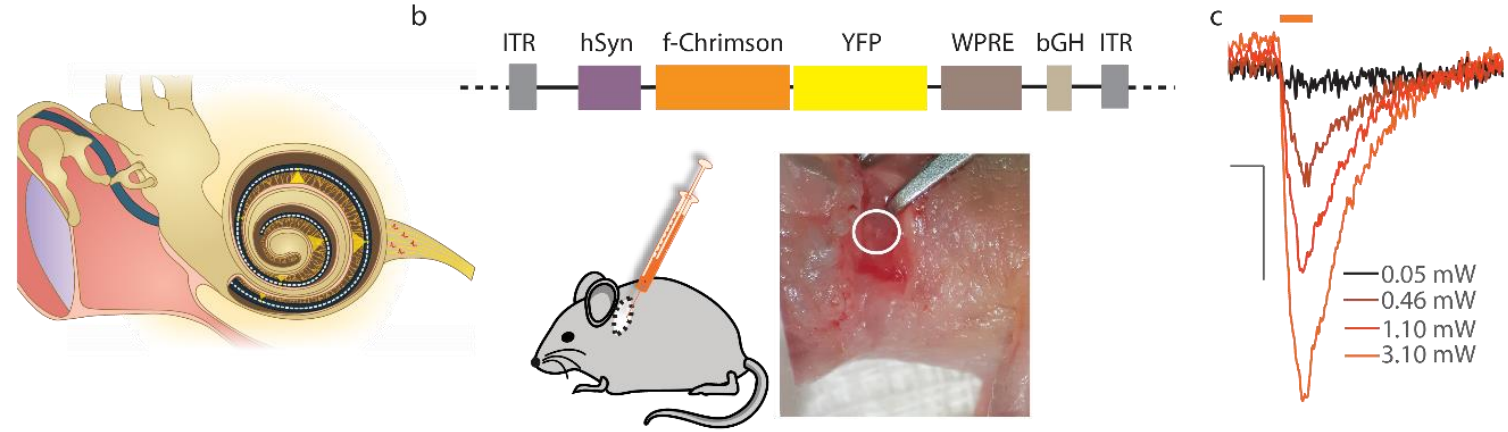

d
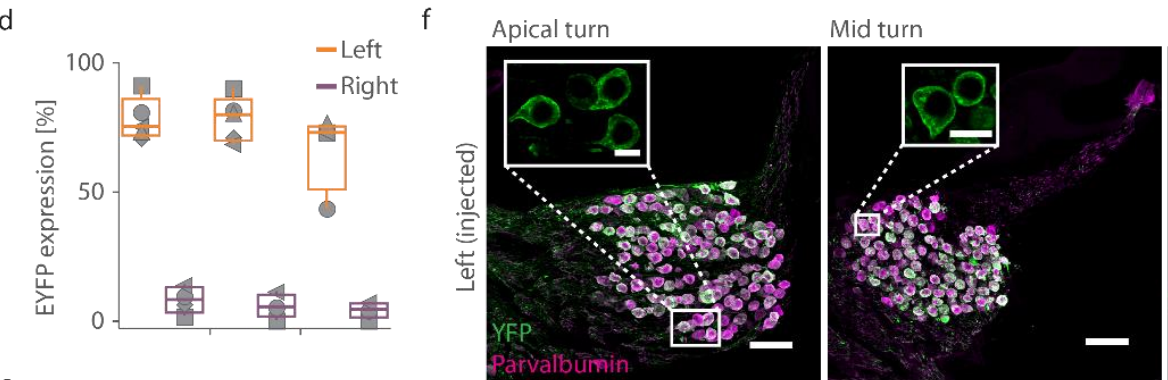

Basal turn

e
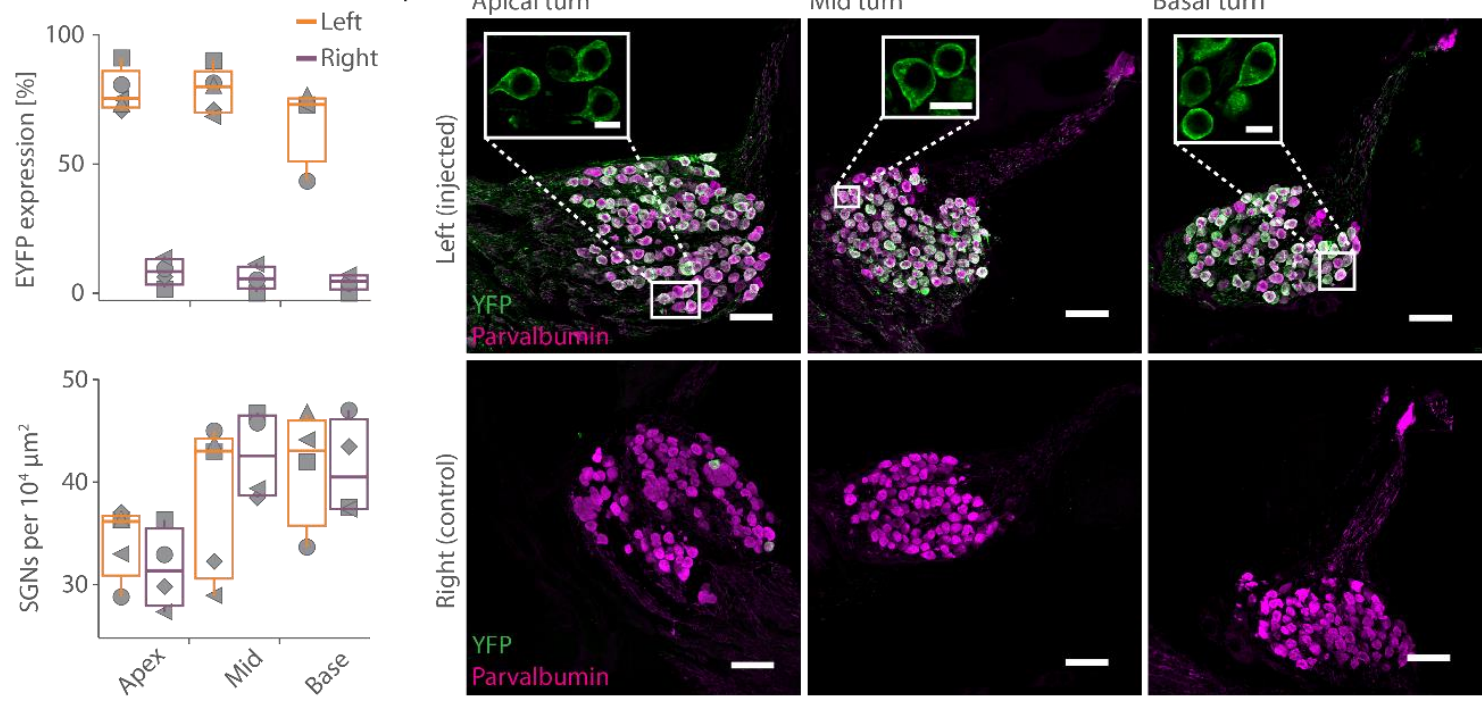

Figure 4.4: f-Chrimson expression after postnatal AAV-transduction of SGNs

A: Scheme of the future oCI as implanted into the human ear: the oCI passes through the middle ear (limited left by ear drum and right by inner ear) near the ossicles, enters the cochlea and spirals up in scala tympani. It will likely contain tens of microscale emitters (orange spots on oCI) that stimulate (orange beams) SGNs housed in the modiolus (central compartment of the cochlea), that encode information as APs. SGNs form the auditory nerve (right) which carries the information to the brain (not displayed). B: pAAV vector used in the study to express fChrimson-EYFP under the control of the hSynapsin promoter (top) upon early postnatal injection of AAV2/6 into scala tympani via a posterior tympanotomy (lower left) to expose the round window (white circle in right lower panel). C: Photocurrents of a representative culture f-Chrimson-EYFP-positive SGN isolated from an injected ear at P14. Light pulses of $2 \mathrm{~ms}$ duration were applied at the indicated intensities in the focal plane and photocurrents recorded at $-73 \mathrm{mV}$ at room temperature. Scale bar: $2 \mathrm{~ms}, 50 \mathrm{pA}$. D: Fraction of EYFP-positive SGNs (identified by parvalbumin immunofluorescence, parvalbumin ${ }^{+}$) and $\mathbf{E}$ : density of parvalbumin ${ }^{+}$ SGNs (\#cells per $10^{4} \mu \mathrm{m}^{2}$ ) obtained from data as in F. Symbols mark results from individual animals $(n=5)$, box-whisker plots show 10th, 25th, 50th, 75th and 90th percentiles of the injected (orange) and non-injected control (magenta) cochleae (Kruskal-Wallis ANOVA, $p=0.6538, H=0.98$; post-hoc Dunn's test for comparison of expression, $p>0.05$ for all pairwise comparisons; Mann-Whitney U test for comparison of density, $L_{\text {apex }}$ Vs. $R_{\text {apex }}, L_{\text {mid }}$ Vs. $R_{\text {mid }}, L_{\text {base }}$ Vs. $R_{\text {base, }} p>0.05$ for all comparisons). F: Projections of confocal cryosections with YFP (green) and parvalbumin (magenta) immunofluorescence of SGNs in three cochlear regions (scale bar: $50 \mu \mathrm{m}$ ). Insets (scale bar: $10 \mu \mathrm{m}$ ) show close-up images of single z-sections of the same images. 
by aABR elicited by acoustic clicks were elevated to above $50 \mathrm{~dB}$ (SPL) $(58 \pm 3.3 \mathrm{~dB}$ SPL, Figure 4.5J-L, typically $20 \mathrm{~dB}$ in young mice) and aABR amplitudes were reduced to $1 / 3$ of those in young mice across all SPLs tested (Figure 4.5L). oABR amplitudes measured in these aged mice were comparable to values obtained for young animals (Figure 4.5L), but latencies tended to be shorter and less variable (Figure 4.5G and Supplementary Figure 10H). Interestingly, we found that light pulses as short as $40 \mu \mathrm{s}$ were able to elicit oABRs $(11 \mathrm{~mW}$ at $20 \mathrm{~Hz}$, Supplementary Figure 10F,I) as compared to $80 \mu \mathrm{s}$ in young mice (Figure 4.5E). Moreover, we were able to record oABRs at stimulation frequencies as high as $250 \mathrm{~Hz}(11 \mathrm{~mW}$, $1 \mathrm{~ms}$ pulse duration), likely due to the lower average latencies found in these aged mice as compared to their younger counterparts (Supplementary Figure 10G,J). Together the data indicate that optical activation of the auditory pathway proceeded with at least as high efficiency in aged C57BL/6J mice despite their profound age-related hearing impairment. f-Chrimson expression levels throughout the injected cochlea were homogeneous (one-way ANOVA followed by Tukey's test, $p>0.05, \mathrm{n}=5$ ). Importantly, long-term f-Chrimson expression (9 months) did not seem to decay significantly (Kruskal-Wallis ANOVA followed by Dunn's test, $p>0.05, \mathrm{n}=5$ ) (Supplementary Figure 10A-C) nor cause any significant loss of SGNs in the AAV-injected ear of these mice, when compared to the non-injected ear (t-test for comparison of cell density across cochlear turns in the injected and non-injected ear, $p>0.05, \mathrm{n}=5$ ) (Supplementary Figure 10D).

In order to scrutinize the temporal fidelity of stimulation, we turned to juxtacellular recordings from single neurons (Liberman, 1978; Miller et al., 2008). We established single-channel oCI stimulation via an optical fiber and targeted electrodes through a craniotomy to where the auditory nerve enters the cochlear nucleus (Figure 4.6A) in order to measure the neural photoactivation. Those neurons could not be identified based on a response to acoustic stimulation, most likely due to impaired acoustic hearing following ear surgery and oCI. Therefore, we termed light-stimulated neurons putative SGNs. We found that the putative SGNs fired upon optogenetic stimulation with high temporal precision for stimulus rates of up to hundreds of $\mathrm{Hz}$ (Figure 4.6B-E): some neurons followed stimulation to some extent even up to $1 \mathrm{kHz}$ (Figure 4.6B,D). The spike latency amounted to approximately $2 \mathrm{~ms}$ for stimulus rates of up to $400 \mathrm{~Hz}$ (Supplementary Figure 11A,B), which is in agreement with the data obtained on 

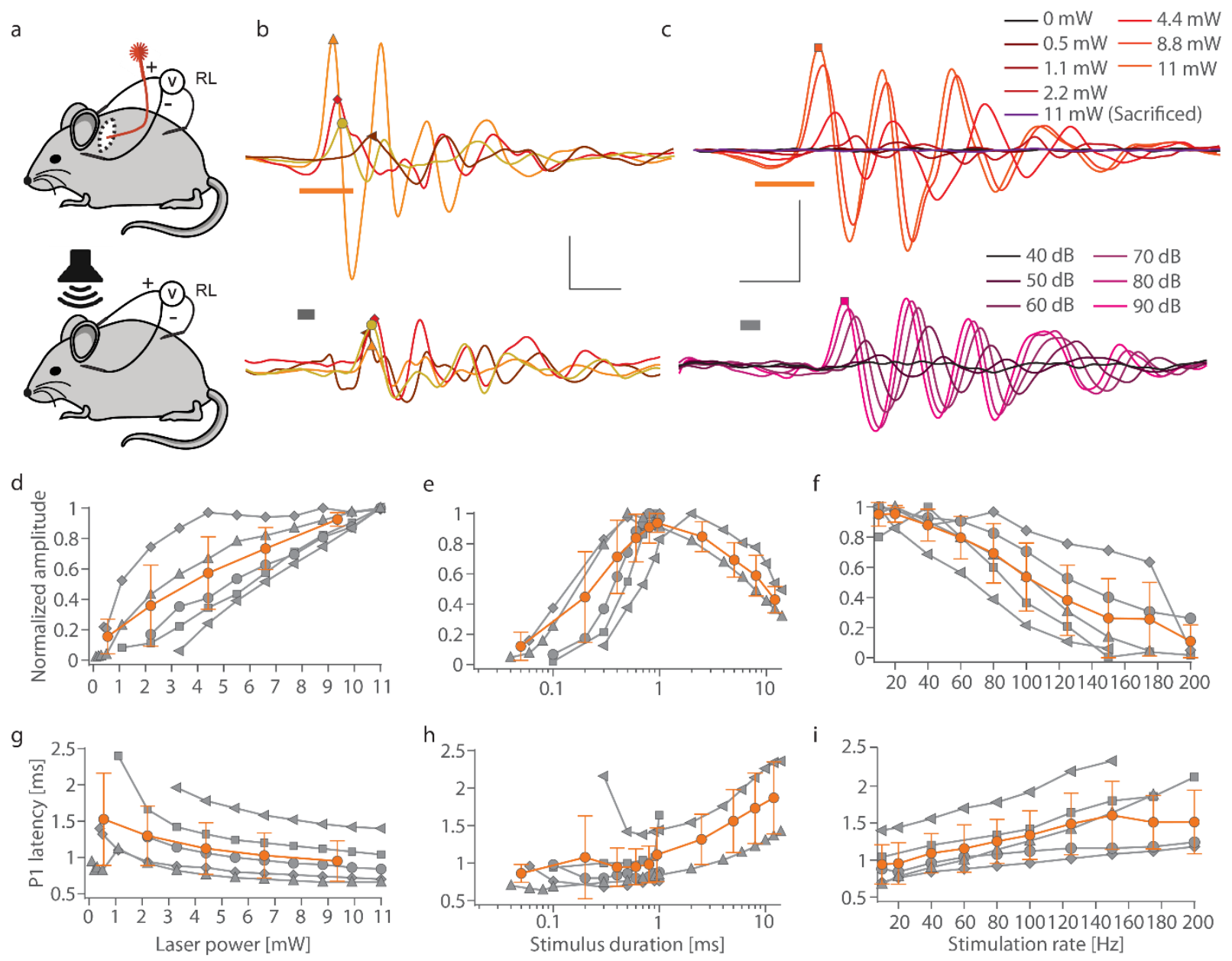
j

$\mathrm{k}$
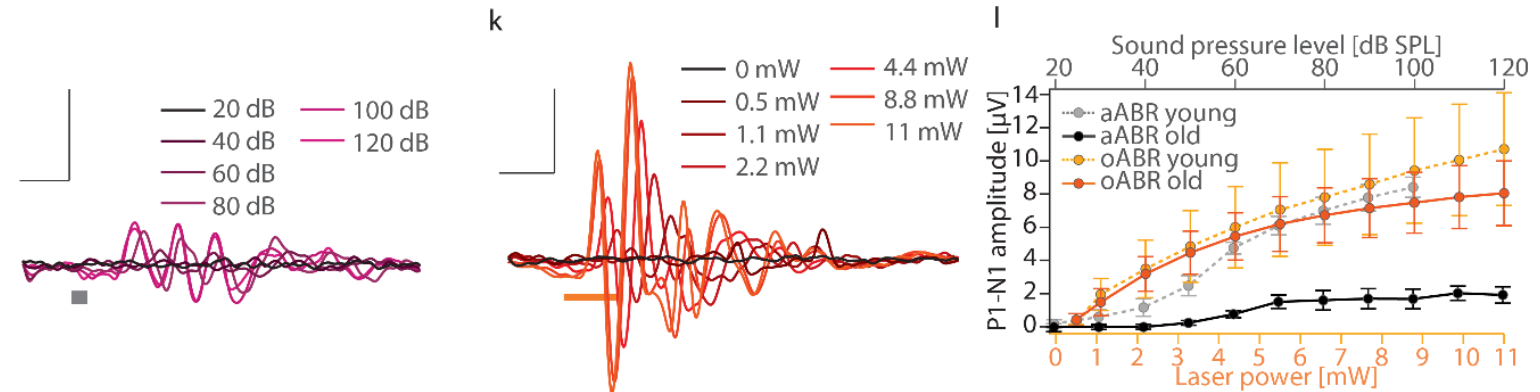

Figure 4.5: Single-channel oCIs drive oABRs in hearing and deaf mice

A: Experimental set-up for oABR-recordings in mice: a $50 \mu \mathrm{m}$ optical fiber coupled to a $594 \mathrm{~nm}$ laser was implanted into scala tympani via a posterior tympanotomy and the round window. Recordings of far-field optically evoked potentials were performed by intradermal needle electrodes. For aABR recordings a free-field speaker was employed (lower panel). B: Comparing oABRs (upper panel) and aABRs (lower panel) at strong stimulation levels for four mice (average of 1000 trials). oABRs were recorded in response to $1 \mathrm{~ms}$ long, $11 \mathrm{~mW}, 594 \mathrm{~nm}$ laser pulse at $10 \mathrm{~Hz}$, aABRs of the same mice in response to $80 \mathrm{~dB}$ (SPL peak equivalent) clicks. Bars indicate the stimulus timing. C: oABRs (upper panel, $594 \mathrm{~nm}, 1 \mathrm{~ms}$ at $10 \mathrm{~s}^{-1}$ ) and aABRs (lower panel, clicks at $10 \mathrm{~s}^{-1}$, values in SPL [peak equivalent]) recorded from an exemplary AAV-injected mouse at increasing stimulus intensities. D-F: Normalized P1-N1-amplitude as a function of laser intensity (D: $1 \mathrm{~ms}$ at $20 \mathrm{~Hz}$ ), pulse duration (E: $11 \mathrm{~mW}$ at $20 \mathrm{~Hz}$ ), and stimulus rate $(\mathbf{F}: 11 \mathrm{~mW}, 1 \mathrm{~ms})$. Group average (lines) and s.d. (error bars) are shown in orange (same for G-I). G-I: P1-latency as a function of laser intensity ( $\mathbf{G}$ as in D), duration ( $\mathbf{H}$ as in E), and 
$\leftarrow \quad$ rate (I as in F). J: Exemplary aABR recordings done as in A-C using a 9 months-old mouse (following postnatal AAV-Chrimson-EYFP injection: elevated acoustic thresholds (around $60 \mathrm{~dB}$ [SPL], compare to $\mathrm{C}$ ). $\mathrm{K}$ : oABR recordings done as in A-C in the same mouse as in $\mathrm{J}$, using $1 \mathrm{~ms}$ long laser pulses: thresholds similar to injected mice at 2-3 months of age (around $1 \mathrm{~mW}$, compare to C). L: P1-N1-amplitude of oABR (orange) and P1-N1-amplitude of aABR (gray) as function of stimulus intensity in young (2-3 months-old) and old (9 months-old) mice $(\mathrm{n}=5$ for each group, means (lines) \pm s.e.m. (error bars) are shown. Symbols in D-I mark results from individual animals. Scale bars $(\mathrm{B}, \mathrm{C}, \mathrm{J}, \mathrm{K}): 1 \mathrm{~ms}, 5 \mu \mathrm{V}$.

the interneurons (Figure 4.3E). Temporal precision of firing, evaluated based on vector strength ((Goldberg and Brown, 1969), see methods, Figure 4.6C,D) and temporal jitter (i.e., standard deviation of spike latency across trials, Figure 4.6E, Supplementary Figure 11C) varied between the recorded neurons and, generally, was good. The vector strength declined with increasing stimulation rate up to $1 \mathrm{kHz}$. For a comparison, we re-plot the median vector strength of firing driven by transposed tones in mouse SGNs ((Buran et al., 2010); Figure 4.6D) used because phase-locking to pure tones is hard to achieve in the high frequency mouse cochlea (Taberner and Liberman, 2005). Temporal jitter, evaluated for spikes occurring in the time window equal to the stimulus period, was typically below a millisecond and tended to increase when raising stimulus rates up to $300 \mathrm{~Hz}$ (Figure 4.6E). At higher stimulus rates, the temporal jitter was higher than the values obtained for simulated Poisson spike trains (see methods, gray area, Figure 4.6E), reflecting a reduced spike synchronization with the light pulses. Interestingly, the spike jitter increased significantly from $25 \mathrm{~ms}$ compared to the start of the stimulation (Supplementary Figure 11C). Spike probability (Figure 4.6C-D) declined as the rate of stimulation increased, indicating that optogenetic coding by individual SGNs becomes less reliable as stimulus rate rises. This, however, is likely compensated at the population level, as several SGNs jointly encode information from each place of the tonotopic map (Liberman, 1978).

\subsection{Discussion}

As demonstrated, the investigation of the molecular properties of microbial-type rhodopsins is essential for the development of variants with superior properties for particular optogenetic applications. Our study reveals the critical role of helix $\mathrm{F}$ for the closing kinetics of various ChRs. Using site-directed mutagenesis we generated fast variants of four different ChRs, which, together, cover photoactivation over the visible spectrum. We deem the fast (f-) and very fast 


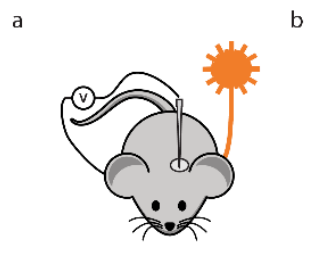

b
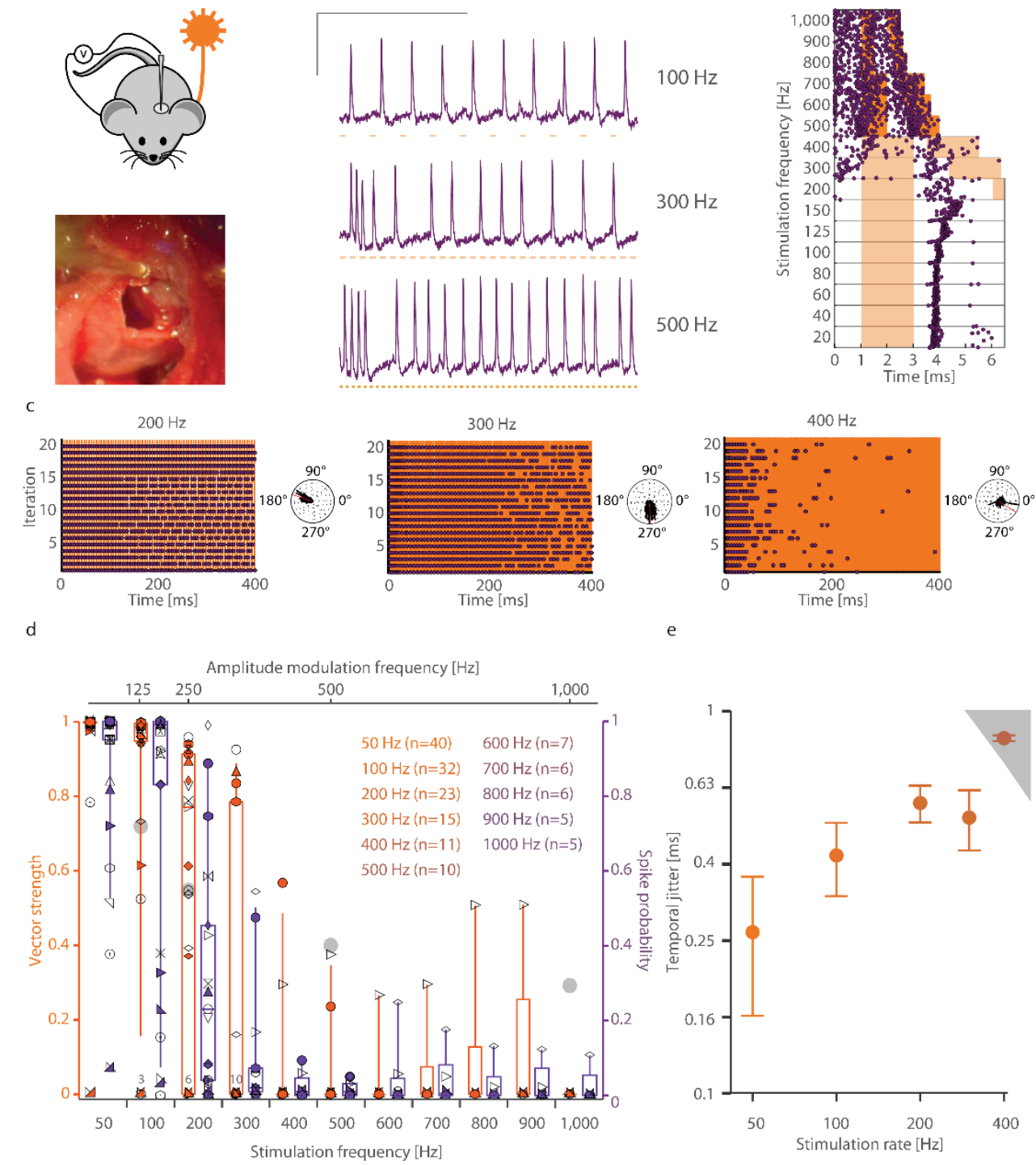

e

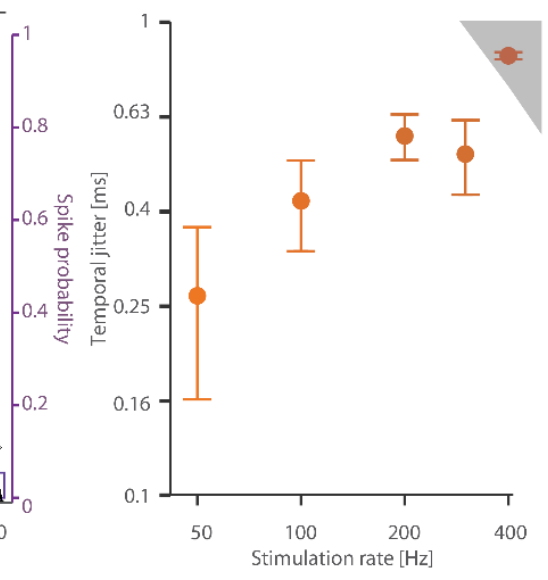

Figure 4.6: f-Chrimson enables SGNs spiking at near physiological rates

A: Experimental set-up for recording optogenetic responses of SGNs in mice: a $50 \mu \mathrm{m}$ optical fiber coupled to a $594 \mathrm{~nm}$ laser was implanted into scala tympani via the round window (lower panel, see cylindrical structure in the upper half) and microelectrodes were advanced into the cochlear nucleus via a craniotomy (upper panel). B: Exemplary spikes of a neuron (1 ms, $5.5 \mathrm{~mW}$ for $100,300 \mathrm{~Hz} ; 11 \mathrm{~mW}$ for $500 \mathrm{~Hz}$ ). Raster plot (right panel): spike times in response to laser pulses (orange bars: $2 \mathrm{~ms} @ 5.5 \mathrm{~mW}$ for 20-400 Hz, $1 \mathrm{~ms} @ 11 \mathrm{~mW}$ for 500-700 Hz and above:0.5 ms@11 mW): spikes cluster in time for stimulus rates up to hundreds of $\mathrm{Hz}$, temporal jitter increases with stimulation rates. Scale bar: $50 \mathrm{~ms}, 2 \mathrm{mV}$. C: Activity of an exemplary neuron in response to $900 \mathrm{~ms}$ trains of laser pulses $(1 \mathrm{~ms})$ at three different rates leaving an inter-train recovery time of $100 \mathrm{~ms}$ (first $400 \mathrm{~ms}$ are shown and analyzed). Panels to the right side of raster plots show polar plots: synchronicity and probability of firing decay with increasing stimulus frequency. Spike probability $200 \mathrm{~Hz}: 0.8,300 \mathrm{~Hz}: 0.33,400 \mathrm{~Hz}: 0.04$. Vector strength $200 \mathrm{~Hz}$ : 0.92, $300 \mathrm{~Hz}: 0.83$, $400 \mathrm{~Hz}: 0.57$ (Rayleigh-test: $p<0.001$ in all cases). D: Box-whisker plots showing 10th, 25th, 50th, 75th and 90th percentiles of the vector strength (orange) and spike probability (purple) of 40 units from five mice, stimulated at different rates as described for C. Symbols represent values from every unit. Gray circles are means of vector 
$\leftarrow$ strength of SGNs in wild-type mice found with transposed tones at the characteristic frequency at $30 \mathrm{~dB}$ relative to spike threshold (Taberner and Liberman, 2005), for comparison. Numbers at the bottom of the graph indicate number of units clustered below them. E: Temporal jitter of spikes across stimulation rates $50-400 \mathrm{~Hz}$. Gray area represents the hazard function obtained in response to simulated Poisson spike trains. Data points show mean (lines) \pm s.e.m. (error bars). Number of units included for each stimulation frequency (color coded) is shown.

(vf-) Chrimson of particular interest to the neurosciences because of their red-shifted action spectrum and high membrane expression. Our analysis of fast spiking interneurons of the cerebral cortex demonstrated that they enable the remote optical control of even the fastest neurons at their intrinsic physiological limits. Finally, we show that f-Chrimson is a promising candidate for future clinical optogenetic restoration of sensory function.

Channel opening/closing of ChR2 is based on a concerted movement of helices $\mathrm{B}, \mathrm{F}$ and $\mathrm{G}$ (Müller et al., 2015; Sattig et al., 2013). Interestingly, we discovered a major impact of the interaction between the moving helix $\mathrm{F}$ and the virtually immobile helix $\mathrm{C}$ on ChR kinetics. High resolution structures of the investigated ChRs are not available. However, the information of the high resolution structure of the $\mathrm{C} 1 \mathrm{C} 2 \mathrm{ChR}$ chimera in combination with the light-induced helix movement studies by electron spin resonance and the low-resolution structure by 2D cryoelectron microscopy allowed us to identify the crucial position F219 in helix $F$ for the construction of a faster ChR2 mutant. This position is also conserved in ReaChR and VChR1. Analogous mutations lead to accelerated kinetics of channel closing. Of note, the surprisingly slow reacting Chrimson has already a tyrosine on this position (Figure 4.1A,B). We suspected that the mutation of this tyrosine influences channel closing kinetics. Indeed, the mutation to a phenylalanine in the analogous position (Y261F) results to faster channel closing in Chrimson. Further inspection of the alignment of helix F shows at positions M225 and F226 in ChR2 the same analogy for VChR1 and ReaChR but not for Chrimson. For Chrimson, mutations on these positions (S267M, Y268F) in addition to the Y261F mutation result in the ultrafast switching behavior (Fig 4.1G,H). The surprising and peculiar phenotype of the back mutations in Chrimson is hard to explain without a high resolution structure of the protein.

This study achieved two important breakthroughs towards developing cochlear optogenetics for auditory research and future improved hearing restoration. First, we managed to achieve efficient, non-traumatic and neuron-specific expression of f-Chrimson in SGNs using postnatal AAV-injection into scala tympani through the round window. We found near $80 \%$ of the SGNs 
in the injected ear to express f-Chrimson at high levels and mostly in the plasma membrane of somas and neurites, which persists for at least 9 months after injection. These transduction rates were much higher than those achieved with transuterine injection of AAV2/6-hSyn-CatCh-YFP (Hernandez et al., 2014b) and unlike there, independent from tonotopic position. We consider the minute transduction of the non-injected ear to reflect viral spread through the cerebrospinal fluid space, which calls for further optimization of the injection protocol (Lalwani et al., 1996). Importantly, we did not find evidence for neuron loss even at 9 months after injection and we expect little, if any, risk of phototoxicity given the red-shifted action spectrum of f-Chrimson.

Secondly, using f-Chrimson, we overcame the likely biggest roadblock of current ChR2-based cochlear optogenetics: low temporal bandwidth of optical coding $(<80 \mathrm{~Hz})$ (Hernandez et al., 2014b). We found robust and fast photocurrents in cultured isolated f-Chrimson-positive SGNs. In vivo, fiber-based stimulation resembling single-channel oCI elicited activation of the auditory pathway in hearing and deaf mice. Using far-field neural population responses (oABR) as a readout we found low thresholds for radiant flux and energy $(<0.5 \mathrm{~mW},<0.5 \mu \mathrm{J})$ as well as duration $(<100 \mu \mathrm{s})$ and a wide dynamic range of coding (more than $10 \mathrm{~dB}(\mathrm{~mW})$, no saturation for most animals at maximal stimulation). This brings the oCI closer to the currently used eCI stimulation parameters $(0.2 \mu \mathrm{J}$ and $80 \mu$ s per pulse) (Zierhofer et al., 1995) and exceeds the eCI output dynamic range (<10 dB) (Zeng et al., 2008). Both, recordings of oABR and of firing in single putative SGNs indicated that f-Chrimson mediated oCI-enabled responses to follow at least $200 \mathrm{~Hz}$, which corresponds to physiological steady-state firing rates of SGNs (Liberman, 1978). In fact, we found that some neurons follow stimulation to several hundreds of $\mathrm{Hz}$, nearly mimicking sound-evoked SGN activity. The closing kinetics of f-Chrimson and the resulting relative refractoriness probably also limits the temporal precision of f-Chrimson-mediated SGN firing. At 500 and $1000 \mathrm{~Hz}$, vector strength, a measure commonly used to analyze the extent of phase-locking in SGNs (Köppl, 1997), was lower for f-Chrimson-mediated optogenetic stimulation than for mouse SGN firing with transposed tones (Buran et al., 2010). We note that the SGNs recorded in the present study typically did not fire spontaneously probably due to the ear surgery. Besides the lack of spontaneous firing, the short (1 ms) and pulsatile optogenetic stimulation typically evoking a single spike likely explain why vector strength tended to be higher for low stimulus rates when compared to transposed tones, for which several spikes were generated per stimulus cycle. Moreover, vector strength and temporal jitter of f-Chrimsonmediated SGN firing in mice indicate a lower temporal precision than that of acoustic hearing 
and electric stimulation in species with prominent phase-locking of SGN firing (Köppl, 1997; Miller et al., 2008). Nonetheless, we reason that, even if the limited probability and temporal precision of single SGN firing for optogenetic stimulation at 100-500 Hz translates to species other than the mouse, this will not impede the coding at the level of the auditory nerve population. Hence, we conclude that f-Chrimson is a good candidate opsin for the oCI. In fact, higher temporal jitter in response to optogenetic than electrical stimulation might render unnecessary the very high stimulation rates employed in eCI to avoid overly synchronized activity in the auditory nerve (Zeng et al., 2008). The favorable properties of the novel Chrimson mutants also facilitate multiple applications in basic neurosciences and in sensory restoration, such as the recovery of vision (Sahel and Roska, 2013).

\subsection{Methods}

\section{Molecular biology}

The humanized DNA sequence coding for the red light activated ChR Chrimson from Chlamydomonas noctigama (accession number: KF992060), either C-terminally fused to EYFP or without a fluorescent tag, was cloned into the mammalian expression vector pcDNA3.1(-) (Invitrogen, Carlsbad, USA). The mutations L174C, K176R (ChrimsonR), Y261F, S267M and Y268F as well as combinations of aforementioned mutations (Table 4.1) were created by sitedirected mutagenesis. Chrimson-EYFP wt and Chrimson-EYFP Y261F/S267M (f-ChrimsonEYFP) were subcloned into the Xenopus laevis oocyte expression vector pTLN (Lorenz et al., 1996).

We also cloned the humanized DNA sequences coding for ChR2 (C-terminally truncated variant Chop2-315 of ChR2 from Chlamydomonas reinhardtii, accession number: AF461397), for Volvox ChR1 (VChR1, accession number: EU622855) and for the chimera ReaChR (ChR1/VChR1/VChR2, Red-activatable ChR, accession number: KF448069) into the mammalian expression vector pcDNA3.1 (-) (Invitrogen, Carlsbad, USA). Thereby ChR2 and VChR1 were C-terminally fused to EYFP and ReaChR was C-terminally fused to citrine. The mutants ChR2-EYFP F219Y, VChR1-EYFP F214Y, VChR1-EYFP L127C, ReaChR-Citrine F259Y and ReaChR-Citrine L172C were created by site-directed mutagenesis. The related primer sequences are shown in Supplementary Tables 5 and 6. 


\section{NG108-15 cell culture and transfection}

NG108-15 cells (ATCC, HB-12377 ${ }^{\mathrm{TM}}$, Manassas, USA) were cultured at $37^{\circ} \mathrm{C}$ and $5 \% \mathrm{CO}_{2}$ in DMEM (Sigma, St. Louis, USA) supplemented with 10\% fetal calf serum (Sigma, St. Louis, USA), and $5 \%$ penicillin/streptomycin (Sigma, St. Louis, USA). One day prior to transient transfections the NG108-15 cells were seeded on 24-well plates. Two to three days prior to their electrophysiological characterization by patch-clamp experiments the NG108-15 cells were transiently transfected with pcDNA3.1(-) derivatives carrying aforementioned ChRs and $\mathrm{ChR}$ mutants using Lipofectamine 2000 (Invitrogen, Carlsbad, USA) or Lipofectamine LTX (Invitrogen, Carlsbad, USA). Cells were tested for mycoplasma contamination using specific primers. No method of cell line authentication was used.

\section{Expression of Chrimson variants in Xenopus laevis oocytes}

Xenopus laevis oocytes were injected with $50 \mathrm{ng}$ of in vitro-transcribed cRNA (Thermo Fisher Scientific, Waltham, USA), coding for Chrimson-EYFP wt and Chrimson-EYFP Y261F/S267M (f-Chrimson-EYFP). After cRNA injection the Xenopus laevis oocytes were incubated at $16^{\circ} \mathrm{C}$ in an $1 \mu \mathrm{M}$ all-trans retinal containing Barth's solution $(88 \mathrm{mM} \mathrm{NaCl}, 1 \mathrm{mM}$ $\mathrm{KCl}, 0.33 \mathrm{mM} \mathrm{Ca}\left(\mathrm{NO}_{3}\right)_{2}, 0.41 \mathrm{CaCl}_{2}, 0.82 \mathrm{MgSO}_{4}, 2.4 \mathrm{mM} \mathrm{NaHCO} 3,10 \mathrm{mM}$ HEPES, pH 7.4 supplemented with $50 \mathrm{mg} / \mathrm{l}$ gentamycin, $67 \mathrm{mg} / \mathrm{l}$ penicillin and $100 \mathrm{mg} / \mathrm{l}$ streptomycin) for 4-5 days.

\section{Electrophysiological recordings on Xenopus laevis oocytes}

The Xenopus laevis oocytes heterologously expressing the Chrimson mutants were investigated by the two electrode voltage-clamp techniques (Nagel et al., 1995, 2003). Photocurrents were measured in response to $500 \mathrm{~ms}$ light pulses with a wavelength of $\lambda=590 \mathrm{~nm}$ using the LED OEM module (Omikron, Rodgau-Dudenhofen, Germany) focused into a $2 \mathrm{~mm}$ optical fiber. In order to assess the permeability of potassium ions relative to the permeability of sodium ions $\left(\mathrm{P}_{\mathrm{K}} / \mathrm{P}_{\mathrm{Na}}\right)$, we measured photocurrents at voltages ranging from $-120 \mathrm{mV}$ to $+40 \mathrm{mV}$ in $20 \mathrm{mV}$ steps. The $\mathrm{P}_{\mathrm{K}} / \mathrm{P}_{\mathrm{Na}}$ ratio was determined from the difference of the reversal potentials of the photocurrents when replacing $90 \mathrm{mM} \mathrm{NaCl}, 2 \mathrm{mM} \mathrm{MgCl}_{2}, 5 \mathrm{mM}$ MOPS/TRIS pH 9 for $90 \mathrm{mM}$ $\mathrm{KCl}, 2 \mathrm{mM} \mathrm{MgCl}$, $5 \mathrm{mM}$ MOPS/TRIS pH 9 (Nagel et al., 2003). The relative proton permeability was calculated from the photocurrent reversal potential in buffer containing 90 
mM NMG, $5 \mathrm{mM} \mathrm{KCl,} 2 \mathrm{mM} \mathrm{MgCl} 2,5 \mathrm{mM}$ MOPS/TRIS pH 9 using the Goldmann-HodgkinKatz equation (Hille, 2001) and assuming a cytoplasmic potassium concentration of $100 \mathrm{mM}$ and an intracellular pH of 7.3 (Nagel et al., 2003).

\section{Electrophysiological recordings on NG108-15 cells}

For the electrophysiological characterization of mutant channelrhodopsins whole cell patchclamp were performed under voltage clamp conditions (Sakmann and Neher, 1995) using the Axopatch 200B amplifier (Axon Instruments, Union City, USA) and the DigiData 1322A interface (Axon Instruments, Union City, USA). Patch pipettes with resistances of 2-5 M $\Omega$ were fabricated from thin-walled borosilicate glass on a horizontal puller (Model P-1000, Sutter Instruments, Novato, USA). The series resistance was $<10 \mathrm{M} \Omega$ and the input resistance ranged from $1.1 \mathrm{G} \Omega$ to $4.6 \mathrm{G} \Omega$. The mean capacitance of the measured cells was $34.6 \pm 24.3 \mathrm{pF}(\mathrm{n}=$ 61). If not stated differently the pipette solution contained $110 \mathrm{mM} \mathrm{NaCl}, 2 \mathrm{mM} \mathrm{MgCl} 2,10 \mathrm{mM}$ EGTA, $10 \mathrm{mM}$ HEPES, pH 7.4 and the bath solution contained $140 \mathrm{mM} \mathrm{NaCl}, 2 \mathrm{mM} \mathrm{CaCl} 2,2$ $\mathrm{mM} \mathrm{MgCl}_{2}, 10 \mathrm{mM}$ HEPES, pH 7.4.

In order to assess the permeability of calcium ions relative to the permeability of sodium ions $\left(\mathrm{P}_{\mathrm{Ca}} / \mathrm{P}_{\mathrm{Na}}\right)$, we measured photocurrent-voltage relationships and determined the reversal potential. The intracellular solution contained $110 \mathrm{mM} \mathrm{NaCl}, 10 \mathrm{mM}$ EGTA, $2 \mathrm{mM} \mathrm{MgCl} 2$ and $10 \mathrm{mM}$ Tris $(\mathrm{pH}=7.4)$ and the extracellular solution contained $140 \mathrm{mM} \mathrm{NaCl}, 2 \mathrm{mM} \mathrm{MgCl} 2$ and $10 \mathrm{mM}$ Tris $(\mathrm{pH}=9)$. For the determination of the $\mathrm{P}_{\mathrm{Ca}} / \mathrm{P}_{\mathrm{Na}}$ values, external $140 \mathrm{mM} \mathrm{NaCl}$ was exchanged with $90 \mathrm{mM} \mathrm{CaCl}$. Permeability ratios were calculated according to the Goldman-Hodgkin-Katz equation (Hille, 2001).

For determination and comparison of the off-kinetics and current densities, NG108-15 cells heterologously expressing aforementioned $\mathrm{ChRs}$ and $\mathrm{ChR}$ mutants were investigated at a membrane potential of $-60 \mathrm{mV}$. Photocurrents were measured in response to $3 \mathrm{~ms}$ or $500 \mathrm{~ms}$ light pulses with a saturating intensity of $23 \mathrm{~mW} / \mathrm{mm}^{2}$ using diode-pumped solid-state lasers $(\lambda$ $=473 \mathrm{~nm}$ for ChR2 variants, $\lambda=532 \mathrm{~nm}$ for VChR1 and ReaChR variants, $\lambda=594 \mathrm{~nm}$ for Chrimson variants) focused into a $400-\mu \mathrm{m}$ optical fiber. Light pulses were applied by a fast computer-controlled shutter (Uniblitz LS6ZM2, Vincent Associates, Rochester, USA).

The current density $\left(\mathrm{J}_{-60 \mathrm{mV}}\right)$ was determined by dividing the stationary current in response to a $500 \mathrm{~ms}$ light pulse with a saturating intensity of $23 \mathrm{~mW} / \mathrm{mm}^{2}$ by the capacitance of the cell. In 
order to avoid an experimental bias, the NG108-15 cells for the electrophysiological recordings were chosen independent of the brightness of their EYFP fluorescence. The $\tau_{\text {off }}$ value was determined by a fit of the decaying photocurrent to a monoexponential function. In order to investigate the dependence of the off-kinetics on the membrane potential, $\tau_{\text {off }}$ values were determined at membrane potentials ranging from $-120 \mathrm{mV}$ to $+60 \mathrm{mV}$.

If not stated differently the off-kinetics was determined at room temperature $(297 \mathrm{~K})$. The temperature dependence of the off-kinetics of Chrimson-EYFP wt and Chrimson-EYFP K176R/Y261F/S267M (vf-Chrimson-EYFP) were investigated at temperatures ranging from $284 \mathrm{~K}$ to $307 \mathrm{~K}$. Photocurrents recorded at a temperature of $307 \mathrm{~K}$ were measured in response to $7 \mathrm{~ns}$ light-pulses with a wavelength of $594 \mathrm{~nm}$ in order to avoid tampering of the off-kinetics due to the opening/closing time of the shutter $(700 \mu \mathrm{s})$. The ns light pulses were generated with the Opolette 355 tunable laser system (Opotek Inc, Carlsbad, USA). Thereby the pulse energy was set to $10^{19}$ photons $/ \mathrm{m}^{2}$.

The Opolette 355 tunable laser system was further used for the measurement of the action spectra of the Chrimson variants. For the recordings, the pulse energies at the different wavelengths were set to values which corresponded to equal photon counts of $10^{18}$ photons $/ \mathrm{m}^{2}$ for Chrimson wt and $10^{19}$ photons $/ \mathrm{m}^{2}$ for the Chrimson mutants.

\section{Hippocampal neuron culture}

Hippocampi were isolated from Sprague-Dawley rats at P1 and treated with papain $\left(20 \mathrm{U} \mathrm{ml}^{-1}\right)$ for $20 \mathrm{~min}$ at $37^{\circ} \mathrm{C}$ (by the lab of Dr. Erin Schuman, MPI of Brain Research, Frankfurt). The hippocampi were washed with DMEM high glucose (Sigma-Aldrich, St. Louis, USA) supplemented with $10 \%$ fetal bovine serum and titrated in a small volume of this solution. Approximately 96000 cells were plated on poly-D-lysine/laminin coated glass coverslips in 24well plates. After $3 \mathrm{~h}$, the plating medium was replaced by culture medium containing Neurobasal A (Thermo Fisher Scientific, Waltham, USA) supplemented with 2\% B-27 supplement (Thermo Fisher Scientific, Waltham, USA), and 2 mM Glutamax (Thermo Fisher Scientific, Waltham, USA). 


\section{Adeno-associated virus (AAV2/1) transduction}

rAAV2/1 virus was prepared by the lab of Botond Roska (FMI, Basel, Switzerland) using a pAAV2 vector with a human synapsin promoter (Lin et al., 2013) containing Chrimson, Chrimson-EYFP, Chrimson K176R, Chrimson-EYFP K176R, Chrimson Y261F/S267M (fChrimson), Chrimson-EYFP Y261F/S267M (f-Chrimson-EYFP), Chrimson K176R/Y261F/S267M (vf-Chrimson) and Chrimson-EYFP K176R/Y261F/S267M (vfChrimson-EYFP). The virus titer was nominally $1 \times 10^{12}-1 \times 10^{13}$ genome copies $/ \mathrm{ml}(\mathrm{GC} / \mathrm{ml})$. Briefly, $1 \times 10^{9} \mathrm{GC} / \mathrm{ml}$ of rAAV2/1 were added to each well 4-9 days after plating hippocampal neurons. Expression became visible 5 days post-transduction. The electrophysiological measurements were performed 13-21 days after transduction. No neurotoxicity was observed for the lifetime of the culture ( $\sim 5$ weeks). No all-trans retinal was added to the culture medium or recording medium for any of the experiments described here.

\section{Electrophysiological recordings on hippocampal neurons}

For whole-cell recordings in cultured hippocampal neurons, patch pipettes with resistances of 3-8 $\mathrm{M} \Omega$ were filled with $129 \mathrm{mM}$ potassium gluconate, $10 \mathrm{mM} \mathrm{HEPES}, 10 \mathrm{mM} \mathrm{KCl}, 4 \mathrm{mM}$ MgATP and 0.3 mM Na ${ }_{3} \mathrm{GTP}$, titrated to $\mathrm{pH}$ 7.2. Tyrode's solution was used as the extracellular solution (125 mM NaCl, $2 \mathrm{mM} \mathrm{KCl,} 2 \mathrm{mM} \mathrm{CaCl}_{2}, 1 \mathrm{mM} \mathrm{MgCl}_{2}, 30 \mathrm{mM}$ glucose and $25 \mathrm{mM}$ HEPES, titrated to $\mathrm{pH}$ 7.4). The series resistance was $<10 \mathrm{M} \Omega$ and the input resistance ranged from $0.7 \mathrm{G} \Omega$ to $3.5 \mathrm{G} \Omega$. The mean capacitance of the measured cells was $35.4 \pm 12.4 \mathrm{pF}(\mathrm{n}=$ 31). In order to avoid an experimental bias in cell selection, the neurons for the electrophysiological recordings were selected independent of the brightness of their EYFP fluorescence. Recordings were conducted in the presence of the excitatory synaptic transmission blockers 1,2,3,4-tetrahydro-6-nitro-2,3-dioxo-benzo[f]quinoxaline-7-sulfonamide (NBQX, 10 $\mu \mathrm{M}$, Sigma-Aldrich, St. Louis, USA) and D(-)-2-Amino-5-phosphonopentanoic acid (AP-5, 50 $\mu \mathrm{M}$, Sigma-Aldrich, St. Louis, USA). For determination of $\tau_{\mathrm{off}}$ and $\mathrm{J}_{-70 \mathrm{mV}}$ measurements were conducted in the presence of $1 \mu \mathrm{M}$ TTX (Sigma-Aldrich, St. Louis, USA) in addition. Electrophysiological signals were amplified using an Axopatch 200B amplifier (Axon Instruments, Union City, USA), filtered at $10 \mathrm{kHz}$, digitized with an Axon Digidata 1322A (50 $\mathrm{kHz}$ ) and acquired and analyzed using pClamp9 software (Axon Instruments, Union City, USA). 
The light pulses had a pulse width of $3 \mathrm{~ms}$, a wavelength of $\lambda=594 \mathrm{~nm}$ and a saturating intensity

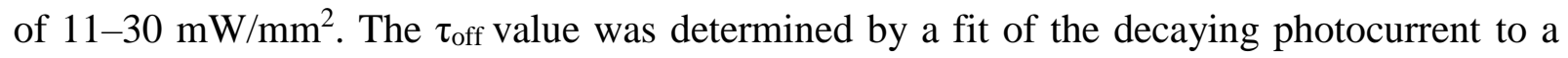
monoexponential function. The current density $\left(\mathrm{J}_{-70 \mathrm{mV}}\right)$ was determined by dividing the stationary current in response to a $500 \mathrm{~ms}$ light pulse with a saturating intensity of 20-40 $\mathrm{mW} / \mathrm{mm}^{2}$ and a wavelength of $594 \mathrm{~nm}$ by the capacitance of the cell. In order to determine the lowest light intensity required to induce action potentials with a probability of $100 \%\left(\mathrm{~J}_{100}\right), 40$ pulses $(\lambda=594 \mathrm{~nm}$, pulse width $=3 \mathrm{~ms}, v=10 \mathrm{~Hz})$ of varying light intensities were applied. The spike probability was calculated by dividing the number of light-triggered spikes by the total number of light pulses.

\section{Animals for recordings on parvalbumin-positive interneurons}

Experimental mice were obtained from a cross of PV-IRES-Cre (Hippenmeyer et al., 2005) and conditional tdTomato male and female Ai9 animals at 4-12 weeks of age (Madisen et al., 2010). Mice were maintained in a $12 \mathrm{~h}$ light/dark cycle, with access to food and water ad libitum. All animal procedures were performed in accordance with institutional guidelines and were approved by the Regierungspräsidium Darmstadt.

\section{Intracerebroventricular injections (ICVs)}

Prior to pup injections (Glascock et al., 2011), the dam was habituated to the experimenter and the experimental room. Newborn mice (P2) were anesthetized using isoflurane (2-3\%), and placed on a light source to reveal skull structures. Injections of $2 \mu$ l of AAV2/1-hSyn-vfChrimson-EYFP were performed into the right ventricle using a glass pipette (coordinates from bregma; rostral $0.75 \mathrm{~mm}$, lateral $0.25 \mathrm{~mm}$ and ventral $2 \mathrm{~mm}$ ). After injection pups were recovered for $5 \mathrm{~min}$ in a pre-warmed container with homecage-bedding before being placed back in the home cage.

\section{Patch-clamp recordings on parvalbumin-positive interneurons}

Coronal brain slices were prepared from 2-6 week-old PV-tdTomato mice that had been injected with $2 \mu \mathrm{l}$ of AAV2/1-hSyn-vf-Chrimson-EYFP at P2. Animals were anesthetized with isoflurane (3\% in oxygen), decapitated and the brain was dissected in ice-cold artificial cerebrospinal fluid (ACSF, containing in mM: $125 \mathrm{NaCl}, 3 \mathrm{KCl}, 2 \mathrm{CaCl}_{2}, 1 \mathrm{MgCl}_{2}, 26 \mathrm{NaHCO}_{3}$, 
10 glucose), and sliced (thickness: $325 \mu \mathrm{m}$ ) on a vibrating microtom (VT1200S; Wetzlar, Germany) at $4^{\circ} \mathrm{C}$. Slices were recovered for $60 \mathrm{~min}$ at room temperature in a submersion chamber containing ACSF equilibrated with $95 \%$ O2/5\% CO2. Slices were next transferred to the submersion chamber of an upright microscope (Scientifica), and continuously superfused with ACSF additionally containing $1 \mu \mathrm{M}$ DNQX, $40 \mu \mathrm{M}$ AP5 and $1 \mu \mathrm{M}$ bicuculline at $33^{\circ} \mathrm{C}$. Parvalbumin-positive interneurons were identified in layer $2 / 3$ of neocortex with a combination of infrared and fluorescence video microscopy under a 40x objective (Olympus). Patch-clamp electrodes (8-12 M 2 ) were pulled from borosilicate glass and filled with an intracellular solution consisting of (in $\mathrm{mM}$ ): 140 potassium-gluconate, $10 \mathrm{HEPES}, 4$ phosphocreatine $\mathrm{Na}_{2}, 4$ Mg-ATP, 0.4 Na-GTP, $10 \mathrm{KCl}$ (pH adjusted to 7.25 with $\mathrm{KOH}, \sim 280$ - 300mOsm). Data were acquired with a Multiclamp700B amplifier and pClamp 10.5 software (Axon Instruments). Optogenetically-evoked action potentials were recorded in parvalbumin-positive interneurons in loose-seal cell-attached $(n=4)$ or whole-cell current-clamp mode $(n=3)$. In addition, another five parvalbumin-positive interneurons were recorded for the input-output curves presented in Fig. 4.3B. Data were filtered at $20 \mathrm{kHz}$ and sampled at $50 \mathrm{kHz}$. Spiking patterns were assessed with depolarizing current steps in 8 whole-cell recordings, and displayed the fast-spiking phenotype expected for PV-interneurons $(n=8$, maximal firing frequency $301 \pm 29 \mathrm{~Hz})$. Optical stimulation was performed through the objective by an LED (coolLED) coupled to the microscope. Pulse width was $0.25-1 \mathrm{~ms}$, irradiance ranged from $1-10 \mathrm{~mW} / \mathrm{mm}^{2}$, and was adjusted individually for every neuron to cause reliable firing (Figure 4.3C: $50 \mathrm{~Hz}$, pulsewidth $=0.5 \mathrm{~ms}$, light-density $=2.6 \mathrm{~mW} / \mathrm{mm}^{2} ; 100 \mathrm{~Hz}$, pulsewidth $=0.5 \mathrm{~ms}$, light-density $=2.6$ $\mathrm{mW} / \mathrm{mm}^{2} ; 150 \mathrm{~Hz}$, pulsewidth $=0.5 \mathrm{~ms}$, light-density $=4.9 \mathrm{~mW} / \mathrm{mm}^{2} ; 200 \mathrm{~Hz}$, pulsewidth $=$ $0.5 \mathrm{~ms}$, light-density $=4.9 \mathrm{~mW} / \mathrm{mm}^{2} ; 250 \mathrm{~Hz}$, pulsewidth $=0.5 \mathrm{~ms}$, light-density $=4.9$ $\mathrm{mW} / \mathrm{mm}^{2} ; 300 \mathrm{~Hz}$, pulsewidth $=0.5 \mathrm{~ms}$, light-density $=4.9 \mathrm{~mW} / \mathrm{mm}^{2} ; 400 \mathrm{~Hz}$, pulsewidth $=$ $0.5 \mathrm{~ms}$, light-density $=4.9 \mathrm{~mW} / \mathrm{mm}^{2} ; 500 \mathrm{~Hz}$, pulsewidth $=0.5 \mathrm{~ms}$, light-density $=8 \mathrm{~mW} / \mathrm{mm}^{2}$ ). Data were analyzed using Clampfit and Excel software. For calculation of latency and jitter, the time of action potential peak was used. We note that the apparent action potential threshold defined as the voltage at which the first temporal derivative crosses a threshold of $40 \mathrm{~V} / \mathrm{s}$ is more hyperpolarized for optogentically evoked action potentials $(-57.00 \pm 1.85 \mathrm{mV})$ compared to action potentials during DC current injections $(-43.19 \pm 2.97 \mathrm{mV}, p<0.001$, unpaired, twotailed t-test). Statistics were done using the non-parametric Friedmann test followed by the posthoc Dunn's test (Prism, GraphPad Sofware Inc., La Jolla, USA). 


\section{Cloning for AAV2/6 production}

pcDNA3.1(-)_f-Chrimson_EYFP was used as a starting material for cloning pAAV_hSyn_fChrimson_EYFP. The sequence of f-Chrimson_EYFP was amplified by means of a classical PCR. The resulting PCR fragment was then digested with BamHI/HindIII (Thermo Scientific, MA, USA), gel extracted (GeneJET Gel Extraction Kit, Thermo Scientific, MA, USA) and further used for ligation. At the same time the plasmid pAAV_hSyn_Chronos_GFP (Addgene, plasmid nr. 59170) was also digested using restriction enzymes BamHI/HindIII and used as a backbone plasmid. All obtained ligation products were further tested by means of colony PCR and finally sequenced by an external company. The final product was then sent to the University of North Carolina Vector Core (Chapel Hill, NC, USA), and used to produce AAV2/6.

\section{Postnatal AAV injection into the cochlea}

All experiments were done in compliance with the German national animal care guidelines and were approved by the board for animal welfare of the University Medical Center Göttingen and the animal welfare office of the state of Lower Saxony. The calculation of animal number was performed prior to starting experiments. We planned to use the Wilcoxon Rank Sum Test and an error probability alpha smaller than 0.05 , a power (1-beta) of 0.95 and effect size depending on the precise experimental protocol.

Postnatal AAV-injection into scala tympani of the left ear via the round window (Akil et al., 2012) was performed at P3-P6 on C57BL/6 wild-type mice, using AAV2/6 and the human synapsin promoter to drive transgenic expression of f-Chrimson-EYFP in SGNs. In brief, under general isoflurane anaesthesia and local analgesia achieved by means of xylocaine, the left ear was approached via a dorsal incision and the round window membrane was identified and gently punctured using a borosilicate capillary pipette that was kept in place to inject approximately 5 $\mathrm{x} 10^{9}$ viral genomes. After virus application, the tissue above the injection site was repositioned, the wound was sutured and buprenorphine $(0.1 \mathrm{mg} / \mathrm{kg})$ was applied as pain reliever. Recovery of the animals was then daily tracked. Mice were randomly selected for injection in all experiments. No blinding was possible since injections had to be performed in the left ear leaving the right ear as an internal control. Hence, surgery prior to stimulation needed to be done in the injected ear. Animals were then kept in a $12 \mathrm{~h}$ light/dark cycle, with access to food and water ad libitum. 


\section{Immunostaining and imaging of cochlear cryosections}

Cochleae were fixed with $4 \%$ paraformaldehyde in phosphate buffered saline for $1 \mathrm{~h}$. Cochleae were then cryosectioned following $0.12 \mathrm{M}$ EDTA decalcification. After incubation of sections for $1 \mathrm{~h}$ in goat serum dilution buffer ( $16 \%$ normal goat serum, $450 \mathrm{mM} \mathrm{NaCl}, 0.6 \%$ Triton $\mathrm{X}$ 100, $20 \mathrm{mM}$ phosphate buffer, $\mathrm{pH} 7.4$ ), primary antibodies were applied for $1 \mathrm{~h}$ at room temperature. The following antibodies were used: chicken anti-GFP (catalog number: ab13970, dilution 1:500) (Abcam, Cambridge, United Kingdom), guinea pig anti-parvalbumin (catalog number: 195004, dilution 1:300) (Synaptic Systems, Göttingen, Germany). The following secondary AlexaFluor-labeled antibodies were applied for $1 \mathrm{~h}$ at room temperature: goat antichicken 488 IgG $(\mathrm{H}+\mathrm{L})$, catalog number: A-11039, dilution 1:200 (Thermo Scientific, MA, USA); goat-anti guinea pig 568 IgG (H+L), catalog number A1107, dilution 1:200 (Thermo Scientific, MA, USA).

Confocal images were collected using a SP5 microscope (Leica, Hamburg, Germany) and processed in Image J (NIH, Bethesda, MD, USA). Expression was considered positive when EYFP fluorescence in a given cell (marked by parvalbumin) was found to be higher than 3 s.d. above the background fluorescence of the tissue.

\section{Animal surgery for recordings on the auditory pathway}

Mice were anesthetized with intraperitoneal administration of a mixture of xylazine $(5 \mathrm{mg} / \mathrm{kg})$ and urethane $(1.32 \mathrm{mg} / \mathrm{kg})$ while analgesia was achieved with buprenorphine. The core temperature was maintained constant at $37^{\circ} \mathrm{C}$ using a custom-designed heat plate on a vibration isolation table in a sound-proof chamber (IAC GmbH, Niederkrüchten, Germany). For auditory nerve recordings, a tracheostomy was performed before the animals were positioned in a custom-designed stereotactic head holder. Pinnae were removed, scalp reflected, portions of the lateral interparietal and of the left occipital bone removed, and a partial cerebellar aspiration performed to expose the surface of the cochlear nucleus.

\section{Optical stimulation in vivo}

The left bulla was reached using a retroauricular approach and opened to expose the cochlea. A $50 \mu \mathrm{m}$ optical fiber coupled to a 594-nm laser (OBIS LS OPSL, $100 \mathrm{~mW}$, Coherent Inc., Santa 
Clara, CA, USA) was inserted into the cochlea via the round window. Radiant flux was calibrated with a laser power meter (LaserCheck; Coherent Inc., Santa Clara, CA, USA).

\section{SGN culture and patch-clamp recordings}

On P12-14, SGNs of injected mice were isolated, cultured and patch-clamped (Smith et al., 2015). In brief, we patch-clamped EYFP-positive SGNs using an EPC-10 amplifier controlled by Patchmaster software (HEKA electronics, Lambrecht, Germany) and employing potassiumgluconate based intracellular solution (in mM: $130 \mathrm{~K}$-gluconate, $5 \mathrm{KCl}, 1$ EGTA, $2 \mathrm{MgATP}, 2$ $\mathrm{Na}_{2} \mathrm{ATP}, 0.3 \mathrm{MgGTP}, 10 \mathrm{KOH}-\mathrm{HEPES}, 10 \mathrm{Na}_{2}$ Phosphocreatinine) and an extracellular solution containing (in mM: $145 \mathrm{NaCl}, 4 \mathrm{KCl}, 1 \mathrm{MgCl}_{2}, 1.3 \mathrm{CaCl}, 10 \mathrm{NaOH}-\mathrm{HEPES}$. A 594-nm laser (OBIS LS OPSL, 100 mW, Coherent Inc., Santa Clara, CA, USA) was coupled into a Nikon Eclipse inverted microscope and radiant flux was calibrated using a powermeter.

\section{Auditory brainstem responses}

For stimulus generation and presentation, data acquisition, and off-line analysis, we used a NI System (National Instruments, Austin, TX, USA) and custom-written MATLAB software (The MathWorks, Inc., Natick, MA, USA). Optically-evoked ABRs (oABRs) and acousticallyevoked ABRs (aABRs) were recorded by needle electrodes underneath the pinna, on the vertex, and on the back near the legs. The difference potential between vertex and mastoid subdermal needles was amplified using a custom-designed amplifier, sampled at a rate of $50 \mathrm{kHz}$ for 20 ms, filtered (300-3000 Hz) and averaged across 1000 and 500 presentations (for oABRs and aABRs, respectively). Thresholds were determined by visual inspection as the minimum sound or light intensity that elicited a reproducible response waveform in the recorded traces.

\section{Juxtacellular recordings from single putative SGNs}

For auditory nerve recordings (Hernandez et al., 2014b), a glass microelectrode ( 25 M $\Omega$ ) was advanced through the posterior end of the anteroventral cochlear nucleus, aiming toward the internal auditory canal using an Inchworm micropositioner (EXFO Burleigh). Extracellular action potentials were amplified using an ELC-03XS amplifier (NPI Electronic, Tamm, Germany), filtered (band pass, 300-3000 Hz), and digitized (TDT System 3) using customwritten Matlab (Mathworks) software. Data were further analyzed and prepared for display offline using custom-written Python (Python software foundation, Delaware, USA) and Matlab 
software. Once light-responsive fibers were encountered, stimulation was performed by means of 400 or 900 ms-long light-pulse trains at varying stimulation rates, leaving $100 \mathrm{~ms}$ inter-train recovery over 20 repetitions. Responses within the first $400 \mathrm{~ms}$ were then used for analysis. Only recordings for which the fibers generate at least 5 spikes per light-pulse train (on average across the 20 iterations recorded for each frequency tested on each fiber) were included. Phaselocking was quantified using the vector strength (Goldberg and Brown, 1969) and its significance tested with the Rayleigh test. If $\mathrm{L}>13.8$, the null hypothesis was rejected at the 0.001 significance level (Hillery and Narins, 1987): insignificant VS were set to 0. The temporal jitter, defined as the standard deviation of spike latency across trials, was calculated using a time window equal to the stimulation period. The hazard function of the temporal jitter was evaluated for each stimulation rate by simulating Poisson spike trains at discharge rates from 10 to 300 spikes/s. The spike probability is the ratio between the number of spikes and the number of light-pulses.

\section{Data analysis}

The data were analyzed using Matlab (The MathWorks, Inc., Natick, MA, USA), Excel, Igor Pro 6 (Wavemetrics, Portland, OR, USA), Origin 9.0 (OriginLab, Inc., Northampton, MA, USA), and GraphPad Prism (GraphPad Software, La Jolla, CA, USA). Averages were expressed as mean \pm s.e.m. or mean \pm s.d., as specified. References to data in the main text were expressed as mean \pm s.e.m. For statistical comparison between two groups, data sets were tested for normal distribution (using the D'Agostino \& Pearson omnibus normality test or the Shapiro-Wilk test) and equality of variances (F-test) followed by two-tailed unpaired Student's t-test, or the unpaired two-tailed Mann-Whitney $U$ test when data were not normally distributed and/or variance was unequal between samples.

For evaluation of multiple groups, statistical significance was calculated by using one-way ANOVA test followed by Tukey's test for normally distributed data (equality of variances tested with the Brown-Forsythe test) or one-way Kruskal-Wallis test followed by Dunn's test for nonnormally distributed data. 


\section{Data availability}

The data that support the findings of this study and code used for analysis are available from the corresponding author upon reasonable request.

Acknowledgements: We would like to thank Nicola Strenzke for co-supervising the work of David Lopez de la Morena. We would like to thank Ina Bartnik and Nicole Fürst for the preparation of the rat hippocampal neuron cultures. We also would like to thank Verena Pintschovius, Heike Fotis, Sandra Gerke and Christiane Senger-Freitag for excellent technical assistance, Helga Husmann for help with the preparation of the figures and Gerhard Hoch for programming stimulation software. The Chrimson clone was kindly provided by Edward S. Boyden. This work was supported by the German Research Foundation Collaborative Research Centers 807 (to E.B.) and 889 (to To.M)., Center of Excellence Frankfurt Macromolecular Complexes (to E.B.), the Center for Nanoscale Microscopy and Molecular Physiology of the Brain (to To.M.), by the Max Planck Society (to E.B. and J.L.), by the European Research Council (ERC advanced grant "OptoHear" to To.M. and ERC starting grant "AttentionCircuits" to J.L.).

Author Contributions: T.Ma., P.G.W., J.J.L., T.Mo. and E.B designed research; T.Ma. (Fig. 4.1, Fig. 4.2, Table 4.1, Fig. S1-S6, Table S1-S4), D.L.M. (Fig. 4.4-4.6, Fig. S9-S10), V.S. (Fig. 4.3, Fig. S8), J.S. (Table 4.1, Fig. S1, Table S1, Table S2), A.D. (Fig. 4.2, Fig. S7, Table S4), K.F. (Table S1), C.W. (Fig. 4.5), S.J. (Fig. 4.4), K.B (Fig. 4.4 and 4.5), VR (Fig. 4.4), LB (Fig. 4.4) and A.H. (Fig. 4.6 and Fig. S10) performed research and analyzed data; J.J. prepared the AAVs; T.Ma., D.L.M., T.Mo. and E.B with contributions from V.S. and J.J.L. wrote paper.

Competing financial interest: The authors declare no competing financial interests. 


\section{Supplementary Information for}

\section{High frequency neural spiking and auditory signaling by ultrafast red- shifted optogenetics}
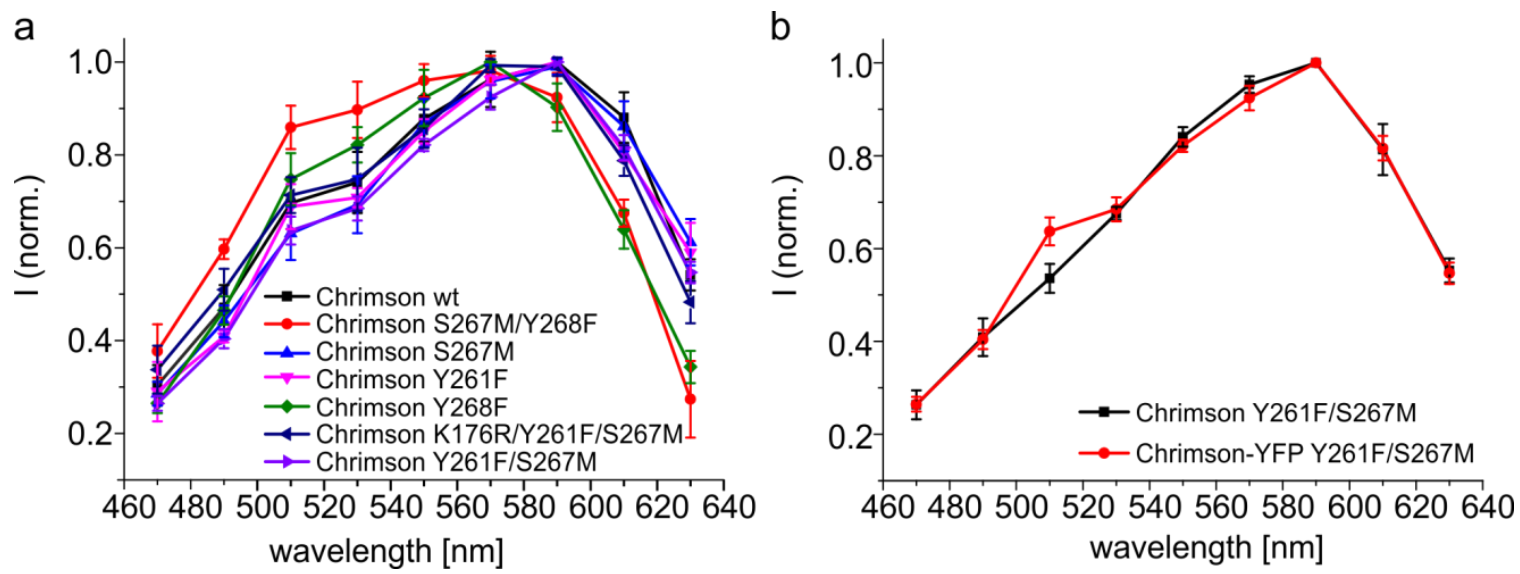

Supplementary Figure 1. Action spectra of Chrimson and Chrimson mutants. Shown are normalized peak currents in response to ns light-pulses of indicated wavelength. The electrophysiological recordings were performed in NG cells by patch-clamp experiments in the whole-cell configuration at a membrane potential of $-60 \mathrm{mV}$. (a) Action spectra of ChrimsonEYFP (־, n=6), Chrimson-EYFP S267M/Y268F ( - , n=3), Chrimson-EYFP S267M ( $\smile$, n=3), Chrimson-EYFP Y261F ( $\multimap, n=4)$, Chrimson-EYFP Y268F $(\neg, \mathrm{n}=5)$, Chrimson-EYFP K176R/Y261F/S267M (vf-Chrimson-EYFP) (־, n=4) and ChrimsonEYFP Y261F/S267M (f-Chrimson-EYFP) ( $\rightarrow$, n=4). (b) Action spectra of Chrimson Y261F/S267M (f-Chrimson) ( $\rightarrow, n=3$ ) and Chrimson-EYFP Y261F/S267M (f-ChrimsonEYFP) $(-\cdots, n=4)$. The pulse energies at the different wavelengths were set to equal photon counts for the recordings of the action spectra. Experimental details are described in the Methods section. 


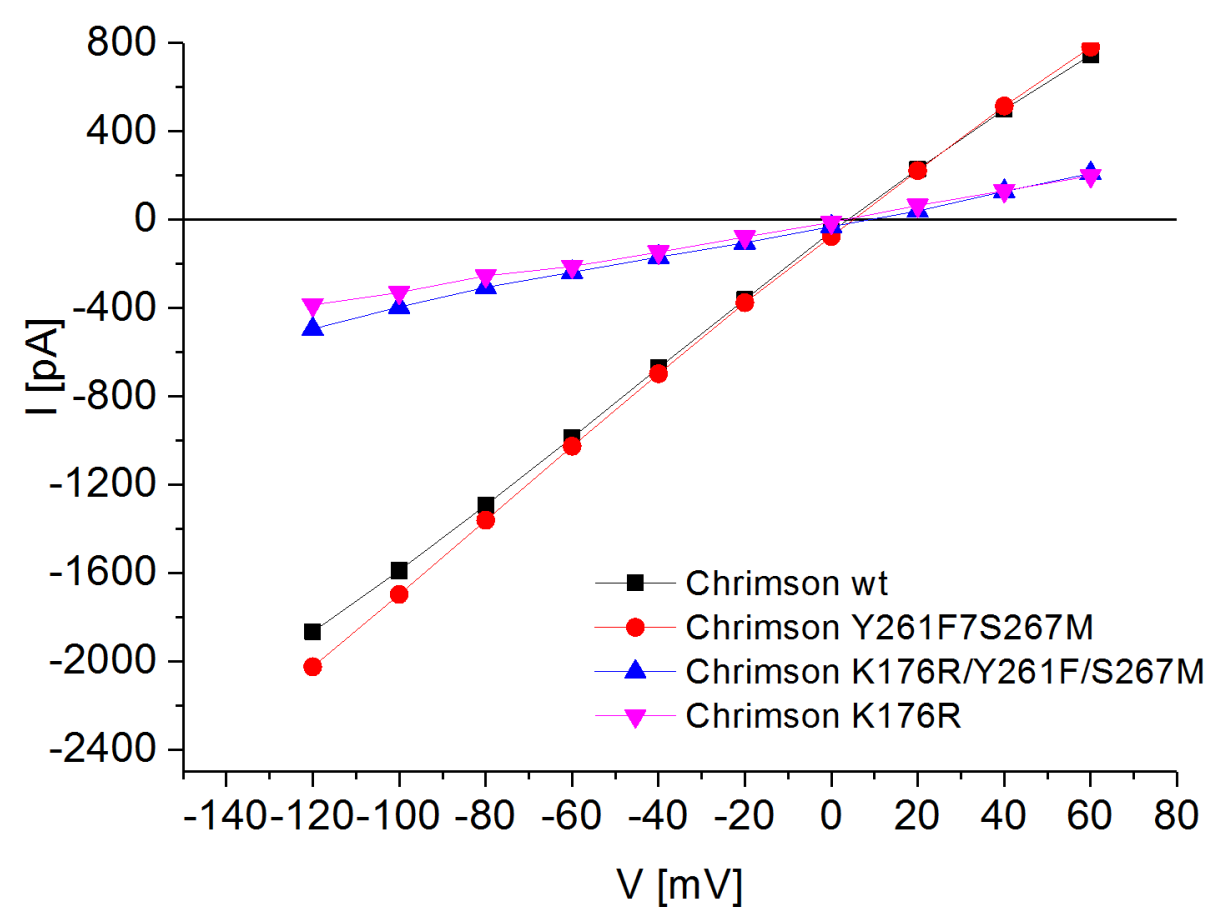

Supplementary Figure 2. Current (I) - voltage (V) curves of Chrimson and Chrimson mutants. Shown are typical IV curves of Chrimon-EYFP wt, Chrimson-EYFP Y261F/S267M (fChrimson-EYFP), Chrimson-EYFP K176R/Y261F/S267M (vf-Chrimson-EYFP) and Chrimson-EYFP K176R recorded in NG cells. Whole-cell patch-clamp experiments were performed under voltage-clamp conditions. The photocurrents were measured in response to 3 ms light-pulses with a wavelength of $594 \mathrm{~nm}$ and a saturating light intensity of $23 \mathrm{~mW} / \mathrm{mm}^{2}$. Peak currents were taken for the quantification. 


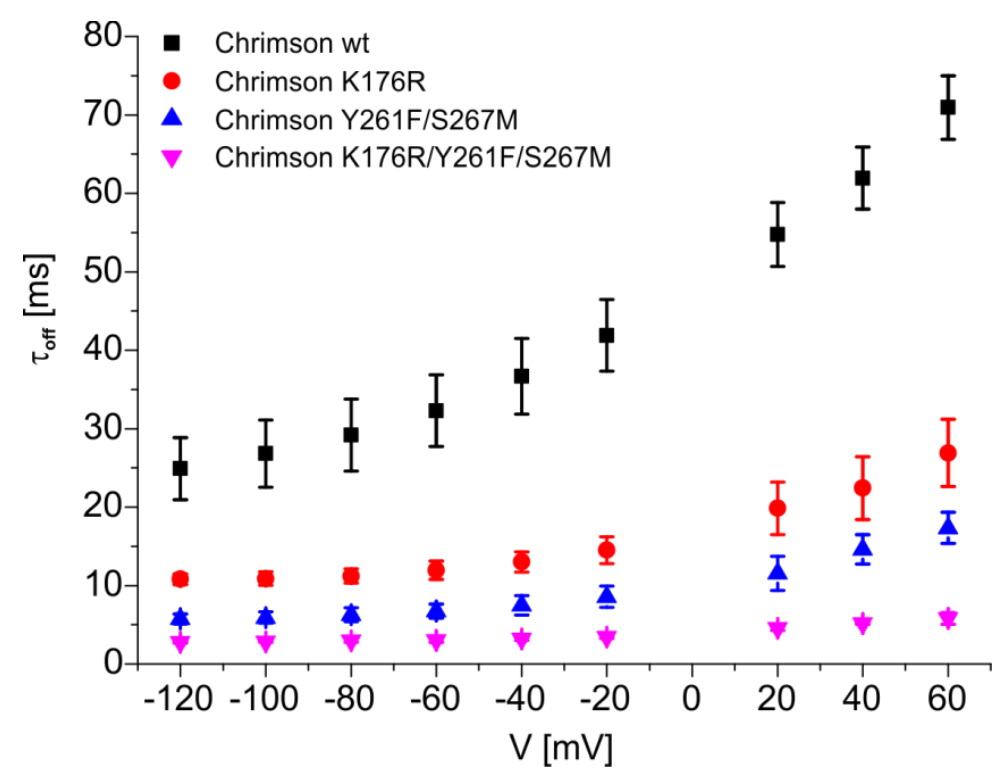

Supplementary Figure 3. Off-kinetics of Chrimson and Chrimson mutants at different membrane potentials. Whole-cell patch-clamp experiments were performed under voltageclamp conditions. Photocurrents were measured in response to $3 \mathrm{~ms}$ light pulses with a saturating intensity of $23 \mathrm{~mW} / \mathrm{mm}^{2}$ and a wavelength of $594 \mathrm{~nm}$. Shown is the average $\tau_{\text {off }}$ for Chrimson-EYFP (a, n=3), Chrimson-EYFP K176R (•, n=4), Chrimson-EYFP Y261F/S267M (f-Chrimson-EYFP) ( $\Delta, \mathrm{n}=3)$ and Chrimson-EYFP K176R/Y261F/S267M (vf-ChrimsonEYFP $(\nabla, n=3)$. The error bars represent the standard deviation. The $\tau_{\text {off }}$ values were determined as described in the Methods section. 


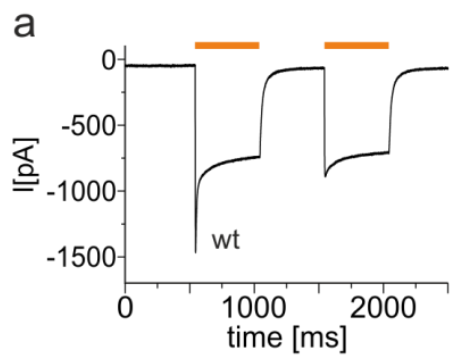

d
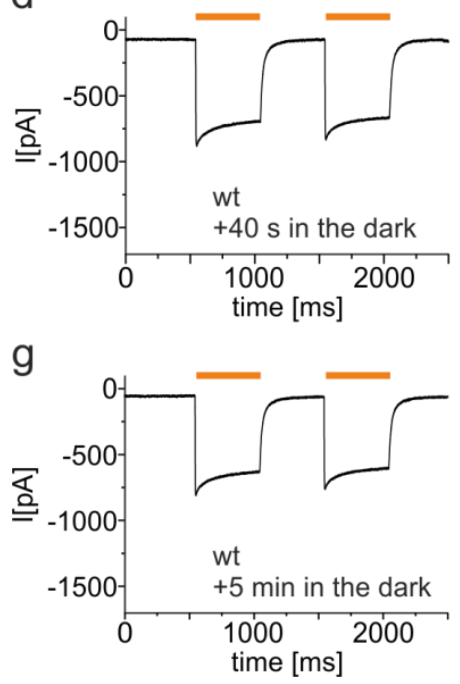

b

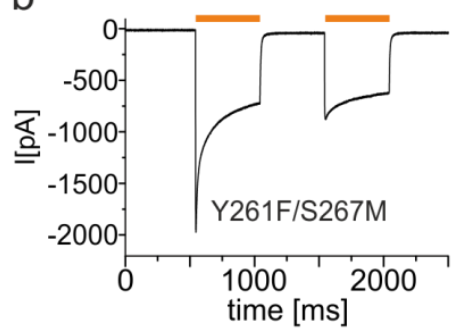

e

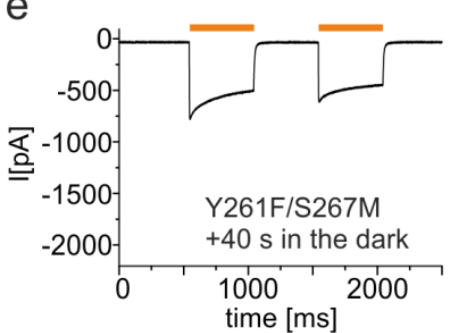

h

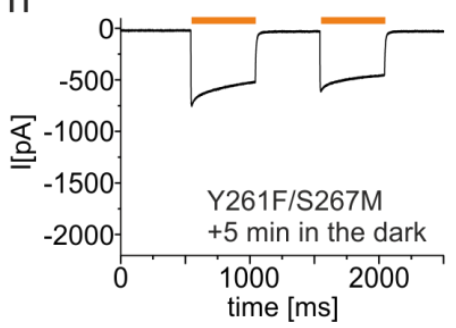

C

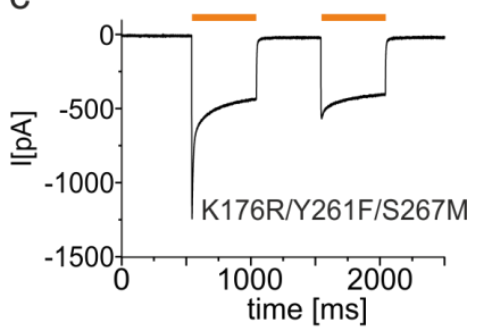

f

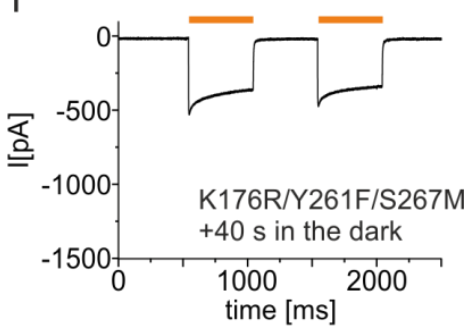

i

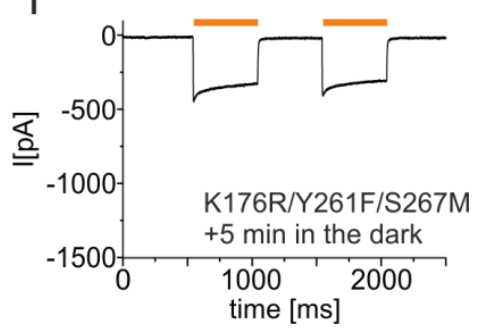

Supplementary Figure 4. Peak current inactivation and recovery of Chrimson and Chrimson mutants. Typical photocurrents in response to a double illumination protocol using $500 \mathrm{~ms}$ light pulses with a saturating intensity of $23 \mathrm{~mW} / \mathrm{mm}^{2}$ and a wavelength of $594 \mathrm{~nm}$. The photocurrents of Chrimson-EYFP (a,d,g), Chrimson-EYFP Y261F/S267M (f-Chrimson-EYFP) (b,e,h) and Chrimson-EYFP K176R/Y261F/S267M (vf-Chrimson-EYFP) (c,f,i) were measured by patch-clamp experiments in the whole-cell configuration at a membrane potential of $-60 \mathrm{mV}$. The double illumination protocol was repeated after a waiting time of $40 \mathrm{~s}(\mathbf{d}, \mathbf{e , f})$ and 5 min $(\mathbf{g}, \mathbf{h}, \mathbf{i})$ in the dark. 

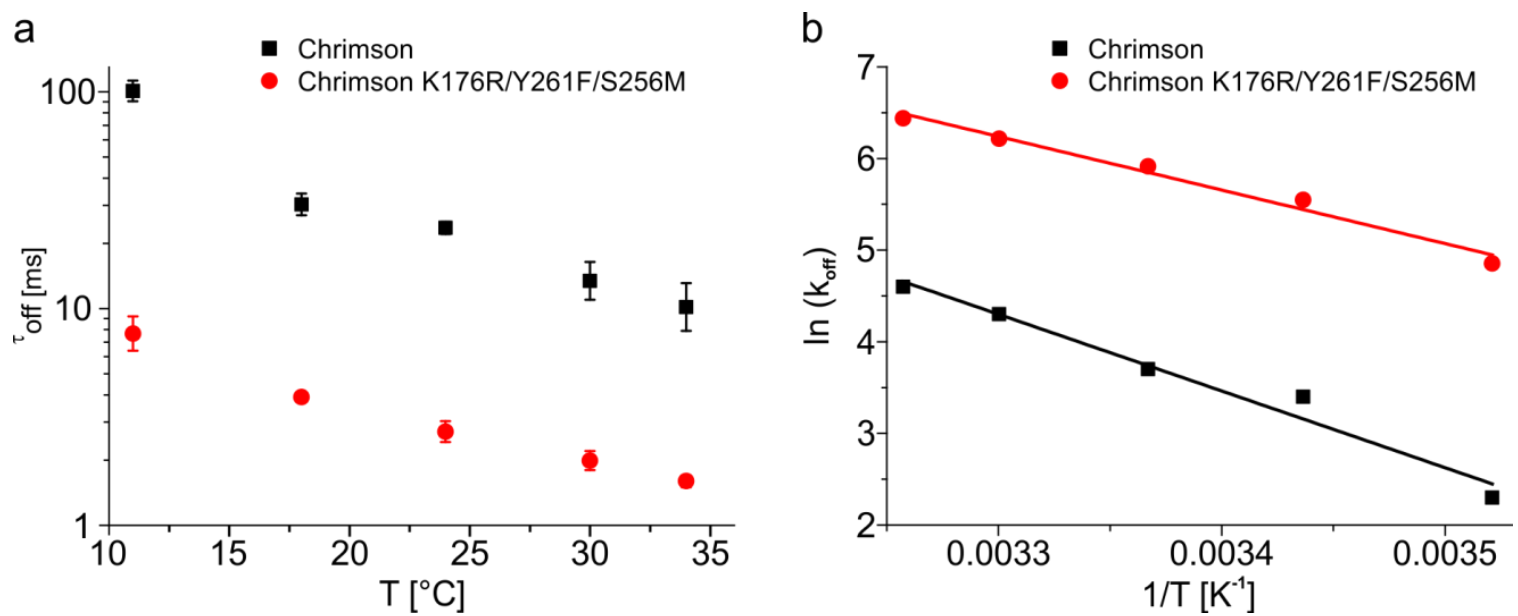

Supplementary Figure 5. Temperature dependence of the off-kinetics of Chrimson and Chrimson K176R/Y261F/S267M (vf-Chrimson). (a) Shown are the average $\tau_{\text {off }}$ values $(n=3-7)$ and the corresponding standard deviations. NG cells transiently expressing Chrimson-EYFP and Chrimson-EYFP K176R/Y261F/S267M were investigated by patch-clamp measurements in the whole-cell configuration at a membrane potential of $-60 \mathrm{mV}$. All photocurrents, but the photocurrents recorded at $34^{\circ} \mathrm{C}$, were measured in response to $3 \mathrm{~ms}$ light pulses with an intensity of $23 \mathrm{~mW} / \mathrm{mm}^{2}$ and a wavelength of $594 \mathrm{~nm}$. The photocurrents recorded at a temperature of $34^{\circ} \mathrm{C}$ were measured in response to light-pulses with a pulse length of $7 \mathrm{~ns}$ and a wavelength of $594 \mathrm{~nm}$, which were generated with the Opolette 355 tunable laser system (Opotek Inc). Thereby the pulse energy was set to value of $10^{19}$ photons $/ \mathrm{m}^{2}$. The $\tau_{\text {off }}$ values were determined by a fit of the decaying photocurrents to a monoexponential function. (b) Arrhenius plot of the data shown in A. The lines indicate activation energies of $69.6 \mathrm{~kJ} / \mathrm{mol}$ for Chrimson and $48.5 \mathrm{~kJ} / \mathrm{mol}$ for Chrimson K176R/Y261F/S267M. 
a)

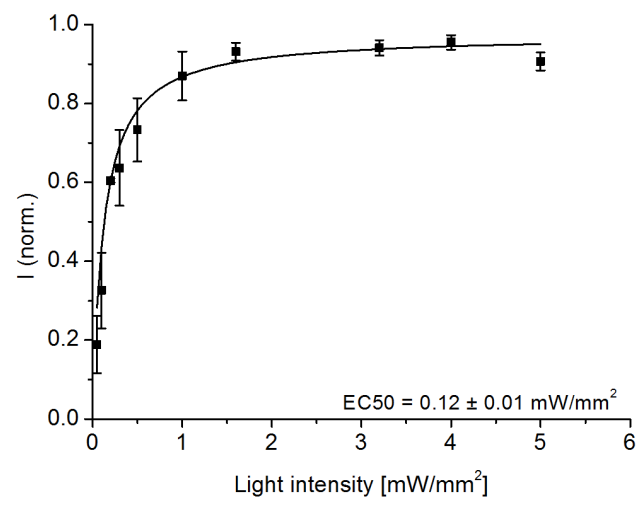

c)

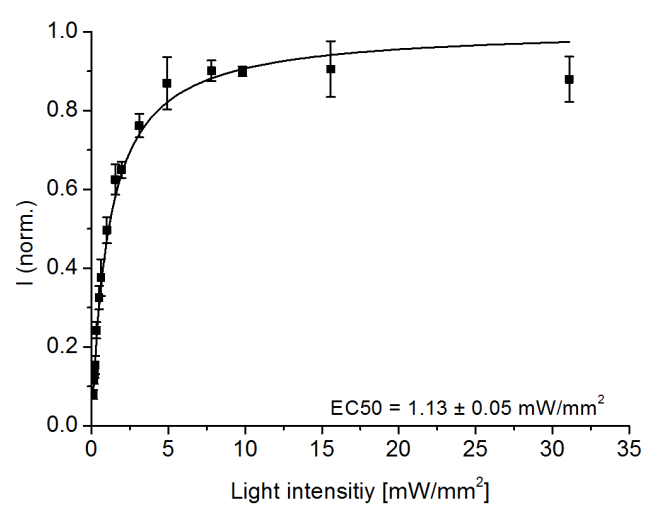

e)

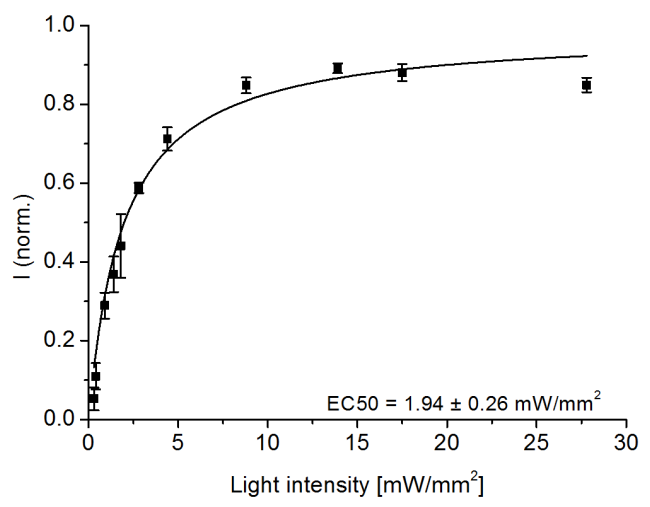

b)

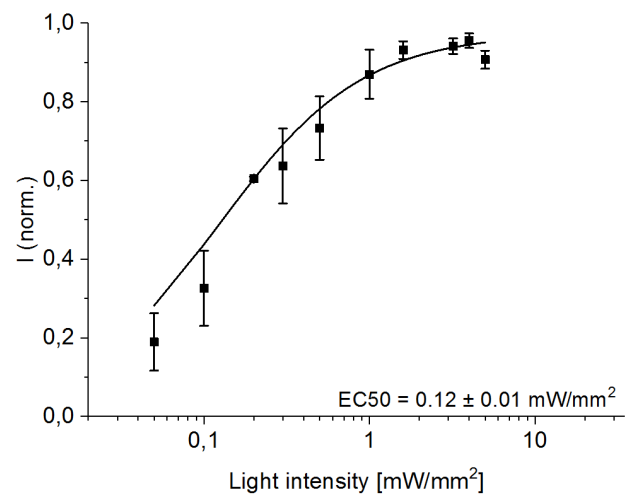

d)

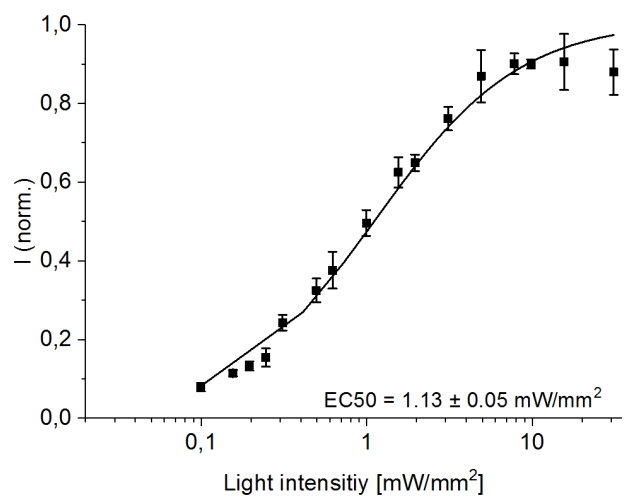

f)

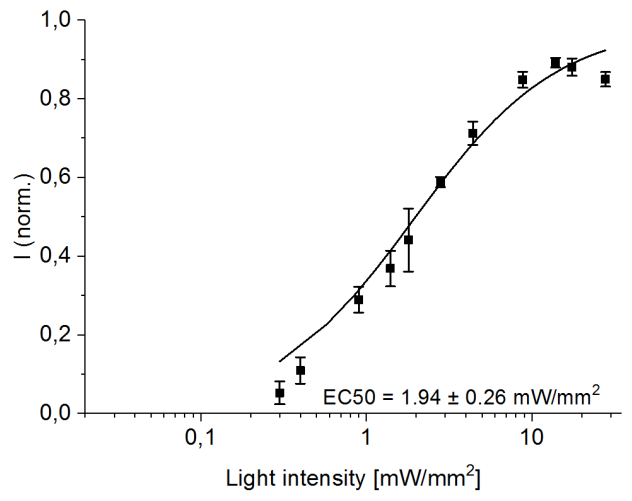

Supplementary Figure 6. Light intensity dependence of Chrimson and Chrimson mutants. Chrimson-EYFP (a,b), f-Chrimson-EYFP (c,d) and vf-Chrimson-EYFP (e,f) were investigated in NG108-15 cells by patch-clamp experiments in the whole-cell configuration at a membrane potential of $-60 \mathrm{mV}$. The photocurrents were measured in response to $500 \mathrm{~ms}$ light-pulses of indicated light intensities $(\lambda=594 \mathrm{~nm})$. The graphs show average values from normed stationary photocurrents $(n=3)$ and the corresponding standard deviations. The solid line is a fit to the data using a hyperbolic model function with a half-saturating value EC50 and a fixed $v_{\max }=1$. The light intensity is shown in linear scale $(\mathbf{a}, \mathbf{c}, \mathbf{e})$ and in logarithmic scale (b,d,f). 

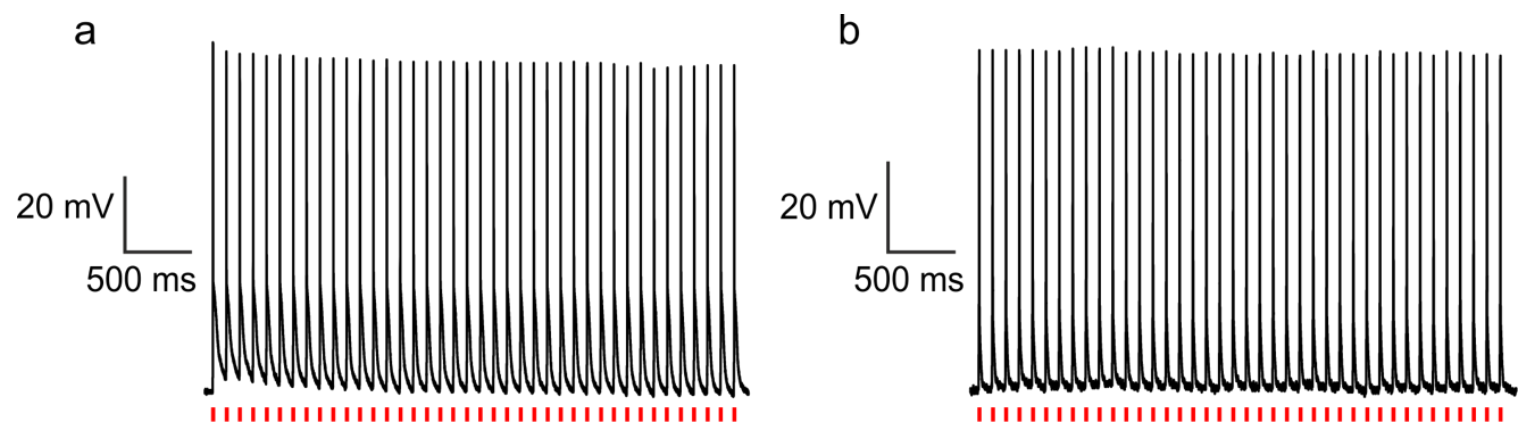

Supplementary Figure 7. Red light triggered spiking in rat hippocampal neurons. Rat hippocampal neurons heterologously expressing f-Chrimson-EYFP (a) and vf-Chrimson-EYFP (b) were investigated by patch-clamp experiments in the whole-cell configuration under currentclamp conditions. The action potentials were triggered by 40 light-pulses $(v=10 \mathrm{~Hz})$ with a pulse width of $3 \mathrm{~ms}$, a wavelength of $640 \mathrm{~nm}$ and a saturating light intensity of $23 \mathrm{~mW} / \mathrm{mm}^{2}$. 

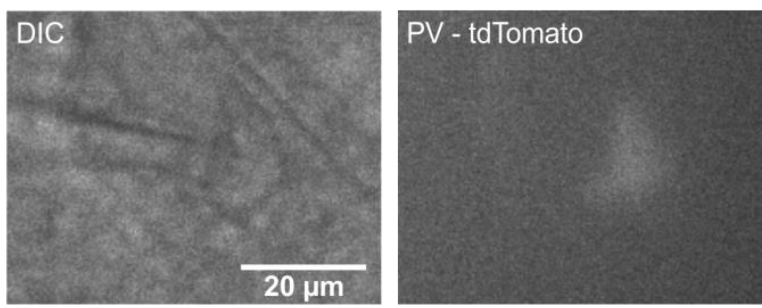

b
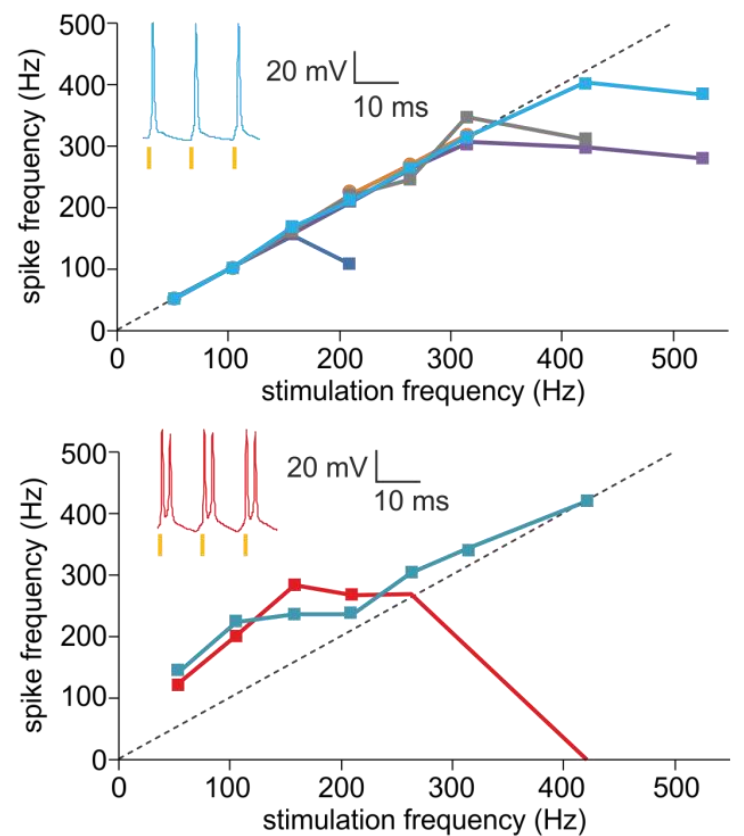
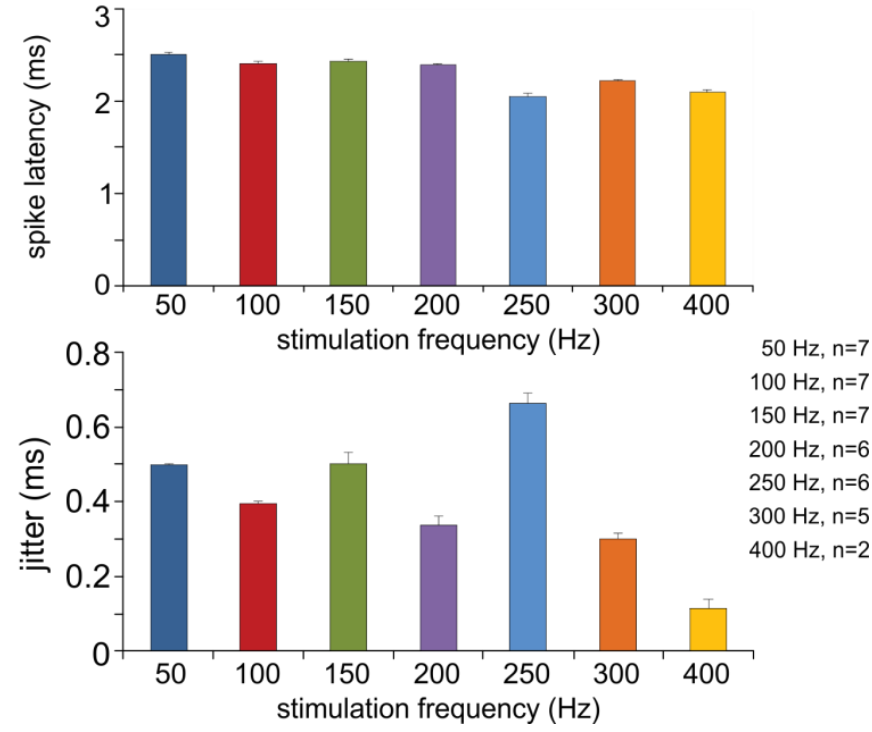

Supplementary Figure 8. Fidelity and temporal precision of light triggered spiking in parvalbumin-positive interneurons heterologously expressing vf-Chrimson-EYFP. (a) Example IR-DIC and fluorescent image of a recorded neocortical parvalbumin-positive interneuron identified by red fluorescence in an acute slice. (b) Action potential frequency as a function of stimulation frequency. Most neurons followed optical stimulation faithfully with 1 action potential/light pulse up to a maximum, and then plateaued off (top, $n=5$ ). In 2 interneurons, single light pulses could also elicit action potential doublets (bottom, $n=2$ ). We note that optimization of expression time and stimulation light density is expected to minimize this caveat. Insets: example traces of cells shown in blue and red at $100 \mathrm{~Hz}$ stimulation frequency. (c) Upper panel: action potential latency (assessed at peak) after light pulse onset for all stimulation frequencies with reliable spiking (> 85\%). (non-parametric Friedman test with a post hoc Dunn's multiple comparison test revealed the following significant differences: $50 \mathrm{~Hz}$ vs $250 \mathrm{~Hz}$, ***; $50 \mathrm{~Hz}$ vs $300 \mathrm{~Hz}$, ***; $100 \mathrm{~Hz}$ vs $250 \mathrm{~Hz}$, ***; $100 \mathrm{~Hz}$ vs $300 \mathrm{~Hz} * ; 150 \mathrm{~Hz}$ vs $250 \mathrm{~Hz}, * * * ; 150 \mathrm{~Hz}$ vs $300 \mathrm{~Hz}, * * ; 200 \mathrm{~Hz}$ vs $250 \mathrm{~Hz}$, ***.) Lower panel: action potential jitter (s.d. of latencies) for the different stimulation frequencies (average of 20 spikes/trace and 10 traces). (non-parametric Friedman test with post hoc Dunn's multiple comparison test revealed the following significant differences: $50 \mathrm{~Hz}$ vs $200 \mathrm{~Hz}$, ***; $50 \mathrm{~Hz}$ vs $300 \mathrm{~Hz}$, ***; $100 \mathrm{~Hz}$ vs $250 \mathrm{~Hz}$, ***; $150 \mathrm{~Hz}$ vs $200 \mathrm{~Hz}$, *; $150 \mathrm{~Hz}$ vs $300 \mathrm{~Hz}$, ***; $200 \mathrm{~Hz}$ vs $250 \mathrm{~Hz}$, ***; $250 \mathrm{~Hz}$ vs $300 \mathrm{~Hz}$, $* * *$.). Error bars are s.e.m; $p \leq 0.05=*, p \leq 0.01=* *, p \leq 0.001=* * *$. 


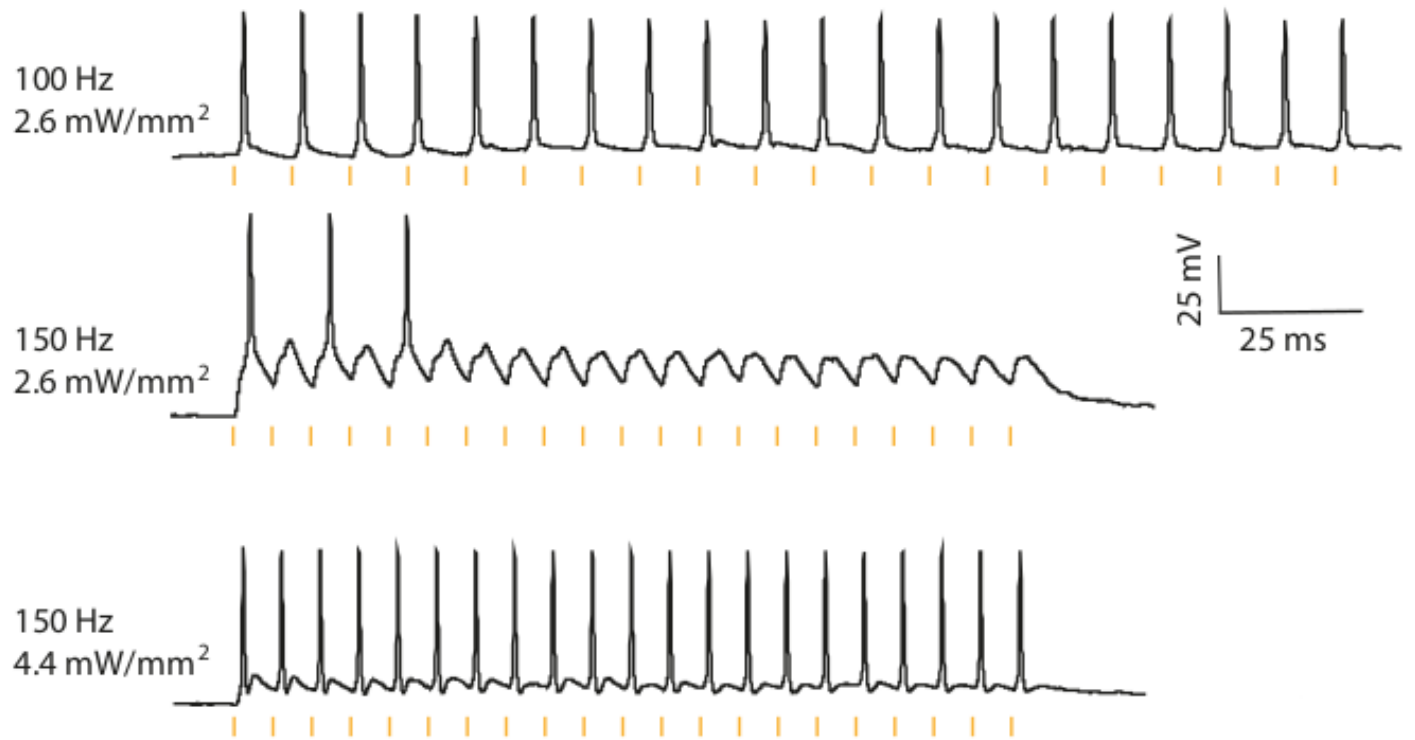

Supplementary Figure 9. Precise optogenetic control of parvalbumin-positive interneurons depends on irradiance and stimulation frequency. Exemplary traces showing precise spiking at $100 \mathrm{~Hz}$ stimulation frequency, but stimulation failures at $150 \mathrm{~Hz}$ for the same irradiance level $\left(2.6 \mathrm{~mW} / \mathrm{mm}^{2}\right)$. Increasing the light intensity restored precise spiking (bottom). 


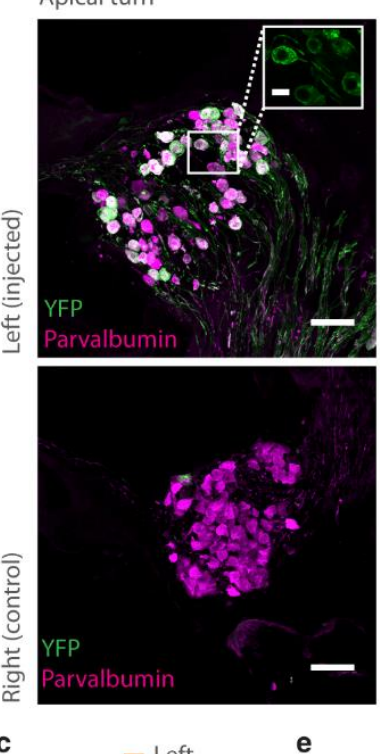

Mid turn
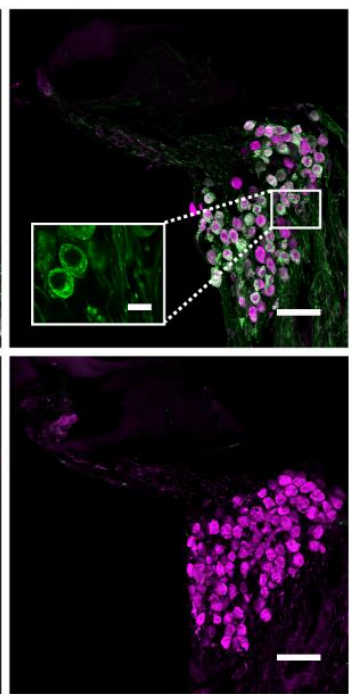

c

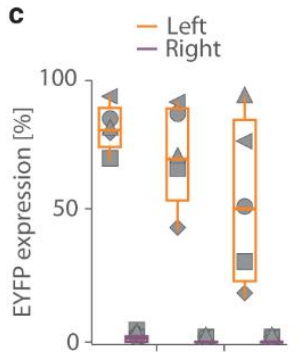

d

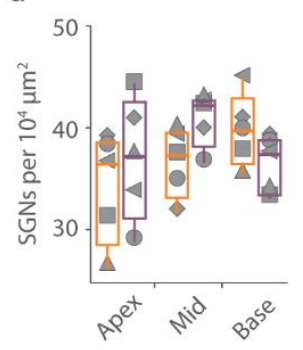

h
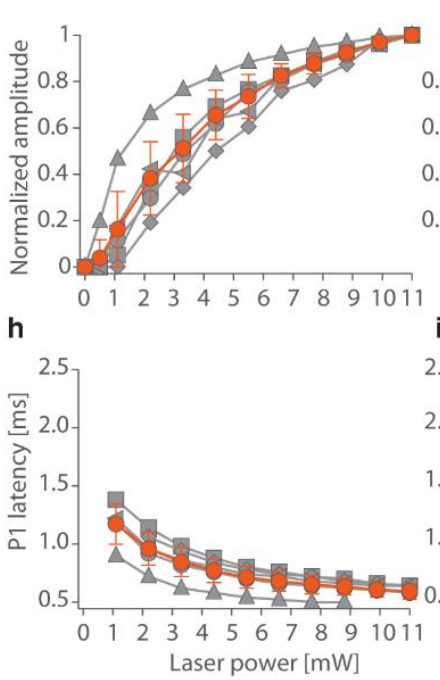

Basal turn
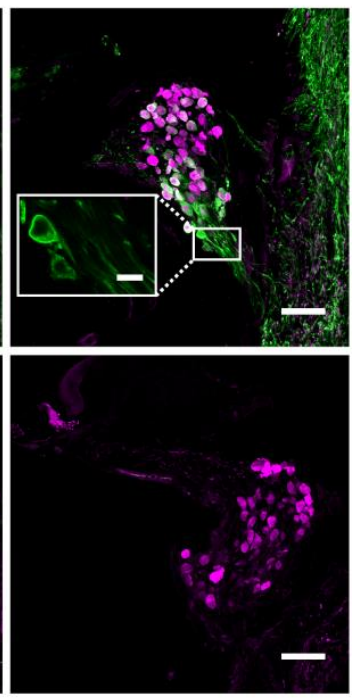

f

b
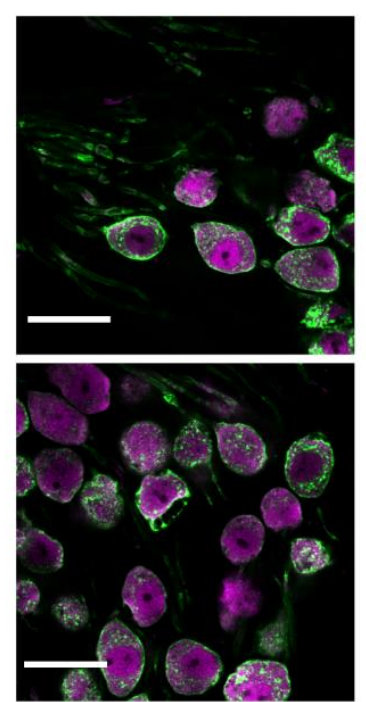

g
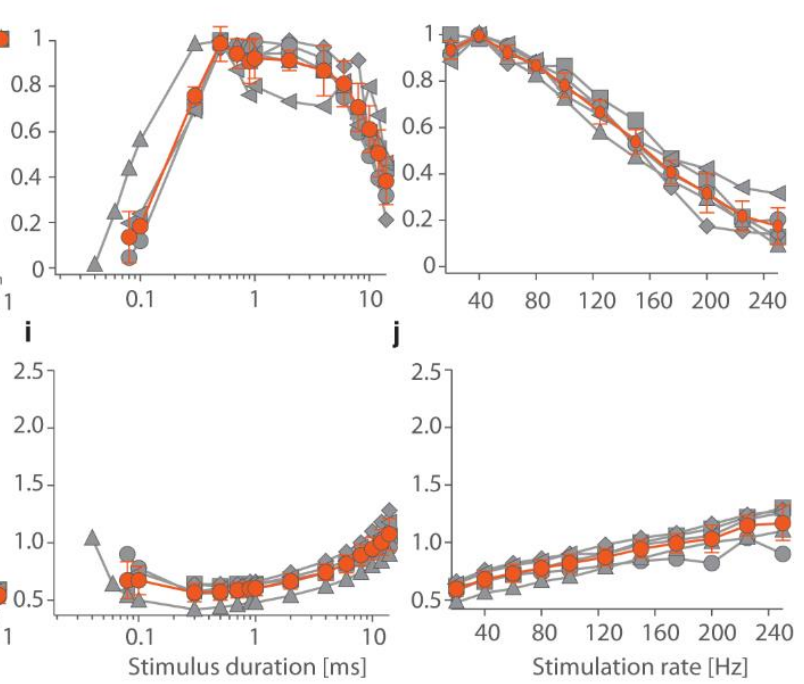

Supplementary Figure 10. f-Chrimson expression and function in SGNs in aged BL6/J mice. (a) Projections of confocal cryosections (scale bar: 50 $\mu \mathrm{m}$ ) with EYFP (green) and parvalbumin (magenta) immunofluorescence of SGNs in three cochlear regions. Insets (scale bar: 10 $\mu \mathrm{m}$ ) show close-up images of single z-sections of the same images. (b) Confocal z-sections $(0.25 \mu \mathrm{m}$ step) showing EYFP expression (green) in parvalbumin positive SGNs (magenta) from cochlear cryosections of the injected ear of the animal shown in a. Scale bar: $20 \mu \mathrm{m}$. (c) Fraction of EYFP-positive SGNs (identified by parvalbumin immunofluorescence, parvalbumin ${ }^{+}$) and (d) density of parvalbumin ${ }^{+}$SGNs (\#cells per $10^{4} \mu \mathrm{m}^{2}$ ) obtained from data as in Fig. 4.4F for apex, mid-cochlea (Mid) and base of the cochlea. Symbols mark results from individual animals (also for $\mathbf{e}-\mathbf{j}, n=5$ ) and box plots show $10^{\text {th }}, 25^{\text {th }}, 50^{\text {th }}, 75^{\text {th }}$ and $90^{\text {th }}$ percentiles of the injected (orange) and control (magenta) cochleae (one-way ANOVA, $p=0.1646 ; \mathrm{F}_{2,12}=2.1$; post-hoc Tukey's test for comparison of expression, $P>0.05$ for all pairwise comparisons; t-test for comparison of density, $\mathrm{L}_{\text {apex }}$ vs $\mathrm{R}_{\text {apex }}, \mathrm{L}_{\text {mid }}$ vs $\mathrm{R}_{\text {mid, }} \mathrm{L}_{\text {base }} \mathrm{vs} \mathrm{R}_{\text {base }} p>0.05$ for all comparisons). (e-g) Normalized P1-N1-amplitude as a function of laser intensity (e, $1 \mathrm{~ms}$ at $20 \mathrm{~Hz}$ ), pulse duration (f, $11 \mathrm{~mW}$ at $20 \mathrm{~Hz}$ ), and stimulus rate $(\mathrm{g}, 11 \mathrm{~mW}, 1 \mathrm{~ms}$ ). Group average (lines) and s.d. (error bars) are shown in orange (same for $\mathbf{g - i})$. (h-j) P1-latency as a function of laser intensity (h, as in $\mathbf{e}$ ), duration (i, as in $\mathbf{f}$ ), and rate ( $\mathbf{j}$, as in $\mathbf{g}$ ). 

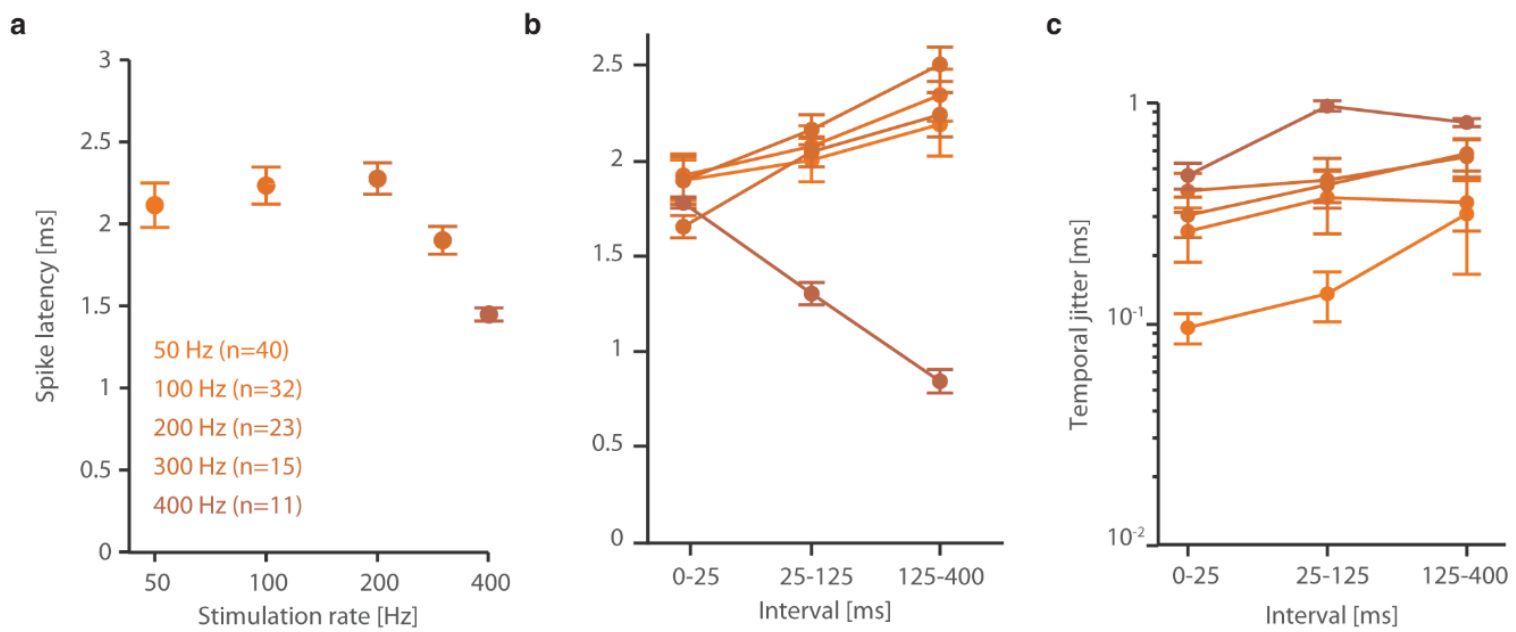

Supplementary Figure 11. Latency and temporal jitter of f-Chrimson-mediated spiking in SGNs. (a) Spike latency to light pulse onset as a function of stimulus rate (1 ms pulses of 11 $\mathrm{mW}$ over 400 or $900 \mathrm{~ms}$ stimulation, leaving $100 \mathrm{~ms}$ of inter-train recovery, only first $400 \mathrm{~ms}$ were analyzed here). Mean estimates were obtained for trials containing in average 5 spikes or more for each neuron. (b) Spike latency considering three time-windows from light pulse-train onset. (c) Same analysis as in (b) for temporal jitter of spikes, showing that this parameter tends to increase at later time windows along the train, which reflects a decrease in temporal precision of spiking. Data points show mean \pm s.e.m. (error bars). Number of units (from 5 mice) included for each stimulation frequency (color coded) is shown in $\mathbf{a}$. 
Supplementary Table 1: Relative calcium permeabilities of channelrhodopsin variants. Shown are the average, relative calcium permeabilities $\left(\mathrm{P}_{\mathrm{Ca}} / \mathrm{P}_{\mathrm{Na}}, \mathrm{n}=3-5\right)$ and the corresponding standard deviations. The values were determined in NG cells and HEK cells as described in the Methods section. ${ }^{\mathrm{a}}$ The relative calcium permeabilities were determined in HEK cells. ${ }^{\mathrm{b}}$ taken from (Kleinlogel et al., 2011). ${ }^{\mathrm{c}}$ The relative calcium permeabilities were determined in NG cells. ${ }^{\mathrm{d}}$ Determination of relative calcium permeability not possible due to strongly reduced calcium currents.

\begin{tabular}{|c|c|}
\hline Channelrhodopsin variant & $\mathbf{P}_{\mathrm{Ca}} / \mathbf{P}_{\mathrm{Na}}$ \\
\hline ChR2 & $0.13 \pm 0.01^{\mathrm{a}}$ \\
\hline ChR2 L132C & $0.24^{\mathrm{a}, \mathrm{b}}$ \\
\hline ChR2 F219Y & $0.30 \pm 0.02^{\mathrm{a}}$ \\
\hline ReaChR & $0.14 \pm 0.02^{\mathrm{c}}$ \\
\hline ReaChR L172C & $0.19 \pm 0.01^{\mathrm{c}}$ \\
\hline ReaChR F259Y & $0.22 \pm 0.02^{\mathrm{c}}$ \\
\hline Chrimson & $0.29 \pm 0.2^{\mathrm{c}}$ \\
\hline Chrimson L174C & $\mathbf{c , d}$ \\
\hline Chrimson Y261F & $\mathbf{c , d}$ \\
\hline
\end{tabular}




\section{Supplementary Table 2: Current densities of Chrimson and Chrimson mutants}

Current densities $\left(\mathrm{J}_{-} 60 \mathrm{mV}\right)$ of Chrimson and Chrimson mutants. Shown are the average current densities $(n=7-11)$ and the corresponding standard deviations. NG cells transiently expressing Chrimson-EYFP and Chrimson-EYFP mutants were investigated by patch-clamp measurements in the whole-cell configuration at a membrane potential of $-60 \mathrm{mV}$. The $\mathrm{J}_{-60 \mathrm{mV}}$ values were determined as described in the Methods section. \# Significantly different current density compared to Chrimson a) wt b) K176R c) S267M d) Y268F e) Y261F f) S267M/Y268F g) Y261F/S267M h) K176R/S267M/Y268F i) Y261F/S267M/Y268F j) K176R/Y261F/S267M k) $\mathrm{K} 176 \mathrm{R} / \mathrm{Y} 261 \mathrm{~F} / \mathrm{S} 267 \mathrm{M} / \mathrm{Y} 268 \mathrm{~F}$ (ANOVA with Bonferroni post-hoc test: $* p<0.05 * * p<0.01$ $* * * p<0.001)^{\S}$ variants with higher current density (no significantly different current densities within the group) ${ }^{\$}$ mutants with lower current density (no significantly different current densities within the group) ${ }^{\S}$ no significantly different current density compared to the high current density group and significantly different current density compared to all members of the low current density group, but Chrimson K176R and Chrimson S267M/Y268F

\begin{tabular}{|c|c|}
\hline Chrimson variant & $J_{-60 \mathrm{mv}}[\mathrm{pA} / \mathrm{pF}]$ \\
\hline wt $\S$ & $24.0 \pm 6.8 \quad \# b^{*}, d^{* * *}, f^{*}, h^{* * *}, i^{* * *}, j^{*}, k^{* * *}$ \\
\hline $\mathrm{K} 176 \mathrm{R}^{\$}$ & $10.1 \pm 6.9 \quad \# \mathrm{a}^{*}, \mathrm{e}^{\star \star *}, \mathrm{~g}^{\star \star *}$ \\
\hline S267M §§ & 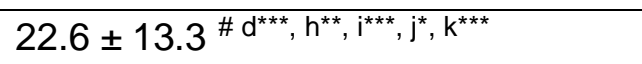 \\
\hline Y268F \$ & $3.5 \pm 1.6 \quad \# a^{* \star *}, \mathrm{c}^{\star \star \star *}, \mathrm{e}^{* \star \star}, \mathrm{g}^{* \star \star}$ \\
\hline Y261F § & $33.3 \pm 8.6 \quad \# b^{* \star *}, d^{* \star *}, f^{\star \star *}, h^{* \star *}, j^{* * *}, j^{* \star *}, k^{* \star *}$ \\
\hline S267M/Y268F \$ & $10.8 \pm 5.9 \quad \# \mathrm{a}^{*}, \mathrm{e}^{\star \star \star *}, \mathrm{~g}^{\star \star \star *}$ \\
\hline Y261F/S267M § & $34.2 \pm 12.7 \# b^{* * *}, d^{* \star *}, f^{* \star *}, h^{* * *}, i^{* * *}, j^{* \star *}, k^{* \star *}$ \\
\hline K176R/S267M/Y268F ${ }^{\$}$ & $\# \mathrm{a}^{* * *}, \mathrm{c}^{\star *}, \mathrm{e}^{* \star *}, \mathrm{~g}^{* * *}$ \\
\hline Y261F/S267M/Y268F \$ & $\# \mathrm{a}^{\star * \star}, \mathrm{c}^{\star * \star}, \mathrm{e}^{\star \star *}, \mathrm{~g}^{\star \star *}$ \\
\hline $\mathrm{K} 176 \mathrm{R} / \mathrm{Y} 261 \mathrm{~F} / \mathrm{S} 267 \mathrm{M}^{\$}$ & $8.3 \pm 5.3 \quad \# a^{*}, c^{*}, e^{* \star *}, g^{* \star *}$ \\
\hline K176R/Y261F/S267M/Y268F \$ & $\# a^{\star \star \star}, c^{\star \star \star}, e^{\star \star \star}, g^{\star \star \star}$ \\
\hline
\end{tabular}


Supplementary Table 3: Relative potassium and proton permeabilities of Chrimson variants.

Shown are the average, relative potassium permeabilities $\left(\mathrm{P}_{\mathrm{K}} / \mathrm{P}_{\mathrm{Na}}, \mathrm{n}=5\right)$ and the average, relative proton permeabilities $\left(\mathrm{P}_{\mathrm{H}} / \mathrm{P}_{\mathrm{Na}}, \mathrm{n}=7\right)$ of Chrimson-EYFP and Chrimson-EYFP Y261F/S267M. The values were determined in Xenopus laevis oocytes as described in the Methods section.

\begin{tabular}{|c|c|c|}
\hline Chrimson & $\mathbf{P}_{\mathrm{K}} / \mathbf{P}_{\mathrm{Na}}$ & $\mathbf{P}_{\mathrm{H}} / \mathbf{P}_{\mathrm{Na}}$ \\
\hline wt & $0.62 \pm 0.04$ & $\sim 10^{6}$ \\
\hline Y261F/S267M & $0.63 \pm 0.11$ & $\sim 10^{6}$ \\
\hline
\end{tabular}

Supplementary Table 4: Off kinetics ( $\left.\tau_{\text {off }}\right)$, current density (J-70mv) and the lowest light intensity required to induce action potentials with a probability of $100 \%\left(\mathrm{I}_{100}\right)$.

Shown are the average $\tau_{\text {off }}(n=3-11)$, the average $J_{-70 m V}(n=10-14)$, the average $I_{100}(n=15)$ and the corresponding standard deviations. The $\tau_{\text {off }}, J_{-70 \mathrm{mV}}$ and $\mathrm{I}_{100}$ values were determined in rat hippocampal neurons as described in the Methods section.

\begin{tabular}{|c|c|c|c|c|}
\hline Chrimson variant & $\tau_{\text {off }}[\mathrm{ms}]$ & $\mathrm{J}_{-70 \mathrm{mv}}[\mathrm{pA} / \mathrm{pF}]$ & $\mathrm{I}_{100}[\mathrm{~mW} \mathrm{~mm}-2]$ & $<\mathrm{l}_{100}>\left[\mathrm{mW} \mathrm{mm}^{-2}\right]$ \\
\hline wt & $35.1 \pm 9.4$ & $40.3 \pm 14.3$ & $0.09-0.70$ & $0.28 \pm 0.18$ \\
\hline Y261F/S267M & $4.7 \pm 1.8$ & $29.9 \pm 15.9$ & $0.37-1.27$ & $0.65 \pm 0.31$ \\
\hline K176R/ Y261F/ S267M & $3.8 \pm 0.4$ & $26.5 \pm 9.9$ & $0.09-3.18$ & $1.25 \pm 1.02$ \\
\hline
\end{tabular}




\section{Characterization of optogenetic responses to varying light intensity in f-Chrimson-expressing SGNs}

\subsection{Introduction}

In the previous chapter, I characterized both temporal precision and fidelity aspects of firing upon optogenetic stimulation of SGNs expressing f-Chrimson, with a focus on increasing pulse rates. As stimulation rate, light intensity is another key stimulus feature in shaping the response patterns of ChR-transduced neurons. However, in chapter 4, neural responses to changes in stimulus level were only assessed at the population level by means of oABRs.

As discussed in the general introduction, RLFs describe the relationship between spike rate and stimulus level. Hence, the dynamic range of individual fibers can be inferred from them. In response to acoustic stimulation, there seems to be an inverse correlation between both dynamic range and threshold on the one hand and SR on the other hand (Yates et al., 1990).

In response to sound, SGNs code stimulus intensity using spike rate, meaning that as stimulus levels increase, spike rates also grow over a certain range. Single SGNs are able to encode changes in sound intensity of about 20-30 dB SPL as measured in response to pure tones $(\sim 2$ orders of magnitude in terms of signal amplitude) (Sachs and Abbas, 1974). However, at the systems level, the full spectrum of sound intensities that an organism is able to encode and perceive as different is much larger, spanning 6 orders of magnitude in humans (Hudspeth, 2014; Viemeister and Bacon, 1988). This discrepancy is known as the "dynamic range problem" (Evans, 1981). To achieve this, the auditory system, at least to some degree, makes use of the individual, much narrower input-output functions of its constituent elements, so that they are staggered along the full range of discernible intensities. This is applicable not only to SGNs but also to neurons at higher centers, as the auditory cortex (Watkins and Barbour, 2011). Furthermore, in natural hearing, increments in sound level correlate with the number of recruited SGNs (Furman et al., 2013). Thus, the combination of individual functions with different thresholds (Evans, 1972; Liberman, 1978) allows to count on a pool of SGNs capable to modulate their firing rate at a given intensity, for which other SGNs show a saturated rate while a third group displays baseline firing levels (Sachs and Abbas, 1974). Despite the fact that the vast majority of SGNs show saturated responses at high sound levels (> $80 \mathrm{~dB}$ ) (Evans, 1972; Liberman, 1978), a minimal fraction of SGNs with unsaturated rates at high intensities 
innervating each frequency region (probably 1-6 fibers suffice) could account for the ability to encode these loud sounds (Viemeister, 1983, 1988). However, data from fibers with such high thresholds are lacking. Therefore, this suggests that additional mechanisms contribute to the broad range of intensities that can be perceived, e.g. the dynamic range adaptation to the overall loudness at a certain time (Barbour, 2011; Wen et al., 2009). In a nutshell, the auditory system deals with such large changes in stimulus intensities by dynamically adjusting its responses at two levels: regulating both the behavior of its individual elements and the overall responsiveness of the system as a whole.

How ChR-expressing SGNs respond to changes in light intensity has not yet been extensively studied. Most of the still few published articles have focused on describing single SGN responses to increasing stimulation rates, and characterizing light sensitivity at the population level (Hernandez et al., 2014a; Wrobel et al., 2018). Other studies have only briefly investigated this issue in neurons at higher auditory centers (Duarte et al., 2018; Hight et al., 2015). In order to mimic the physiological performance of SGNs, optogenetic hearing requires to operate not only at high rates of firing, but also with a range of stimulus intensities that allow to recruit groups of SGNs in a dynamic manner, as in natural hearing. This means that light sensitivity, i.e. ChR expression levels (Schoenenberger et al., 2008), as well as position relative to a given emitter should vary, so that the distribution of thresholds among SGNs at a given tonotopic region is heterogeneous (Jeschke and Moser, 2015). This would contribute to increase the dynamic range of the population by sequentially recruiting groups of SGNs as light intensities rise, and might be crucial to improve speech perception in noise with respect to current eCIs (Weiss et al., 2016; Zeng and Galvin, 1999). Therefore, characterizing optogenetic responses of SGNs at different intensities is of major importance prior to the clinical translation of oCIs. Moreover, studying SGNs responses to varying light intensities would allow to better compare optogenetic and electrical hearing and to help to answer open questions: does the dynamic range of optogenetic hearing outperform that of electrical hearing at the single fiber level $(\sim 1 \mathrm{~dB}$ (Miller et al., 2006))? How do the energies required to elicit neural responses in optogenetic and electrical hearing compare? How reliable are SGN responses at low light levels? This chapter aims at shedding light on these issues. 


\subsection{Materials and methods}

The AAV used here was the same that we used in Chapter 4 (AAV2/6-hSyn-f-Chrimson-EYFP). Round window injections (performed by Dr. Vladan Rankovic and Christiane Senger-Freitag), optical stimulation in vivo, immunostaining and imaging of cochlear cryosections, ABR recordings and juxtacellular single-unit recordings procedures were detailed in Chapter 4.

\section{Stimulation paradigm for construction of rate-level functions}

Once a positive oABR signal was measured and surgery was completed on 8-16 week-old C57BL6/J mice injected at P5-7 with AAV2/6-f-Chrimson-EYFP (or PBS in control experiments), a $50 \mu \mathrm{m}$ optical fiber was placed in the round window niche to deliver the light of a $594 \mathrm{~nm}$ laser (OBIS LS OPSL; $100 \mathrm{~mW}$, Coherent Inc.). Glass microelectrodes (World Precision Instruments) filled with $3 \mathrm{M} \mathrm{NaCl}$ were positioned on the recording area over the anteroventral cochlear nucleus. A search stimulus consisting on $400 \mathrm{~ms}-$ long pulse trains (100 ms inter-train recovery) at $\sim 14 \mathrm{~mW}, 100 \mathrm{~Hz}, 1 \mathrm{~ms}$ pulse width, was used to find light-responsive fibers. Microelectrodes were advanced stepwise (1.5 $\mu \mathrm{m}$ steps) through the neural tissue up to $\sim 2 \mathrm{~mm}$ depth using a micro-positioner (Luigs \& Neumann). Upon finding light-responsive fibers (at depths higher than $1 \mathrm{~mm}$ relative to the cochlear nucleus surface), responses to pulse trains similar to the search stimulus were recorded over 20 iterations per light intensity tested $(0-18.3 \mathrm{~mW})$ in no particular order. Spontaneous activity was recorded over $10 \mathrm{~s}$ without stimulation.

For acoustical stimulation experiments, 6-11 week-old C57BL6/J wild-type mice with normal hearing thresholds (30-40 dB SPL at click-evoked ABR measurements) were used. In these experiments, the middle ear surgery was spared and an Avisoft ScanSpeak Ultrasonic Speaker (Avisoft Bioacoustics) was used for stimulation instead of the optical fiber. The search stimulus here consisted of $400 \mathrm{~ms}$ click trains with $300 \mu$ s click width at $85 \mathrm{~dB}$ SPL (peak equivalent, pe) delivered at $100 \mathrm{~Hz}$, and the range of sound intensities used was 0-110 dB SPL (pe).

\section{Data analysis}

Spikes were detected and sorted; vector strength, spike probability, firing rate, first spike latency and jitter were extracted using a Matlab (MathWorks) custom-written algorithm. The spike probability was calculated by detecting whether or not spikes occurred in a time window equal to the stimulation cycle (giving a binary value of 0 or 1 per cycle) and averaging the results across trials. The extent of phase-locking was assessed with the vector strength (Goldberg and 
Brown, 1969), using a cycle starting with the onset of a light pulse and ending with the onset of

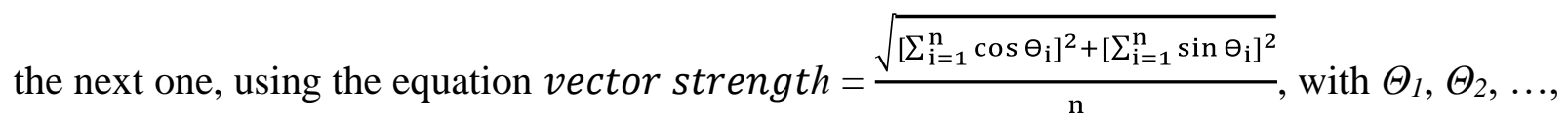
$\Theta_{n}$ marking cycle phases in which spikes occurred. Significance of vector strength was assessed by means of the Rayleigh test, so that the null hypothesis (a uniform distribution of events) was rejected if $L>13.8$, at $\alpha=0.001$ (Hillery and Narins, 1987) and insignificant vector strength were set to 0 . Threshold was determined as the stimulus intensity eliciting $10 \%$ of the difference between spontaneous and maximal driven rate (Sachs and Abbas, 1974). Rate-level functions were constructed by plotting the average firing rate per stimulus intensity tested. The monotonicity index was calculated as the ratio between the firing rate at maximum intensity and the maximal firing rate. Dynamic range was calculated for rate-level functions by fitting sigmoidal curves to the data, in order to calculate the stimulus intensities eliciting responses equal to $10 \%$ and $90 \%$ of the difference between spontaneous and maximal driven rate (Figure 5.1). First spike latency was estimated as the time from stimulus onset to spike peak. The temporal jitter of spikes was defined as the standard deviation of spike latency across trials using a time window equal to one stimulation cycle. The mean number of spikes per cycle was calculated as the average ratio between the number of spikes and the number of stimulation cycles across trials. The adaptation ratio was estimated as the ratio between the average firing rate during the first $100 \mathrm{~ms}$ of the stimulus train and the average firing rate over the full $400 \mathrm{~ms}$ length of the train.

Statistical tests were performed using Igor Pro 6.3 (Wavemetrics) and GraphPad Prism (GraphPad Software). Data was prepared for display using Adobe Illustrator (Adobe Systems). Averages in figures and references to data in the text were expressed as mean \pm s.e.m. Data was tested for normality (D’Agostino \& Pearson omnibus normality test or Shapiro-Wilk test) and homoscedasticity (F-test) prior to statistical comparison with the two-tailed unpaired Student's t-test, or the unpaired two-tailed Mann-Whitney U test. For evaluation of multiple groups, statistical significance was calculated by using the one-way Kruskal-Wallis test followed by Dunn's test for non-normally distributed data. Box-whisker plots in figures show 1st, 25th, 50th, 75th and 100th percentiles. Significant differences are indicated as $* p<0.05$, ** $p<0.01$, *** $p<0.001, * * * * p<0.0001$ were applicable. 
A

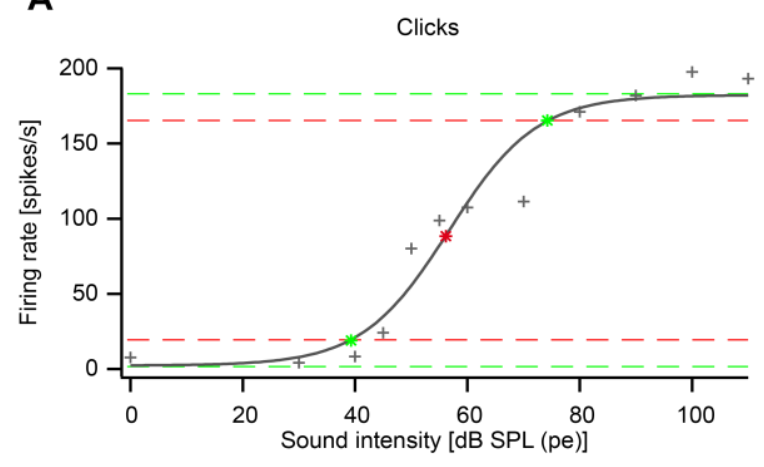

B

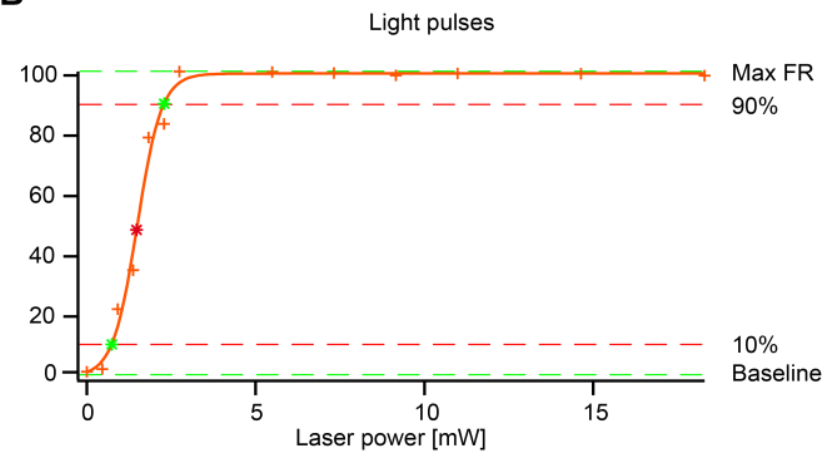

Figure 5.1: Calculation of the dynamic range of rate-level functions

Dynamic range was estimated by fitting sigmoidal functions and determining the baseline and maximal firing rate (Max FR) (green dashed lines) to calculate the $10 \%$ and $90 \%$ of the difference between the spontaneous and maximal driven rate (red dashed lines) and then subtract the stimulus intensity eliciting $10 \%$ of response to that eliciting $90 \%$ of response (green asterisks). Red asterisks mark the point where the function reaches $50 \%$ of the difference between spontaneous and maximal response. A: Fitting done for an exemplary rate-level function obtained from stimulation using acoustic clicks $(100 \mathrm{~Hz}, 300 \mu$ s click width; data points shown as crosses, as for B). B: Representation of a sigmoidal fit done for an exemplary ratelevel function obtained from optical stimulation (100 Hz, $1 \mathrm{~ms}$ pulse length).

\subsection{Results}

\subsubsection{Analysis of f-Chrimson-EYFP expression and opsin functionality}

In order to record optically-evoked juxtacellular single-unit activity from the auditory nerve, I first placed an optical fiber coupled to a $594 \mathrm{~nm}$ laser in the left side round window niche of 816 week-old C57BL6/J mice that were injected at P5-P7 with AAV2/6-hSyn-f-Chrimson-EYFP (as in Chapter 4). Upon light stimulation via the optical fiber, I recorded oABRs as a proxy of functional f-Chrimson expression in SGNs. I recorded a positive oABR signal in all tested animals $(5 / 5)$ but only $60 \%$ survived after craniotomy to render optically-evoked single-unit recordings (Figure 5.2A). PBS-injected mice of similar age did not show oABR responses (Figure 5.2B). Similarly, I used acoustic clicks to record acoustic ABRs (aABRs) and thus assess auditory function in mice used in acoustic experiments (Figure 5.2C).

Then, I analyzed f-Chrimson-EYFP expression by means of confocal imaging of cochlear cryosections and I found high and homogeneous EYFP expression levels ( 85\%) in SGNs throughout the cochlea ( $p>0.05, \mathrm{n}=5$; Dunn's test; Figure 5.3A,B). A minimal fraction of SGNs in the contralateral ear expressed the fusion protein (on average $\sim 5 \%$ ). Moreover, expression of f-Chrimson did not significantly affect SGN survival, as cell density was 


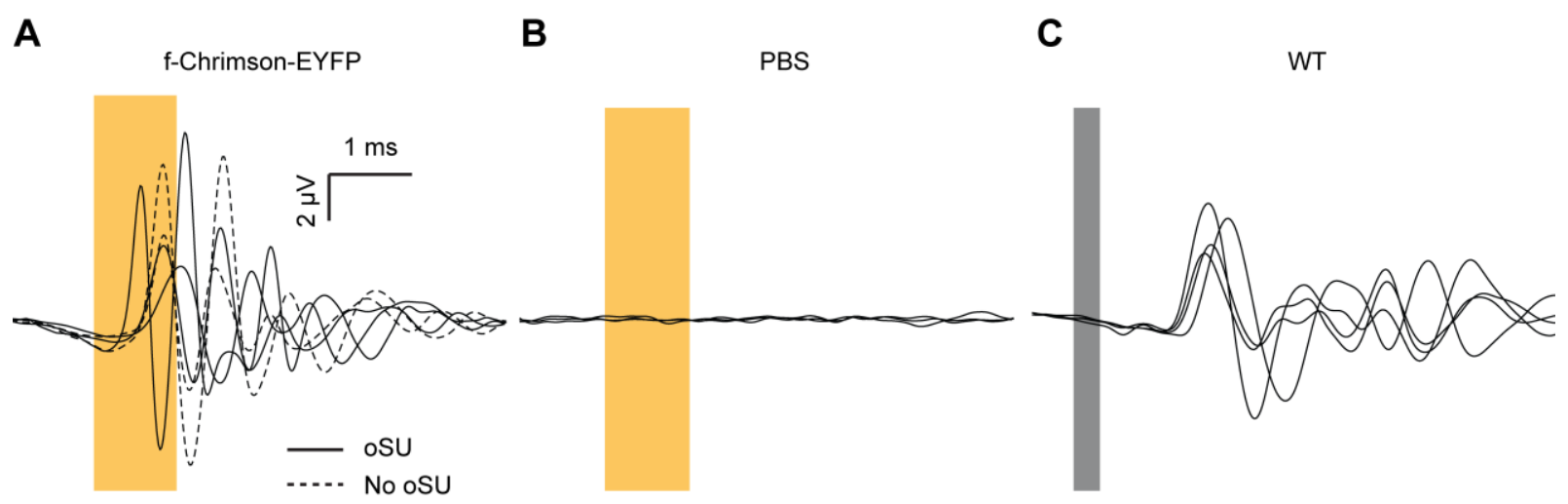

Figure 5.2: ABR recordings from $\mathrm{f}-\mathrm{Chrimson-transduced,} \mathrm{PBS}$-injected and WT mice A: All mice injected with AAV2/6-hSyn-f-Chrimson-EYFP (5/5) used in this chapter rendered a positive oABR signal $(18.3 \mathrm{~mW}, 1 \mathrm{~ms}$ pulse width at $20 \mathrm{~Hz})$. However, only $60 \%$ of them rendered single-unit recordings (solid lines). Scale bars apply to B and C. B: Contrarily, no oABR could be evoked in PBS-injected controls of similar age $(n=3)$. C: aABRs evoked in wild-type non-injected mice using acoustic clicks (0.3 ms click width, $80 \mathrm{~dB}$ SPL (pe) at $20 \mathrm{~Hz}$, $\mathrm{n}=4)$.

comparable in both ears, and also similar to PBS controls $(p>0.05, \mathrm{n}=5$; Dunn's test; Figure 5.3A,C).

\subsubsection{Optogenetic spike fidelity decays with stimulation rate}

In order to evaluate the effect of varying light intensity in f-Chrimson-expressing SGNs, stimulus parameters as pulse length and stimulation rate needed to be fixed in advance. Regarding pulse length, we used a standard of $1 \mathrm{~ms}$ in experiments conducted prior to these because oABR responses were optimal in response to this pulse duration (see Chapter 4). To select a stimulation rate that SGNs were able to follow reliably, I first investigated the relation between spike fidelity and stimulation frequency across rates ranging from 20 to $300 \mathrm{~Hz}$. As shown in Chapter 4, the majority of SGNs expressing f-Chrimson-EYFP show a cut-off frequency ranging from 200-300 Hz. For comparison, I adapted the stimulation paradigm applied in optogenetic stimulation experiments to present trains of acoustic square clicks to wild-type mice (400 ms-long click trains with $100 \mathrm{~ms}$ recovery over 20 iterations). In order to quantify spike fidelity, I calculated the mean probability of each SGN to fire a spike in response to each light pulse or click across stimulation rates. Results show that SGNs respond with a higher spike fidelity to click trains than to light pulse trains at high stimulation rates (Figure 5.4). 

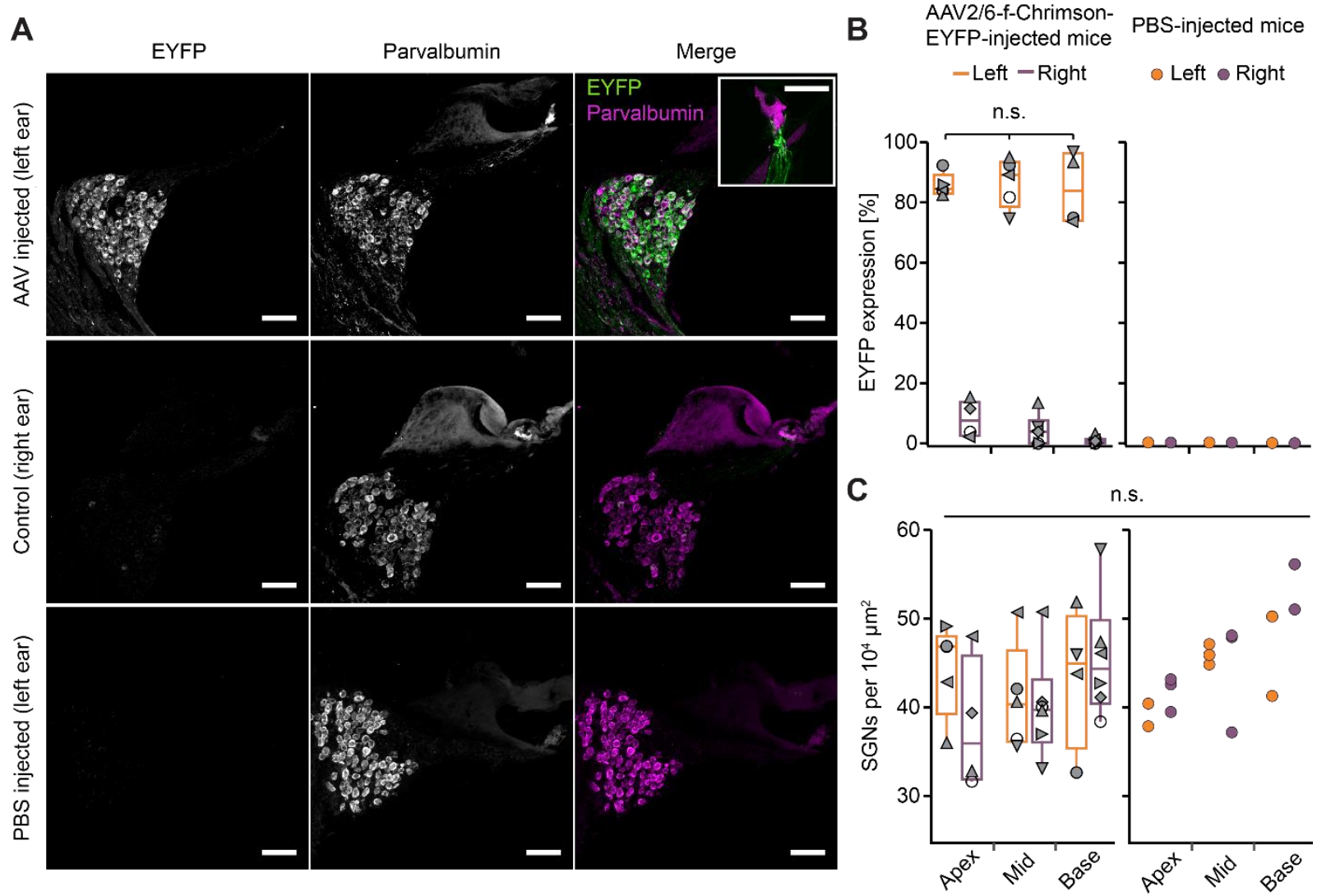

Figure 5.3: Expression of f-Chrimson-EYFP in SGNs

A: Confocal images from representative cochlear middle turn cryosections showing parvalbumin (magenta) and EYFP (green) immunofluorescence to mark f-Chrimson expression and SGNs, respectively. Scale bars: $50 \mu \mathrm{m}$. Inset (top right): parvalbumin-positive, EYFPnegative IHC with EYFP-positive SGN neurites. Scale bar: $20 \mu \mathrm{m}$. B: Boxplot showing the fraction of EYFP-expressing SGNs in the injected (left) and non-injected ear (right) $(n=7)$. Expression levels were homogeneous in the left side ( $p>0.05$; Dunn's test). No expression was found in PBS-injected animals $(n=3)$. C: Boxplot showing density of SGNs across cochlear turns, that was unaltered in mice injected with AAV2/6 $(n=7)$ or PBS $(n=3)(p>0.05$ for all comparisons; Mann-Whitney-U test)

Optogenetic stimulation triggers spikes almost after every light pulse at frequencies $20-100 \mathrm{~Hz}$, yielding a higher spike fidelity than that of acoustical stimulation at $50 \mathrm{~Hz}(p=0.0027$; MannWhitney-U test). In contrast, spike fidelity sharply decays at higher rates in response to optostimulation, whereas it is steadier in response to clicks (at 200 and $300 \mathrm{~Hz}, p<0.0001$; Mann-Whitney-U test). Responses to both clicks and light pulses were comparable at $100 \mathrm{~Hz}$ stimulation and therefore, I chose this rate as a standard to evaluate SGN responses to changing stimulus intensity. 


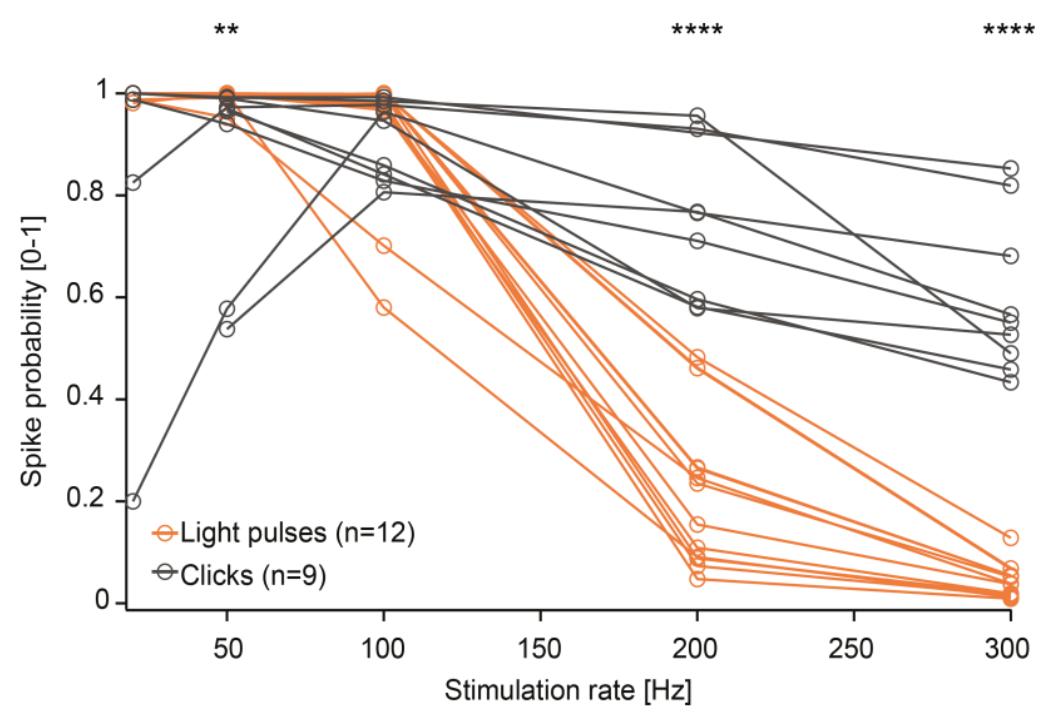

Figure 5.4: Spike fidelity decreases with increasing stimulation rates in optogenetic and acoustic stimulation experiments

Probability of SGN firing across different stimulation rates per acoustic click (grey, $100 \mathrm{~dB}$ SPL (pe), $300 \mu$ s click width at $100 \mathrm{~Hz} ; \mathrm{n}=12,3$ mice) and light pulse (orange, $18.3 \mathrm{~mW}, 1 \mathrm{~ms}$ pulse length at $100 \mathrm{~Hz} ; \mathrm{n}=9 ; 3$ mice) presented in a train over $400 \mathrm{~ms}$ leaving a recovery inter-train interval of $100 \mathrm{~ms}$ across repetitions per tested rate. Asterisks mark significant differences between stimulation modalities.

\subsubsection{Dynamic range of optogenetic stimulation}

In order to characterize SGN responses to varying light power, I first constructed RLFs by representing firing rates of light-stimulated SGNs in response to each intensity tested, and compared the results to stimulation using acoustic clicks (Figure 5.5).

In response to acoustic clicks presented at $100 \mathrm{~Hz}$, most RLFs appeared to grow with sound intensity with sloping saturation, i.e. firing rates were higher as sound intensity increased and rate did not saturate completely (Sachs and Abbas, 1974) (Figure 5.5A,C). In contrast, most light-stimulated SGNs reached flat saturation (Sachs and Abbas, 1974) at 100 spikes/s in response to light pulse trains delivered at $100 \mathrm{~Hz}$, revealing that most recorded SGNs fire one spike per pulse at saturating light intensities (27 out of 33 ANFs, $81.82 \%$, Figure 5.5B,D) while the remaining 6 responded with an average of $1.61 \pm 0.19$ spikes/pulse. In both stimulation modalities, firing rates increased with stimulus intensity. For characterization of RLFs, I compared responses to the entire $400 \mathrm{~ms}-$ long pulse train and to the first $100 \mathrm{~ms}$ (first 10 stimuli). There were no statistically significant differences in the responses using either time window (Figure 5.6). RLF growth was strongly monotonic for both stimulation modalities, but higher in acoustic RLFs for both time windows (monotonicity index, median $(400 \mathrm{~ms})=1.00$ 
vs $0.996 ; p=0.0142 ;$ median $(100 \mathrm{~ms})=1.00$ vs $0.93 ; p<0.0001$ in acoustical vs optical stimulation, respectively; Mann-Whitney-U test Figure 5.6A). RLFs at optogenetic stimulation grew with a steeper slope than at acoustical stimulation, as estimated by the maximal steepness of the RLFs (median $(400 \mathrm{~ms})=35.22 \mathrm{~Hz} / \mathrm{dB}(\mathrm{mW})$ vs $5.49 \mathrm{~Hz} / \mathrm{dB}$ SPL (pe), respectively; $p<$ 0.0001 ; median $(100 \mathrm{~ms})=34.36 \mathrm{~Hz} / \mathrm{dB}(\mathrm{mW})$ vs $5.87 \mathrm{~Hz} / \mathrm{dB}$ SPL (pe); $p<0.0001$; MannWhitney-U test; Figure 5.6B). Next, I estimated the dynamic range by fitting sigmoidal functions to RLFs. The dynamic range was defined as the range of stimulus levels for which firing rate was equal to $10 \%$ and $90 \%$ of the difference between spontaneous and maximal driven rate. Correspondingly, the steeper slope in response to optical stimulation is reflected in a narrower dynamic range of RLFs as compared to acoustical clicks $(3.87 \pm 0.47 \mathrm{~dB}(\mathrm{~mW})$ vs $31.29 \pm 4.46 \mathrm{~dB}$ SPL (pe), respectively; $p<0.0001$, t-test; Figure 5.6C) when considering the full length of the pulse train. Dynamic range was not significantly different when changing the analysis window from 400 to $100 \mathrm{~ms}$, and the differences observed between stimulus modalities remained significant $(2.74 \pm 0.33 \mathrm{~dB}(\mathrm{~mW})$ vs $19.77 \pm 3.89 \mathrm{~dB}$ SPL (pe) for optical vs acoustical, respectively; $p<0.0001$, t-test; Figure 5.6C).

I further investigated the relation between SR and threshold (defined as the stimulus intensity required to elicit a response above SR equal to $10 \%$ of the difference between spontaneous and maximal driven rate) (Figure 5.7). Neither acoustically-stimulated nor optically-stimulated SGNs showed a significant correlation between SR and click or light-pulse threshold, respectively $\left(r_{s}=0.06813 ; p=0.8201\right.$ for acoustical stimulation experiments, $r_{s}=-0.2610 ; p=$ 0.2122 for optogenetic stimulation experiments; Spearman correlation; Figure 5.7A,B). Compared to acoustically-stimulated SGNs, in experiments involving optogenetic stimulation only a subset of fibers showed spontaneous activity > 1 spike/s (29.41\% vs 64,28\% in optogenetic and acoustic stimulation experiments, respectively). Optically-stimulated SGNs showed an average threshold of $5.38 \pm 1.13 \mathrm{~dB}(\mathrm{~mW})$ relative to $0.5 \mathrm{~mW}$ while acousticallystimulated SGNs had a mean threshold of 58.56 $\pm 3.58 \mathrm{~dB}$ SPL (pe). The mean threshold of the three most sensitive SGNs found in optical experiments was $-1.25 \pm 0.64 \mathrm{~dB}(\mathrm{~mW})$ relative to $0.5 \mathrm{~mW}$ per pulse, probably reflecting optimal f-Chrimson expression and illumination conditions. I then calculated the stimulus intensity required for eliciting $50 \%$ of the difference between the spontaneous and maximal firing rate. For optical stimulation experiments, this estimate was on average $7.77 \pm 0.97 \mathrm{~dB}(\mathrm{~mW})$ relative to $0.5 \mathrm{~mW}$, while for acoustical stimulation experiments it reached $75.91 \pm 2.90 \mathrm{~dB}$ SPL (pe) (Figure 5.7C). To estimate the 
A

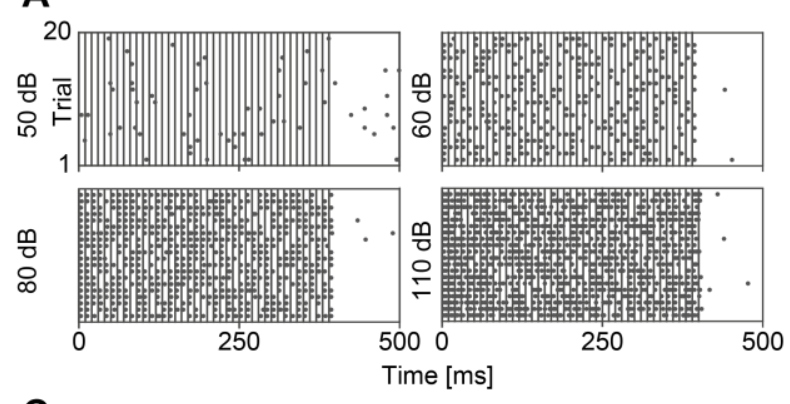

C

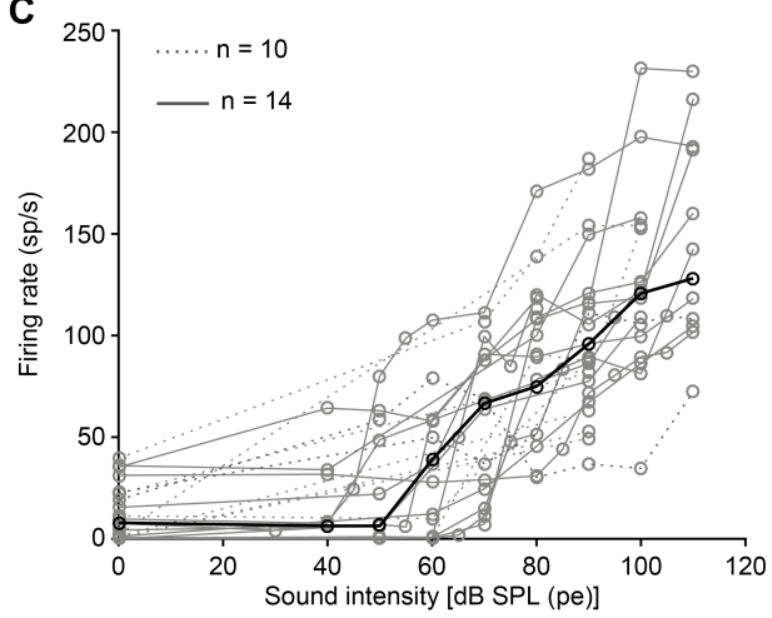

B

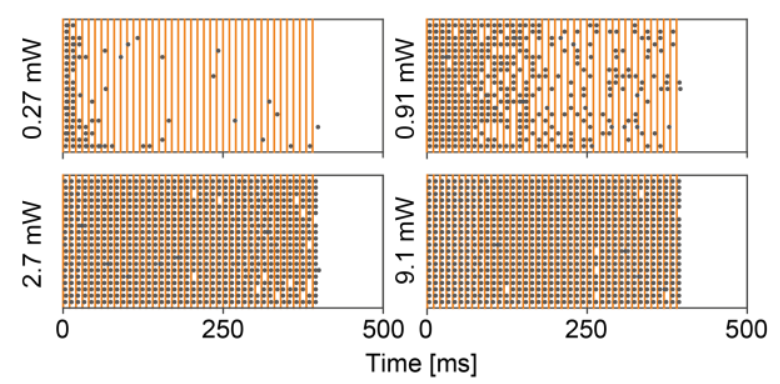

D

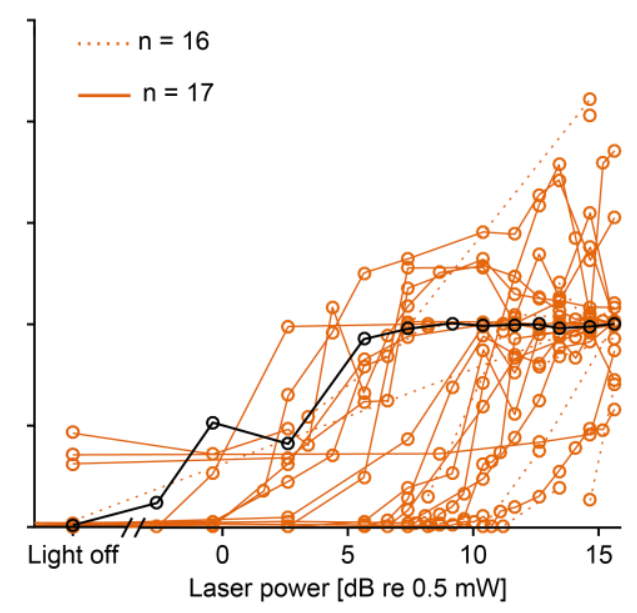

Figure 5.5: Rate-level functions in response to clicks and light pulses

A: Activity of an exemplary SGN in response to $300 \mu$ s clicks at $100 \mathrm{~Hz}$ at different sound intensities and B: at varying $594 \mathrm{~nm}$ light power in response to $1 \mathrm{~ms}$ pulses at $100 \mathrm{~Hz}$. C: RLFs in response to acoustic click trains for 24 single units from 4 mice. D: RLFs in response to light pulses displayed for 33 single units from 3 mice. Dashed lines in A and B show RLFs discarded for further analysis (clicks: $\mathrm{n}=10$ units from 4 mice; light-pulses: $\mathrm{n}=16$ units from 3 mice; also applies to C, D and E). Solid lines represent RLFs that were further analyzed in the next figure (clicks: $\mathrm{n}=14$ units from 4 mice; light-pulses: $\mathrm{n}=17$ units from 3 mice). Black traces in $\mathrm{C}$ and $\mathrm{D}$ show data for units in A and B respectively.

operating range of the population, I selected the three most responsive and the three least responsive units and calculated the difference in laser power between them at $50 \%$ of activation. Therefore, the operating range of optically-stimulated fibers was estimated in $8.33 \pm 0.16 \mathrm{~dB}$ $(\mathrm{mW})$.

\subsubsection{Temporal aspects of spiking in response to varying stimulus intensities}

In response to acoustical stimulation with clicks or optical stimulation with short light pulses, the latency to the first elicited spike relative to the click or pulse onset tended to decline with increasing stimulus intensity. At maximum stimulus levels, the mean first spike latency was 

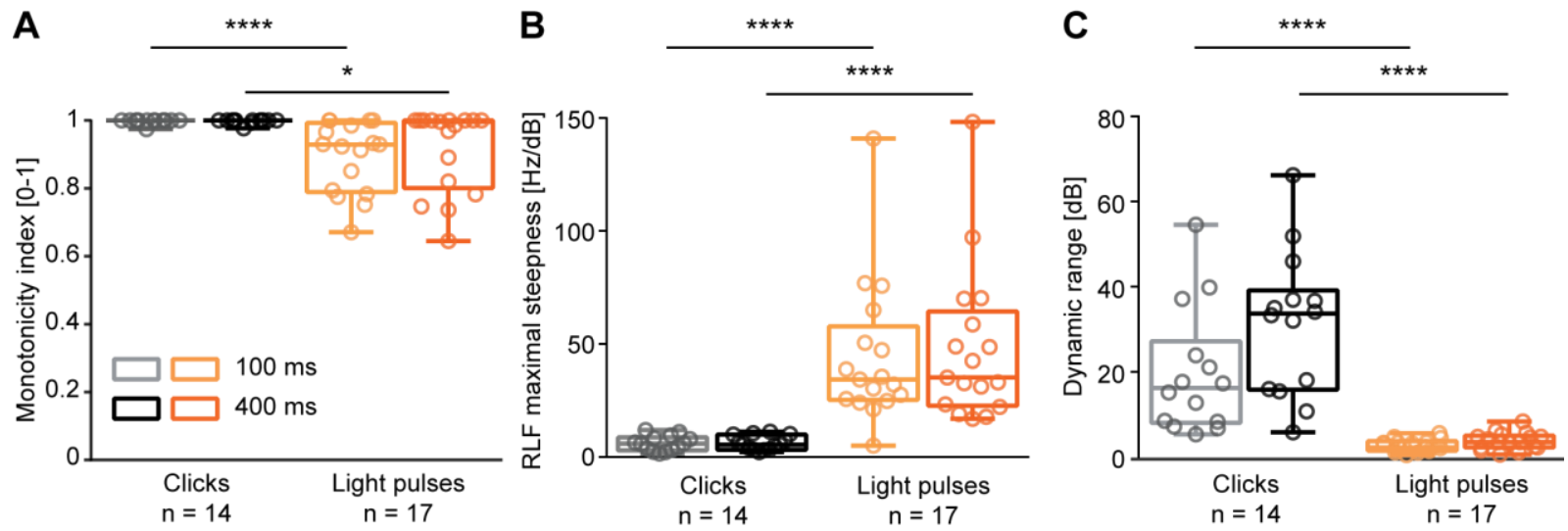

Figure 5.6: RLFs grow monotonically, steeper at optogenetic stimulation, leading to a reduced dynamic range

A: Monotonicity index of the RLFs (firing rate at maximal intensity over maximal firing rate) in response to clicks and light pulses. Color code applies to all panels. B: Maximal steepness of the RLFs in response to clicks and light pulses. C: Dynamic range of the RLFs (range of stimulus intensity levels eliciting 10 and $90 \%$ of the difference between spontaneous and maximal driven rate) in acoustical (dB SPL (pe)) and optogenetic stimulation $(\mathrm{dB}(\mathrm{mW})$.

A

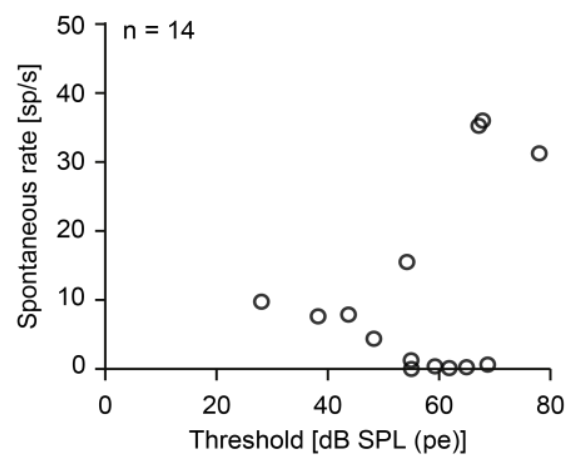

B

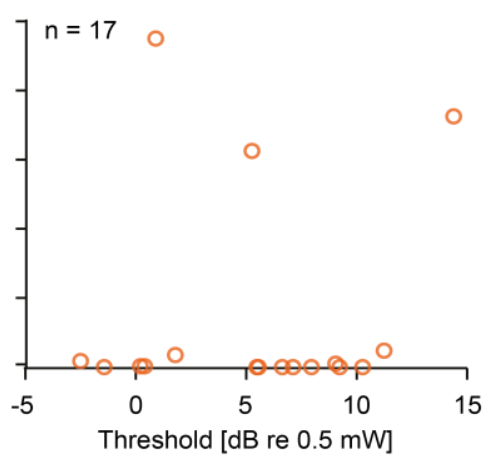

C

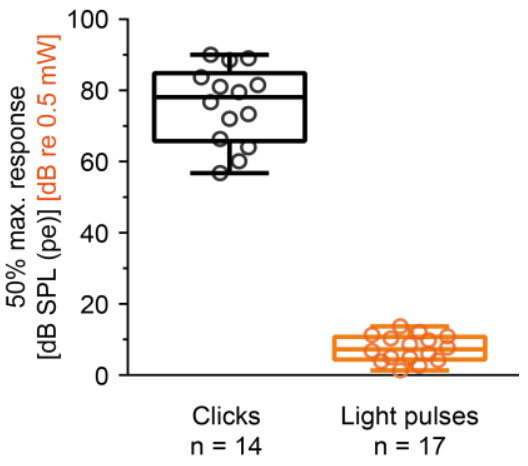

Figure 5.7: Spontaneous rate and response threshold in acoustically- and opticallystimulated ANFs

A: Scatter plot of threshold (stimulus intensity level for which the firing rate is equal to $10 \%$ of the difference between spontaneous and maximal driven rate) versus spontaneous rate for fibers stimulated with acoustic clicks $(n=14$ from 4 mice). B: Same plot as A representing responses from optically-stimulated fibers ( $\mathrm{n}=17$ from 3 mice). $\mathbf{C}$ : Box-whisker plots showing stimulus intensity levels eliciting $50 \%$ of the difference between spontaneous and maximal response for click stimulation (grey) and light pulse trains (orange).

significantly higher in response to clicks $(2.90 \pm 0.22 \mathrm{~ms})$ as compared to light-stimulated SGNs $(2.59 \pm 0.08 \mathrm{~ms})(p=0.0047$; Mann-Whitney-U test) (Figure 5.8A,B). Next, I quantified the temporal jitter of elicited spikes (defined as the standard deviation of first spike latency across trials) as an estimate, together with vector strength, of temporal precision of spiking. At 
maximum stimulus intensities, spiking was less precise in response to acoustical stimulation than in response to optical stimulation, as assessed by spike jitter of $1.06 \pm 0.17 \mathrm{~ms}$ for acoustic clicks and $0.39 \pm 0.05 \mathrm{~ms}$ for light pulses ( $p=0.0002$; Mann-Whitney-U test) (Figure 5.8C,D).

I further assessed the temporal precision of spiking by means of vector strength to measure the extent of phase locking across stimulus trains.

Vector strength rose steeply in response to both stimulation modalities after a certain stimulus level specific for each fiber was reached, giving rise to a heterogeneous population response. In response to acoustic clicks, once maximum phase locking level was reached, vector strength tended to decay at the highest intensities, while it was steadier once saturated in response to light pulses (medians: 0.84 for clicks, 0.98 for light pulses, $p<0.0001$; Mann-Whitney-U test, Figure 5.9).

\subsubsection{Optogenetic responses to low light intensities are more reliable at the beginning of the stimulation train}

To further characterize SGN responses at increasing light intensities, I calculated the mean number of spikes per pulse across different stimulus intensities. At maximum laser power, SGNs tended to fire $0.98 \pm 0.06$ spikes per pulse, which seems to be a saturation point in this case. The apparent higher number of spikes generated in response to acoustic click stimulation, amounting to $1.26 \pm 0.14$ spikes per click at maximum sound intensity levels, however, does not reach statistical significance ( $p=0.0974$; Mann-Whitney-U test). Further, the average number of spikes per click seems not to saturate at this point (Figure 5.10A,B). Next, to assess the reliability of spiking along the stimulus train, I defined the adaptation ratio as the ratio between the firing rate during the first $100 \mathrm{~ms}$ and the firing rate during the entire length of the train $(400 \mathrm{~ms})$. Results show that the adaptation ratio is maintained steady ( 1) across stimulation levels for acoustical stimulation (Figure 5.10C). Interestingly, at low laser intensities, the adaptation ratio is higher for optogenetic stimulation, while for higher intensities it approaches $~ 1$ (Figure 5.10D). This means that from threshold up to saturating levels, spikes at the beginning of the stimulus train are favored over a random distribution of spikes. At sound intensity levels of 40$50 \mathrm{~dB}$ SPL (pe), acoustically-driven SGNs show an adaptation ratio of $1.08 \pm 0.32$, significantly 
A

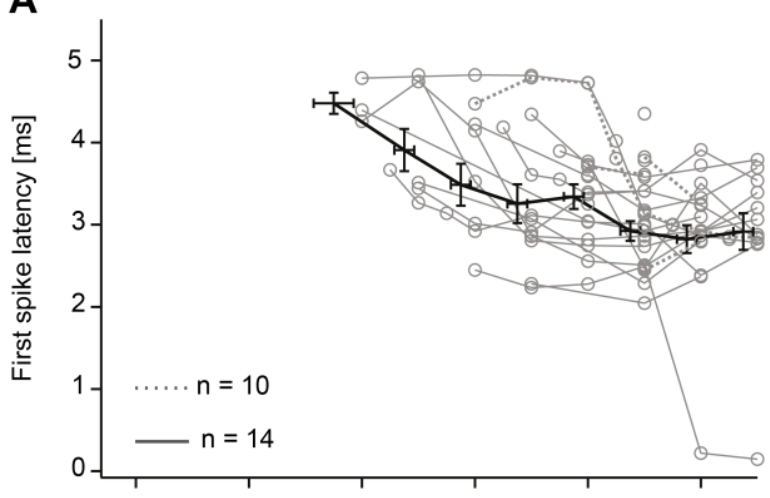

\section{C}

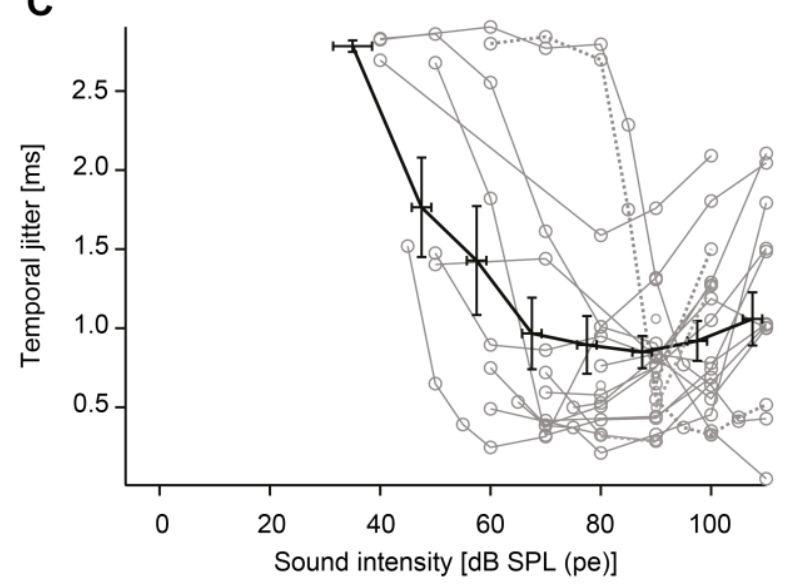

B

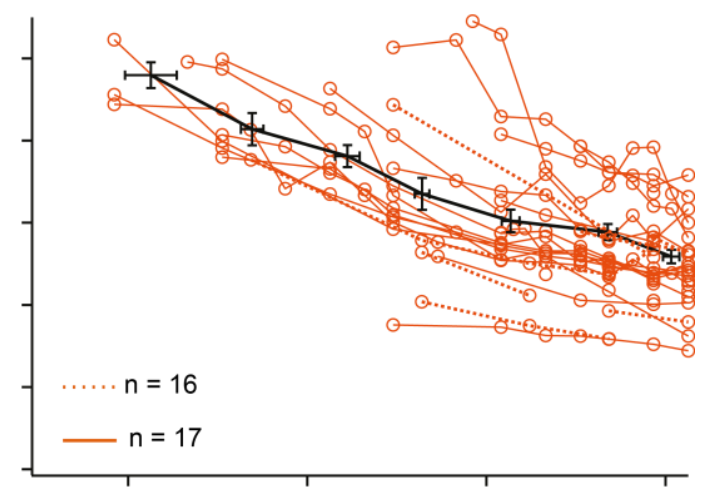

D

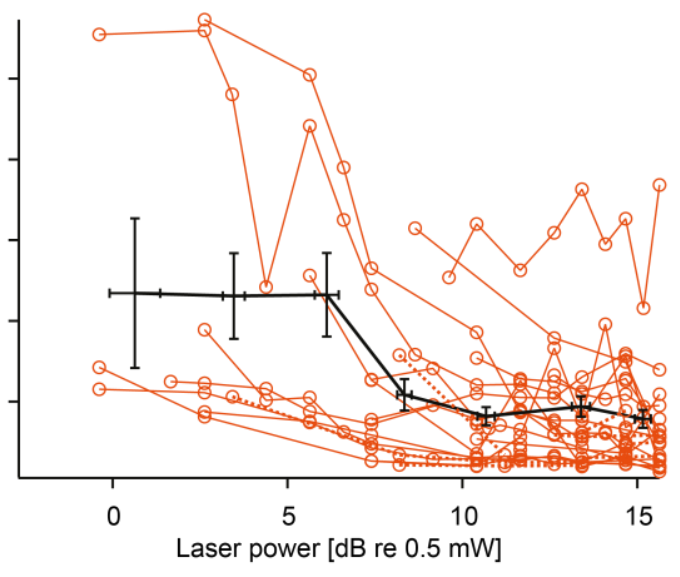

Figure 5.8: First spike latency and temporal jitter decrease with increasing click and light pulse intensity

A: Latency to the first spike per stimulation cycle elicited by clicks of increasing intensity $(\mathrm{n}=$ 24 from 4 mice). B: Same as A for optically stimulated fibers $(n=33$ from 3 mice). Dashed lines in A and B show fibers discarded for RLF analysis due to insufficient data points (clicks: $\mathrm{n}=10$ units from 4 mice; light-pulses: $\mathrm{n}=16$ units from 3 mice; also applies to $\mathrm{C}$ and D). Solid lines represent responses from fibers for which RLF was further analyzed in Figure 5.6 (clicks: $\mathrm{n}=14$ units from 4 mice; light-pulses: $\mathrm{n}=17$ units from 3 mice; also applies to $\mathrm{C}$ and $\mathrm{D}$ ). $\mathbf{C}$ : Jitter of the first elicited spike per stimulation cycle in acoustic experiments D: Same as C for optical stimulation experiments. Means ( \pm s.e.m.) are shown as black lines.

lower than that of $1.65 \pm 0.17$ for optically-stimulated SGNs at intensities of 2-4.5 $\mathrm{dB}(\mathrm{mW})$ relative to $0.5 \mathrm{~mW}$ ( $p=0.0149$; Mann-Whitney-U test).

\subsubsection{SGN responses to optogenetic stimulation of varying pulse rate and intensity}

During optogenetic stimulation recordings, I could hold a subset of 6 fibers after completing the previous recordings for a time long enough to preliminarily characterize SGN responses to varying stimulus rate and intensity together. Figure 5.11 shows RLFs of this subset of fibers at 

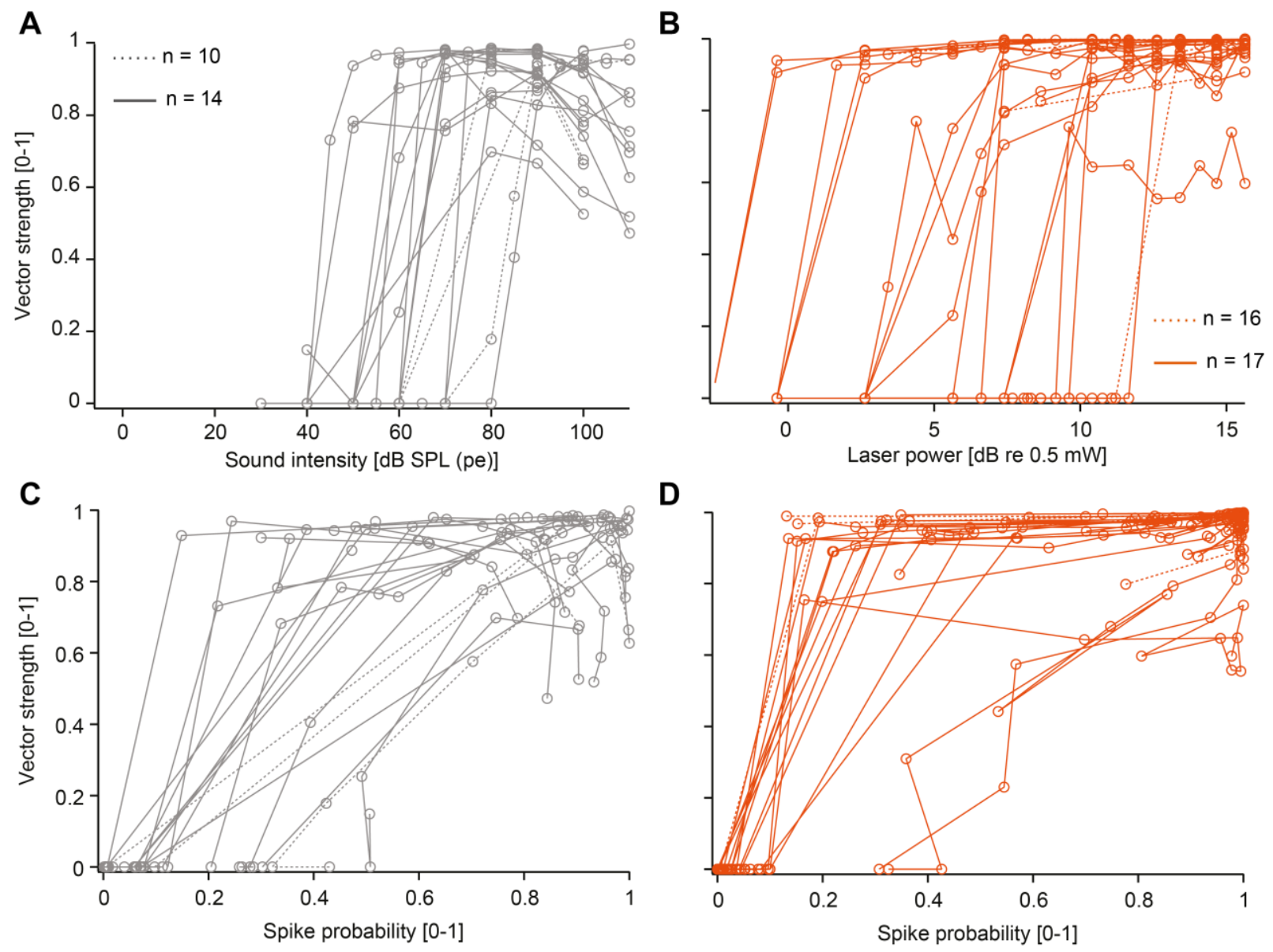

Figure 5.9: Phase locking increases with increasing stimulus intensity

A: Vector strength of the SGNs response to acoustic click trains of varying intensity ( $n=24$ from 4 mice). B: Same as A in response to light pulse trains $(n=33)$. Dashed lines in A and B show fibers discarded for RLF analysis due to insufficient data points (clicks: $\mathrm{n}=10$ units from 4 mice; light-pulses: $\mathrm{n}=16$ units from 3 mice; also applies to $\mathrm{C}$ and $\mathrm{D}$ ). Solid lines represent responses from fibers for which RLF was further analyzed in Figure 5.5 (clicks: $\mathrm{n}=14$ units from 4 mice; light-pulses: $\mathrm{n}=17$ units from 3 mice; also applies to $\mathrm{C}$ and $\mathrm{D}$ ). C: Variation of vector strength with spike probability in acoustically-stimulated fibers. D: Same as C for optically-stimulated fibers. Lines in C and D connect data points obtained from the same SGNs.

a pulse rate of $100 \mathrm{~Hz}$, and, superimposed, RLFs of these same SGNs at stimulation rates of 50 and $20 \mathrm{~Hz}$ (Figure 5.11A). When decreasing stimulation rate, SGN firing becomes more reliable for the same intensity levels (Figure 5.11B) ( $p=0.0062$; Dunn's test 20 vs $100 \mathrm{~Hz}$; n.s. for the rest of comparisons). Further examination led me to investigate whether threshold was reduced for these SGNs due to stimulus rate decline. Results in Figure 5.11C show that threshold levels were not significantly different at increasing stimulation rates of 20 to $100 \mathrm{~Hz}(p=0.1644$; Kruskal-Wallis test). 
A

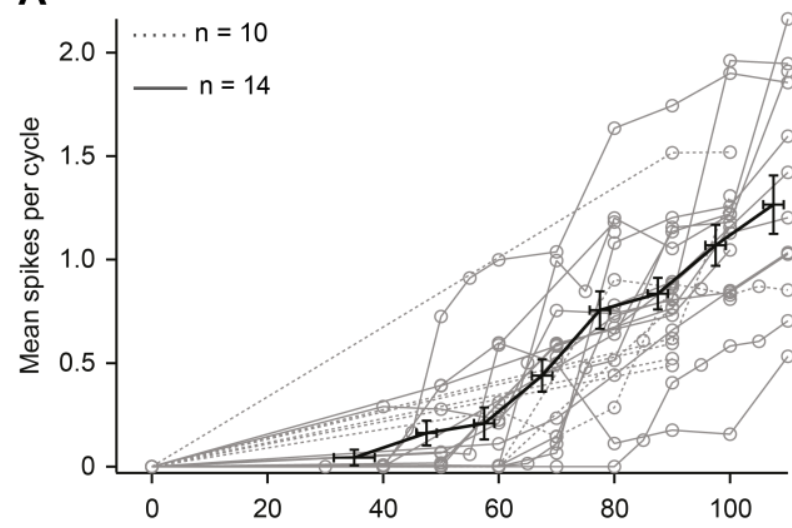

C

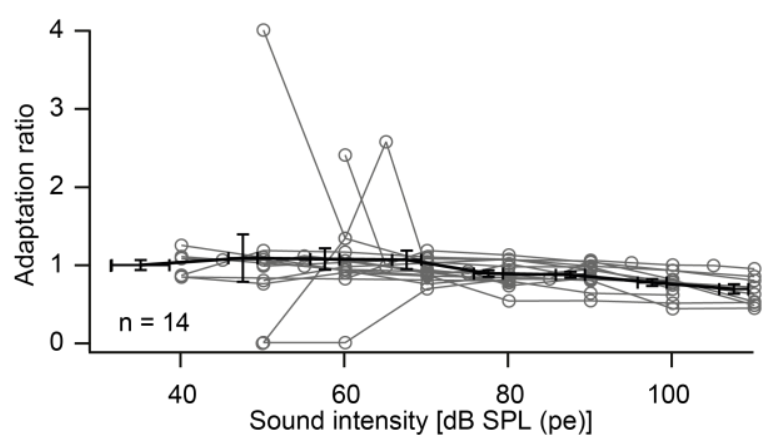

B

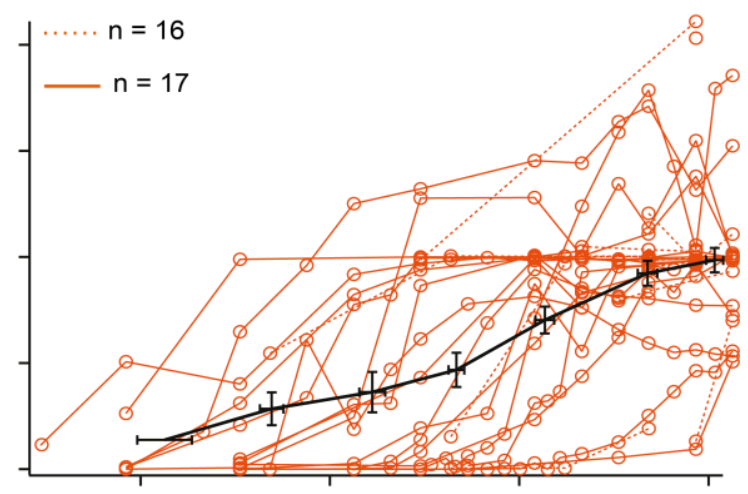

D

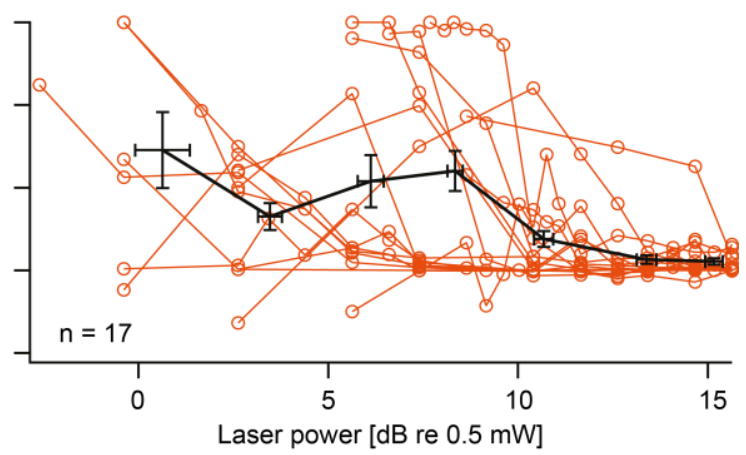

Figure 5.10: High light intensities trigger mainly one spike per pulse while low light intensities mainly elicit spikes at the beginning of the stimulation train

A: Mean number of spikes elicited per stimulation cycle in response to click stimulation at increasing intensities $(\mathrm{n}=24$ from 4 mice). B: Same as A in response to optogenetic stimulation ( $\mathrm{n}=33$ from 3 mice). Dashed lines in A and B show fibers discarded for RLF analysis due to insufficient data points (clicks: $\mathrm{n}=10$ units from 4 mice; light-pulses: $\mathrm{n}=16$ units from 3 mice). Solid lines represent responses from fibers for which RLF was further analyzed in Figure 5.5 (clicks: $\mathrm{n}=14$ units from 4 mice; light-pulses: $\mathrm{n}=17$ units from 3 mice). C: Adaptation ratio (mean firing rate during the first $100 \mathrm{~ms}$ over mean firing rate along the entire stimulation train) of SGN responses to acoustic clicks at increasing stimulus intensities $(n=14)$. D: Same as C for fibers stimulated with light pulse trains at increasing intensities $(n=17)$. Means $( \pm$ s.e.m. $)$ are shown as black lines.

\subsection{Discussion}

The data presented above shows how different measures of SGN spiking vary with stimulus intensity in response to acoustic clicks and optical pulses. As expected, firing rates increased in a level-dependent manner in both click and light pulse stimulation (Guo et al., 2015; Hight et al., 2015; Lin and Guinan Jr, 2000), similar to electrical stimulation (Miller et al., 1999, 2006). 

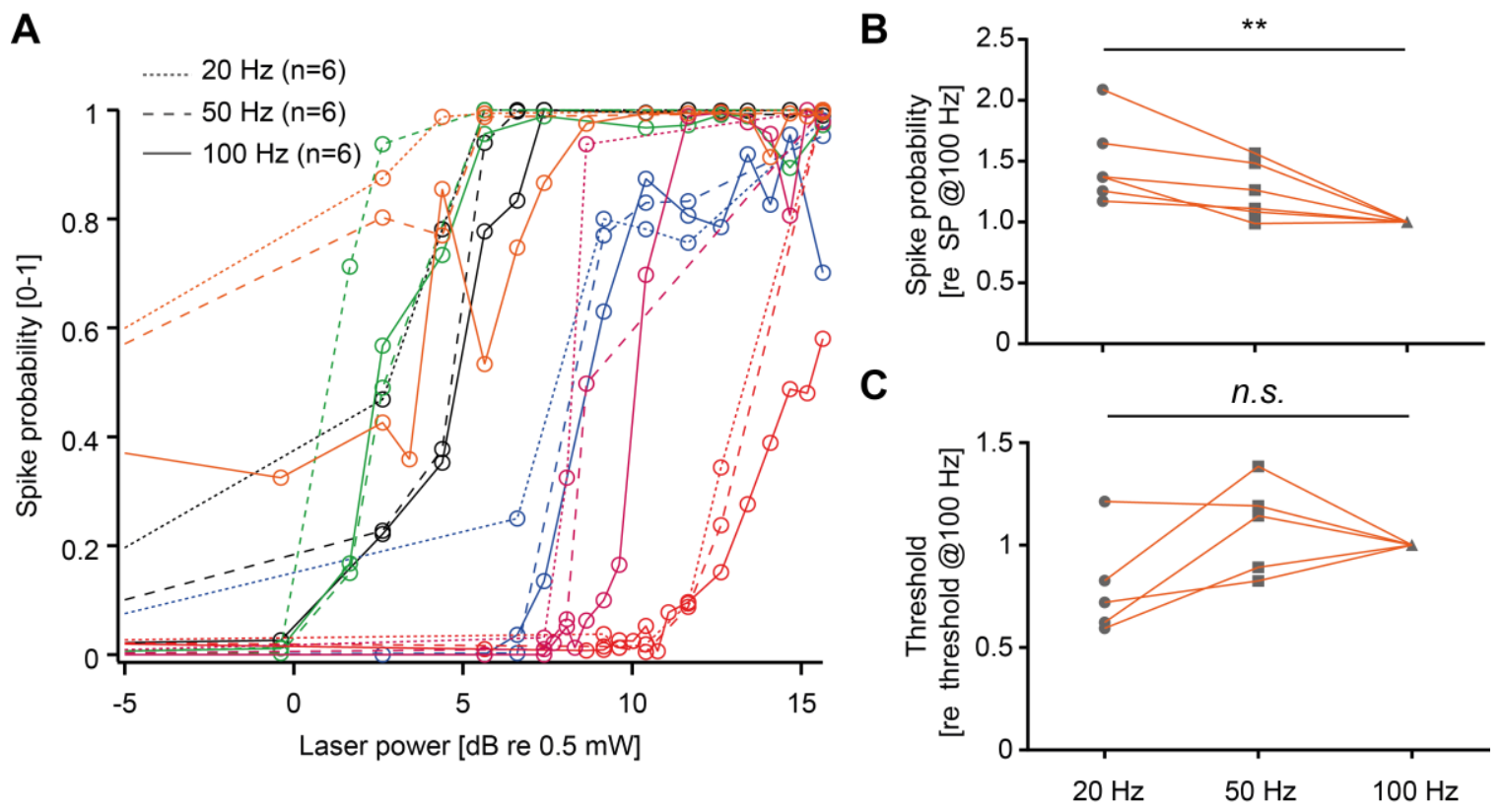

Figure 5.11: Spike fidelity increases for lower stimulation rates at the same light intensity A: Rate-level functions from a subset of optically-stimulated fibers ( $n=6$ from 2 mice, colorcoded for each SGN) at increasing light intensity and different pulse rates. Dotted lines show responses at $20 \mathrm{~Hz}$, dashed lines represent responses at $50 \mathrm{~Hz}$ and solid lines show responses at $100 \mathrm{~Hz}$. B: Ratio of average spike probability (SP) across stimulus intensities tested at 20 and $50 \mathrm{~Hz}$ over that at $100 \mathrm{~Hz}$. SGNs fire more reliably when decreasing stimulation rate from 100 to $20 \mathrm{~Hz}$ for the same light intensity levels ( $p=0.0062$; Dunn's test). C: Threshold level (light intensity eliciting $10 \%$ of the driven rate) at 20 and $50 \mathrm{~Hz}$ normalized to that at $100 \mathrm{~Hz}$. Data show that increasing pulse rate from $20-50-100 \mathrm{~Hz}$ has no significant effect on threshold level $(p=0.1644 ;$ Kruskal-Wallis test).

The extent of the growth of these firing rates, however, differ among modalities. In response to pulsatile optogenetic stimulation, the majority of fibers showed a saturated rate at maximum of one spike per pulse, with a fraction of them reaching higher values. Generation of a second spike may result from residual charge in the membrane (Miller et al., 1999). I argue that extended post-spike depolarization due to non-instantaneous $\mathrm{ChR}$ closure might trigger a doublet in SGNs with high levels of ChR expression (Gunaydin et al., 2010; Mattis et al., 2011). In contrast, there did not seem to be a hard saturation of response in acoustic click stimulation, probably due to the high thresholds observed. SGNs usually require higher intensity clicks to fire than they would need using tones at their CF (with differences that can reach $50 \mathrm{~dB}$ ). The threshold levels to clicks reported here are comparable to literature data from cat and guinea pig (Lin and Guinan Jr, 2000, 2004; Versnel et al., 1997). An important fraction of SGNs showed 
very low optogenetic thresholds $(\sim 35 \%$ with threshold lower than $1 \mathrm{~mW}$, i.e. $<1 \mu \mathrm{J})$, bringing the energy requirements close to CI levels $(0.2 \mu \mathrm{J}$ per pulse (Zierhofer et al., 1995)). Opsins that are well tolerated by the expressing cells, as f-Chrimson, are expected to exhibit high $\mathrm{ChR}$ densities in SGNs, which allows for lower thresholds and therefore, a higher spatial selectivity would be in principle possible (Schoenenberger et al., 2008). Taking into account that stimulation was performed using a single emitter placed at the round window, it is likely that thresholds can be further improved by using multiple light sources situated along the cochlear tonotopic axis and focused toward the Rosenthal's canal (Moser, 2015a; Wrobel et al., 2018).

When light pulse trains were presented at non-saturating light powers, SGNs responded preferentially by eliciting spikes at the beginning of the train. This is a different situation compared to click stimulation, where the distribution of spikes is homogeneous throughout the stimulus train. Indeed, this might result from the presence of two different opsin cycles, one that is dark-adapted and a second light-adapted cycle, whose occupancy increases with illumination time (Nikolic et al., 2009; Schneider et al., 2015). Therefore, at the beginning of the train a higher proportion of ChRs are in the dark-adapted state, and light pulses elicit large photocurrents in the dark-adapted open state of the channel, which is highly permeable to $\mathrm{H}^{+}$ and cations (Schneider et al., 2013), thus triggering spikes. As the train proceeds, an increasing number of channels shift to the light-adapted cycle, where the open state is less conductive due to the conformational change produced around the retinal binding site (Berndt et al., 2010). Modelling studies of ChR2 estimate that the light-adapted open state is 10-fold less conductive than its dark-adapted counterpart (Nikolic et al., 2009). Thus, photocurrents do not reach a threshold level and spikes do not occur (Bruun et al., 2015; Schneider et al., 2015).

Among the various types of sound stimuli, the main reason for using clicks here is their abrupt onset, similar to that of light pulses. In the classical view, clicks deposit an energy burst in the cochlea that evoke a travelling wave moving toward the apical part of the basilar membrane. When this motion reaches the tonotopic region innervated by a particular SGN, transduction and spike firing occurs (Lin and Guinan Jr, 2000). Fibers with a high CF (> $4 \mathrm{kHz}$ ), as the vast majority of SGNs in the mouse cochlea, respond to clicks with a single spike whose latency is inversely correlated to the CF of the fiber, reflecting its tonotopic position (Evans, 1972; Kiang et al., 1965; Lin and Guinan Jr, 2000). In contrast, optical stimulation directly activates lightsensitive SGNs by eliciting photocurrents that in turn open voltage-dependent $\mathrm{Na}^{+}$channels, 
what explains the shorter mean latencies found for optical stimulation. Similarly, the shorter latencies observed for electrical stimulation (Miller et al., 1999, 2006) likely reflect the faster spike generation process there. One common feature to acoustic stimulation, single-channel optogenetic and monopolar electrical stimulation is the reduction of first spike latency and jitter as stimulus levels increase. Likely, the underlying cause of this correlation in natural hearing is the increase of synaptic input at increasing sound levels, giving rise to higher amplitude and steeper rise of evoked EPSPs that, in turn, trigger spikes with shorter latency and jitter (Rutherford et al., 2012). A similar effect was observed using electric current at increasing charge and shorter rise times (Rutherford et al., 2012) and the same could apply to optogenetic stimulation. Some authors, however, suggest that in response to monopolar electrical stimulation, the migration of the effective excitation site to more central sites in the modiolus as stimulus intensity grows can also affect spike latency (Frijns et al., 1996; Miller et al., 1999; Rubinstein, 1991). Similarly, this might apply to optogenetic stimulation under high and spread ChR expression levels as it is the case with f-Chrimson (see Chapter 4), since at increasing stimulus intensity, suprathreshold levels are reached at farther distances from the source. Furthermore, excitation site migration can also account for the decrease in spike jitter with increasing stimulus level due to spike propagation through a lower number of nodes of Ranvier (Miller et al., 1999). Despite the fact that clicks and light pulses both have an almost instantaneous rise time, the temporal jitter of spike timing was greater in click-stimulated SGNs as compared to that of optogenetic stimulation, this being still about one order of magnitude lower in electrical stimulation (Miller et al., 1999). The additional ear surgery aimed at inserting the optical fiber through the round window likely underlies the higher fraction of fibers showing SR of $>1$ spike/s in acoustic experiments, which is in turn likely to cause the differences observed in spike jitter due to the presence of random firing activity. Thus, the probability of relative refractoriness membrane states increased compared to optogenetic experiments, what is also observed when comparing electrical stimulation in deafened and normal hearing ears (Miller et al., 2006). Moreover, the higher SR can also contribute to an increased population of deactivated of voltage-gated $\mathrm{Na}^{+}$channels (Rubinstein, 1995). Taken together, these factors hamper temporal fidelity by reducing spike synchronicity to the stimulus.

In the case of electrical stimulation, low pulse rates evoke a much greater degree of SGN spike synchrony compared to optogenetic or acoustic stimulation (Litvak et al., 2001; Miller et al., 2008; Zhang et al., 2007b). In natural hearing, vector strength tends to increase with stimulus 
level for 20-30 dB before reaching saturation. The observed decrease of phase locking at the highest intensities is comparable to other reports (Dreyer and Delgutte, 2006; Heil and Peterson, 2015; Johnson, 1980). Contrarily, in optogenetic stimulation, spike synchrony does not degrade with stimulus level once saturated and the response is well phase locked even at low spike probabilities (Figure 5.9). However, measures of phase locking and jitter indicate that optogenetic temporal precision seems not as deterministic as in electrical stimulation. In CI processing strategies, much higher pulse rates seek to enhance temporal uncertainty in an effort to mimic the natural process (Litvak et al., 2001; Miller et al., 2008; Rubinstein et al., 1999), which might be avoidable in the oCI.

Moreover, not only the lower temporal precision of spiking but also the fact that spike fidelity is less deterministic in optogenetic hearing can be beneficial when comparing to electrical stimulation. For the same increments in stimulus level, spike probability decreases with increasing stimulation rate (see Chapter 4 and Figure 5.11), but rates need to increase much more in electrical stimulation to produce a similar effect (Miller et al., 2008). At moderate rates, as $100 \mathrm{~Hz}$ for light pulses and $250 \mathrm{~Hz}$ for electrical stimulation, the range of stimulus intensities that produces increases in spiking is much narrower in electrical stimulation. This translates into smaller dynamic ranges, for which optogenetic hearing promises a big step forward compared to electrical hearing. In response to electrical stimulation, dynamic range of single SGNs amounts to 1-2 dB (Javel, 1990; Miller et al., 2006; Shepherd and Javel, 1997) while optogenetic stimulation allows a 3-fold wider range. Consequently, the operational range of optogenetic stimulation at the population level almost doubles that achieved with monopolar electrical stimulation $(\sim 8 \mathrm{~dB}(\mathrm{~mW})$ vs $\sim 5 \mathrm{~dB}$, respectively, estimated using the electrical CAP growth function ( $\mathrm{Hu}$ et al., 2003). This increase in operational range might arise from the heterogeneous expression levels among SGNs (Schoenenberger et al., 2008). Under these conditions, increases in stimulus intensity lead to a successive recruitment of fibers, likely more gradual in optogenetic stimulation than in response to electrical current, resulting in a more natural increase in the perceived loudness (Jeschke and Moser, 2015).

In conclusion, the characterization of optogenetic responses of the auditory nerve to increasing light power suggests a closer to physiological situation than that of electrical hearing. Future experiments should address how SGN responses change when using multiple light emitters placed in the scala tympani of larger animal models and if, indeed, a higher spatial resolution 
can be achieved with the optogenetic approach. If this is the case, and assessments of innocuous, long-lasting functional ChR expression yield positive results; the oCI would have a paved road toward clinical translation. 


\section{General discussion}

Over the last few years, several studies demonstrated that optogenetic stimulation of lightsensitive SGNs triggers activation of the auditory pathway. Either germline transgenic expression of $\mathrm{ChR} 2$, or pre-natal injections of AAVs carrying its mutant variant CatCh proved light-sensitive rendering of SGNs feasible. However, virus-mediated expression showed a heterogeneous pattern and the temporal fidelity of optogenetic stimulation was low (Hernandez et al., 2014a). Hence, the looming challenges for progress in cochlear optogenetics were the need for higher and uniform expression levels as well as faster ChR kinetics. In the present study, I described auditory nerve responses to optogenetic stimulation using the rapidly-gating and well-expressing opsins Chronos-ES/TS and f-Chrimson. Working with Chronos highlighted the key importance of tailor-made opsin sequence and AAV optimization for unfolding the potential of optogenetic tools. My results show for the first time that (1) post-natal AAVmeditated transduction of SGNs yields high and long-lasting ChR expression levels; (2) optogenetics can drive SGN firing in vivo at near physiological rates; and (3) high light sensitivity and low illumination thresholds are achievable.

\subsection{Chronos and f-Chrimson as potential candidates for clinical cochlear optogenetics}

SGNs encode temporal cues in acoustic signals at rates of few hundreds of $\mathrm{Hz}$ with submillisecond precision (Joris et al., 2004; Liberman, 1978; Strenzke et al., 2009; Taberner and Liberman, 2005). Hence, efficient optogenetic control of SGNs, "cochlear optogenetics", mainly stands on three pillars. First, the fast deactivation kinetics of ChRs that allow responses to high optical pulse rates to faithfully reproduce the natural situation. Secondly, the light sensitivity of ChRs must be high to trigger large photocurrents and to enable a lower energy consumption per pulse that can be translated into an oCI with acceptable battery life time. The third pillar involves that the optogenetic actuators used in the cochlea should preferably be activated with red-shifted light, in order to minimize scattering in the tissue and phototoxicity risks in the long term use (Jacques, 2013; Moser, 2015a).

Here, we show that an early post-natal AAV injection approach via the round window (Akil et al., 2012) dramatically enhanced functional expression of ChRs as compared to pre-natal 
injections. Using the trans-uterine approach, only 22\% of the AAV2/6-CatCh-injected animals supported oABR (Hernandez et al., 2014a). The same method yielded only 2.5\% (3/120) of oABR-positive mice using Chronos, compared to $47.06 \%(8 / 17)$ oABR-positive mice that were injected early after birth (Chapter 3, Figure 3.4 and Figure EV5). The combination of post-natal injections and the addition of trafficking sequences in the fusion protein Chronos-ES/TS further improved functional expression, reaching 97.3\% (36/37) of the animals tested. Similarly, using Chronos under the strong CAG promoter, Duarte et al. (2018) report 87.5\% (7/8 gerbils) oABRpositive animals, although expression was not limited to SGNs. In the case of f-Chrimsonexpressing mice at 8-16 weeks of age, $100 \%$ (24/24) of the animals that endured surgery showed oABRs. In older animals (37-51 weeks of age) a decrease in efficiency was observed, since the ratio of oABR-positive mice declined to $50 \%$ (7/14). Recently, a method using intra-modiolar AAV injections in adult gerbils achieved enough CatCh expression to drive oABR in $46 \%$ (44/96) of the animals; though with a lower efficiency (transduction rate $~ 30 \%$ ) and loss of SGNs ( 25\% in all cochlear turns) (Wrobel et al., 2018).

In terms of transduction efficiency in the spiral ganglion, the post-natal approach achieved more homogeneous and higher transgene expression levels compared to pre-natal AAV injections. Trans-uterine injected AAVs carrying CatCh generated a strong basoapical expression gradient, peaking at around $65 \%$ of basal SGNs and decreasing to $\sim 5 \%$ at the cochlear apex in oABRpositive mice (Hernandez et al., 2014a). A similar pattern was observed in the very few embryonic injections of AAV2/6-Chronos-EGFP that supported SGN transduction (Chapter 3, Figure 3.4B). This graded expression resulted despite the higher viral titer and volume used in the present study $\left(1.5 \mu \mathrm{l}\right.$ and $10^{12} \mathrm{GC} / \mu \mathrm{l}$ vs $0.25 \mu \mathrm{l}$ and $10^{11} \mathrm{GC} / \mu \mathrm{l}$ (Hernandez et al., 2014a)); suggesting that the method itself underlies this effect. Regardless of the AAV serotype used, early post-natal injections through the round window rendered $\mathrm{ChR}$ expression throughout the cochlea at uniform and higher levels (Chapter 3, Figure 3.4C,D,F; Chapter 4, Figure 4.4D,F and Supplementary Figure 10A-C; Chapter 5, Figure 5.3A,B). This supposed an important step forward toward the future development of the oCI, since it enables stimulation of SGNs tuned for a wide range of frequencies along the tonotopical axis in the cochlea, hence, collectively covering a wide range of frequencies (Jeschke and Moser, 2015). However, the ability to optogenetically stimulate the auditory nerve does not only rely on high numbers of transduced SGNs, but rather on the plasma membrane localization of the fusion proteins, what can explain the typical dysfunctionality observed with the original Chronos construct (Chapter 3, Figure 
3.3B and Figure EV1). Indeed, average transduction rates of $\sim 30 \%$ routinely supported oABRs (Wrobel et al., 2018). On the other hand, one noticeable shortcoming of the post-natal approach was the viral spread to the non-injected ear. This was especially significant for Chronos and Chronos-ES/TS (Chapter 3), while in animals transduced with f-Chrimson expression levels in the contralateral side were not as elevated (Chapter 4, Figure 4.4D,F and Supplementary Figure 10A,C; Chapter 5, Figure 5.3A,B). Given that both Chronos constructs used the powerful AAV variant PHP.B as a delivery vehicle, this seems to indicate that the usage of this capsid underlies the spread in ChR expression. However, injections in early post-natal stages seem to account at least for the $\sim 5 \%$ of expression found with f-Chrimson (Chapter 4, Figure 4.4D,F and Supplementary Figure 10A,C; Chapter 5, Figure 5.3A,B). Therefore, there is room for further optimization. In this direction, another report that used the same combination of AAV2/6 and the human synapsin promoter as in Chapter 4, but intra-modiolar injections at later ages, did not found measurable levels of ChR expression in the contralateral ear (Wrobel et al., 2018).

Furthermore, f-Chrimson expression was proven functional up to more than 9 months, at a time point when the characteristic age-related hearing loss of the C57BL6/J strain was already notorious (Kane et al., 2012) (Chapter 4, Supplementary Figure 10). Additional experiments showed that one mouse was oABR-responsive at 51 weeks of age, which is the latest time point post-injection that I tested (Appendix, Figure A1). Despite the aforementioned lower number of oABR-positive animals at this time point, the fraction of ChR-expressing SGNs was unaltered in responsive animals (Chapter 4, Supplementary Figure 10A,C). In oABR-negative animals, the transduction rate of SGNs was strongly reduced as compared to oABR-positive mice (median: $3.07 \%, \mathrm{n}=4$ ). Nevertheless, these results provide evidence of a long-lasting, functional $\mathrm{ChR}$ expression that is in line with transduction of cochlear nucleus neurons with ChR2 and halorhodopsin in rats (Shimano et al., 2013) and superior to the latest time point for which Duarte et al. (2018) reported positive oABRs in Chronos-injected mice (18 weeks) and to the 6 months reported for CatCh-expressing macaque retinal ganglion cells (Chaffiol et al., 2017). AAV injection or ChR expression caused no detrimental effects assessed by the unaltered SGN density, comparable hearing thresholds and normal behavior of the animals for any of the constructs tested here. In fact, safety of ChR2 expression has been evidenced over 8 months in the frontal cortex of macaques (Han et al., 2009). However, high expression levels of fluorescent soluble proteins as GFP can lead to strong immune responses, also in immune-privileged organs as the eye (Dalkara et al., 2013). Therefore, future constructs aimed at human clinical trials 
should exclude fluorescent marker proteins. Despite AAV injections were performed in an early post-natal stage, when the murine immune system is still immature, it is unlikely that this underlies the avoidance of an immune response. Intra-modiolar injections of AAV2/6-CatChEYFP in adult gerbils did not give rise to observable symptoms of immune response, and the reported loss of SGNs was rather attributable to the applied pressure as it was also observed with sham injections (Wrobel et al., 2018).

Perhaps the most remarkable achievement of this work is the enhancement on optogeneticallydriven spiking rates in SGNs. In the seminal work by Hernandez et al. (2014), responses at the population level vanished at stimulation rates below $100 \mathrm{~Hz}$ for $\mathrm{ChR} 2$, which is well below the natural sustained firing rates of SGNs (Strenzke et al., 2009). Recent work reported that CatChexpressing SGNs can perform better, eliciting responses synchronized to the stimulus up to 100 $\mathrm{Hz}$ at the single unit level (Wrobel et al., 2018). However, the superior performance of both $\mathrm{f}-$ Chrimson and Chronos-ES/TS in the spiral ganglion is evidenced by the considerable proportion of SGNs that maintain phase locked responses even at 200 and $500 \mathrm{~Hz}$, respectively (Chapter 3, Figure 3.6B,C, Chapter 4, Figure 4.6B,D). Moreover, a minimal fraction of cells producing synchronized responses might suffice to activate auditory pathway (Bourien et al., 2014), judging by the fact that oABRs remained sizeable at $200 \mathrm{~Hz}$ for CatCh (Wrobel et al., 2018), at $250 \mathrm{~Hz}$ for f-Chrimson (Chapter 4, Supplementary Figure 10G,J) and at $1 \mathrm{kHz}$ for ChronosES/TS (Chapter 3, Figure 3.5G-I). As a note of caution on the responses of Chronos-ES/TS for high stimulation rates, I add that the short averaging window did not allow for evaluation of later ABR waves, hence it cannot be concluded with certainty that the responses reported SGN compound action potentials and that activity was propagated along the pathway. In the case of Chronos-ES/TS, average synchronization estimates were in line with other reports that expressed the ChR in the cochlear nucleus (Guo et al., 2015; Hight et al., 2015). For f-Chrimson, a major leap forward in faster closing kinetics and maximal driven firing rates was achieved, as compared to the wild-type channel or its first described faster mutant ChrimsonR (Klapoetke et al., 2014). This brings red-shifted optogenetics to a new level in terms of speed, comparable to the ChR2 mutant ChETA (Gunaydin et al., 2010). In addition, results presented here indicate that both ChRs show a high temporal stationarity of efficacy in response to long pulse trains of $400 \mathrm{~ms}$. As a result of that, SGNs expressing Chronos-ES/TS or f-Chrimson are able to follow extended stimulation patterns, though this ability tends to decline at high frequencies. This is relevant with regards to the design of the future oCI since, independently of the stimulation 
strategy used, faithful representations of natural sounds would need ChRs that are responsive over extended time periods (Jeschke and Moser, 2015). Besides that, optogenetic stimulation of SGNs expressing Chronos-ES/TS or f-Chrimson not only drive spiking with sub-millisecond precision, but also typically results in the elicitation of a single spike per light pulse. This contrasts to other optogenetic actuators with longer open lifetimes as $\mathrm{CatCh}$, where $\sim 30 \%$ of CatCh-expressing SGNs responded with an average of more than 2 spikes per pulse (Wrobel et al., 2018). Hence, briefer photo-depolarization and faster recovery (Gunaydin et al., 2010) minimizes extended depolarizations and super-numerous spikes.

Another important issue for the oCI is the light sensitivity of the ChRs, which is traded off for channel kinetics in fast actuators due to their shorter open state lifetimes (Klapper et al., 2016). However, high expression levels of fast channels can lead to enhanced photocurrents in the illuminated cells, therefore counterbalancing the impact of their shorter opening. Using fChrimson, we report average energy thresholds below $1 \mu \mathrm{J}$ for far-field neural population responses that reached $0.5 \mu \mathrm{J}$ in the best case (Chapter 4, Figure 4.5C,D,G), which is by far the lowest thresholds reported so far for optogenetics in the spiral ganglion (Duarte et al., 2018; Hernandez et al., 2014a; Wrobel et al., 2018). At the single unit level, average spike thresholds amounted to $\sim 3 \mu \mathrm{J}$, but the most responsive units were already active at energy levels as low as $0.39 \mu \mathrm{J}$ (Chapter 5, Figure 5.5B,D and Figure 5.7B). This further indicates that a minimal fraction of active SGNs is able to mediate ABRs (Bourien et al., 2014) and thus, highlights the power of the population response arising from the cooperation of minimal numbers of active SGNs at reporting information in the auditory nerve (Liberman, 1978). On the other hand, due to its brief $\tau_{\text {off, }}$ Chronos-ES/TS required higher average energies to drive oABRs at $\sim 7 \mu \mathrm{J}$, in the best case reaching $\sim 1 \mu \mathrm{J}$ (Chapter 3, Figure 3.5A,B); which is a lower threshold than that reported in another study using Chronos (Duarte et al., 2018). Indeed, these thresholds are 2fold lower than the average estimates that we recorded for the original Chronos and 4-fold more sensitive than in the case of the most sensitive animal (Chapter 3, Figure 3.5A,B), but still far from the sensitivity achieved with the red-shifted opsin. Thereby, the addition of targeting sequences to Chronos improved its performance, but additional optimization of Chronos-ES/TS plasma membrane expression might lower these figures further. Hence, in regard to power consumption, these results put f-Chrimson in a better position to be the opsin of choice for the oCI, since energy requirements using it seem more assumable and, most importantly, they are close to those of the eCI $(0.2 \mu \mathrm{J}$ per pulse) (Zierhofer et al., 1995). However, it is important to 
note that projecting light from the round window is not the optimal strategy for estimating thresholds, therefore it is very likely that the values obtained here are underestimations. Instead, using an array of emitters placed in the scala tympani and facing the Rosenthal's canal would enable to drive SGN spiking using intensities in the range of few $\mu \mathrm{W}$, as estimated by computational modelling (Wrobel et al., 2018). Furthermore, behavioral experiments in gerbils showed that the light intensities required to elicit auditory percepts are 2 to 3 -fold lower than those required for evoking oABRs (Wrobel et al., 2018).

Additionally, the ability of f-Chrimson to operate with orange-red light represents yet another advantage over blue light-gated opsins. Using light of longer wavelengths to illuminate the cochlea bears two main intrinsic advantages. First, it would increase light penetration through the bone and ganglion until reaching the target SGNs, and in a similar manner scattering would be reduced (Jacques, 2013; Yizhar et al., 2011). This would enable to target specific neural populations more precisely, thereby eventually allowing to increase the number of independent frequency channels. This would be a more realistic outcome if light of longer wavelengths is used over blue light, given the physical properties of long wavelength light. Secondly, the lower energy of red-shifted photons would minimize the risk of potential phototoxicity in the tissue in the long term (Ham et al., 1976; Wu et al., 2006). Detrimental effects associated with high energy photons, as the production of reactive oxygen species leading to damage to mitochondria and DNA or apoptosis, are less likely to occur using lower energy photons (Marek et al., 2018). Moreover, since the potential of photochemical damage decays exponentially with wavelength, much higher radiant fluxes could be safely used. For applications in the retina, the safety threshold for $590 \mathrm{~nm}$ light is three orders of magnitude higher than for $470 \mathrm{~nm}$ light (European Commission, 2006; Sengupta et al., 2016). Therefore, the advantages associated to the usage of red-shifted over blue optogenetics would ease its way to clinical application (Duebel et al., 2015; Sengupta et al., 2016).

\subsection{Comparison to other strategies aimed at confronting SNHL}

Over the last two decades, research has progressed toward making available causal therapies for SNHL, but they are still lacking. Three major biological approaches to confront SNHL centralized the efforts: gene-based therapy, regenerative therapy and pharmacology (reviewed in (Géléoc and Holt, 2014; Moser, 2015b; Müller and Barr-Gillespie, 2015; Zhang et al., 2018). 
While current electrical stimulation strategies as well as future optogenetic approaches rely on prostheses, restoring the normal function of the defective sensory cells has the potential to recover the natural frequency and intensity resolution found in healthy organs (Zhang et al., 2018). In the last years, major advances have been done toward the regeneration of HCs, with one study being in phase II of a FDA-approved clinical trial (ClinicalTrials.gov, 2014; identifier: NCT02132130). Based on preclinical work (Izumikawa et al., 2005), this study aims at inducing trans-differentiation of supporting cells into HCs by adenoviral delivery of the Atohl gene, which codes for a transcription factor that regulates $\mathrm{HC}$ development. However, in a significant portion of the cases, the genetic origin of the dysfunction and loss of HCs would require not only to regenerate sensory cells, but also to suppress the function of mutant alleles or to provide wild-type copies to restore the normal function of the cells (Zhang et al., 2018). Furthermore, there are a number of open questions related to a higher risk of tumorigenesis of the reprogrammed cells as well as to reduced cell survival in a dysfunctional organ of Corti (Wang et al., 2009).

On the other hand, cochlear gene therapeutics involves the transduction of cells with wild-type genes, principally to replace defective copies and restore auditory function. Moreover, gene therapy could also be used in combination with current CIs by delivering neurotrophin vectors to promote afferent fiber growth toward the electrodes, thus improving the auditory nerve dynamic range and reducing thresholds (Pinyon et al., 2014).

Since most forms of genetic SHNL are recessive (Shearer et al., 2013) providing the target cells with a single wild-type allele could result in improved hearing (Sacheli et al., 2013). For example, a recent report (Pan et al., 2017) using a mouse model of Usher syndrome type C (affecting balance, vision and hearing) shows that ABR thresholds decreased by 60-70 dB after harmonin delivery by AAV-Anc80L65 (Zinn et al., 2015), with effects lasting for at least 6 months. The application of the Anc80L65 serotype underlies this success, given that it targets HCs with high efficiency (Suzuki et al., 2017). However, this study also pointed out that the treatment was only effective when round-window injections were performed immediately after birth.

Dominant mutations such as the Beethoven allele of the transmembrane channel-like gene family $1\left(T m c 1^{B t h}\right)$, on the other hand, can be targeted by non-viral, genome-editing approaches, such as CRISPR-Cas9. Even though the mutant allele differs from the wild-type gene only at one base pair, the strategy seems effective at restoring ABR and behavioral thresholds, reduction 
of progressive hearing loss and enhancing cell survival (Gao et al., 2018). Once more, the study was performed in neonatal mice.

These examples provide evidence about cochlear gene therapy as a powerful tool for potential clinical translation, but also highlight major hurdles that remain at the moment. First, the timing of the treatment relative to disease onset seems critical. Mutations or cochlear injury cause different patterns of cellular degeneration that advance differentially in each particular case. Nevertheless, it is a shared requirement between prosthetic approaches and gene therapy that a sufficient number of sensory cells or SGNs survive for the treatment to be successful. Importantly, genetic mutations affecting the normal cochlear development account for more than half of all cases of genetic forms of SNHL (Zhang et al., 2018). Hence, these cases would probably require prenatal human gene therapy, which is per se controversial (David and Waddington, 2012). Secondly, AAVs have a size limitation of around $5 \mathrm{~kb}$, posing a physical barrier to larger deafness genes such as those coding for the $\mathrm{Ca}^{2+}$-channel complex or otoferlin. These could, however, be overcome by using dual-vector approaches or adenovirus (Moser, 2015a). Moreover, effects of ectopic expression of the transgenes and efficacy duration of the treatments in the long term are yet to be reported (Zhang et al., 2018).

Taken together, a generalization of causal treatments for SNHL is still far from becoming a reality. Until they are available, CIs will remain the state-of-the-art to confront many different forms of SNHL, where the main constraint is the survival of a minimal number of SGNs cell bodies and central processes (Wilson and Dorman, 2008).

Hearing impairment largely ensues from damage to or loss of the delicate HCs. Particularly, in deaf cochleae IHCs are mostly or fully lacking. Design of current eCIs and future oCIs will continue to face this situation that usually brings about the degeneration of SGN peripheral processes, thus enlarging the gap between the electrodes and sites of excitation. Fortunately, at least a fraction of SGN somata survive even long after the onset of dysfunction (Miura et al., 2002), enabling the stimulation of the peripheral auditory system and partial restoration of hearing. However, the range of SGN survival among patients is wide and more reduced in specific tonotopic regions (Leake and Rebscher, 2004). The auditory nerve and ascending pathways are regarded as the biological component of the CI, since their integrity influences outcomes as much as the implant itself (Wilson and Dorman, 2008). Interestingly, no clear correlation has been found between survival of SGNs and better CI performance (Blamey, 1997; Khan et al., 2005). In contrast, there seem to be a threshold in SGN survival that allows a 
minimal function above which, the status and capabilities of the ascending pathways and auditory brain mark the difference in outcome (Wilson and Dorman, 2008). This represents a shared concern by designers of any type of CI, along with the fact that achieved outcomes seem inversely related to the duration of deafness prior to implantation (Blamey et al., 1996; Sharma et al., 2002) as a result of cross-modal plasticity (Bavelier and Neville, 2002). Additionally, the decreasing available volume in the cochlea toward the apex constitutes a challenge for achieving deep insertions of any kind of CI placed in scala tympani. Therefore, stimulation of SGNs in the low frequency region, corresponding to the pitch of human voices, is limited (Wilson and Dorman, 2008).

For the eCI, different electrode configurations have been explored, although current CIs use a monopolar configuration because it permits a performance that is as good as other types with less power requirements (Wilson and Dorman, 2008). Overlapping in SGN groups that can be stimulated by more than one electrode is one of the main issues in the eCI, since this reduces the number of channels that independently convey information to the brain. Furthermore, good spatial specificity of stimulation is hindered by the relatively long distance between the electrodes and SGNs. Placement of implants closer to the Rosenthal's canal mildly improve spatial specificity (Cohen et al., 2006), but a new implant design as the oCI holds a much larger potential improvement in the number of independent channels (Jeschke and Moser, 2015). Another preclinical alternative to conventional implants aims at using intramodiolar electrodes directly placed on the auditory nerve to stimulate the SGNs central axons. Preliminary reports on this approach show lower thresholds and reduced spread of excitation compared to scala tympani stimulation (Badi et al., 2003; Middlebrooks and Snyder, 2007).

Despite the great limitation in number of independent stimulation sites, the performance achieved with the eCI is remarkable, even enabling fluent telephone communication for many users (Wilson, 2018), although the variability among subjects is high (Helms et al., 1997). It is worth noting that CI systems do not replicate many processes that occur in natural hearing upon sound stimulation in order to enable speech understanding (i.e. the role of efferent feedback on OHCs (Guinan, 2010) or the presence of spontaneous activity in the auditory nerve (Miller et al., 2006)). Ingenious processing strategies contribute to these outcomes, i.e. interleaving temporal stimulation among electrodes to reduce interaction (continuous interleaved sampling, (CIS) strategy (Wilson et al., 1991)) or scanning the sound signals to activate electrodes that 
correspond to the frequencies with the highest amplitudes ( $n$-of- $m$, advanced combinational encoder (ACE) and spectral-peak (SPEAK) strategies (Kiefer et al., 2001; Skinner et al., 1994; Wilson et al., 1988)). These strategies are focused on encoding the envelope of sound signals by modulating the amplitude of constant rate pulse trains at particular electrodes, which is important for speech understanding with eight or less channels, as it is typically the case for the eCI (Smith et al., 2002). Nevertheless, the fine structure of the signals also contributes to speech reception in noise, while it conveys the practical totality of music information in this channel range (Nie et al., 2005; Smith et al., 2002). A faithful representation of the fine structure information is well limited by the restriction in the number of independent stimulation channels, since this impedes a fine-tuned stimulation of small SGN groups. Processing strategies as the fine structure processing (FSP) have been postulated to provide advantages over envelopefocused strategies (Arnoldner et al., 2007) but there is no consensus on the matter (Magnusson, 2011). Additionally, differential current steering between electrode pairs can create "phantom channels" to increase the number of discernable pitches, thereby improving music appreciation and speech in noise recognition (Firszt et al., 2007; Koch et al., 2007), at the cost of higher energy requirements (Landsberger and Srinivasan, 2009). However, outcomes are not always significantly different from classical approaches (Nogueira et al., 2015; Wilson and Dorman, 2008). Since the 1990s the performance of unilateral CIs has reached a plateau and only the adjunctive stimulation has lately advanced (Wilson, 2018). Some patients that retain small populations of IHCs in the low frequency region can benefit from combined electric and acoustic stimulation (EAS approach), thereby improving their auditory perception (Dorman et al., 2008, 2015). Together with EAS, bilateral implantation provides advantages in non-optimal listening situations as well as improving sound localization, which has recently boosted its application (Gifford et al., 2015). Furthermore, recent evidence suggests that deactivating the electrodes that are farther away to the target SGNs or the ones that produce the greatest interactions can improve speech reception (Labadie et al., 2016). Still, the perceptual gap between CI users and normal hearing individuals remains significant at daily situations where background noise is present and at music reception (Wilson, 2018). Novel strategies aimed at reducing the gap between the electrode array and the target neurons employ neurotrophins to promote neurite growth toward the implant, achieving lower stimulation thresholds and potentially a more selective stimulation (Pinyon et al., 2014; Senn et al., 2017). 
Taken together, the usage of electrical stimulation in the cochlea inherently poses a great limitation regarding channel interaction. For prostheses placed in the very conductive perilymph, a radical reduction in channel interaction entails an essentially different stimulus modality. An increase in the number of independent stimulation channels, even if modest, would provide a qualitative improvement in hearing perception for all CI users (Fu and Nogaki, 2005; Wilson, 2018). Using light for optogenetic stimulation in the oCI, together with the high light sensitivity of the ChRs described here, would help to dramatically increase the number of independent stimulation channels. Thereby, the oCI would provide a more favorable starting point for the development of processing strategies where many more channels would potentially be available, hence easing the encoding of the fine structure of signals, enhancing pitch perception and thus improving the perception of music and speech in non-optimal conditions (Jeschke and Moser, 2015). Moreover, the extended dynamic range of the oCI (Chapter 5, Figure 5.6C) compared to the eCI would likely be critical in situations involving soft sounds and noisy environments (Spahr et al., 2007).

Optical approaches to drive SGN activity not relying on heterologous protein expression have also been suggested. Infrared neural stimulation (INS) involves direct stimulation of neurons in their native state using pulsed infrared laser light (Izzo et al., 2006; Wells et al., 2007). INS showed a higher spatial selectivity and dynamic range than that of monopolar electrical stimulation (Izzo et al., 2007; Richter et al., 2011). Spatial selectivity of INS was similar to that of optogenetic and acoustic stimulation using pure tones (Hernandez et al., 2014a). However, the mechanism of INS remains controversial. Measurements in HEK cells and Xenopus laevis oocytes suggested that INS arises from a photothermal effect that locally alters the plasma membrane capacitance and results in depolarization of the target cell (Shapiro et al., 2012). However, light stimulation well above the observed threshold in vivo evokes depolarizations that might be too weak to trigger APs (Chernov and Roe, 2014; Rettenmaier et al., 2014). Alternative potential mechanisms of action of INS comprise an energy absorption by water leading to a mild, local temperature increase in the tissue (Wells et al., 2007) or triggering the activation of TRPV heat-sensitive channels that are expressed in sensory neurons (Albert et al., 2012). Nevertheless, responses to INS in the cochlea are more likely produced by an optoacoustic effect in vivo (heat waves produced by laser pulses inducing deflection of IHCs stereocilia) (Kallweit et al., 2016; Teudt et al., 2011), given the absence of light-driven neural 
activity in deafened animals (Schultz et al., 2012; Thompson et al., 2015; Verma et al., 2014) which dismisses INS as a suitable road to confront SNHL. Furthermore, the application of visible light in optogenetics comparatively reduces potential detrimental effects of heat in the tissue (Yizhar et al., 2011); and threshold energy requirements for evoking CAP responses using INS are $~ 1-2$ orders of magnitude higher (Izzo et al., 2008) than for optogenetic stimulation (Hernandez et al., 2014a).

\subsection{Future work}

Results I presented in this work show an improved temporal bandwidth of coding in the cochlea using fast ChRs, thereby overcoming a major obstacle on the road of cochlear optogenetics toward the clinic. However, much remains to be done prior to the clinical testing of cochlear optogenetics and a suitable optoprosthesis. Future experiments need to address three major challenges: (1) long-term safety and reliability of ChR expression in SGNs; (2) efficiency, reliability and safety of oCIs; and (3) demonstration of significant advantage of the oCI over the eCI (Jeschke and Moser, 2015).

An assessment of long-term safety of ChR expression involves both the AAV and the heterologous protein chosen as an optogenetic actuator. Recombinant AAVs are unable to replicate and do not integrate into the host genome at specific loci in the absence of Rep proteins (Smith, 2008). Instead, transgenes remain as episomes in the host cell nucleus (Colella et al., 2017; Naso et al., 2017). This contributes to long-term expression in non-dividing cells as SGNs as well as to the lack of tumorigenesis associated to AAVs (Mueller and Flotte, 2008). Gene therapy in the liver exemplifies the ability of AAVs to mediate transgene expression in the long term. Following a single application, expression was stable and episomal over 5 years in the liver of adult non-human primates (Nathwani et al., 2011). Similar assessments in the cochlea are underway in our laboratory. Preexisting antibodies against AAVs might prevent patients from taking part in clinical trials. Hence, exosome-associated AAV particles (vexosomes) have been proposed to avoid these potential immune responses, with recent studies reporting promising results at the retina (Wassmer et al., 2017) and HCs (György et al., 2017). AAV safety depends on the dose (Ramachandran et al., 2017) and at the same time, optogenetic stimulation thresholds are inversely related to ChR expression (Schoenenberger et al., 2008). Therefore, an evaluation of the viral doses and construct optimization required to elicit optogenetic responses 
in the cochlea needs to be carried out in non-human primates and covering extended periods of time. In a similar manner, a recent report studied immunological reactions and optogenetic responses in the retina of macaques over six months, using different promoters to drive CatCh expression at varying viral doses (Chaffiol et al., 2017). The study concluded that the injection procedure - and not the AAV/transgene applied — caused transient, mild inflammatory signs and that CatCh-driven responses can be triggered using safe light intensities only if the chosen promoter drove high level of ChR expression. Furthermore, this future evaluation should also assess ectopic transgene expression and track cell morphology changes that have been sometimes associated with chronic ChR expression (Miyashita et al., 2013).

Here, ChRs are specifically targeted to SGNs by means of a topical application of AAVs and by using the hSyn promoter. Higher spatial selectivity of stimulation could be achieved by using AAV capsids designed for enhanced transduction of the peripheral nervous system, as AAV PHP.S (Chan et al., 2017) and by subcellular targeting of ChRs (Grubb and Burrone, 2010; Lewis et al., 2009). Studies show preferential ChR expression at the axon initial segment by adding the ankyrinG-binding loop of voltage-gated sodium channels to the intracellular domain of ChRs (Grubb and Burrone, 2010) or to the somatodendritic compartment of neurons by attaching the melanophilin myosin-binding domain to the transgene (Lewis et al., 2009). It is possible that heterogeneous $\mathrm{ChR}$ expression levels in SGNs, resulting solely from AAV transduction (Towne et al., 2009), are sufficient to achieve a dynamic range comparable to natural hearing in response to stimulation using scala tympani light emitters. Nevertheless, if functional expression is similarly high among SGNs, differences in expression levels could be enhanced by using promoters of different strength or gene-switches at the cost of an increased approach complexity (Jeschke and Moser, 2015; Maddalena et al., 2013; Xu et al., 2001).

Furthermore, the discovery and development of optogenetic actuators with improved properties advances constantly. Characterizing vf-Chrimson (Chapter 4) in the auditory system holds the promise to bring the temporal resolution of red-shifted optogenetics closer to that achieved with Chronos, due to its faster kinetic properties compared to f-Chrimson. In addition, at the time that this thesis was written, a new Chrimson mutant was disclosed (ChrimsonSA) featuring both fast closing kinetics and an action spectrum that is $20 \mathrm{~nm}$ more red-shifted than its wild-type counterpart (Oda et al., 2018). 
In regard to the technological development toward the oCI, current experiments are evaluating different designs and prototypes. Two main categories of optical devices have been devised: active and passive implants. In active optoelectronic devices, light is generated in situ while passive implants use external light generators and waveguides to deliver it into the cochlea (Dombrowski et al., 2018; Jeschke and Moser, 2015). Nowadays, benefits and drawbacks of either category of implants seem traded in one another. Since active implants do not rely on light carriers, the process of light transmission would be spared resulting in important gains in terms of efficiency. On the other hand, light emitters need to be miniaturized and encapsulated to stabilize light sources and to allow long-term function in the perilymph-filled scala tympani. It is important to note that the eCI, as a benchmark for the oCI, is stable for decades (Lenarz et al., 2012). Furthermore, the heat generated by intracochlear emitters needs to be considered and the extent of thermal damage risk needs to be assessed (Dombrowski et al., 2018). Recently, arrays of micrometer-scale emitters ( $\mu$ LED) have been developed (Goßler et al., 2014) and there is an active research field aimed at improving current $\mu$ LED technology. Microlenses or microprisms can focus the light from $\mu$ LEDs and thereby reduce energy requirements compared to the spatially broad emission pattern of naked emitters (Chia and Levene, 2009; Choi et al., 2005; Ee et al., 2009). However, the addition of these elements would increase the rigidity of an implant intended to be placed in a spiral structure. Alternatively, vertical-cavity surface emitting lasers (VCSELs) deliver highly convergent light beams and can be miniaturized (Liao et al., 2012; Lu et al., 2009). Nevertheless, there is still much room for development of efficient light emission in the yellow-orange spectrum for red-shifted optogenetics, in the case of both $\mu$ LEDs and VCSELs (Dombrowski et al., 2018; Ledentsov et al., 2018). Passive implants offer less restrictions in terms of size of the light generator since it is located outside the cochlea, and, associated to this, the heat derived from light production do not diffuse inside the cochlea. Moreover, electrical artifacts would be of least relevance using passive devices (Moser, 2015). However, significant loss of delivered light can result from coupling and transmission, which depends on the waveguide length (Jeschke and Moser, 2015). Application of optical fibers in the cochlea is also limited by their physical properties and the angles of bending that they can endure (Balster et al., 2014). Furthermore, in this case it is crucial that the waveguides are stable for reliable light delivery in the long term (Dombrowski et al., 2018). Eventually, careful assessment needs to convey the choice of the optimal oCI design to be further tested for longterm biocompatibility in chronically-implanted animals (Jeschke and Moser, 2015). Modelling 
studies of light spread in the cochlea and physiological estimates of spread of excitation would help designing optimized light emitters (Dombrowski et al., 2018). Importantly, emitters need to deliver light intensities not only enough to activate but to selectively recruit increasing numbers of SGNs (Jeschke and Moser, 2015). In either case, 3D imaging techniques as phasecontrast $\mathrm{x}$-ray tomography can provide insight on available luminal space in the cochlea for careful oCI design and therefore prevent tissue damage upon implant insertion (Töpperwien et al., 2018). Usage of shape memory materials can bring emitters closer to stimulation sites (modiolus-hugging arrays), thereby enhancing oCI efficiency (Min et al., 2013). Furthermore, new coding strategies would need to be developed for multichannel oCIs (Dombrowski et al., 2018; Moser, 2015a). It might as well be desirable that the first oCIs designed for human use combine electrical and optical stimulation, so that electrical stimulation serves as a backup strategy (Jeschke and Moser, 2015).

Finally, it is of capital importance to demonstrate that optogenetic stimulation actually implies a major perceptual advantage over current electrical stimulation strategies, which constitute a strong benchmark (Wilson and Dorman, 2008). Future experiments will need to compare energy requirements, frequency and intensity resolution in electrical and optogenetic hearing. Primary physiological assessments on this matter are underway in our laboratory using single- and multiunit recordings in the auditory pathway of rodents. Importantly, an increase in stimulation channels should not trade resolution off for energy consumption (Dombrowski et al., 2018). Preclinical studies in rodents, non-human primates and humans should then test these hypotheses at the behavioral and perceptual level (Jeschke and Moser, 2015; Moser, 2015a). If these requirements are met, a new stimulation modality would be available for patients who can benefit from improved hearing restoration. 


\section{References}

Adamantidis, A., Arber, S., Bains, J.S., Bamberg, E., Bonci, A., Buzsáki, G., Cardin, J.A., Costa, R.M., Dan, Y., Goda, Y., et al. (2015). Optogenetics: 10 years after ChR2 in neurons--views from the community. Nat. Neurosci. 18, 1202-1212.

Adamantidis, A.R., Zhang, F., Aravanis, A.M., Deisseroth, K., and Lecea, L. de (2007). Neural substrates of awakening probed with optogenetic control of hypocretin neurons. Nature 450, 420-424.

Akil, O., Seal, R.P., Burke, K., Wang, C., Alemi, A., During, M., Edwards, R.H., and Lustig, L.R. (2012). Restoration of Hearing in the VGLUT3 Knockout Mouse Using Virally Mediated Gene Therapy. Neuron 75, 283-293.

Albert, E.S., Bec, J.M., Desmadryl, G., Chekroud, K., Travo, C., Gaboyard, S., Bardin, F., Marc, I., Dumas, M., Lenaers, G., et al. (2012). TRPV4 channels mediate the infrared laser-evoked response in sensory neurons. J. Neurophysiol. 107, 3227-3234.

Aravanis, A.M., Wang, L.-P., Zhang, F., Meltzer, L.A., Mogri, M.Z., Schneider, M.B., and Deisseroth, K. (2007). An optical neural interface: in vivo control of rodent motor cortex with integrated fiberoptic and optogenetic technology. J Neural Eng 4, S143-S156.

Arnoldner, C., Riss, D., Brunner, M., Durisin, M., Baumgartner, W.-D., and Hamzavi, J.-S. (2007). Speech and music perception with the new fine structure speech coding strategy: preliminary results. Acta Otolaryngol. (Stockh.) 127, 1298-1303.

Ashmore, J. (2008). Cochlear Outer Hair Cell Motility. Physiol. Rev. 88, 173-210.

Avissar, M., Wittig, J.H., Saunders, J.C., and Parsons, T.D. (2013). Refractoriness Enhances Temporal Coding by Auditory Nerve Fibers. J. Neurosci. 33, 7681-7690.

Badi, A.N., Kertesz, T.R., Gurgel, R.K., Shelton, C., and Normann, R.A. (2003). Development of a novel eighth-nerve intraneural auditory neuroprosthesis. The Laryngoscope 113, 833-842.

Baig, S.M., Koschak, A., Lieb, A., Gebhart, M., Dafinger, C., Nürnberg, G., Ali, A., Ahmad, I., Sinnegger-Brauns, M.J., Brandt, N., et al. (2011). Loss of Ca(v)1.3 (CACNA1D) function in a human channelopathy with bradycardia and congenital deafness. Nat. Neurosci. $14,77-84$.

Balster, S., Wenzel, G.I., Warnecke, A., Steffens, M., Rettenmaier, A., Zhang, K., Lenarz, T., and Reuter, G. (2014). Optical cochlear implant: evaluation of insertion forces of optical fibres in a cochlear model and of traumata in human temporal bones. Biomed. Tech. Eng. 59, 19-28.

Barbour, D.L. (2011). Intensity-Invariant Coding in the Auditory System. Neurosci. Biobehav. Rev. 35, 2064-2072.

Bavelier, D., and Neville, H.J. (2002). Cross-modal plasticity: where and how? Nat. Rev. Neurosci. 3, 443-452.

Becker, L., Schnee, M.E., Niwa, M., Sun, W., Maxeiner, S., Talaei, S., Kachar, B., Rutherford, M.A., and Ricci, A.J. (2018). The presynaptic ribbon maintains vesicle populations at the hair cell afferent fiber synapse. ELife 7, e30241.

Berndt, A., and Deisseroth, K. (2015). Expanding the optogenetics toolkit. Science 349, 590-591.

Berndt, A., Yizhar, O., Gunaydin, L.A., Hegemann, P., and Deisseroth, K. (2008). Bi-stable neural state switches. Nat. Neurosci. 12, 229-234. 
Berndt, A., Prigge, M., Gradmann, D., and Hegemann, P. (2010). Two open states with progressive proton selectivities in the branched channelrhodopsin-2 photocycle. Biophys. J. 98, 753.

Berndt, A., Schoenenberger, P., Mattis, J., Tye, K.M., Deisseroth, K., Hegemann, P., and Oertner, T.G. (2011). High-efficiency channelrhodopsins for fast neuronal stimulation at low light levels. Proc. Natl. Acad. Sci. 108, 7595-7600.

Berndt, A., Lee, S.Y., Ramakrishnan, C., and Deisseroth, K. (2014). Structure-Guided Transformation of Channelrhodopsin into a Light-Activated Chloride Channel. Science 344, 420-424.

Blamey, P. (1997). Are spiral ganglion cell numbers important for speech perception with a cochlear implant? Am. J. Otol. 18, S11-12.

Blamey, P., Arndt, P., Bergeron, F., Bredberg, G., Brimacombe, J., Facer, G., Larky, J., Lindström, B., Nedzelski, J., Peterson, A., et al. (1996). Factors affecting auditory performance of postlinguistically deaf adults using cochlear implants. Audiol. Neurootol. 1, 293-306.

Bourien, J., Tang, Y., Batrel, C., Huet, A., Lenoir, M., Ladrech, S., Desmadryl, G., Nouvian, R., Puel, J.-L., and Wang, J. (2014). Contribution of auditory nerve fibers to compound action potential of the auditory nerve. J. Neurophysiol. 112, 1025-1039.

Boyden, E.S., Zhang, F., Bamberg, E., Nagel, G., and Deisseroth, K. (2005). Millisecond-timescale, genetically targeted optical control of neural activity. Nat Neurosci 8, 1263-1268.

Brandt, A., Striessnig, J., and Moser, T. (2003). CaV1. 3 channels are essential for development and presynaptic activity of cochlear inner hair cells. J. Neurosci. 23, 10832-10840.

Brandt, A., Khimich, D., and Moser, T. (2005). Few CaV1. 3 channels regulate the exocytosis of a synaptic vesicle at the hair cell ribbon synapse. J. Neurosci. 25, 11577.

Brigande, J.V., Gubbels, S.P., Woessner, D.W., Jungwirth, J.J., and Bresee, C.S. (2009). Electroporation-mediated gene transfer to the developing mouse inner ear. Methods Mol. Biol. 493, 125139.

Brown, M.C. (1987). Morphology of labeled afferent fibers in the guinea pig cochlea. J. Comp. Neurol. 260, 591-604.

Bruun, S., Stoeppler, D., Keidel, A., Kuhlmann, U., Luck, M., Diehl, A., Geiger, M.-A., Woodmansee, D., Trauner, D., Hegemann, P., et al. (2015). Light-Dark Adaptation of Channelrhodopsin Involves Photoconversion between the all-trans and 13-cis Retinal Isomers. Biochemistry 54, 5389-5400.

Buran, B.N., Strenzke, N., Neef, A., Gundelfinger, E.D., Moser, T., and Liberman, M.C. (2010). Onset coding is degraded in auditory nerve fibers from mutant mice lacking synaptic ribbons. J. Neurosci. Off. J. Soc. Neurosci. 30, 7587-7597.

Busskamp, V., Picaud, S., Sahel, J.A., and Roska, B. (2012). Optogenetic therapy for retinitis pigmentosa. Gene Ther. 19, 169-175.

Buzsáki, G. (2015). Hippocampal sharp wave-ripple: A cognitive biomarker for episodic memory and planning. Hippocampus 25, 1073-1188.

Cariani, P.A., and Delgutte, B. (1996). Neural correlates of the pitch of complex tones. I. Pitch and pitch salience. J. Neurophysiol. 76, 1698.

Chaffiol, A., Caplette, R., Jaillard, C., Brazhnikova, E., Desrosiers, M., Dubus, E., Duhamel, L., Macé, E., Marre, O., Benoit, P., et al. (2017). A New Promoter Allows Optogenetic Vision Restoration with Enhanced Sensitivity in Macaque Retina. Mol. Ther. 25, 2546-2560. 
Chan, K.Y., Jang, M.J., Yoo, B.B., Greenbaum, A., Ravi, N., Wu, W.-L., Sánchez-Guardado, L., Lois, C., Mazmanian, S.K., Deverman, B.E., et al. (2017). Engineered AAVs for efficient noninvasive gene delivery to the central and peripheral nervous systems. Nat. Neurosci. 20, 1172-1179.

Chernov, M., and Roe, A.W. (2014). Infrared neural stimulation: a new stimulation tool for central nervous system applications. Neurophotonics 1, 011011.

Chia, T.H., and Levene, M.J. (2009). Microprisms for In Vivo Multilayer Cortical Imaging. J. Neurophysiol. 102, 1310-1314.

Choi, H.W., Gu, E., Liu, C., Girkin, J.M., and Dawson, M.D. (2005). Fabrication and evaluation of GaN negative and bifocal microlenses. J. Appl. Phys. 97, 063101.

ClinicalTrials.gov (2014). Safety, Tolerability and Efficacy for CGF166 in Patients With Bilateral Severe-to-profound Hearing Loss. Identifier: NCT02132130.

ClinicalTrials.gov (2015). RetroSense Therapeutics Phase I/II Clinical Trial (RST-001) Identifier: NCT02556736.

ClinicalTrials.gov (2017). A Phase 1/2a, Open-Label, Non-Randomized, Dose-Escalation Study to Evaluate the Safety and Tolerability of GS030 in Subjects With Retinitis Pigmentosa. Identifier: NCT03326336.

Cohen, L.T., Saunders, E., Knight, M.R., and Cowan, R.S.C. (2006). Psychophysical measures in patients fitted with Contour and straight Nucleus electrode arrays. Hear. Res. 212, 160-175.

Colella, P., Ronzitti, G., and Mingozzi, F. (2017). Emerging Issues in AAV-Mediated In Vivo Gene Therapy. Mol. Ther. Methods Clin. Dev. 8, 87-104.

Colletti, L., Shannon, R., and Colletti, V. (2012). Auditory brainstem implants for neurofibromatosis type 2: Curr. Opin. Otolaryngol. Head Neck Surg. 20, 353-357.

Dalkara, D., Byrne, L.C., Klimczak, R.R., Visel, M., Yin, L., Merigan, W.H., Flannery, J.G., and Schaffer, D.V. (2013). In Vivo-Directed Evolution of a New Adeno-Associated Virus for Therapeutic Outer Retinal Gene Delivery from the Vitreous. Sci. Transl. Med. 5, 189ra76-189ra76.

Dalkara, D., Goureau, O., Marazova, K., and Sahel, J.-A. (2016). Let There Be Light: Gene and Cell Therapy for Blindness. Hum. Gene Ther. 27, 134-147.

Dallos, P. (1996). The Cochlea (Springer Verlag, New York, Inc).

Dallos, P. (2008). Cochlear amplification, outer hair cells and prestin. Curr. Opin. Neurobiol. 18, 370376.

David, A.L., and Waddington, S.N. (2012). Candidate diseases for prenatal gene therapy. Methods Mol. Biol. Clifton NJ 891, 9-39.

Deisseroth, K. (2010). Optogenetics. Nat. Methods 8, 26-29.

Deisseroth, K., and Hegemann, P. (2017). The form and function of channelrhodopsin. Science 357.

Deisseroth, K., Feng, G., Majewska, A.K., Miesenböck, G., Ting, A., and Schnitzer, M.J. (2006). Nextgeneration optical technologies for illuminating genetically targeted brain circuits. J. Neurosci. 26, 10380.

Deverman, B.E., Pravdo, P.L., Simpson, B.P., Kumar, S.R., Chan, K.Y., Banerjee, A., Wu, W.-L., Yang, B., Huber, N., Pasca, S.P., et al. (2016). Cre-dependent selection yields AAV variants for widespread gene transfer to the adult brain. Nat. Biotechnol. 34, 204-209. 
Dombrowski, T., Rankovic, V., and Moser, T. (2018). Toward the Optical Cochlear Implant. Cold Spring Harb. Perspect. Med. a033225.

Dorman, M.F., Gifford, R.H., Spahr, A.J., and McKarns, S.A. (2008). The benefits of combining acoustic and electric stimulation for the recognition of speech, voice and melodies. Audiol. Neurootol. 13, 105112.

Dorman, M.F., Cook, S., Spahr, A., Zhang, T., Loiselle, L., Schramm, D., Whittingham, J., and Gifford, R. (2015). Factors constraining the benefit to speech understanding of combining information from lowfrequency hearing and a cochlear implant. Hear. Res. 322, 107-111.

Dreyer, A., and Delgutte, B. (2006). Phase locking of auditory-nerve fibers to the envelopes of highfrequency sounds: implications for sound localization. J. Neurophysiol. 96, 2327-2341.

Duan, M., Venail, F., Spencer, N., and Mezzina, M. (2004). Treatment of peripheral sensorineural hearing loss. Gene Ther. 11, S51-S56.

Duarte, M.J., Kanumuri, V.V., Landegger, L.D., Tarabichi, O., Sinha, S., Meng, X., Hight, A.E., Kozin, E.D., Stankovic, K.M., Brown, M.C., et al. (2018). Ancestral Adeno-Associated Virus Vector Delivery of Opsins to Spiral Ganglion Neurons: Implications for Optogenetic Cochlear Implants. Mol. Ther. 26, 1931-1939.

Duebel, J., Marazova, K., and Sahel, J.-A. (2015). Optogenetics. Curr. Opin. Ophthalmol. 26, 226-232.

Dynes, S.B.., and Delgutte, B. (1992). Phase-locking of auditory-nerve discharges to sinusoidal electric stimulation of the cochlea. Hear. Res. 58, 79-90.

Ee, Y.-K., Kumnorkaew, P., Arif, R.A., Tong, H., Gilchrist, J.F., and Tansu, N. (2009). Light extraction efficiency enhancement of InGaN quantum wells light-emitting diodes with polydimethylsiloxane concave microstructures. Opt. Express 17, 13747.

European Commission (2006). Directive 2006/25/EC of the European Parliament and of the Council (artificial optical radiation).

Evans, E.F. (1972). The frequency response and other properties of single fibres in the guinea-pig cochlear nerve. J. Physiol. 226, 263-287.

Evans, E.F. (1981). The Dynamic Range Problem: Place and Time Coding at the Level of Cochlear Nerve and Nucleus. In Neuronal Mechanisms of Hearing, J. Syka, and L. Aitkin, eds. (Boston, MA: Springer US), pp. 69-85.

Feldbauer, K., Zimmermann, D., Pintschovius, V., Spitz, J., Bamann, C., and Bamberg, E. (2009). Channelrhodopsin-2 is a leaky proton pump. Proc. Natl. Acad. Sci. 106, 12317-12322.

Fettiplace, R. (2017). Hair Cell Transduction, Tuning, and Synaptic Transmission in the Mammalian Cochlea. Compr. Physiol. 7, 1197-1227.

Fettiplace, R., and Hackney, C.M. (2006). The sensory and motor roles of auditory hair cells. Nat. Rev. Neurosci. 7, 19-29.

Fioretti, A., Poli, O., Varakliotis, T., and Eibenstein, A. (2014). Hearing Disorders and Sensorineural Aging.

Firszt, J.B., Koch, D.B., Downing, M., and Litvak, L. (2007). Current Steering Creates Additional Pitch Percepts in Adult Cochlear Implant Recipients: Otol. Neurotol. 28, 629-636. 
Flores, E.N., Duggan, A., Madathany, T., Hogan, A.K., Márquez, F., Kumar, G., Seal, R., Edwards, R., Liberman, M.C., and García-Añoveros, J. (2015). A Non-canonical Pathway from Cochlea to Brain Signals Tissue-damaging Noise. Curr. Biol. CB 25, 606-612.

Frank, T., Khimich, D., Neef, A., and Moser, T. (2009). Mechanisms contributing to synaptic Ca2+ signals and their heterogeneity in hair cells. Proc. Natl. Acad. Sci. 106, 4483.

Frank, T., Rutherford, M.A., Strenzke, N., Neef, A., Pangršič, T., Khimich, D., Fejtova, A., Gundelfinger, E.D., Liberman, M.C., Harke, B., et al. (2010). Bassoon and the synaptic ribbon organize $\mathrm{Ca}^{2}+$ channels and vesicles to add release sites and promote refilling. Neuron 68, 724-738.

Friesen, L.M., Shannon, R.V., Baskent, D., and Wang, X. (2001). Speech recognition in noise as a function of the number of spectral channels: Comparison of acoustic hearing and cochlear implants. J. Acoust. Soc. Am. 110, 1150.

Frijns, J.H., de Snoo, S.L., and ten Kate, J.H. (1996). Spatial selectivity in a rotationally symmetric model of the electrically stimulated cochlea. Hear. Res. 95, 33-48.

Froud, K.E., Wong, A.C.Y., Cederholm, J.M.E., Klugmann, M., Sandow, S.L., Julien, J.-P., Ryan, A.F., and Housley, G.D. (2015). Type II spiral ganglion afferent neurons drive medial olivocochlear reflex suppression of the cochlear amplifier. Nat. Commun. 6, 7115.

Fu, Q.-J., and Nogaki, G. (2005). Noise Susceptibility of Cochlear Implant Users: The Role of Spectral Resolution and Smearing. JARO J. Assoc. Res. Otolaryngol. 6, 19-27.

Fuchs, P.A. (2005). Time and intensity coding at the hair cell's ribbon synapse. J. Physiol. 566, 7-12.

Fuchs, P.A., Glowatzki, E., and Moser, T. (2003). The afferent synapse of cochlear hair cells. Curr. Opin. Neurobiol. 13, 452-458.

Furman, A.C., Kujawa, S.G., and Liberman, M.C. (2013). Noise-induced cochlear neuropathy is selective for fibers with low spontaneous rates. J. Neurophysiol. 110, 577-586.

Gao, X., Tao, Y., Lamas, V., Huang, M., Yeh, W.-H., Pan, B., Hu, Y.-J., Hu, J.H., Thompson, D.B., Shu, Y., et al. (2018). Treatment of autosomal dominant hearing loss by in vivo delivery of genome editing agents. Nature 553, 217-221.

Geisler, C.D., Deng, L., and Greenberg, S.R. (1985). Thresholds for primary auditory fibers using statistically defined criteria. J. Acoust. Soc. Am. 77, 1102-1109.

Géléoc, G.S.G., and Holt, J.R. (2014). Sound strategies for hearing restoration. Science 344, 1241062.

von Gersdorff, H., Vardi, E., Matthews, G., and Sterling, P. (1996). Evidence that vesicles on the synaptic ribbon of retinal bipolar neurons can be rapidly released. Neuron 16, 1221-1227.

Gifford, R.H., Driscoll, C.L.W., Davis, T.J., Fiebig, P., Micco, A., and Dorman, M.F. (2015). A WithinSubject Comparison of Bimodal Hearing, Bilateral Cochlear Implantation, and Bilateral Cochlear Implantation With Bilateral Hearing Preservation: High-Performing Patients. Otol. Neurotol. Off. Publ. Am. Otol. Soc. Am. Neurotol. Soc. Eur. Acad. Otol. Neurotol. 36, 1331-1337.

Giraudet, F., and Avan, P. (2012). Auditory neuropathies: understanding their pathogenesis to illuminate intervention strategies. Curr. Opin. Neurol. 25, 50-56.

Glascock, J.J., Osman, E.Y., Coady, T.H., Rose, F.F., Shababi, M., and Lorson, C.L. (2011). Delivery of therapeutic agents through intracerebroventricular (ICV) and intravenous (IV) injection in mice. J. Vis. Exp. JoVE. 
Gleich, O., and Wilson, S. (1993). The diameters of guinea pig auditory nerve fibres: Distribution and correlation with spontaneous rate. Hear. Res. 71, 69-79.

Goldberg, J.M., and Brown, P.B. (1969). Response of binaural neurons of dog superior olivary complex to dichotic tonal stimuli: some physiological mechanisms of sound localization. J. Neurophysiol. 32, 613-636.

Goßler, C., Bierbrauer, C., Moser, R., Kunzer, M., Holc, K., Pletschen, W., Köhler, K., Wagner, J., Schwaerzle, M., Ruther, P., et al. (2014). GaN-based micro-LED arrays on flexible substrates for optical cochlear implants. J. Phys. Appl. Phys. 47, 205401.

Govorunova, E.G., Spudich, E.N., Lane, C.E., Sineshchekov, O.A., and Spudich, J.L. (2011). New Channelrhodopsin with a Red-Shifted Spectrum and Rapid Kinetics from Mesostigma viride. MBio 2.

Govorunova, E.G., Sineshchekov, O.A., Janz, R., Liu, X., and Spudich, J.L. (2015). Natural light-gated anion channels: A family of microbial rhodopsins for advanced optogenetics. Science 349, 647-650.

Gradinaru, V., Zhang, F., Ramakrishnan, C., Mattis, J., Prakash, R., Diester, I., Goshen, I., Thompson, K.R., and Deisseroth, K. (2010). Molecular and Cellular Approaches for Diversifying and Extending Optogenetics. Cell 141, 154-165.

Gray, S.J., Choi, V.W., Asokan, A., Haberman, R.A., McCown, T.J., and Samulski, R.J. (2011). Production of Recombinant Adeno-Associated Viral Vectors and Use in In Vitro and In Vivo Administration. In Current Protocols in Neuroscience, J.N. Crawley, C.R. Gerfen, M.A. Rogawski, D.R. Sibley, P. Skolnick, and S. Wray, eds. (Hoboken, NJ, USA: John Wiley \& Sons, Inc.), p.

Graydon, C.W., Cho, S., Li, G.-L., Kachar, B., and Gersdorff, H. von (2011). Sharp Ca2+ Nanodomains beneath the Ribbon Promote Highly Synchronous Multivesicular Release at Hair Cell Synapses. J. Neurosci. 31, 16637-16650.

Greenwood, D.D. (1996). Comparing octaves, frequency ranges, and cochlear-map curvature across species. Hear. Res. 94, 157-162.

Grieger, J.C., Choi, V.W., and Samulski, R.J. (2006). Production and characterization of adenoassociated viral vectors. Nat. Protoc. 1, 1412-1428.

Grothe, B., Pecka, M., and McAlpine, D. (2010). Mechanisms of Sound Localization in Mammals. Physiol. Rev. 90, 983-1012.

Grubb, M.S., and Burrone, J. (2010). Channelrhodopsin-2 Localised to the Axon Initial Segment. PLoS ONE 5, e13761.

Gueta, R., Barlam, D., Shneck, R.Z., and Rousso, I. (2006). Measurement of the mechanical properties of isolated tectorial membrane using atomic force microscopy. Proc. Natl. Acad. Sci. 103, 14790-14795.

Guinan, J.J., Jr (2010). Cochlear efferent innervation and function. Curr. Opin. Otolaryngol. Head Neck Surg. 18, 447-453.

Gunaydin, L.A., Yizhar, O., Berndt, A., Sohal, V.S., Deisseroth, K., and Hegemann, P. (2010). Ultrafast optogenetic control. Nat. Neurosci. 13, 387-392.

Guo, W., Hight, A.E., Chen, J.X., Klapoetke, N.C., Hancock, K.E., Shinn-Cunningham, B.G., Boyden, E.S., Lee, D.J., and Polley, D.B. (2015). Hearing the light: neural and perceptual encoding of optogenetic stimulation in the central auditory pathway. Sci. Rep. 5.

György, B., Sage, C., Indzhykulian, A.A., Scheffer, D.I., Brisson, A.R., Tan, S., Wu, X., Volak, A., Mu, D., Tamvakologos, P.I., et al. (2017). Rescue of Hearing by Gene Delivery to Inner-Ear Hair Cells Using Exosome-Associated AAV. Mol. Ther. 25, 379-391. 
Ham, W.T., Mueller, H.A., and Sliney, D.H. (1976). Retinal sensitivity to damage from short wavelength light. Nature 260, 153-155.

Han, X., Qian, X., Bernstein, J.G., Zhou, H.-H., Franzesi, G.T., Stern, P., Bronson, R.T., Graybiel, A.M., Desimone, R., and Boyden, E.S. (2009). Millisecond-timescale optical control of neural dynamics in the nonhuman primate brain. Neuron 62, 191-198.

Haupts, U., Tittor, J., and Oesterhelt, D. (1999). Closing in on bacteriorhodopsin: progress in understanding the molecule. Annu. Rev. Biophys. Biomol. Struct. 28, 367-399.

Heffner, H.E., and Heffner, R.S. (2008). High-Frequency Hearing. In The Senses: A Comprehensive Reference, (New York: Academic Press), pp. 55-60.

Heil, P., and Peterson, A.J. (2015). Basic response properties of auditory nerve fibers: a review. Cell Tissue Res. 361, 129-158.

Helms, J., Müller, J., Schön, F., Moser, L., Arnold, W., Janssen, T., Ramsden, R., von Ilberg, C., Kiefer, J., Pfennigdorf, T., et al. (1997). Evaluation of performance with the COMBI40 cochlear implant in adults: a multicentric clinical study. ORL J. Oto-Rhino-Laryngol. Its Relat. Spec. 59, 23-35.

Henry, W.R., and Mulroy, M.J. (1995). Afferent synaptic changes in auditory hair cells during noiseinduced temporary threshold shift. Hear. Res. 84, 81-90.

Hernandez, V.H., Gehrt, A., Reuter, K., Jing, Z., Jeschke, M., Mendoza Schulz, A., Hoch, G., Bartels, M., Vogt, G., Garnham, C.W., et al. (2014a). Optogenetic stimulation of the auditory pathway. J. Clin. Invest. $124,1114-1129$.

Hernandez, V.H., Gehrt, A., Jing, Z., Hoch, G., Jeschke, M., Strenzke, N., and Moser, T. (2014b). Optogenetic Stimulation of the Auditory Nerve. J. Vis. Exp. e52069.

Hight, A.E., Kozin, E.D., Darrow, K., Lehmann, A., Boyden, E., Brown, M.C., and Lee, D.J. (2015). Superior temporal resolution of Chronos versus channelrhodopsin-2 in an optogenetic model of the auditory brainstem implant. Hear. Res. 322, 235-241.

Hille, B. (2001). In Ionic Channels of Excitable Membranes, (Sunderland, Mass: Sinauer Associates), pp. $441-470$.

Hillery, C.M., and Narins, P.M. (1987). Frequency and time domain comparison of low-frequency auditory fiber responses in two anuran amphibians. Hear. Res. 25, 233-248.

Hippenmeyer, S., Vrieseling, E., Sigrist, M., Portmann, T., Laengle, C., Ladle, D.R., and Arber, S. (2005). A developmental switch in the response of DRG neurons to ETS transcription factor signaling. PLoS Biol. 3, e159.

Hirose, K., and Liberman, M.C. (2003). Lateral Wall Histopathology and Endocochlear Potential in the Noise-Damaged Mouse Cochlea. JARO J. Assoc. Res. Otolaryngol. 4, 339-352.

Hofherr, A. (2005). Selective Golgi export of Kir2.1 controls the stoichiometry of functional Kir2.x channel heteromers. J. Cell Sci. 118, 1935-1943.

Hofherr, A., Fakler, B., and Klöcker, N. (2005). Selective Golgi export of Kir2.1 controls the stoichiometry of functional Kir2.x channel heteromers. J. Cell Sci. 118, 1935-1943.

Hossain, W.A., Antic, S.D., Yang, Y., Rasband, M.N., and Morest, D.K. (2005). Where is the spike generator of the cochlear nerve? Voltage-gated sodium channels in the mouse cochlea. J. Neurosci. 25, $6857-6868$. 
Hu, H., Gan, J., and Jonas, P. (2014). Interneurons. Fast-spiking, parvalbumin ${ }^{+}$GABAergic interneurons: from cellular design to microcircuit function. Science 345, 1255263.

Hu, N., Abbas, P.J., Miller, C.A., Robinson, B.K., Nourski, K.V., Jeng, F.-C., Abkes, B.A., and Nichols, J.M. (2003). Auditory response to intracochlear electric stimuli following furosemide treatment. Hear. Res. 185, 77-89.

Hudspeth, A.J. (2014). Integrating the active process of hair cells with cochlear function. Nat. Rev. Neurosci. 15, 600 .

Huet, A., Desmadryl, G., Justal, T., Nouvian, R., Puel, J.-L., and Bourien, J. (2018). The Interplay Between Spike-Time and Spike-Rate Modes in the Auditory Nerve Encodes Tone-In-Noise Threshold. J. Neurosci. 38, 5727-5738.

Izumikawa, M., Minoda, R., Kawamoto, K., Abrashkin, K.A., Swiderski, D.L., Dolan, D.F., Brough, D.E., and Raphael, Y. (2005). Auditory hair cell replacement and hearing improvement by Atoh1 gene therapy in deaf mammals. Nat. Med. 11, 271-276.

Izzo, A.D., Richter, C.-P., Jansen, E.D., and Walsh, J.T., Jr (2006). Laser stimulation of the auditory nerve. Lasers Surg. Med. 38, 745-753.

Izzo, A.D., Suh, E., Pathria, J., Walsh, J.T., Whitlon, D.S., and Richter, C.-P. (2007). Selectivity of neural stimulation in the auditory system: a comparison of optic and electric stimuli. J. Biomed. Opt. 12, 021008 .

Izzo, A.D., Walsh, J.T., Jr, Ralph, H., Webb, J., Bendett, M., Wells, J., and Richter, C.-P. (2008). Laser stimulation of auditory neurons: effect of shorter pulse duration and penetration depth. Biophys. J. 94 , 3159-3166.

Jackman, S.L., Choi, S.-Y., Thoreson, W.B., Rabl, K., Bartoletti, T.M., and Kramer, R.H. (2009). Role of the synaptic ribbon in transmitting the cone light response. Nat. Neurosci. 12, 303.

Jacques, S.L. (2013). Optical properties of biological tissues: a review. Phys. Med. Biol. 58, R37-R61.

Javel, E. (1990). Acoustic and Electrical Encoding of Temporal Information. In Cochlear Implants: Models of the Electrically Stimulated Ear, J.M. Miller, and F.A. Spelman, eds. (Springer Verlag, New York, Inc), p.

Jean, P., Lopez de la Morena, D., Michanski, S., Jaime Tobón, L.M., Chakrabarti, R., Picher, M.M., Neef, J., Jung, S., Gültas, M., Maxeiner, S., et al. (2018). The synaptic ribbon is critical for sound encoding at high rates and with temporal precision. ELife 7.

Jeschke, M., and Moser, T. (2015). Considering optogenetic stimulation for cochlear implants. Hear. Res. 322, 224-234.

Johnson, D.H. (1980). The relationship between spike rate and synchrony in responses of auditory-nerve fibers to single tones. J. Acoust. Soc. Am. 68, 1115-1122.

Johnson, C.E., Jilla, A.M., Danhauer, J.L., Sullivan, J.C., and Sanchez, K.R. (2018). Benefits from, Satisfaction with, and Self-Efficacy for Advanced Digital Hearing Aids in Users with Mild Sensorineural Hearing Loss. Semin. Hear. 39, 158-171.

Joris, P.X., Schreiner, C.E., and Rees, A. (2004). Neural Processing of Amplitude-Modulated Sounds. Physiol Rev 84, 541-577.

Jung, S., Maritzen, T., Wichmann, C., Jing, Z., Neef, A., Revelo, N.H., Al-Moyed, H., Meese, S., Wojcik, S.M., Panou, I., et al. (2015). Disruption of adaptor protein $2 \mu$ (AP-2 $\mu$ ) in cochlear hair cells impairs vesicle reloading of synaptic release sites and hearing. EMBO J. 34, 2686-2702. 
Kallweit, N., Baumhoff, P., Krueger, A., Tinne, N., Kral, A., Ripken, T., and Maier, H. (2016). Optoacoustic effect is responsible for laser-induced cochlear responses. Sci. Rep. 6.

Kampasi, K., English, D.F., Seymour, J., Stark, E., McKenzie, S., Vöröslakos, M., Buzsáki, G., Wise, K.D., and Yoon, E. (2018). Dual color optogenetic control of neural populations using low-noise, multishank optoelectrodes. Microsyst. Nanoeng. 4, 10.

Kandel, E.R., Schwartz, J.H., and Jessell, T. (2012). Principles of neural science (New York: McGrawHill Medical).

Kane, K.L., Longo-Guess, C.M., Gagnon, L.H., Ding, D., Salvi, R.J., and Johnson, K.R. (2012). Genetic background effects on age-related hearing loss associated with Cdh23 variants in mice. Hear. Res. 283, $80-88$.

Kato, H.E., Zhang, F., Yizhar, O., Ramakrishnan, C., Nishizawa, T., Hirata, K., Ito, J., Aita, Y., Tsukazaki, T., Hayashi, S., et al. (2012). Crystal structure of the channelrhodopsin light-gated cation channel. Nature 482, 369-374.

Khan, A.M., Handzel, O., Burgess, B.J., Damian, D., Eddington, D.K., and Nadol, J.B. (2005). Is word recognition correlated with the number of surviving spiral ganglion cells and electrode insertion depth in human subjects with cochlear implants? The Laryngoscope 115, 672-677.

Khimich, D., Nouvian, R., Pujol, R., tom Dieck, S., Egner, A., Gundelfinger, E.D., and Moser, T. (2005). Hair cell synaptic ribbons are essential for synchronous auditory signalling. Nature 434, 889-894.

Kiang, N.Y.S., Watanabe, T., Thomas, E.C., and Clark, L.F. (1965). Discharge Patterns of Single Fibers in the Cat's Auditory Nerve (Cambridge, Massachusetts: MIT Press).

Kiang, N.Y.S., Liberman, M.C., and Levine, R.A. (1976). Auditory-Nerve Activity in Cats Exposed to Ototoxic Drugs and High-Intensity Sounds. Ann. Otol. Rhinol. Laryngol. 85, 752-768.

Kiefer, J., Hohl, S., Stürzebecher, E., Pfennigdorff, T., and Gstöettner, W. (2001). Comparison of speech recognition with different speech coding strategies (SPEAK, CIS, and ACE) and their relationship to telemetric measures of compound action potentials in the nucleus CI $24 \mathrm{M}$ cochlear implant system. Audiol. Off. Organ Int. Soc. Audiol. 40, 32-42.

Kim, C.K., Adhikari, A., and Deisseroth, K. (2017). Integration of optogenetics with complementary methodologies in systems neuroscience. Nat. Rev. Neurosci. 18, 222-235.

Klapoetke, N.C., Murata, Y., Kim, S.S., Pulver, S.R., Birdsey-Benson, A., Cho, Y.K., Morimoto, T.K., Chuong, A.S., Carpenter, E.J., Tian, Z., et al. (2014). Independent optical excitation of distinct neural populations. Nat. Methods 11,338-346.

Klapper, S.D., Swiersy, A., Bamberg, E., and Busskamp, V. (2016). Biophysical Properties of Optogenetic Tools and Their Application for Vision Restoration Approaches. Front. Syst. Neurosci. 10.

Kleinlogel, S., Feldbauer, K., Dempski, R.E., Fotis, H., Wood, P.G., Bamann, C., and Bamberg, E. (2011). Ultra light-sensitive and fast neuronal activation with the $\mathrm{Ca}^{2}+$-permeable channelrhodopsin CatCh. Nat. Neurosci. 14, 513-518.

Koch, D.B., Downing, M., Osberger, M.J., and Litvak, L. (2007). Using current steering to increase spectral resolution in CII and HiRes 90K users. Ear Hear. 28, 38S-41S.

Köppl, C. (1997). Phase locking to high frequencies in the auditory nerve and cochlear nucleus magnocellularis of the barn owl, Tyto alba. J. Neurosci. 17, 3312.

Kral, A., Hartmann, R., Mortazavi, D., and Klinke, R. (1998). Spatial resolution of cochlear implants: the electrical field and excitation of auditory afferents. Hear. Res. 121, 11-28. 
Kuhne, J., Eisenhauer, K., Ritter, E., Hegemann, P., Gerwert, K., and Bartl, F. (2015). Early formation of the ion-conducting pore in channelrhodopsin-2. Angew. Chem. Int. Ed Engl. 54, 4953-4957.

Kujawa, S.G., and Liberman, M.C. (2006). Acceleration of age-related hearing loss by early noise exposure: evidence of a misspent youth. J. Neurosci. 26, 2115.

Kujawa, S.G., and Liberman, M.C. (2009). Adding insult to injury: cochlear nerve degeneration after "temporary" noise-induced hearing loss. J. Neurosci. 29, 14077-14085.

Kujawa, S.G., and Liberman, M.C. (2015). Synaptopathy in the noise-exposed and aging cochlea: Primary neural degeneration in acquired sensorineural hearing loss. Hear. Res.

Labadie, R.F., Noble, J.H., Hedley-Williams, A.J., Sunderhaus, L.W., Dawant, B.M., and Gifford, R.H. (2016). Results of Postoperative, CT-based, Electrode Deactivation on Hearing in Prelingually Deafened Adult Cochlear Implant Recipients. Otol. Neurotol. Off. Publ. Am. Otol. Soc. Am. Neurotol. Soc. Eur. Acad. Otol. Neurotol. 37, 137-145.

Lagnado, L., and Schmitz, F. (2015). Ribbon Synapses and Visual Processing in the Retina. Annu. Rev. Vis. Sci. 1, 235-262.

Lalwani, A.K., Walsh, B.J., Reilly, P.G., Muzyczka, N., and Mhatre, A.N. (1996). Development of in vivo gene therapy for hearing disorders: introduction of adeno-associated virus into the cochlea of the guinea pig. Gene Ther. 3, 588-592.

Landsberger, D.M., and Srinivasan, A.G. (2009). Virtual channel discrimination is improved by current focusing in cochlear implant recipients. Hear. Res. 254, 34-41.

Leake, P.A., and Rebscher, S.J. (2004). Anatomical considerations and long-term effects of electri cal stimulation. In Cochlear Implants: Auditory Prostheses and Electric Hearing, F.-G. Zeng, A.N. Popper, and R.R. Fay, eds. (Springer Science \& Business Media), p.

Ledentsov, N.N., Shchukin, V.A., Shernyakov, Y.M., Kulagina, M.M., Payusov, A.S., Gordeev, N.Y., Maximov, M.V., Zhukov, A.E., Denneulin, T., and Cherkashin, N. (2018). Room-temperature yelloworange (In,Ga,Al)P-GaP laser diodes grown on (n11) GaAs substrates. Opt. Express 26, 13985-13994.

Lenarz, T. (2018). Cochlear implant - state of the art. GMS Curr. Top. Otorhinolaryngol. Head Neck Surg. 16, Doc04.

Lenarz, M., Sönmez, H., Joseph, G., Büchner, A., and Lenarz, T. (2012). Long-term performance of cochlear implants in postlingually deafened adults. Otolaryngol.--Head Neck Surg. Off. J. Am. Acad. Otolaryngol.-Head Neck Surg. 147, 112-118.

Lewis, T.L., Mao, T., Svoboda, K., and Arnold, D.B. (2009). Myosin-dependent targeting of transmembrane proteins to neuronal dendrites. Nat. Neurosci. 12, 568-576.

Liao, L.-D., Chang, Y.-J., Lai, H.-Y., Lin, C.-T., Lin, Z.-M., Tsang, S., and Chen, Y.-Y. (2012). A Novel Light-Addressable Multi-Electrode Array Chip for Neural Signal Recording Based on VCSEL Diode Arrays. J. Neurosci. Neuroengineering 1, 4-12.

Liberman, M. (1982a). Single-neuron labeling in the cat auditory nerve. Science 216, 1239-1241.

Liberman, M.C. (1978). Auditory-nerve response from cats raised in a low-noise chamber. J. Acoust. Soc. Am. 63, 442-455.

Liberman, M.C. (1980). Morphological differences among radial afferent fibers in the cat cochlea: an electron-microscopic study of serial sections. Hear. Res. 3, 45-63. 
Liberman, M.C. (1982b). The cochlear frequency map for the cat: labeling auditory-nerve fibers of known characteristic frequency. J. Acoust. Soc. Am. 72, 1441-1449.

Liberman, M.C. (2017). Noise-induced and age-related hearing loss: new perspectives and potential therapies. F1000Research 6.

Liberman, M.C., and Oliver, M.E. (1984). Morphometry of intracellularly labeled neurons of the auditory nerve: Correlations with functional properties. J. Comp. Neurol. 223, 163-176.

Liberman, L.D., Wang, H., and Liberman, M.C. (2011). Opposing Gradients of Ribbon Size and AMPA Receptor Expression Underlie Sensitivity Differences among Cochlear-Nerve/Hair-Cell Synapses. J. Neurosci. 31, 801-808.

Liberman, M.C., Dodds, L.W., and Pierce, S. (1990). Afferent and efferent innervation of the cat cochlea: quantitative analysis with light and electron microscopy. J. Comp. Neurol. 301, 443-460.

Lin, T., and Guinan Jr, J.J. (2000). Auditory-nerve-fiber responses to high-level clicks: Interference patterns indicate that excitation is due to the combination of multiple drives. J. Acoust. Soc. Am. 107, 2615.

Lin, T., and Guinan Jr, J.J. (2004). Time-frequency analysis of auditory-nerve-fiber and basilarmembrane click responses reveal glide irregularities and non-characteristic-frequency skirts. J. Acoust. Soc. Am. 116, 405-416.

Lin, J.Y., Lin, M.Z., Steinbach, P., and Tsien, R.Y. (2009). Characterization of engineered channelrhodopsin variants with improved properties and kinetics. Biophys. J. 96, 1803-1814.

Lin, J.Y., Knutsen, P.M., Muller, A., Kleinfeld, D., and Tsien, R.Y. (2013). ReaChR: A red-shifted variant of channelrhodopsin enables deep transcranial optogenetic excitation. Nat. Neurosci. 16, 14991508 .

Litvak, L., Delgutte, B., and Eddington, D. (2001). Auditory nerve fiber responses to electric stimulation: Modulated and unmodulated pulse trains. J. Acoust. Soc. Am. 110, 368-379.

Liu, C., Glowatzki, E., and Fuchs, P.A. (2015). Unmyelinated type II afferent neurons report cochlear damage. Proc. Natl. Acad. Sci. U. S. A. 112, 14723-14727.

Lorenz, C., Pusch, M., and Jentsch, T.J. (1996). Heteromultimeric CLC chloride channels with novel properties. Proc. Natl. Acad. Sci. U. S. A. 93, 13362-13366.

Lórenz-Fonfría, V.A., Bamann, C., Resler, T., Schlesinger, R., Bamberg, E., and Heberle, J. (2015). Temporal evolution of helix hydration in a light-gated ion channel correlates with ion conductance. Proc. Natl. Acad. Sci. 112, E5796-E5804.

Lu, T.-C., Jun-Rong, C., Shih-Wei, C., Hao-Chung, K., Chien-Cheng, K., Cheng-Chung, L., and ShingChung, W. (2009). Development of GaN-Based Vertical-Cavity Surface-Emitting Lasers. IEEE J. Sel. Top. Quantum Electron. 15, 850-860.

Luecke, H. (2001). Crystal Structure of Sensory Rhodopsin II at 2.4 Angstroms: Insights into Color Tuning and Transducer Interaction. Science 293, 1499-1503.

Lysakowski, A., Gaboyard-Niay, S., Calin-Jageman, I., Chatlani, S., Price, S.D., and Eatock, R.A. (2011). Molecular Microdomains in a Sensory Terminal, the Vestibular Calyx Ending. J. Neurosci. 31, 10101.

Ma, D., Zerangue, N., Lin, Y.-F., Collins, A., Yu, M., Jan, Y.N., and Jan, L.Y. (2001). Role of ER Export Signals in Controlling Surface Potassium Channel Numbers. Science 291, 316-319. 
Maddalena, A., Tereshchenko, J., Bähr, M., and Kügler, S. (2013). Adeno-associated Virus-mediated, Mifepristone-regulated Transgene Expression in the Brain. Mol. Ther. Acids 2, e106.

Madisen, L., Zwingman, T.A., Sunkin, S.M., Oh, S.W., Zariwala, H.A., Gu, H., Ng, L.L., Palmiter, R.D., Hawrylycz, M.J., Jones, A.R., et al. (2010). A robust and high-throughput Cre reporting and characterization system for the whole mouse brain. Nat. Neurosci. 13, 133-140.

Mager, T., Lopez de la Morena, D., Senn, V., Schlotte, J., D Errico, A., Feldbauer, K., Wrobel, C., Jung, S., Bodensiek, K., Rankovic, V., et al. (2018). High frequency neural spiking and auditory signaling by ultrafast red-shifted optogenetics. Nat. Commun. 9, 1750.

Magnusson, L. (2011). Comparison of the fine structure processing (FSP) strategy and the CIS strategy used in the MED-EL cochlear implant system: speech intelligibility and music sound quality. Int. J. Audiol. 50, 279-287.

Magupalli, V.G., Schwarz, K., Alpadi, K., Natarajan, S., Seigel, G.M., and Schmitz, F. (2008). Multiple RIBEYE-RIBEYE interactions create a dynamic scaffold for the formation of synaptic ribbons. J. Neurosci. Off. J. Soc. Neurosci. 28, 7954-7967.

Mann, Z.F., and Kelley, M.W. (2011). Development of tonotopy in the auditory periphery. Hear. Res. $276,2-15$.

Mardinly, A.R., Oldenburg, I.A., Pégard, N.C., Sridharan, S., Lyall, E.H., Chesnov, K., Brohawn, S.G., Waller, L., and Adesnik, H. (2018). Precise multimodal optical control of neural ensemble activity. Nat. Neurosci. 21, 881-893.

Marek, V., Mélik-Parsadaniantz, S., Villette, T., Montoya, F., Baudouin, C., Brignole-Baudouin, F., and Denoyer, A. (2018). Blue light phototoxicity toward human corneal and conjunctival epithelial cells in basal and hyperosmolar conditions. Free Radic. Biol. Med. 126, 27-40.

Matsuno-Yagi, A., and Mukohata, Y. (1977). Two possible roles of bacteriorhodopsin; a comparative study of strains of Halobacterium halobium differing in pigmentation. Biochem. Biophys. Res. Commun. $78,237-243$.

Matthews, G., and Fuchs, P. (2010). The diverse roles of ribbon synapses in sensory neurotransmission. Nat. Rev. Neurosci. 11, 812-822.

Mattis, J., Tye, K.M., Ferenczi, E.A., Ramakrishnan, C., O’Shea, D.J., Prakash, R., Gunaydin, L.A., Hyun, M., Fenno, L.E., Gradinaru, V., et al. (2011). Principles for applying optogenetic tools derived from direct comparative analysis of microbial opsins. Nat. Methods 9, 159-172.

Maxeiner, S., Luo, F., Tan, A., Schmitz, F., and Südhof, T.C. (2016). How to make a synaptic ribbon: RIBEYE deletion abolishes ribbons in retinal synapses and disrupts neurotransmitter release. EMBO J. $35,1098-1114$.

Meyer, A.C., and Moser, T. (2010). Structure and function of cochlear afferent innervation. Curr. Opin. Otolaryngol. Head Neck Surg. 18, 441-446.

Middlebrooks, J.C., and Snyder, R.L. (2007). Auditory prosthesis with a penetrating nerve array. J. Assoc. Res. Otolaryngol. JARO 8, 258-279.

Miller, C.A., Abbas, P.J., Robinson, B.K., Rubinstein, J.T., and Matsuoka, A.J. (1999). Electrically evoked single- $\phi$ ber action potentials from cat: responses to monopolar, monophasic stimulation. Hear. Res. 22.

Miller, C.A., Abbas, P.J., and Robinson, B.K. (2001). Response properties of the refractory auditory nerve fiber. JARO-J. Assoc. Res. Otolaryngol. 2, 216-232. 
Miller, C.A., Abbas, P.J., Robinson, B.K., Nourski, K.V., Zhang, F., and Jeng, F.-C. (2006). Electrical Excitation of the Acoustically Sensitive Auditory Nerve: Single-Fiber Responses to Electric Pulse Trains. J. Assoc. Res. Otolaryngol. 7, 195-210.

Miller, C.A., Hu, N., Zhang, F., Robinson, B.K., and Abbas, P.J. (2008). Changes Across Time in the Temporal Responses of Auditory Nerve Fibers Stimulated by Electric Pulse Trains. JARO J. Assoc. Res. Otolaryngol. 9, 122-137.

Min, K.S., Jun, S.B., Lim, Y.S., Park, S.-I., and Kim, S.J. (2013). Modiolus-Hugging Intracochlear Electrode Array with Shape Memory Alloy. Comput. Math. Methods Med. 2013, 1-9.

Miura, M., Sando, I., Hirsch, B.E., and Orita, Y. (2002). Analysis of spiral ganglion cell populations in children with normal and pathological ears. Ann. Otol. Rhinol. Laryngol. 111, 1059-1065.

Miyashita, T., Shao, Y.R., Chung, J., Pourzia, O., and Feldman, D.E. (2013). Long-term channelrhodopsin-2 (ChR2) expression can induce abnormal axonal morphology and targeting in cerebral cortex. Front. Neural Circuits 7.

Moser, T. (2015a). Optogenetic stimulation of the auditory pathway for research and future prosthetics. Curr. Opin. Neurobiol. 34, 29-36.

Moser, T. (2015b). Gene therapy for deafness: How close are we? Sci. Transl. Med. 7, 295fs28-295fs28.

Moser, T., and Starr, A. (2016). Auditory neuropathy — neural and synaptic mechanisms. Nat. Rev. Neurol. 12, 135-149.

Moser, T., and Vogl, C. (2016). New insights into cochlear sound encoding. F1000Research 5, 2081.

Mueller, C., and Flotte, T.R. (2008). Clinical gene therapy using recombinant adeno-associated virus vectors. Gene Ther. 15, 858-863.

Müller, U., and Barr-Gillespie, P.G. (2015). New treatment options for hearing loss. Nat. Rev. Drug Discov. 14, 346-365.

Müller, M., Bamann, C., Bamberg, E., and Kühlbrandt, W. (2015). Light-induced helix movements in channelrhodopsin-2. J. Mol. Biol. 427, 341-349.

Nagel, G., Möckel, B., Büldt, G., and Bamberg, E. (1995). Functional expression of bacteriorhodopsin in oocytes allows direct measurement of voltage dependence of light induced H+ pumping. FEBS Lett. 377, 263-266.

Nagel, G., Ollig, D., Fuhrmann, M., Kateriya, S., Musti, A.M., Bamberg, E., and Hegemann, P. (2002). Channelrhodopsin-1: a light-gated proton channel in green algae. Science 296, 2395-2398.

Nagel, G., Szellas, T., Huhn, W., Kateriya, S., Adeishvili, N., Berthold, P., Ollig, D., Hegemann, P., and Bamberg, E. (2003). Channelrhodopsin-2, a directly light-gated cation-selective membrane channel. Proc. Natl. Acad. Sci. 100, 13940-13945.

Nagel, G., Szellas, T., Kateriya, S., Adeishvili, N., Hegemann, P., and Bamberg, E. (2005a). Channelrhodopsins: directly light-gated cation channels. Biochem. Soc. Trans. 33, 863-866.

Nagel, G., Brauner, M., Liewald, J.F., Adeishvili, N., Bamberg, E., and Gottschalk, A. (2005b). Light activation of channelrhodopsin-2 in excitable cells of Caenorhabditis elegans triggers rapid behavioral responses. Curr. Biol. CB 15, 2279-2284.

Nakanishi, T., Kanada, S., Murakami, M., Ihara, K., and Kouyama, T. (2013). Large Deformation of Helix F during the Photoreaction Cycle of Pharaonis Halorhodopsin in Complex with Azide. Biophys. J. 104, 377-385. 
Naso, M.F., Tomkowicz, B., Perry, W.L., and Strohl, W.R. (2017). Adeno-Associated Virus (AAV) as a Vector for Gene Therapy. Biodrugs 31, 317-334.

Nathwani, A.C., Rosales, C., McIntosh, J., Rastegarlari, G., Nathwani, D., Raj, D., Nawathe, S., Waddington, S.N., Bronson, R., Jackson, S., et al. (2011). Long-term Safety and Efficacy Following Systemic Administration of a Self-complementary AAV Vector Encoding Human FIX Pseudotyped With Serotype 5 and 8 Capsid Proteins. Mol. Ther. 19, 876-885.

Nie, K., Stickney, G., and Zeng, F.-G. (2005). Encoding frequency modulation to improve cochlear implant performance in noise. IEEE Trans. Biomed. Eng. 52, 64-73.

Nikolic, K., Grossman, N., Grubb, M.S., Burrone, J., Toumazou, C., and Degenaar, P. (2009). Photocycles of Channelrhodopsin-2. Photochem. Photobiol. 85, 400-411.

Nogueira, W., Litvak, L.M., Saoji, A.A., and Büchner, A. (2015). Design and Evaluation of a Cochlear Implant Strategy Based on a "Phantom" Channel. PLOS ONE 10, e0120148.

Oda, K., Vierock, J., Oishi, S., Rodriguez-Rozada, S., Taniguchi, R., Yamashita, K., Wiegert, J.S., Nishizawa, T., Hegemann, P., and Nureki, O. (2018). Crystal structure of the red light-activated channelrhodopsin Chrimson. Nat. Commun. 9, 3949.

Oesterhelt, D., and Stoeckenius, W. (1971). Rhodopsin-like Protein from the Purple Membrane of Halobacterium halobium. Nature. New Biol. 233, 149-152.

Ohlemiller, K.K., and Echteler, S.M. (1990). Functional correlates of characteristic frequency in single cochlear nerve fibers of the Mongolian gerbil. J. Comp. Physiol. [A] 167, 329-338.

Ohlemiller, K.K., Echteler, S.M., and Siegel, J.H. (1991). Factors that influence rate-versus-intensity relations in single cochlear nerve fibers of the gerbil. J. Acoust. Soc. Am. 90, 274-287.

Ohlemiller, K.K., Jones, S.M., and Johnson, K.R. (2016). Application of Mouse Models to Research in Hearing and Balance. J. Assoc. Res. Otolaryngol. 17, 493-523.

Ohn, T.L., Rutherford, M.A., Jing, Z.Z., Jung, S.Y., Duque-Afonso, C.J., Hoch, G., Picher, M.M., Scharinger, A., Strenzke, N., and Moser, T. (2016). Hair cells use active zones with different voltage dependence of $\mathrm{Ca} 2+$ influx to decompose sounds into complementary neural codes. Proc. Natl. Acad. Sci. U. S. A. 113, E4716-E4725.

Palmer, A.R., and Russell, I.J. (1986). Phase-locking in the cochlear nerve of the guinea-pig and its relation to the receptor potential of inner hair-cells. Hear. Res. 24, 1-15.

Pan, B., Askew, C., Galvin, A., Heman-Ackah, S., Asai, Y., Indzhykulian, A.A., Jodelka, F.M., Hastings, M.L., Lentz, J.J., Vandenberghe, L.H., et al. (2017). Gene therapy restores auditory and vestibular function in a mouse model of Usher syndrome type 1c. Nat. Biotechnol. 35, 264-272.

Pangrsic, T., Reisinger, E., and Moser, T. (2012). Otoferlin: a multi-C-2 domain protein essential for hearing. Trends Neurosci. 35, 671-680.

Pfeiffer, R.R., and Kim, D.O. (1972). Response Patterns of Single Cochlear Nerve Fibers to Click Stimuli: Descriptions for Cat. J. Acoust. Soc. Am. 52, 1669-1677.

Picher, M.M., Gehrt, A., Meese, S., Ivanovic, A., Predoehl, F., Jung, S., Schrauwen, I., Dragonetti, A.G., Colombo, R., Camp, G.V., et al. (2017). Ca2+-binding protein 2 inhibits Ca2+-channel inactivation in mouse inner hair cells. Proc. Natl. Acad. Sci. 114, E1717-E1726.

Pinyon, J.L., Tadros, S.F., Froud, K.E., Y Wong, A.C., Tompson, I.T., Crawford, E.N., Ko, M., Morris, R., Klugmann, M., and Housley, G.D. (2014). Close-field electroporation gene delivery using the cochlear implant electrode array enhances the bionic ear. Sci. Transl. Med. 6, 233ra54-233ra54. 
Platzer, J., Engel, J., Schrott-Fischer, A., Stephan, K., Bova, S., Chen, H., Zheng, H., and Striessnig, J. (2000). Congenital deafness and sinoatrial node dysfunction in mice lacking class D L-type Ca2+ channels. Cell 102, 89-97.

Prakash, R., Yizhar, O., Grewe, B., Ramakrishnan, C., Wang, N., Goshen, I., Packer, A.M., Peterka, D.S., Yuste, R., Schnitzer, M.J., et al. (2012). Two-photon optogenetic toolbox for fast inhibition, excitation and bistable modulation. Nat. Methods 9, 1171-1179.

Puel, J.L., Pujol, R., Ladrech, S., and Eybalin, M. (1991). Alpha-amino-3-hydroxy-5-methyl-4-isoxazole propionic acid electrophysiological and neurotoxic effects in the guinea-pig cochlea. Neuroscience 45 , 63-72.

Purves, D., Augustine, G.J., Fitzpatrick, D., Katz, L.C., LaMantia, A.S., McNamara, J.O., and Williams, S.M. (2004). Neuroscience (Sunderland, Mass: Sinauer Associates, Publishers).

Rajasethupathy, P., Sankaran, S., Marshel, J.H., Kim, C.K., Ferenczi, E., Lee, S.Y., Berndt, A., Ramakrishnan, C., Jaffe, A., Lo, M., et al. (2015). Projections from neocortex mediate top-down control of memory retrieval. Nature 526, 653-659.

Ramachandran, P.S., Lee, V., Wei, Z., Song, J.Y., Casal, G., Cronin, T., Willett, K., Huckfeldt, R., Morgan, J.I.W., Aleman, T.S., et al. (2017). Evaluation of Dose and Safety of AAV7m8 and AAV8BP2 in the Non-Human Primate Retina. Hum. Gene Ther. 28, 154-167.

Rattay, F., Potrusil, T., Wenger, C., Wise, A.K., Glueckert, R., and Schrott-Fischer, A. (2013). Impact of Morphometry, Myelinization and Synaptic Current Strength on Spike Conduction in Human and Cat Spiral Ganglion Neurons. PLoS ONE 8, e79256.

Reisinger, E., Bresee, C., Neef, J., Nair, R., Reuter, K., Bulankina, A., Nouvian, R., Koch, M., Bückers, J., Kastrup, L., et al. (2011). Probing the functional equivalence of otoferlin and synaptotagmin 1 in exocytosis. J. Neurosci. Off. J. Soc. Neurosci. 31, 4886-4895.

Rettenmaier, A., Lenarz, T., and Reuter, G. (2014). Nanosecond laser pulse stimulation of spiral ganglion neurons and model cells. Biomed. Opt. Express 5, 1014.

Rhode, W.S. (1971). Observations of the Vibration of the Basilar Membrane in Squirrel Monkeys using the Mössbauer Technique. J. Acoust. Soc. Am. 49, 1218-1231.

Richardson, G.P., Lukashkin, A.N., and Russell, I.J. (2008). The tectorial membrane: one slice of a complex cochlear sandwich: Curr. Opin. Otolaryngol. Head Neck Surg. 16, 458-464.

Richardson, G.P., de Monvel, J.B., and Petit, C. (2011). How the genetics of deafness illuminates auditory physiology. Annu. Rev. Physiol. 73, 311-334.

Richter, C.-P., Emadi, G., Getnick, G., Quesnel, A., and Dallos, P. (2007). Tectorial Membrane Stiffness Gradients. Biophys. J. 93, 2265-2276.

Richter, C.-P., Rajguru, S.M., Matic, A.I., Moreno, E.L., Fishman, A.J., Robinson, A.M., Suh, E., and Walsh, J.T. (2011). Spread of cochlear excitation during stimulation with pulsed infrared radiation: inferior colliculus measurements. J. Neural Eng. 8, 056006.

Rubinstein, J.T. (1991). Analytical theory for extracellular electrical stimulation of nerve with focal electrodes. II. Passive myelinated axon. Biophys. J. 60, 538-555.

Rubinstein, J.T. (1995). Threshold fluctuations in an N sodium channel model of the node of Ranvier. Biophys. J. 68, 779-785.

Rubinstein, J.T., Wilson, B.S., Finley, C.C., and Abbas, P.J. (1999). Pseudospontaneous activity: stochastic independence of auditory nerve fibers with electrical stimulation. Hear. Res. 127, 108-118. 
Ruel, J., Emery, S., Nouvian, R., Bersot, T., Amilhon, B., Van Rybroek, J.M., Rebillard, G., Lenoir, M., Eybalin, M., Delprat, B., et al. (2008). Impairment of SLC17A8 Encoding Vesicular Glutamate Transporter-3, VGLUT3, Underlies Nonsyndromic Deafness DFNA25 and Inner Hair Cell Dysfunction in Null Mice. Am. J. Hum. Genet. 83, 278-292.

Rutherford, M.A., Chapochnikov, N.M., and Moser, T. (2012). Spike Encoding of Neurotransmitter Release Timing by Spiral Ganglion Neurons of the Cochlea. J. Neurosci. 32, 4773-4789.

Sacheli, R., Delacroix, L., Vandenackerveken, P., Nguyen, L., and Malgrange, B. (2013). Gene transfer in inner ear cells: a challenging race. Gene Ther. 20, 237-247.

Sachs, M.B., and Abbas, P.J. (1974). Rate versus level functions for auditory-nerve fibers in cats: toneburst stimuli. J. Acoust. Soc. Am. 56, 1835-1847.

Sachs, M.B., Winslow, R.L., and Sokolowski, B.H. (1989). A computational model for rate-level functions from cat auditory-nerve fibers. Hear. Res. 41, 61-69.

Sahel, J.-A., and Roska, B. (2013). Gene therapy for blindness. Annu. Rev. Neurosci. 36, 467-488.

Sakmann, B., and Neher, E. (1995). Single-Channel Recording (Springer US).

Santarelli, R., Del Castillo, I., Cama, E., Scimemi, P., and Starr, A. (2015). Audibility, speech perception and processing of temporal cues in ribbon synaptic disorders due to OTOF mutations. Hear. Res. 330, 200-212.

Sattig, T., Rickert, C., Bamberg, E., Steinhoff, H.-J., and Bamann, C. (2013). Light-induced movement of the transmembrane helix B in channelrhodopsin-2. Angew. Chem. Int. Ed Engl. 52, 9705-9708.

Schaette, R., and McAlpine, D. (2011). Tinnitus with a normal audiogram: physiological evidence for hidden hearing loss and computational model. J. Neurosci. Off. J. Soc. Neurosci. 31, 13452-13457.

Schild, L.C., and Glauser, D.A. (2015). Dual Color Neural Activation and Behavior Control with Chrimson and CoChR in Caenorhabditis elegans. Genetics 200, 1029-1034.

Schmitz, F. (2009). The Making of Synaptic Ribbons: How They Are Built and What They Do. The Neuroscientist 15, 611-624.

Schneider, F., Gradmann, D., and Hegemann, P. (2013). Ion Selectivity and Competition in Channelrhodopsins. Biophys. J. 105, 91-100.

Schneider, F., Grimm, C., and Hegemann, P. (2015). Biophysics of Channelrhodopsin. Annu. Rev. Biophys. 44, 167-186.

Schoenenberger, P., Grunditz, Å., Rose, T., and Oertner, T.G. (2008). Optimizing the spatial resolution of Channelrhodopsin-2 activation. Brain Cell Biol. 36, 119-127.

Schrauwen, I., Helfmann, S., Inagaki, A., Predoehl, F., Tabatabaiefar, M.A., Picher, M.M., Sommen, M., Seco, C.Z., Oostrik, J., Kremer, H., et al. (2012). A Mutation in CABP2, Expressed in Cochlear Hair Cells, Causes Autosomal-Recessive Hearing Impairment. Am. J. Hum. Genet. 91, 636-645.

Schultz, M., Baumhoff, P., Maier, H., Teudt, I.U., Krüger, A., Lenarz, T., and Kral, A. (2012). Nanosecond laser pulse stimulation of the inner ear-a wavelength study. Biomed. Opt. Express 3, 33323345 .

Schwartz, M.S., and Wilkinson, E.P. (2017). Auditory brainstem implant program development. The Laryngoscope 127, 1909-1915. 
Seal, R.P., Akil, O., Yi, E., Weber, C.M., Grant, L., Yoo, J., Clause, A., Kandler, K., Noebels, J.L., Glowatzki, E., et al. (2008). Sensorineural Deafness and Seizures in Mice Lacking Vesicular Glutamate Transporter 3. Neuron 57, 263-275.

Sengupta, A., Chaffiol, A., Macé, E., Caplette, R., Desrosiers, M., Lampič, M., Forster, V., Marre, O., Lin, J.Y., Sahel, J.-A., et al. (2016). Red-shifted channelrhodopsin stimulation restores light responses in blind mice, macaque retina, and human retina. EMBO Mol. Med. 8, 1248-1264.

Senn, P., Roccio, M., Hahnewald, S., Frick, C., Kwiatkowska, M., Ishikawa, M., Bako, P., Li, H., Edin, F., Liu, W., et al. (2017). NANOCI-Nanotechnology Based Cochlear Implant With Gapless Interface to Auditory Neurons. Otol. Neurotol. 38, e224-e231.

Sergeyenko, Y., Lall, K., Liberman, M.C., and Kujawa, S.G. (2013). Age-related cochlear synaptopathy: an early-onset contributor to auditory functional decline. J. Neurosci. Off. J. Soc. Neurosci. 33, 1368613694.

Sha, S.-H., Kanicki, A., Dootz, G., Talaska, A.E., Halsey, K., Dolan, D., Altschuler, R., and Schacht, J. (2008). Age-related auditory pathology in the CBA/J mouse. Hear. Res. 243, 87-94.

Shapiro, M.G., Homma, K., Villarreal, S., Richter, C.-P., and Bezanilla, F. (2012). Infrared light excites cells by changing their electrical capacitance. Nat. Commun. 3, 736.

Sharma, A., Dorman, M.F., and Spahr, A.J. (2002). Rapid development of cortical auditory evoked potentials after early cochlear implantation. Neuroreport 13,1365-1368.

Shearer, A.E., Black-Ziegelbein, E.A., Hildebrand, M.S., Eppsteiner, R.W., Ravi, H., Joshi, S., Guiffre, A.C., Sloan, C.M., Happe, S., Howard, S.D., et al. (2013). Advancing Genetic Testing for Deafness with Genomic Technology. J. Med. Genet. 50.

Shepherd, R.K., and Javel, E. (1997). Electrical stimulation of the auditory nerve. I. Correlation of physiological responses with cochlear status. Hear. Res. 108, 112-144.

Shevchenko, V., Mager, T., Kovalev, K., Polovinkin, V., Alekseev, A., Juettner, J., Chizhov, I., Bamann, C., Vavourakis, C., Ghai, R., et al. (2017). Inward H+ pump xenorhodopsin: Mechanism and alternative optogenetic approach. Sci. Adv. 3.

Shimano, T., Fyk-Kolodziej, B., Mirza, N., Asako, M., Tomoda, K., Bledsoe, S., Pan, Z.H., Molitor, S., and Holt, A.G. (2013). Assessment of the AAV-mediated expression of channelrhodopsin-2 and halorhodopsin in brainstem neurons mediating auditory signaling. Brain Res. 1511, 138-152.

Shnerson, A., and Pujol, R. (1981). Age-related changes in the C57BL/6J mouse cochlea. I. Physiological findings. Dev. Brain Res. 2, 65-75.

Shrestha, B.R., Chia, C., Wu, L., Kujawa, S.G., Liberman, M.C., and Goodrich, L.V. (2018). Sensory Neuron Diversity in the Inner Ear Is Shaped by Activity. Cell 174, 1229-1246.e17.

Siegel, J.H. (1992). Spontaneous synaptic potentials from afferent terminals in the guinea pig cochlea. Hear. Res. 59, 85-92.

Simunovic, M.P., Shen, W., Lin, J.Y., Protti, D.A., Lisowski, L., and Gillies, M.C. (2018). Optogenetic approaches to vision restoration. Exp. Eye Res. 178, 15-26.

Sineshchekov, O.A., Jung, K.H., and Spudich, J.L. (2002). Two rhodopsins mediate phototaxis to lowand high-intensity light in Chlamydomonasreinhardtii. Proc. Natl. Acad. Sci. U. S. A. 99, 8689.

Skinner, M.W., Clark, G.M., Whitford, L.A., Seligman, P.M., Staller, S.J., Shipp, D.B., Shallop, J.K., Everingham, C., Menapace, C.M., and Arndt, P.L. (1994). Evaluation of a new spectral peak coding strategy for the Nucleus 22 Channel Cochlear Implant System. Am. J. Otol. 15 Suppl 2, 15-27. 
Smith, R.H. (2008). Adeno-associated virus integration: virus versus vector. Gene Ther. 15, 817-822.

Smith, K.E., Browne, L., Selwood, D.L., McAlpine, D., and Jagger, D.J. (2015). Phosphoinositide Modulation of Heteromeric Kv1 Channels Adjusts Output of Spiral Ganglion Neurons from Hearing Mice. J. Neurosci. 35, 11221-11232.

Smith, R.J.H., Jr, J.F.B., and White, K.R. (2005). Sensorineural hearing loss in children. 365, 12.

Smith, Z.M., Delgutte, B., and Oxenham, A.J. (2002). Chimaeric sounds reveal dichotomies in auditory perception. Nature 416, 87-90.

Spahr, A.J., Dorman, M.F., and Loiselle, L.H. (2007). Performance of patients using different cochlear implant systems: effects of input dynamic range. Ear Hear. 28, 260-275.

Stamataki, S., Francis, H.W., Lehar, M., May, B.J., and Ryugo, D.K. (2006). Synaptic alterations at inner hair cells precede spiral ganglion cell loss in aging C57BL/6J mice. Hear. Res. 221, 104-118.

Stockklausner, C., Ludwig, J., Ruppersberg, J.P., and Klöcker, N. (2001). A sequence motif responsible for ER export and surface expression of Kir2.0 inward rectifier K(+) channels. FEBS Lett. 493, 129133.

Strenzke, N., Chanda, S., Kopp-Scheinpflug, C., Khimich, D., Reim, K., Bulankina, A.V., Neef, A., Wolf, F., Brose, N., Xu-Friedman, M.A., et al. (2009). Complexin-I Is Required for High-Fidelity Transmission at the Endbulb of Held Auditory Synapse. J. Neurosci. 29, 7991-8004.

Subramaniam, S., Gerstein, M., Oesterhelt, D., and Henderson, R. (1993). Electron diffraction analysis of structural changes in the photocycle of bacteriorhodopsin. EMBO J. 12, 1-8.

Suzuki, J., Hashimoto, K., Xiao, R., Vandenberghe, L.H., and Liberman, M.C. (2017). Cochlear gene therapy with ancestral AAV in adult mice: complete transduction of inner hair cells without cochlear dysfunction. Sci. Rep. 7, 45524.

Taberner, A.M., and Liberman, M.C. (2005). Response Properties of Single Auditory Nerve Fibers in the Mouse. J. Neurophysiol. 93, 557-569.

Tasaki, I. (1954). Nerve impulses in individual auditory nerve fibers of guinea pig. J. Neurophysiol. 17, 97-122.

Temchin, A.N., and Ruggero, M.A. (2010). Phase-Locked Responses to Tones of Chinchilla Auditory Nerve Fibers: Implications for Apical Cochlear Mechanics. JARO J. Assoc. Res. Otolaryngol. 11, 297318.

Temchin, A.N., Rich, N.C., and Ruggero, M.A. (2008). Threshold Tuning Curves of Chinchilla Auditory-Nerve Fibers. I. Dependence on Characteristic Frequency and Relation to the Magnitudes of Cochlear Vibrations. J. Neurophysiol. 100, 2889-2898.

Teudt, I.U., Maier, H., Richter, C.-P., and Kral, A. (2011). Acoustic events and "optophonic" cochlear responses induced by pulsed near-infrared laser. IEEE Trans. Biomed. Eng. 58, 1648-1655.

Thompson, A.C., Fallon, J.B., Wise, A.K., Wade, S.A., Shepherd, R.K., and Stoddart, P.R. (2015). Infrared neural stimulation fails to evoke neural activity in the deaf guinea pig cochlea. Hear. Res. 324, $46-53$.

Töpperwien, M., Gradl, R., Keppeler, D., Vassholz, M., Meyer, A., Hessler, R., Achterhold, K., Gleich, B., Dierolf, M., Pfeiffer, F., et al. (2018). Propagation-based phase-contrast x-ray tomography of cochlea using a compact synchrotron source. Sci. Rep. 8, 4922. 
Toth, T., Deak, L., Fazakas, F., Zheng, J., Muszbek, L., and Sziklai, I. (2007). A new mutation in the human pres gene and its effect on prestin function. Int. J. Mol. Med. 20, 545-550.

Towne, C., Pertin, M., Beggah, A.T., Aebischer, P., and Decosterd, I. (2009). Recombinant adenoassociated virus serotype 6 (rAAV2/6)-mediated gene transfer to nociceptive neurons through different routes of delivery. Mol. Pain 5, 52.

Ugalde, J.A., Podell, S., Narasingarao, P., and Allen, E.E. (2011). Xenorhodopsins, an enigmatic new class of microbial rhodopsins horizontally transferred between archaea and bacteria. Biol. Direct 6, 52 .

Varga, R., Avenarius, M.R., Kelley, P.M., Keats, B.J., Berlin, C.I., Hood, L.J., Morlet, T.G., Brashears, S.M., Starr, A., Cohn, E.S., et al. (2006). OTOF mutations revealed by genetic analysis of hearing loss families including a potential temperature sensitive auditory neuropathy allele. J. Med. Genet. 43, 576581.

Verma, R.U., Guex, A.A., Hancock, K.E., Durakovic, N., McKay, C.M., Slama, M.C.C., Brown, M.C., and Lee, D.J. (2014). Auditory responses to electric and infrared neural stimulation of the rat cochlear nucleus. Hear. Res. 310, 69-75.

Versnel, H., Prijs, V.F., and Schoonhoven, R. (1997). Auditory-nerve fiber responses to clicks in guinea pigs with a damaged cochlea. J. Acoust. Soc. Am. 101, 993-1009.

Versteegh, C.P.C., Meenderink, S.W.F., and van der Heijden, M. (2011). Response Characteristics in the Apex of the Gerbil Cochlea Studied Through Auditory Nerve Recordings. JARO J. Assoc. Res. Otolaryngol. 12, 301-316.

Viemeister, N.F. (1983). Auditory intensity discrimination at high frequencies in the presence of noise. Science 221, 1206-1208.

Viemeister, N.F. (1988). Intensity coding and the dynamic range problem. Hear. Res. 34, 267-274.

Viemeister, N.F., and Bacon, S.P. (1988). Intensity discrimination, increment detection, and magnitude estimation for 1-kHz tones. J. Acoust. Soc. Am. 84, 172-178.

Vierock, J., Grimm, C., Nitzan, N., and Hegemann, P. (2017). Molecular determinants of proton selectivity and gating in the red-light activated channelrhodopsin Chrimson. Sci. Rep. 7, 9928.

Vonck, J. (2000). Structure of the bacteriorhodopsin mutant F219L N intermediate revealed by electron crystallography. EMBO J. 19, 2152-2160.

Wang, Y., Hirose, K., and Liberman, M.C. (2002). Dynamics of Noise-Induced Cellular Injury and Repair in the Mouse Cochlea. J. Assoc. Res. Otolaryngol. 3, 248-268.

Wang, Y., Chang, Q., Tang, W., Sun, Y., Zhou, B., Li, H., and Lin, X. (2009). Targeted connexin26 ablation arrests postnatal development of the organ of Corti. Biochem. Biophys. Res. Commun. 385, 3337.

Wangemann, P., and Schacht, J. (1996). Homeostatic Mechanisms in the Cochlea. In The Cochlea, P. Dallos, A.N. Popper, and R.R. Fay, eds. (Springer New York), pp. 130-185.

Wassmer, S.J., Carvalho, L.S., György, B., Vandenberghe, L.H., and Maguire, C.A. (2017). Exosomeassociated AAV2 vector mediates robust gene delivery into the murine retina upon intravitreal injection. Sci. Rep. 7, 45329.

Watkins, P.V., and Barbour, D.L. (2011). Rate-level responses in awake marmoset auditory cortex. Hear. Res. 275, 30-42. 
Weiss, R.S., Voss, A., and Hemmert, W. (2016). Optogenetic stimulation of the cochlea-A review of mechanisms, measurements, and first models. Netw. Comput. Neural Syst. 27, 212-236.

Wells, J., Konrad, P., Kao, C., Jansen, E.D., and Mahadevan-Jansen, A. (2007). Pulsed laser versus electrical energy for peripheral nerve stimulation. J. Neurosci. Methods 163, 326-337.

Wen, B., Wang, G.I., Dean, I., and Delgutte, B. (2009). Dynamic Range Adaptation to Sound Level Statistics in the Auditory Nerve. J. Neurosci. 29, 13797-13808.

WHO (2018). Deafness and hearing loss.

Wichmann, C. (2015). Molecularly and structurally distinct synapses mediate reliable encoding and processing of auditory information. Hear. Res. 330, 178-190.

Wichmann, C., and Moser, T. (2015). Relating structure and function of inner hair cell ribbon synapses. Cell Tissue Res.

Wilson, B.S. (2018). The cochlear implant and possibilities for narrowing the remaining gaps between prosthetic and normal hearing. World J. Otorhinolaryngol. - Head Neck Surg. 3, 200-210.

Wilson, B.S., and Dorman, M.F. (2008). Cochlear implants: current designs and future possibilities. J. Rehabil. Res. Dev. 45, 695-730.

Wilson, B.S., Finley, C.C., Farmer, J.C., Lawson, D.T., Weber, B.A., Wolford, R.D., Kenan, P.D., White, M.W., Merzenich, M.M., and Schindler, R.A. (1988). Comparative studies of speech processing strategies for cochlear implants. The Laryngoscope 98, 1069-1077.

Wilson, B.S., Finley, C.C., Lawson, D.T., Wolford, R.D., Eddington, D.K., and Rabinowitz, W.M. (1991). Better speech recognition with cochlear implants. Nature 352, 236-238.

Winter, I.M., Robertson, D., and Yates, G.K. (1990). Diversity of characteristic frequency rate-intensity functions in guinea pig auditory nerve fibres. Hear. Res. 45, 191-202.

Wrobel, C., Dieter, A., Huet, A., Keppeler, D., Duque-Afonso, C.J., Vogl, C., Hoch, G., Jeschke, M., and Moser, T. (2018). Optogenetic stimulation of cochlear neurons activates the auditory pathway and restores auditory-driven behavior in deaf adult gerbils. Sci. Transl. Med. 10, eaao0540.

Wu, J., Seregard, S., and Algvere, P.V. (2006). Photochemical Damage of the Retina. Surv. Ophthalmol. $51,461-481$.

Xu, R., Janson, C.G., Mastakov, M., Lawlor, P., Young, D., Mouravlev, A., Fitzsimons, H., Choi, K.L., Ma, H., Dragunow, M., et al. (2001). Quantitative comparison of expression with adeno-associated virus (AAV-2) brain-specific gene cassettes. Gene Ther. 8, 1323-1332.

Yang, C.-H., Schrepfer, T., and Schacht, J. (2015). Age-related hearing impairment and the triad of acquired hearing loss. Front. Cell. Neurosci. 9, 276.

Yasunaga, S., Grati, M., Cohen-Salmon, M., El-Amraoui, A., Mustapha, M., Salem, N., El-Zir, E., Loiselet, J., and Petit, C. (1999). A mutation in OTOF, encoding otoferlin, a FER-1-like protein, causes DFNB9, a nonsyndromic form of deafness. Nat. Genet. 21, 363-369.

Yates, G.K., Winter, I.M., and Robertson, D. (1990). Basilar membrane nonlinearity determines auditory nerve rate-intensity functions and cochlear dynamic range. Hear. Res. 45, 203-219.

Yin, Y., Liberman, L.D., Maison, S.F., and Liberman, M.C. (2014). Olivocochlear innervation maintains the normal modiolar-pillar and habenular-cuticular gradients in cochlear synaptic morphology. J. Assoc. Res. Otolaryngol. JARO 15, 571-583. 
Yizhar, O., Fenno, L.E., Davidson, T.J., Mogri, M., and Deisseroth, K. (2011). Optogenetics in neural systems. Neuron 71, 9-34.

Youm, I., and Li, W. (2018). Cochlear hair cell regeneration: an emerging opportunity to cure noiseinduced sensorineural hearing loss. Drug Discov. Today 23, 1564-1569.

Yu-Wai-Man, P., Griffiths, P.G., Gorman, G.S., Lourenco, C.M., Wright, A.F., Auer-Grumbach, M., Toscano, A., Musumeci, O., Valentino, M.L., Caporali, L., et al. (2010). Multi-system neurological disease is common in patients with OPA1 mutations. Brain J. Neurol. 133, 771-786.

Zeng, F.G. (2017). Challenges in Improving Cochlear Implant Performance and Accessibility. IEEE Trans. Biomed. Eng. 64, 1662-1664.

Zeng, F.-G., and Galvin, J.J.I. (1999). Amplitude Mapping and Phoneme Recognition in Cochlear Implant Listeners. Ear Hear. 20, 60-74.

Zeng, F.-G., Rebscher, S., Harrison, W.V., Sun, X., and Feng, H. (2008). Cochlear Implants:System Design, Integration and Evaluation. IEEE Rev. Biomed. Eng. 1, 115-142.

Zhang, F., Wang, L.-P., Brauner, M., Liewald, J.F., Kay, K., Watzke, N., Wood, P.G., Bamberg, E., Nagel, G., Gottschalk, A., et al. (2007a). Multimodal fast optical interrogation of neural circuitry. Nature 446, 633-639.

Zhang, F., Miller, C.A., Robinson, B.K., Abbas, P.J., and Hu, N. (2007b). Changes Across Time in Spike Rate and Spike Amplitude of Auditory Nerve Fibers Stimulated by Electric Pulse Trains. JARO J. Assoc. Res. Otolaryngol. 8, 356-372.

Zhang, F., Prigge, M., Beyrière, F., Tsunoda, S.P., Mattis, J., Yizhar, O., Hegemann, P., and Deisseroth, K. (2008). Red-shifted optogenetic excitation: a tool for fast neural control derived from Volvox carteri. Nat. Neurosci. 11, 631-633.

Zhang, W., Kim, S.M., Wang, W., Cai, C., Feng, Y., Kong, W., and Lin, X. (2018). Cochlear Gene Therapy for Sensorineural Hearing Loss: Current Status and Major Remaining Hurdles for Translational Success. Front. Mol. Neurosci. 11.

Zheng, J., Shen, W., He, D.Z.., Long, K.B., Madison, L.D., and Dallos, P. (2000). Prestin is the motor protein of cochlear outer hair cells. Nature 405, 149-155.

Zierhofer, C.M., Hochmair-Desoyer, I.J., and Hochmair, E.S. (1995). Electronic design of a cochlear implant for multichannel high-rate pulsatile stimulation strategies. IEEE Trans. Rehabil. Eng. 3, 112116.

Zinn, E., Pacouret, S., Khaychuk, V., Turunen, H.T., Carvalho, L.S., Andres-Mateos, E., Shah, S., Shelke, R., Maurer, A.C., Plovie, E., et al. (2015). In Silico Reconstruction of the Viral Evolutionary Lineage Yields a Potent Gene Therapy Vector. Cell Rep. 12, 1056-1068.

Zolotukhin, S., Byrne, B.J., Mason, E., Zolotukhin, I., Potter, M., Chesnut, K., Summerford, C., Samulski, R.J., and Muzyczka, N. (1999). Recombinant adeno-associated virus purification using novel methods improves infectious titer and yield. Gene Ther. 6, 973-985. 


\section{Acknowledgements}

First, I would like to thank Prof. Tobias Moser for providing me with the opportunity to work in this project. I appreciate his encouragement, scientific advice and indefatigable efforts to promote our institute and our work. I want to extend my gratitude to Prof. Silvio Rizzoli and Prof. Tim Gollisch, for their time and kindness co-supervising the progress of my project.

I want to thank Nicola Strenzke and Gulnara Yamabaeva for teaching me auditory nerve recordings. Their patience and support helped me gaining confidence at such delicate procedure. I further want to thank Thomas Mager and Prof. Ernst Bamberg for the fluent and fruitful communication during our collaboration.

I am especially grateful to Carlos J. Duque for his priceless support throughout my time here, for our enriching discussions and for his friendship: he is the most creative person I have ever (and will ever) met, and I look up to him for that; to Özge D. Özçete for being always ready to help, listen and discuss: her comradeship was genuine and our "complain coffee breaks" were an essential voodoo exercise for things to work; and to Lina M. Jaime for her help, understanding and critical thinking: she always made me see things from a different point of view, and her serenity put a hold on the everlasting rush of $\mathrm{PhD}$ life. Science needs you all!

Moreover, I want to thank Christian Vogl because all I learnt from him during my Master's was essential for my $\mathrm{PhD}$, and for his advice on immunostainings and imaging. Many thanks to Antoine H. Huet and Christian Wrobel for their help at in vivo experiments, discussion of results; and for their programming skills and collaboration at different stages of the project, together with Alexander Dieter and Gerhard Hoch. I also want to thank Daniel Keppeler and Ricardo Merino for their commitment and collaboration in the course of our shared project.

I thank Vladan Rankovic for his availability for discussion, and together with Sangyong Jung and Christiane Senger-Freitag for performing AAV injections. A big thanks to Christiane, Sandra Gerke and Daniela Gerke, for their superb technical assistance. Moreover, I would like to thank Patricia Räke-Kügler and Susann Müller for dealing with bureaucracy for us, for their availability and for being able to solve the unsolvable. I would also like to thank Steffen Burkhardt and Kerstin Grüniger from the Molecular Biology program for their assistance throughout my PhD. 
This years would have been much harder without the support of my former flatmates: Elina Galani and Leticia Domínguez. I want to thank Leticia very especially for her patience, for her unique ability to understand me and for showing me the value of positive thinking. I would not have enjoyed those "dias nublados" without her company and friendship. I do not want to forget the inestimable support of Blanca Rincón during these last months. She became "mi hija" long ago and I felt honored to overlap and share the thesis-writing time with her.

I profoundly thank my parents and my brother for their infallible support, encouragement and understanding in any kind of matter. Their care, loyalty and diligence are my best example and I will not ever be thankful enough for it. This thesis is as theirs as much as mine.

Finally, and most importantly, I want to thank Lydia Domínguez for her empathy, for which I look up to her; for her never-ending support and love. She always knew how to make my worries shrink and showed me the value of speaking out; and I cherished her efforts at trying to understand this or that experiment and her invaluable care and company throughout these years. «Y de la tierra perdida en la infancia / Al mundo perecedero / Bendecida fue la causa de mi fortuna» 


\section{Appendix}

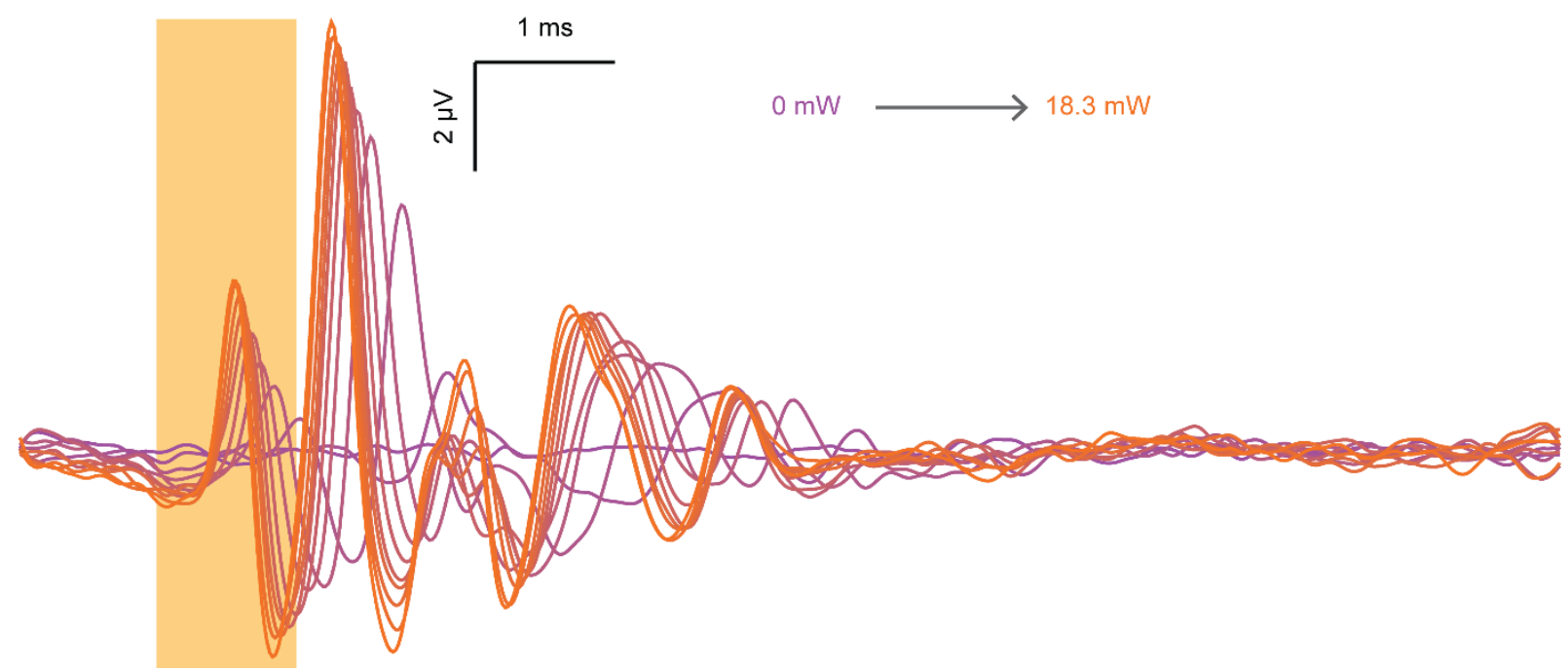

Figure A1: oABR recorded from a C57BL6/J mouse at 51 weeks of age at increasing stimulus intensity $(1 \mathrm{~ms}$ pulse width at $20 \mathrm{~Hz})$. Stimulation is marked by the orange rectangle. 